\title{
Cucurbituril-Based Molecular Recognition
}

Steven J. Barrow, ${ }^{+, \ddagger}$ Setu Kasera, ${ }^{+, \ddagger}$ Matthew J. Rowland, ${ }^{+, \ddagger}$ Jesús del Barrio, ${ }^{+}$ and Oren A. Scherman ${ }^{*}+$

Melville Laboratory for Polymer Synthesis, Department of Chemistry, Lensfield Road, University of Cambridge, Cambridge CB2 1EW

E-mail: oas23@cam.ac.uk

Phone: +44 (0)1223 334370

\section{Contents}

1 Introduction

2 Thermodynamics of $\mathrm{CB}[n]$-guest binding

3 Specific binding properties of $\mathrm{CB}[5]$

4 Specific binding properties of $\mathrm{CB}[6]$

$5 \quad$ Specific binding properties of $\mathrm{CB}[7]$

6 Specific binding properties of $\mathrm{CB}[8]$

7 Encapsulation of gaseous species in $\mathrm{CB}[n]$

$7.1 \mathrm{CB}[5]$ gas sorption . . . . . . . . . . . . . . . . . . . . . . . . . . . . 29

\footnotetext{
*To whom correspondence should be addressed

${ }^{+}$University of Cambridge

$\ddagger$ Contributed equally to this work
} 
$7.2 \mathrm{CB}[6]$ gas sorption $\ldots \ldots \ldots \ldots \ldots \ldots \ldots \ldots \ldots \ldots \ldots \ldots \ldots$

7.3 Gas sorption in higher $\mathrm{CB}[n]$ homologues . . . . . . . . . . . . . . 34

8 Cucurbit $[n]$ urils on surfaces $\quad 35$

8.1 Electrostatic interactions . . . . . . . . . . . . . . . . . 35

8.2 Guest-mediated interactions . . . . . . . . . . . . . . . . 45

8.3 Covalent immobilization . . . . . . . . . . . . . . . . . . 50

8.4 Characterization of $\mathrm{CB}[n]$ host-guest binding on surfaces $\ldots \ldots \ldots 52$

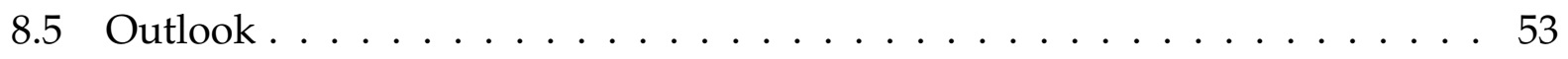

9 Supramolecular Polymers $\quad 54$

9.1 Small molecule based supramolecular polymers . . . . . . . . . . . . 54

$9.2 \mathrm{CB}[8]$ mediated supramolecular block copolymerization $\ldots \ldots$. . . . . 65

9.2.1 Supramolecular assembly of macromolecules . . . . . . . . . . 65

9.2 .2 Conclusion and outlook . . . . . . . . . . . . . . 70

$\begin{array}{ll}10 \text { Molecular recognition in 3D supramolecular networks } & 70\end{array}$

$10.1 \mathrm{CB}[8]$ complexes crosslink guest functional polymers $\ldots \ldots \ldots 71$

10.2 Dynamics of crosslinking influences material properties . . . . . . . . . 76

10.3 Composite soft materials utilizing $\mathrm{CB}[8]$ host-guest interactions $\ldots . .7 .78$

10.4 From monohydroxylated-CB $[n]$ to functional materials $\ldots \ldots$. . . . . 80

10.5 Microcapsule formation through $\mathrm{CB}[8]$ ternary complexation $\ldots \ldots \ldots 2$

$10.6 \mathrm{CB}[n]$ crosslinked polymer networks outlook . . . . . . . . . . . . 85

11 Catalysis within Cucurbituril Cavities $\quad 86$

$11.1[3+2]$-cycloadditions catalysed by CB[6] . . . . . . . . . . . . 86

$11.2 \mathrm{CB}[n]$ catalysed solvolysis reactions $\ldots \ldots \ldots \ldots$. . . . . . . 87

$11.3 \mathrm{CB}[n]$ catalyzed oxidation reactions . . . . . . . . . . . . . . . . . 89

11.4 Photocatalysis . . . . . . . . . . . . . . . . . . . . . 89 
11.5 Metal cation assisted $\mathrm{CB}[n]$ catalysis $\ldots \ldots \ldots$. . . . . . . . . 93

11.6 Reaction inhibition by cucurbit[n]urils . . . . . . . . . . . . . . . 96

$\begin{array}{ll}12 \text { Recognition by } \mathrm{CB}[n] \text { in biological systems } & 100\end{array}$

12.1 Amino acid and short peptide recognition by $\mathrm{CB}[n] \ldots \ldots$

12.2 Non-covalent protein conjugation . . . . . . . . . . . . . . . . . . . 104

12.3 Further application to biochemistry $\ldots \ldots \ldots$. . . . . . . . . 106

12.4 Profiling toxicity of cucurbit $[n]$ uril $\ldots \ldots \ldots \ldots$

12.5 Outlook . . . . . . . . . . . . . . . . . . . . . . 110

$\begin{array}{ll}13 \text { Summary and outlook } & 111\end{array}$

$\begin{array}{ll}14 \text { Tables } & 113\end{array}$

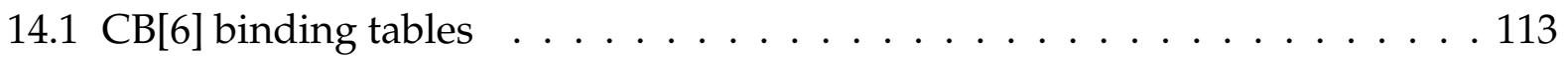

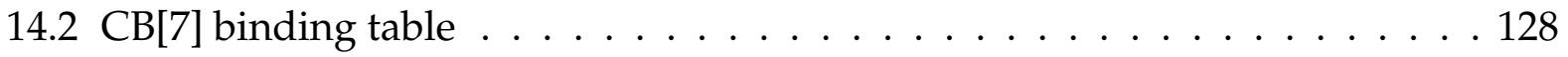

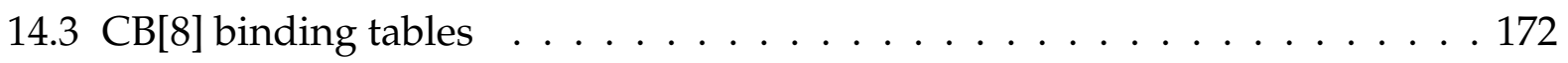

$\begin{array}{ll}15 \text { Biographies } & 206\end{array}$

\section{Introduction}

The product of the condensation reaction between glycoluril and formaldehyde was first reported in a thesis by Eberhard Meyer in 1904, ${ }^{1}$ then published by Behrend et al. in $1905,{ }^{2}$ however, it wasn't until 1981 that a product was successfully crystallized from the reaction: a macrocycle consisting of 6 glycoluril units bound together by 12 methylene bridges (see Figure 1). ${ }^{3}$ The macrocycle was named cucurbituril, given its resemblance to a pumpkin (which belongs to the cucurbitaceae family), yet often abbreviated as CB[6], highlighting the 6 glycoluril building blocks that constitute the macrocycle.

During the 1980s and 1990s, interest in crystal engineering and non-covalent interac- 

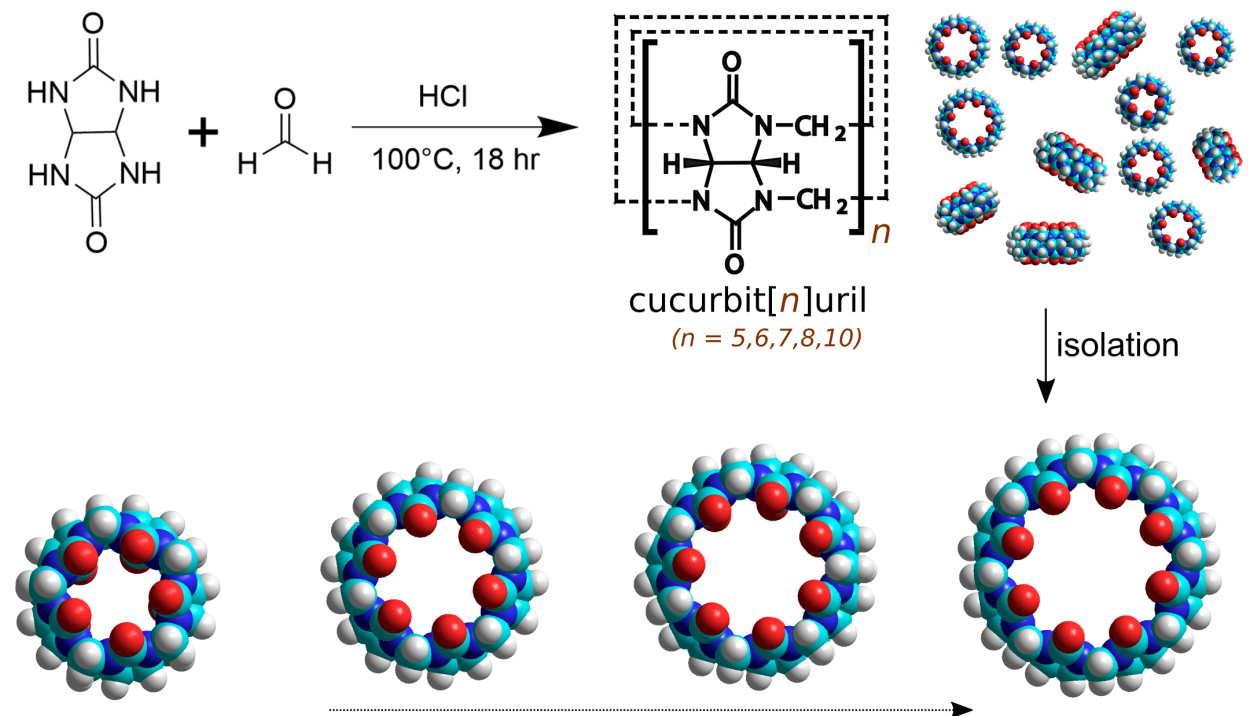

increasing cavity size

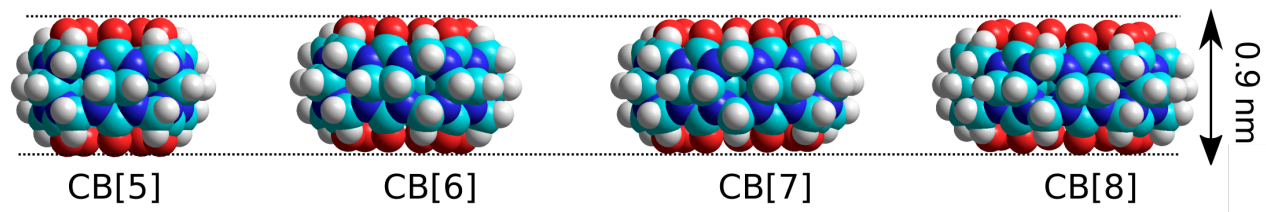

Figure 1: Top: The reaction between glycoluril and formaldehyde. Bottom: space-filling models of CB[5]$\mathrm{CB}[8]$ demonstrating the increasing size, yet constant height, of the $\mathrm{CB}[n]$ macrocycles.

tions saw the popularity of cucurbituril increase due to its ability to facilitate non-covalent binding. This is made possible via the formation of complexes with cations through interactions with the carbonyl lined portals of $\mathrm{CB}[6]$, along with its ability to internalize alkyl chains within its hydrophobic cavity. Between 2000 and 2002, Kim ${ }^{4}$ and Day ${ }^{5,6}$ modified the initial reactions conditions of Meyer and Behrend, to synthesize a variety of glycoluril based cucurbituril macrocycles. The new additions to the cucurbituril family were named cucurbit[5]uril, cucurbit[7]uril, cucurbit[8]uril and cucurbit[10]uril in light of them containing $5,7,8$ or 10 glycoluril units respectively. These discoveries enhanced the applicability of cucurbiturils, as the bigger cavity size of the larger CBs enables them to form 1:1 binary complexes $(\mathrm{CB}[7])$, and even 1:1:1 heteroternary complexes (CB[8], see Figure 2), with aromatic compounds. A variety of $\mathrm{CB}[n]$ homologues have been discovered since the work of Kim and Day, including functionalized $\mathrm{CB}[n],{ }^{7-9}$ nor-seco-CB $[10],{ }^{10}$ inverted $\mathrm{CB}[n]^{11}$ and many others. 


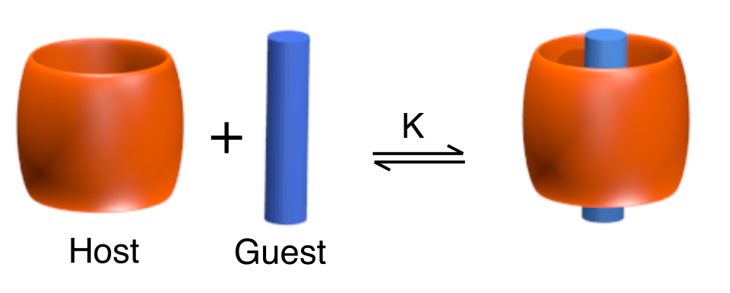

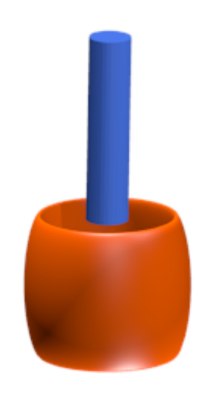

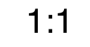
exclusion

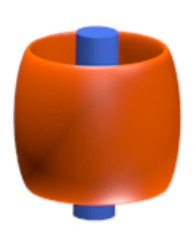

$1: 1$

inclusion

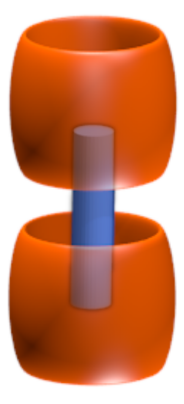

$2: 1$

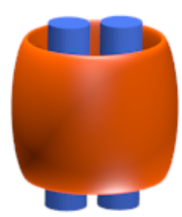

$1: 2$ homodimer

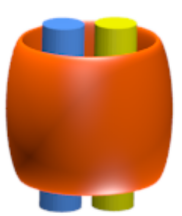

$1: 1: 1$ heteroternary

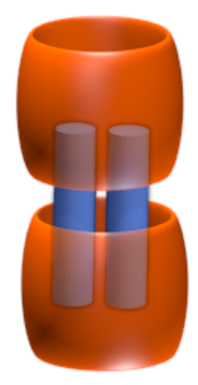

$2: 2$

Figure 2: $\mathrm{CB}[n]$ inclusion and exclusion complexes.

$\mathrm{CB}[5]-\mathrm{CB}[8]$ have found a variety of uses given their ability to form binary and ternary host-guest complexes, and have therefore impacted a wide variety of scientific research areas. Several previous reviews have specifically highlighted the synthesis, functionalization, host-guest binding properties of $\mathrm{CB}[n]^{12-17}$ and their applications in drug delivery, ${ }^{18}$ fluorescence spectroscopy, ${ }^{19}$ catalysis ${ }^{20}$ and nanotechnology. ${ }^{21,22}$ More recently, the contribution of $\mathrm{CB}[n]$ in self-assembly and engineering of nanomaterials has also received attention, ${ }^{23}$ where the role of $\mathrm{CB}[n]$ as a capping agent to stabilize metallic nanoparticles has been emphasized. The diversity of these reviews are evidence of the far-reaching impact cucurbit[ $n]$ urils have had in just the past three decades.

With the vast majority of applications of CB relying on its versatility in forming dynamic complexes with a variety of chemical species, research efforts by the cucurbit $[n]$ uril community continue to be directed towards identifying and characterizing suitable guest molecules. Indeed, designing functional systems containing $\mathrm{CB}[n]$ would not be possible without the knowledge of binding strengths and the kinetics of complexation and de-complexation of the host molecule with the guests of interest. While the interactions 
of the macrocyclic host family have been reported widely, the characterization techniques and conditions under which these are reported have not been comprehensively reviewed. Moreover, the plethora of $\mathrm{CB}[n]$ guest species and their binding constants have not been tabulated and made available in one place. The present review highlights the latest developments in the understanding and applications of the molecular recognition properties of $\mathrm{CB}[n]$, with particular emphasis on materials design.

In this review, we cover the vast areas of scientific research upon which $\mathrm{CB}[n] \mathrm{s}$ have impacted, with a focus on the guest recognition properties of $\mathrm{CB}[\mathrm{n}]$, largely but not wholly concerning of their utilisation in solution phase where most applications have arisen. To understand the binding properties of $\mathrm{CB}[\mathrm{n}]$, a general overview of the thermodynamics of $\mathrm{CB}[n]$ binding is first discussed, followed by an individual analysis of the specific binding properties of $\mathrm{CB}[5]-\mathrm{CB}[8]$. Looking towards the application of $\mathrm{CB}[\mathrm{n}]$ complexation, the areas of gas encapsulation, $\mathrm{CB}[\mathrm{n}] \mathrm{s}$ on surfaces, $\mathrm{CB}[\mathrm{n}]$ mediated supramolecular polymers, Molecular recognition in 3D supramolecular networks, $\mathrm{CB}[\mathrm{n}]$ catalysis and reaction templating, and $\mathrm{CB}[\mathrm{n}]$ recognition in biological systems will each be discussed in turn.

Additionally, this review provides the reader with a comprehensive guide to the guest molecules that have been studied with $\mathrm{CB}[5,6,7,8]$. The tables, present at the end of this review, cover first guests that have been demonstrated to bind with cucurbiturils, and include the binding constants $(\log K)$ for each interaction, along with the conditions in which the binding parameters have been reported. For the case of $\mathrm{CB}[8]$, tables covering the binding constants for 1:1, 1:2 and 1:1:1 complexes have been included (see Figure 2). The binding constants, as well as the conditions under which the binding constants are measured, have been emphasized with the hope that this will encourage the development of new applications for known guest molecules, as well as lead to the realization of new guest species for $\mathrm{CB}[n]$ host-guest complexes. 


\section{Thermodynamics of $\mathrm{CB}[n]$-guest binding}

Cucurbit $[n]$ urils consist of $n$ glycoluril molecules that are bound in a ring-like arrangement via methylene bridges. This arrangement of the glycoluril sub-units is key to understanding the binding and encapsulation properties of CBs, as the number of repeat units defines the portal size and cavity volume, and the alignment of the glycoluril units results in a hydrophobic cavity with carbonyl-lined portals. Understanding these aspects of $\mathrm{CB}[\mathrm{n}]$ macrocycles can enable researchers to design guest species for high affinity binding, tailored towards specific applications. Each of these aspects will now be considered.

The dipolar nature of the carbonyl-fringed portals of $\mathrm{CB}[n] \mathrm{s}$ make the portals highly attractive for cation binding through the ion-dipole effect. Figure 3 shows the electrostatic potential for $\mathrm{CB}[52]-\mathrm{CB}[8]$, which highlights the strength of the negative charge associated with the portals of $\mathrm{CB}$. Indeed, the first reports of $\mathrm{CB}[n]$ binding involved metal cations acting as 'lids' bound to the portals of CB. ${ }^{24}$ Alkali metals, ${ }^{24,25}$ alkaline earth metals, ${ }^{26-28}$ transition metals, ${ }^{28-30}$ lanthanides and actinides, ${ }^{28,31-33}$ as well as ammonium and imidazolium ions ${ }^{34}$ have been shown to bind to $\mathrm{CB}$ portals, and are often required to enhance the solubility of the less water soluble CBs. The portals have also been suggested to provide a constrictive steric barrier towards binding, enhancing the persistence of $\mathrm{CB}[n]$ complexes. ${ }^{35}$ The coordination chemistry of $\mathrm{CB}[n] \mathrm{s}$ has been recently reviewed, ${ }^{36}$ and thus will not be discussed any further here.

Whilst the portals of $\mathrm{CB}[n] \mathrm{s}$ are highly electronegative, the cavities of the macrocycle are encompassed by the fused rings of the glycoluril subunits, which leave no functional groups or electron pairs accessible to the inner cavity. Thus, the inner cavity of $\mathrm{CB}[n] \mathrm{s}$ are remarkably hydrophobic, and show a preference towards the encapsulation of hydrophobic compounds. Hence, alkylated ammonium and imidazolium ions bind to $\mathrm{CB}[n] \mathrm{s}$ such that the cation remains at the portal region, whilst the alkyl chains become encapsulated within the $\mathrm{CB}$ cavity given its inherent hydrophobicity. ${ }^{37,38}$ It should not be surprising then to discover that compounds with high binding constants towards $\mathrm{CB}[n] \mathrm{s}$ 
a)

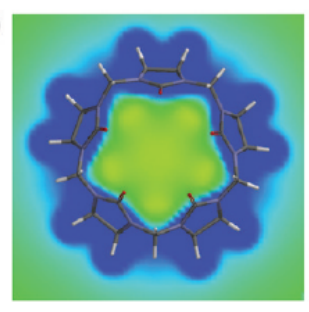

b)

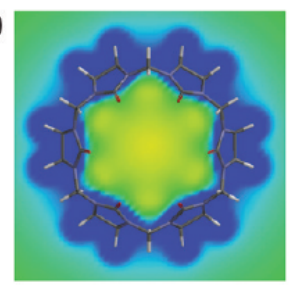

c)

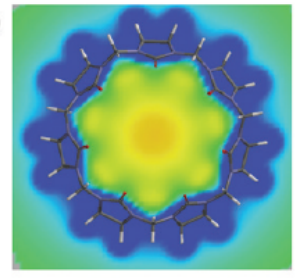

d)

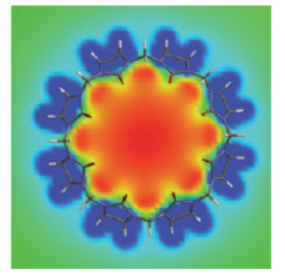

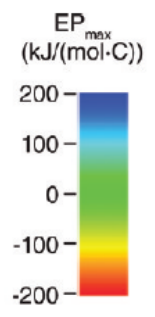
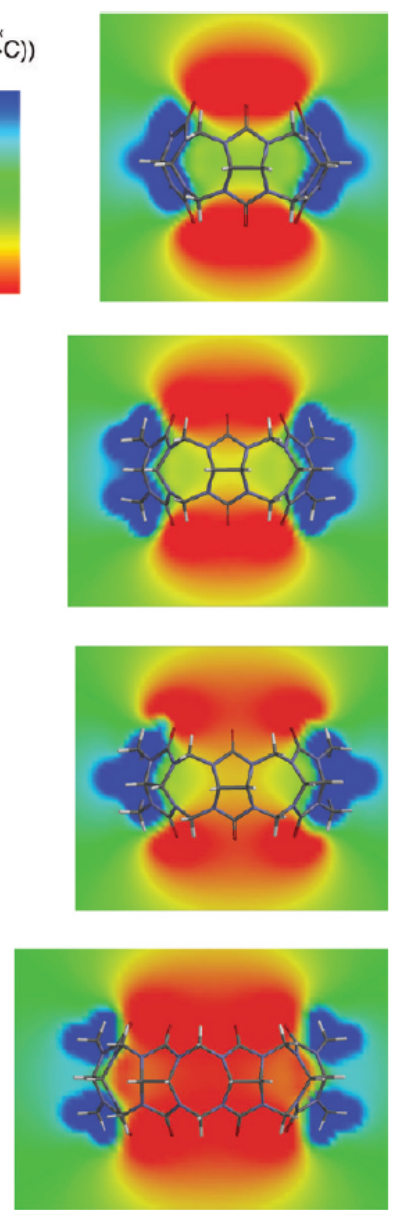

Figure 3: Calculated electrostatic potential (EP) for (a) CB[5], (b) CB[6], (c) CB[7], and (d) CB[8]. Reprinted with permission from Biedermann, F. \& Scherman, O. A. J. Phys. Chem. B 116, 2842-2849 (2012). Copyright 2012 American Chemical Society. 
tend to be dicationic species where the cations are separated by an organic/hydrophobic region. Examples of such strong binders for $\mathrm{CB}[6], \mathrm{CB}[7]$ and $\mathrm{CB}[8]$ include cadaverine, methylviologen and amino-adamantane, respectively, with the size of the hydrophobic region being tailored for the specific volume of each $\mathrm{CB}$ cavity.

Table 1: Structural parameters for uncomplexed CB[5]-CB[8]. ${ }^{a}$ Adapted with permission from Kim, J. et al. J. Am. Chem. Soc. 122, 540-541 (2000). Copyright 2000 American Chemical Society. ${ }^{b}$ Reproduced from Ref. ${ }^{39}$ with permission from The Royal Society of Chemistry.

\begin{tabular}{|l|l|l|l|l|l|}
\hline & $\mathrm{CB}[5]^{a}$ & $\mathrm{CB}[6]^{a}$ & $\mathrm{CB}[7]^{a}$ & $\mathrm{CB}[8]^{a}$ & $\mathrm{CB}[10]^{b}$ \\
\hline portal diameter $(\AA)$ & 2.4 & 3.9 & 5.4 & 6.9 & $9.5-10.6$ \\
\hline cavity diameter $(\AA)$ & 4.4 & 5.8 & 7.3 & 8.8 & $11.3-12.4$ \\
\hline cavity volume $\left(\AA^{3}\right)$ & 82 & 164 & 279 & 479 & 870 \\
\hline outer diameter $(\AA)$ & 13.1 & 14.4 & 16.0 & 17.5 & 20.0 \\
\hline height $(\AA)$ & 9.1 & 9.1 & 9.1 & 9.1 & 9.1 \\
\hline
\end{tabular}

Another facet to the binding of guest species within $\mathrm{CB}[n]$ cavities is how well a guest physically fits within the cavity. A good predictor of $\mathrm{CB}[n]$ complex binding comes from the assessment of whether the inner cavity of the host and the shape of the guest display good complementarity. The cavity volumes of $\mathrm{CB}[5]-\mathrm{CB}[8]$ are shown in Table 1 and range from $82 \AA^{3}$ for $\mathrm{CB}[5]$, to $479 \AA^{3}$ for $\mathrm{CB}[8] .{ }^{24}$ As a result of its small size, $\mathrm{CB}[5]$ is mostly suitable for the encapsulation of gases (discussed in Section 7), whereas CB[6] can bind alkyl chains, $\mathrm{CB}[7]$ can accommodate small aromatic compounds and $\mathrm{CB}[8]$ can simultaneously complex with two molecules, suggesting that guest size is an important factor when considering $\mathrm{CB}[\mathrm{n}]$ :guest complexes. This steric aspect of molecular host-guest recognition has been quantified by Mecozzi et al. ${ }^{40}$ and termed the packing coefficient. Packing coefficients are defined as the ratio of guest volume to the volume of the host cavity, and this principle has been successfully applied to predict complexation between $\mathrm{CB}[5]-\mathrm{CB}[8]$ with appropriate guest compounds. ${ }^{41}$

The distinct advantage of employing $\mathrm{CB}[n] \mathrm{s}$ in host-guest molecular recognition is that complexes involving $\mathrm{CB}[n]$ can be formed in water with high binding affinity. Typically, aqueous environments cause a drop in host-guest affinity, compared to organic sol- 
vents, as water can compete strongly for hydrogen bonds and efficiently solvate charged species. $\mathrm{CB}[\mathrm{n}] \mathrm{s}$ overcome this issue via the presence of high energy water, that is present in the CB[n] cavity, which supplies a driving force for guest complexation. ${ }^{42-44}$ The release of high energy water and its role in macrocycle complex formation has been comprehensively reviewed (Figure 4$).{ }^{45}$

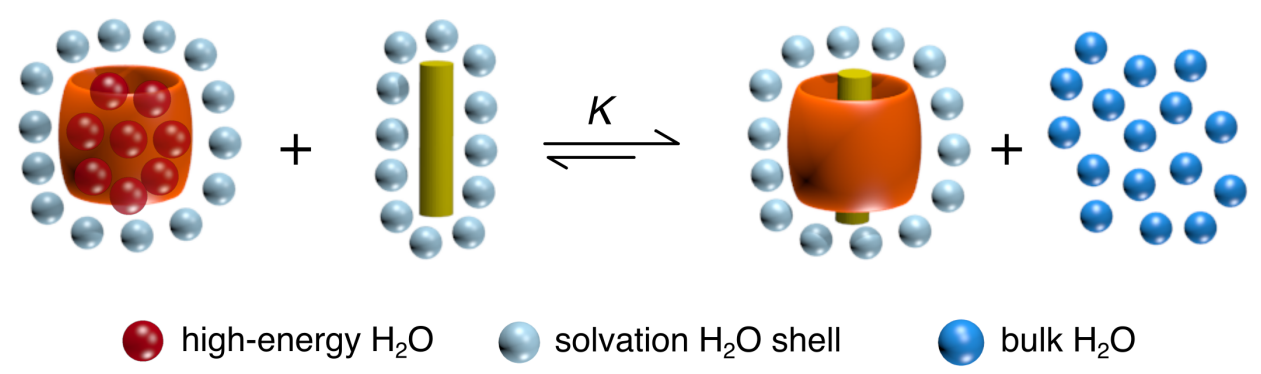

Figure 4: Schematic illustration of the formation of $\mathrm{CB}[n]$ host-guest complex driven by the release of high-energy water. Adapted with permission from Biedermann et al. J. Am. Chem. Soc. 2012134 1531815323. ${ }^{42}$ Copyright 2012 American Chemical Society.

All cavities larger than a critical size contain water molecules, for entropic reasons, even if the breaking of hydrogen bonds is necessary. Such water molecules experience a reduced number of hydrogen bonds compared to bulk water, and are thus of high energy. For the case of $\mathrm{CB}[n] \mathrm{s}$, the binding of a guest molecule releases the energetically frustrated water molecules, which lowers the energy of the system, providing an enthalphic and entropic gain in favour of complex formation. This hydrophobic effect has been studied using molecular dynamics simulations, ${ }^{43}$ where it was determined that host-guest affinity is higher for cases where all water molecules are removed from the cavity (related to the packing coefficient) and where the encapsulated water is of high energy. CB[7] was shown to result in the largest energy gain from water expulsion of the whole $\mathrm{CB}[n]$ family, as it contains more water molecules than $\mathrm{CB}[5]$ and $\mathrm{CB}[6]$, and has higher energy water molecules encapsulated than $\mathrm{CB}[8]$, as the cavity size of $\mathrm{CB}[8]$ allows for encapsulated water to be structurally organised in a manner more closely resembling bulk water.

Ion-dipole binding, host-guest packing coefficients and hydrophobic effects all need to 
be taken into account when considering guest molecules for $\mathrm{CB}[n]$ complexing with high binding affinity. As a follow up to this section, binding tables can be found in Section 14 , that provide an extensive list of the guest species for $\mathrm{CB}[5]-\mathrm{CB}[8]$, along with their binding constants $(\log K)$. This material should provide the reader with an understanding of the principles behind $\mathrm{CB}[n]$ complex formation, in addition to a comparative list of $\mathrm{CB}[n]$ guests.

\section{Specific binding properties of $\mathrm{CB}[5]$}

$\mathrm{CB}[5]$ is the smallest macrocycle in the cucurbit $[n]$ uril family. With its synthesis and isolation first reported by Kim et al. in the year 2000, it has a portal diameter of $2.4 \AA$, and a cavity volume of $82 \AA^{3}$ (which is under half the volume of $\mathrm{CB}[6]$ ). ${ }^{4}$ As a result of its small cavity size, $\mathrm{CB}[5]$ has found limited use in the encapsulation of guest molecules compared to the larger homologues, however, the carbonyl groups of the glycoluril sub-units allow for the binding of cationic species to the portals of $\mathrm{CB}[5] .^{29,34,46}$ It has been shown that protons can bind to the portals of $\mathrm{CB}[n] \mathrm{s}$, and indeed this is the mechanism by which the more insoluble CBs can be dissolved through the use of aqueous acidic media. ${ }^{34} \mathrm{CB}[5]$ and decamethyl-CB[5] have also been shown to form portal complexes with alkali, alkaline earth and ammonium cations, ${ }^{46}$ in addition to hexamethylenetetramine, which can act as a lid on the carbonyl portals of $\mathrm{CB}[5] .{ }^{47} \mathrm{CB}[5]$ and decamethyl-CB[5] form very weak complexes with $\alpha, \beta$ and $\gamma$ forms of cyclodextrin, with binding constants ranging between $\log \mathrm{K}=0.74$ and $1.34 .{ }^{48} \mathrm{CB}[5]$ also exhibits binding with multi-charged cations including $\mathrm{Co}^{2+}, \mathrm{Ni}^{2+}, \mathrm{Cu}^{2+}, \mathrm{Pb}^{2+}, \mathrm{Zn}^{2+}, \mathrm{Cd}^{2+}, \mathrm{Cr}^{2+}$ and $\mathrm{Fe}^{2+} .29$

$\mathrm{CB}[5]$ shares similar portal binding properties with larger members of the cucurbituril family, however, its small internal cavity limits its ability to support host-guest complex formation. Even though $\mathrm{CB}[5]$ is not big enough to incorporate large molecules into its cavity, its small size gives rise to its niche: the encapsulation of gaseous molecules. 
Decamethyl-CB[5] has been demonstrated to encapsulate small gaseous species in both aqueous environments and in solid form. Gases such as $\mathrm{N}_{2}, \mathrm{O}_{2}, \mathrm{Ar}, \mathrm{N}_{2} \mathrm{O}, \mathrm{CO}$ and $\mathrm{CO}_{2}$ have been shown to be encapsulated in both powdered and aqueous decamethyl-CB[5], whilst $\mathrm{He}, \mathrm{Ne}, \mathrm{H}_{2}, \mathrm{Kr}, \mathrm{Xe}$ and $\mathrm{CH}_{4}$ could only be encapsulated in an aqueous environment, with $\mathrm{Kr}, \mathrm{Xe}$ and $\mathrm{CH}_{4}$ requiring heating of the sample to assist encapsulation. ${ }^{49} \mathrm{~A}$ full discussion of the gas sorption properties of cucurbit[n]urils is offered in section 7 .

\section{Specific binding properties of $\mathrm{CB}[6]$}

$\mathrm{CB}[6]$ is the most abundant homologue and with a portal diameter of $3.9 \AA$, it is commonly known to form stable complexes with aliphatic amines. The insolubility of $\mathrm{CB}[6]$ in organic solvents and sparing solubility in water has, however, had a limiting impact on its applications in its unmodified form. This is evident in the different solvent conditions in which the binding of $\mathrm{CB}[6]$ complexes are often reported. For example, Mock and coworkers first studied the complexation of a variety of alkyl- and aryl-substituted alkylammonium and alkyldiammonium ions in aqueous formic acid. ${ }^{50}$ The results highlighted the size and shape complementarity exhibited by $\mathrm{CB}[6]$ contributing to its selective binding properties. It is well-established that the stability of these complexes are a result of strong interactions between the positively charged protonated amines and the six electronegative carbonyl moieties at the portals. The hydrophobic nature of the methylene groups favor their positioning inside the cavity of the $\mathrm{CB}[6]$ where the solvent water molecules are excluded, adding to further stabilization of the complexes. Therefore, the most stable complex is formed for an optimal length of the aliphatic chain such that the amine groups are positioned in close proximity to the portal groups. As a result, CB[6] shows strongest binding to pentano and hexano bridged $\alpha, \omega$-diammonium functionalities. The binding properties of $\mathrm{CB}[6]$ with imidazolium-based guests have also been investigated widely ${ }^{37,38,51}$ and has been used in the synthesis of monofunctionalised $\mathrm{CB}[6]^{8}$ 
as well as in the isolation of $\mathrm{CB}[n]$ homologues. ${ }^{52}$

The importance of the surroundings of the complex, however, has a major impact on the strength of the resulting complex as demonstrated by Lee et al. ${ }^{53}$ In this correlation study of the binding behavior of diammonium guests between solution phase $(50 \% \mathrm{HCOOH}, \mathrm{ITC})$ and gas phase (ESI-MS) it is shown that while a correlation exists between the two phases, the stabilities of the complexes are different. They suggest that this contrast is a result of an additional role played by the H-bonding with water molecules in the solution phase, while only ion-dipole interactions predominantly exist in the gas phase. In the gas phase, the guest has to undergo a structural distortion in order to maximize ion-dipole interactions with the portal. The distortion is not needed in water because it is compensated in solution through hydrogen bonding with water.

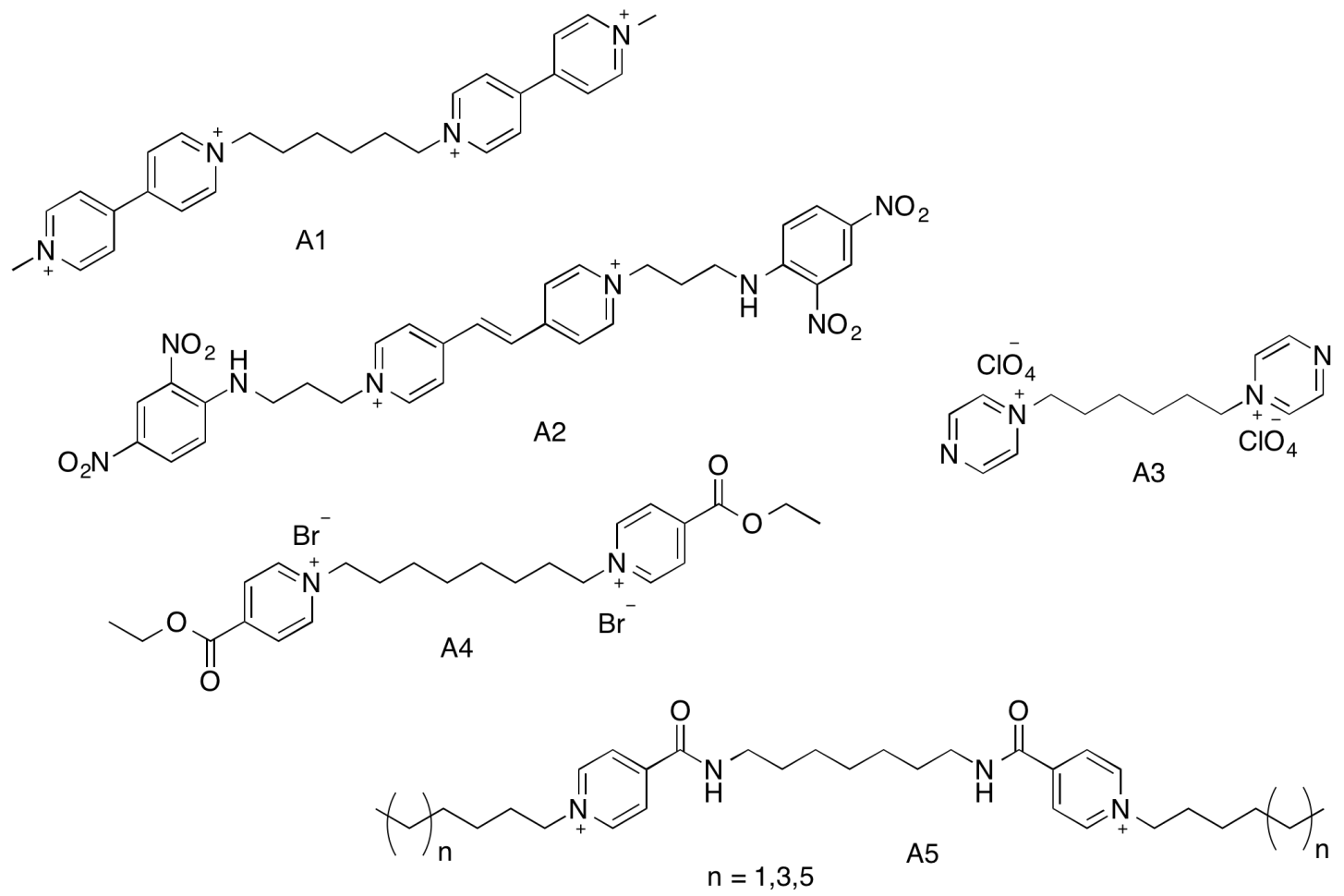

Figure 5: Pyridinium based structures for the formation of rotaxane and pseudorotaxanes with CB[6].

The high binding affinities of $\mathrm{CB}[6]$ towards aliphatic amines compared to aromatic groups has led to various rotaxane and pseudorotaxane constructs, where the alkylamines act as an axle and the $\mathrm{CB}[6]$, the wheel such as with 1,8-diaminooctane. ${ }^{54}$ A 1:1:1 system 
with $\mathrm{CB}[6]$ and cyclodextrin using a dihexylammonium guest has also been reported. ${ }^{55}$ Threads containing pyridinium and imidazolium moieties have also been widely reported in contructing pseusorotaxanes and rotaxanes with $\mathrm{CB}[6]$. For example, a [2]pseudorotaxane system was reported using structure A1, where the host is located over the hexamethyl chain of the guest. In a dynamic rotaxane derived from a $\mathrm{CB}[6]$ complex with a bispyridinium guest (A2), $\mathrm{CB}[6]$ is able to shuttle between the two pyridinium groups. ${ }^{56}$ Self-assembly of pseudorotaxanes constructed with $\mathrm{A} 3 \cdot \mathrm{CB}[6]^{57}$ and $\mathrm{A} 4 \cdot \mathrm{CB}[6]^{58}$ have been reported to form infinite hydrogen-bonded one-dimensional and two-dimensional networks. Other high order rotaxane-based structural frameworks have also been reported, ${ }^{59}$ including polypseudorotaxanes. ${ }^{60,61} \mathrm{He}$ et al. have reported the formation of pseudorotaxanes with a series of bis-pyridinium compounds (see A5 in Figure 5), where the binding site of $\mathrm{CB}[6]$ could be tailored by increasing length of the chains at the two ends of the guest. ${ }^{62}$

Several [3] rotaxanes have been reported based on imidazolium containing structures $\left(\mathbf{A} 6^{64}\right.$ and $\left.A 7^{65}\right)$. Recently, photo-responsive dithienylethene-based rotaxanes (A8) were constructed with $\mathrm{CB}[6] .{ }^{66} \mathrm{CB}[6]$ has also been used to form water soluble [5]rotaxane and [5]pseudorotaxane using a meso-tetraphenyl porphyrin (see A9 in Figure 6) with $\mathrm{pH}$ driven switchable properties. ${ }^{63}$

These selective recognition properties of $\mathrm{CB}[6]$ were first ultilized by Kim and coworkers in order to construct a fluorescent molecular switch based on a pseudo-rotaxane structure. ${ }^{67}$ Various fluorescent sensors have been reported using the indicator-displacement strategy with dye molecules derivatized with diamino-alkyl derivatives (see A10-A13 in Figure 7), whereby the alkyl moiety anchors strongly inside the CB[6] cavity. ${ }^{68-70}$ Recently, the fluorescence emission of pyrene was reported to get enhanced in the presence of $\mathrm{CB}[6]$ as a result of external supramolecular interactions between pyrene and $\mathrm{CB}[6]{ }^{71}$ $\mathrm{CB}[6]$ has also been used for the detection of 3,4-methylenedioxymethamphetamine, the active constituent in ecstasy samples, using cyclic voltammetry. ${ }^{72}$ 

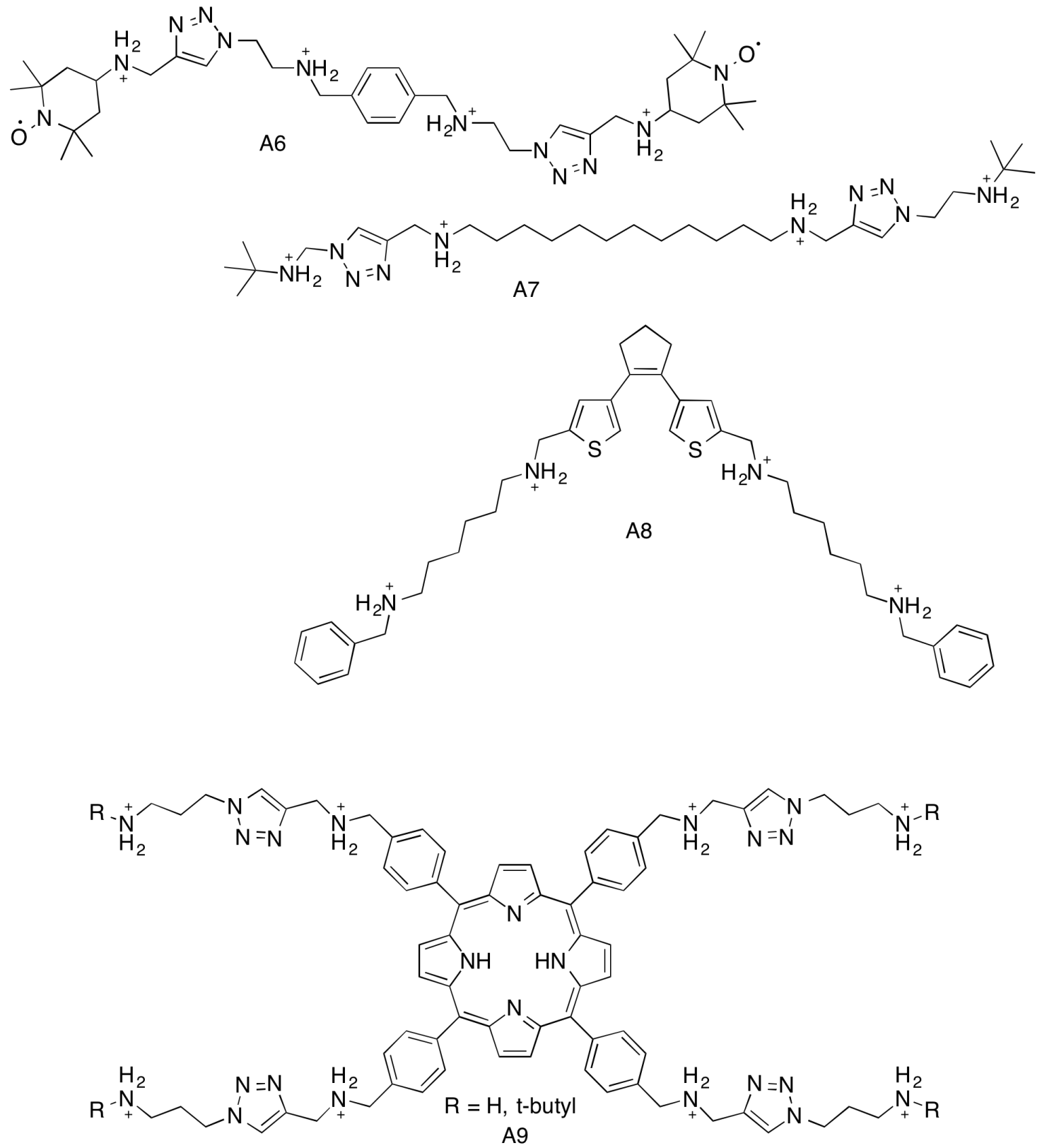

Figure 6: Representative structures for the formation of rotaxane and pseudorotaxanes with $\mathrm{CB}[6] .{ }^{63}$ 


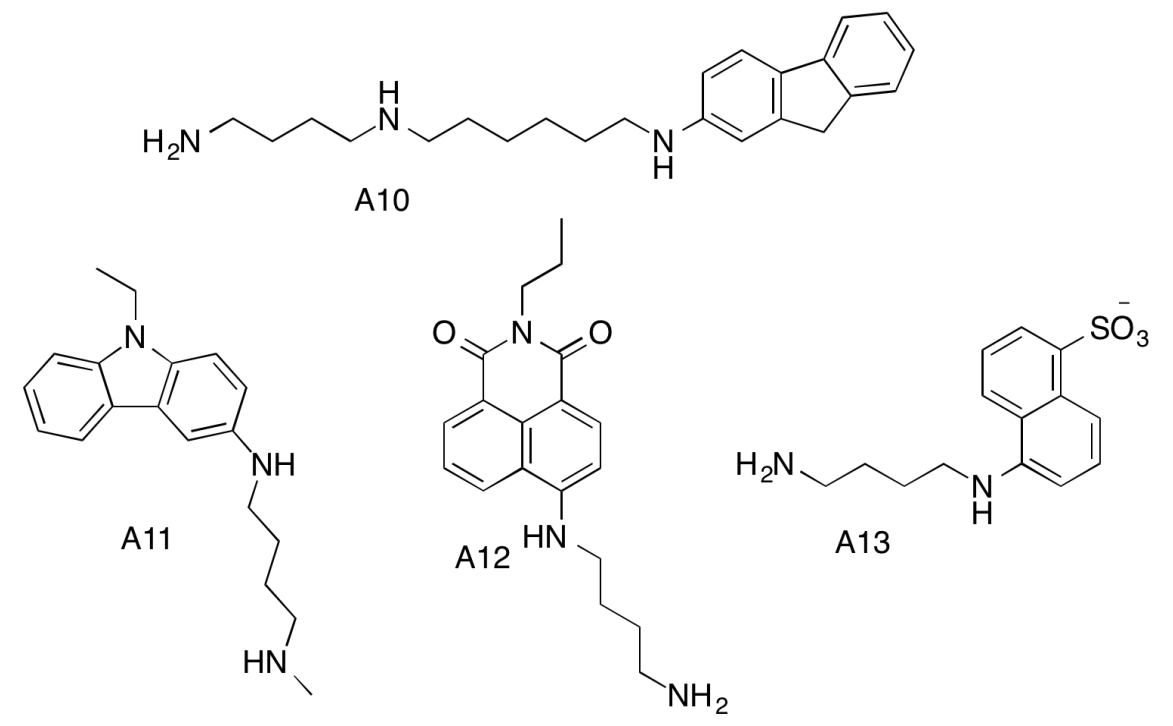

Figure 7: Alkyldiammonium-dye conjugates used for fluorescence sensor designs with CB[6] ${ }^{67-70}$

Catecholamines such as adrenaline ${ }^{73}$ and isoprenaline ${ }^{74}$ show distinct binding behavior between $\mathrm{CB}[6]$. In particular, a striking transformation of the crystals of the complex from needle-like to prismatic structures can be observed over a period of a few days (Figure $8 \mathrm{a}-\mathrm{c}) .{ }^{73}$ This is attributed to the formation of a $1: 1 \mathrm{CB}[6] \cdot$ adrenaline complex as a kinetic product, which slowly evolves to form a 2:1 thermodynamically favored complex (Figure $8 \mathrm{~d}, \mathrm{e}$ ). Furthermore, in the same study, binding between dimerized adrenaline and $\mathrm{CB}[6]$ in acidic media was also reported (Figure 8f).

Characterization of the binding parameters for $\mathrm{CB}[6]$ and its guests is usually challenging in water owing to the poor aqueous solubility of $\mathrm{CB}[6]$. A remarkable number of crystal structures, however, have been reported for CB[6] guest complexes, which provide important structural insights into the complexed adduct formation. While the binding of $\mathrm{CB}[6]$ with aromatic compounds is surprising because of its smaller cavity diameter, it is noteworthy that arylamines with suitable substituents are known to become encapsulated within the $\mathrm{CB}[6]$ cavity. ${ }^{50}$ This remarkable observation is particularly clear in the crystallographic structures reported for $\mathrm{CB}[6] \cdot p$-xylylenediammonium ion adducts, whereby an ellipsoidal distortion of the host molecule upon guest encapsulation is clearly observed, ${ }^{75,76}$ however differences may exist between solid and aqueous forms of this 
complex. Although $\mathrm{CB}[6]$ is typically unable to accommodate six-membered aromatic rings, five membered aromatic rings such as thiophenes ${ }^{50}$ and furans ${ }^{77}$ are able to bind to $\mathrm{CB}[6]$.
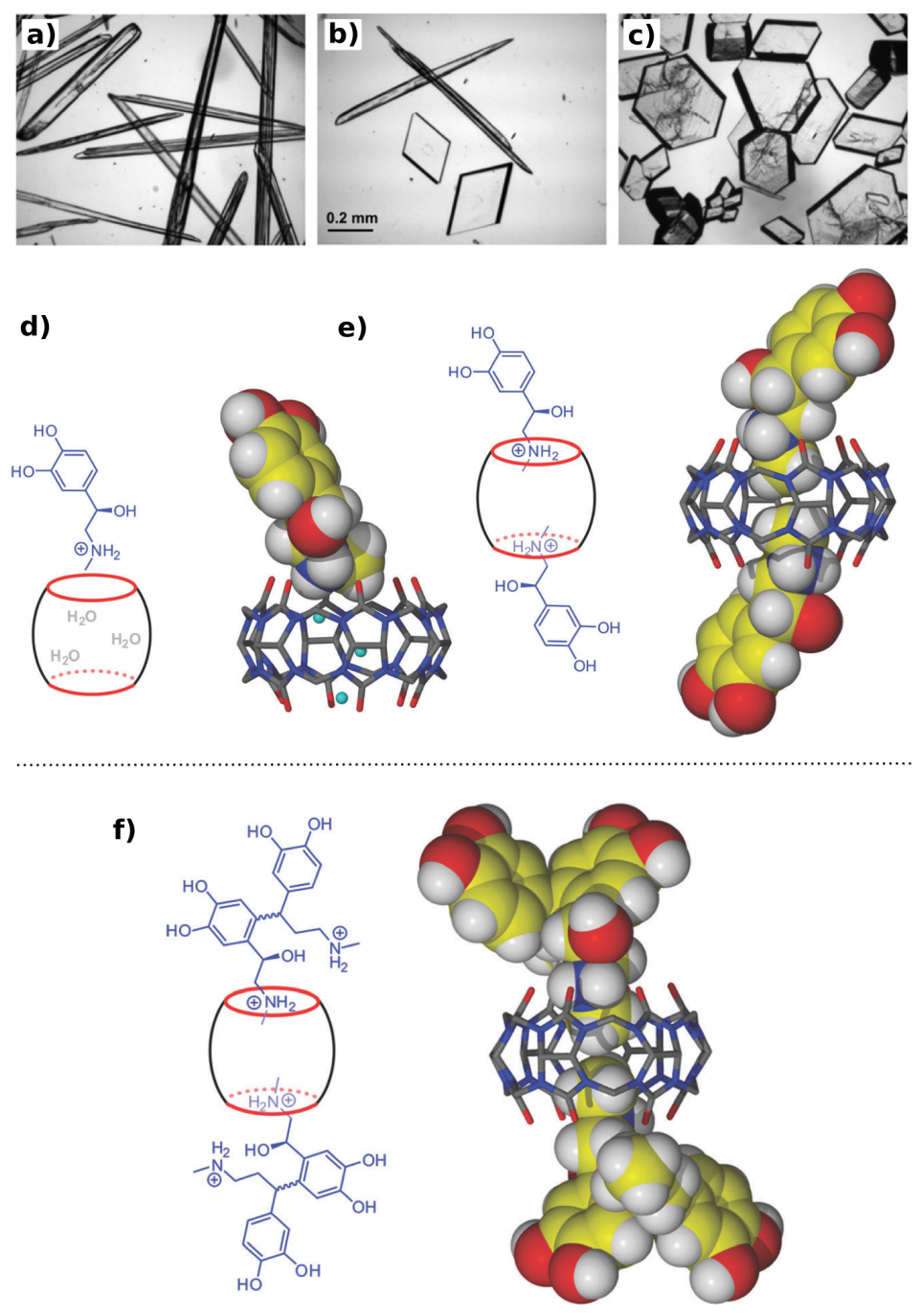

Figure 8: Pictures of crystals of $\mathrm{CB}[6]$-adrenaline complexes after (a) 1 hour showing needle-like crystals; (b) 2 days both needles and prisms; (c) 1 week only prisms. Schematic representation and crystal structures of different complexation modes of CB[6]·adrenaline showing (d) 1:1 kinetic complex, (e)1:2 thermodynamic complex and (f) dimerized adrenaline under acidic medium. Figure adapted with permissions from Danylyuk et al, Chem. Commun. 2013, 49, 1859-1861; All images reproduced with permission from The Royal Society of Chemistry.

$\mathrm{CB}[6]$ is widely applied in separation science. For example, in a recent study, monolithic poly(glycidyl methacrylate-co-ethylene dimethacrylate) capillary columns were incorporated with $\mathrm{CB}[6]$ monorotaxanes for application in polymer monolith microextrac- 
tion to separate and preconcentrate nitroaromatics. These monoliths were used for trace analysis of nitroaromatic compounds in blood and water with 83 to 107 percent recovery. ${ }^{78}$ In another study, a new type of stationary phase was constructed using $\mathrm{CB}[6]$ in combination with guanidinium-based ionic liquid. ${ }^{79}$

The ability of $\mathrm{CB}[n]$ to modulate chemical reactions has been known since the first report of the acceleration of 1,3-dipolar cycloaddition reaction under the catalytic influence of $\mathrm{CB}[6]$ reported by Mock et al. ${ }^{3}$ Moreover, using the inclusion of bromine and iodine were previously reported by Nau and co-workers, ${ }^{80}$ Reddy et al have shown the application of these halogen inclusion complexes such as $\mathrm{I}_{2}-\mathrm{CB}[6]$ for catalytic reactions and $\mathrm{Br}_{2}-$ $\mathrm{CB}[6]$ to achieve electrophilic bromination of benzene and formation of bromohydrin. ${ }^{81}$

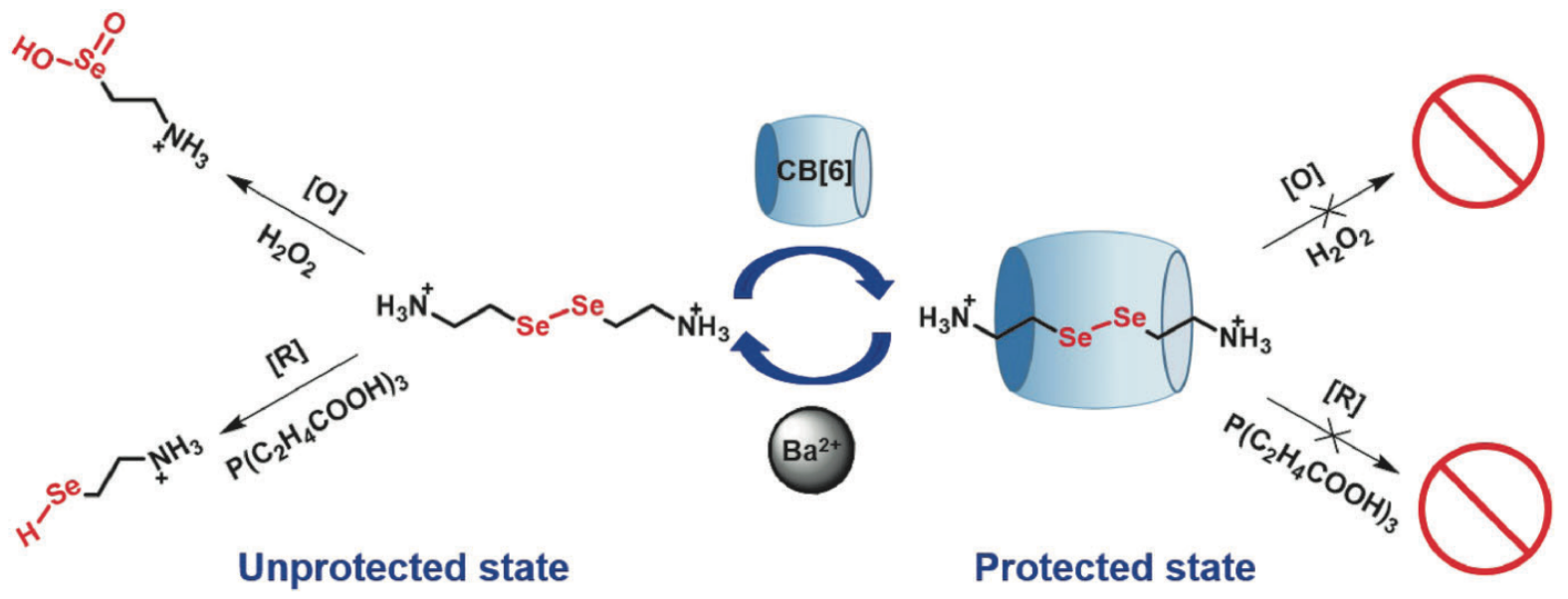

Figure 9: Protection and deprotection of selenocystamine achieved by controlled supramolecular complexation with CB[6]. Reproduced from Ren et al, ChemPhysChem 2015, 16, 523527, with permission from John Wiley and sons, Inc.

Recently, the role of $\mathrm{CB}[6]$ in the protection of selenocystamine was demonstrated against redox stimuli (Figure 9). The Se-Se bonds were shown to be stable in the presence of $\mathrm{CB}[6]$ when exposed to $\mathrm{H}_{2} \mathrm{O}_{2}$, a strong oxidizing agent and tris(2-carboxyethyl)phosphine, a strong reductant. $\mathrm{CB}[6]$ is able to modulate the reaction of the selenocystamine by encapsulating the Se-Se bonds inside its cavity. Selenocystamine forms a strong host-guest complex with $\mathrm{CB}[6]$ under acidic conditions ( $\mathrm{pH}$ 5.8) with a $\log \mathrm{K}$ value of 6.7 at $25^{\circ} \mathrm{C}$ as 
measured by ITC. The protection behavior was shown to be applicable over a wide range of $\mathrm{pH}$ values (up to 11.8). Furthermore, the reactivity of selenocystamine guest could be fully recovered through dissociation with $\mathrm{CB}[6]$ by adding $\mathrm{BaCl}_{2} \cdot{ }^{82}$ The ability of $\mathrm{CB}[6]$ to catalyze and template reactions will be discussed in greater detail in Section 11.

Several $\mathrm{CB}[6]$ ion adducts have been studied recently. ${ }^{83-90} \mathrm{Cd}(\mathrm{II}) \cdot \mathrm{CB}[6]$ complexes have been studied comprehensively in an electrochemical study through competitive binding with acetic acid, isobutylamine and 1,5 diaminopentane. ${ }^{86}$ The use of $\mathrm{CB}[6]$ ion complexes are also emerging in materials chemistry, for example, as $\mathrm{Sr}(\mathrm{II}) \cdot \mathrm{CB}[6]$ based polymers ${ }^{87}$ and hexachloroplatinate (IV) anion. $\mathrm{CB}[6]$ based porous materials have been reported. ${ }^{88}$ Theury et al. reported the formation of $\mathrm{La}^{+}$ammoniocarboxylate complexes with $\mathrm{CB}[6] .{ }^{83}$ Later, the luminescent properties of $\mathrm{CB}[6]$ coordination compounds with $\mathrm{La}^{+}(\mathrm{Eu}, \mathrm{Sm}, \mathrm{Tb}$ and $\mathrm{Tm})$ were also investigated in acidic aqueous media. ${ }^{89} \mathrm{In}$ another study, a new mononuclear dysprosium(III)-cucurbit[6]uril complex was synthesized and characterized structurally and magnetically. ${ }^{90}$ Chen et al. have also reported the $\mathrm{CB}[6]-$ based capture of $\mathrm{Cs}^{+}$cations. ${ }^{91}$

The binding of ions such as alkali metals at the portals of the $\mathrm{CB}[6]$ have been wellknown. In a previous study investigating the properties of $\mathrm{CB}[6]$ as a sorptive material for (textile) dyes, the presence of cations such as $\mathrm{Ca}^{2+}$ or $\mathrm{Sr}^{2+}$ enhanced the binding of the dyes to $\mathrm{CB}[6] .{ }^{92}$ On the contrary, in a study with methyl viologen and $\mathrm{CB}[6]$, where methyl viologen is only able to interact with $\mathrm{CB}[6]$ externally, the presence of salt even hindered these weak interactions between them. ${ }^{93}$ Molecular dynamic simulations also show evidence of the preferential formation of $\mathrm{CB}[6] \cdot \mathrm{Na}^{+}$complexes in the presence of hexamethylene diammonium cations in saline solutions. ${ }^{94}$ Therefore, while the introduction of ions is often necessary in order to enhance the solubility of $\mathrm{CB}$ [6] in water, their role as an interferent between $\mathrm{CB}[6]$ and binding with other organic guests cannot be ignored. 


\section{Specific binding properties of $\mathrm{CB}[7]$}

$\mathrm{CB}[7]$, the third member of the macrocyclic family, is large enough to form inclusion complexes with guest molecules that are typically bulkier than simple aliphatic chains and gas molecules that are usually encapsulated by the smaller homologues, $\mathrm{CB}[5]$ and $\mathrm{CB}[6] .{ }^{15}$ On account of it's larger cavity size relative to $\mathrm{CB}[6]$, a much wider range of guests can be recognized by $\mathrm{CB}[7]$ and encapsulated within, often exhibiting highly favorable binding parameters, see Table 5. Specifically, CB[7] has a portal diameter of $5.4 \AA$ and cavity diameter of $7.3 \AA$, see Table $1 .{ }^{95}$ This affords CB[7] an inner cavity volume of $279 \AA^{3}$, large enough for the inclusion of simple three dimensional molecules such as ferrocene and adamantylamine derivatives (see Figure 10 for examples), accompanied by high binding constants ( $\log K>12)$. This occurs through excellent cavity filling effects, expelling all 'high energy' water molecules from the macrocycle core. ${ }^{41}$ These particularly impressive binding constants are undoubtedly one reason for the large number of reports in recent literature, with over 90 individual publications in 2014 alone.

Not only does CB[7] have a cavity size amenable to the encapsulation of sizeable guest molecules, it also has a much greater aqueous solubility relative to other members of the $\mathrm{CB}[n]$ family, exhibiting a water solubility $30 \mathrm{mM}$ compared to $0.01 \mathrm{mM}$ for $\mathrm{CB}[8]$ (also large enough to encapsulate 3D guest molecules). ${ }^{15}$ This greater solubility, coupled with the larger cavity volume has resulted in a variety of aqueous specific applications of $\mathrm{CB}$ [7], particularly in drug encapsulation as described in a recent review. ${ }^{18}$ Drug encapsulation is not the only biomedical area where CB[7] has been utilized, numerous reports from Urbach and others have highlighted binding of $\mathrm{CB}[7]$ to peptides, proteins, biomolecules (i.e. neurotransmitters) and dyes, demonstrating applicability to highly accurate bio-sensing applications at sub-nanomolar concentrations. ${ }^{96-98} \mathrm{CB}[7]$ 's solubility at usual drug therapeutic levels has also leant the macrocycle toward a wide range of toxicity assessing studies, from in vitro studies to mouse and zebrafish models. ${ }^{99-102} \mathrm{Al}-$ though $\mathrm{CB}[7]$ is not the perfect representation of biocompatibility for all other members of 

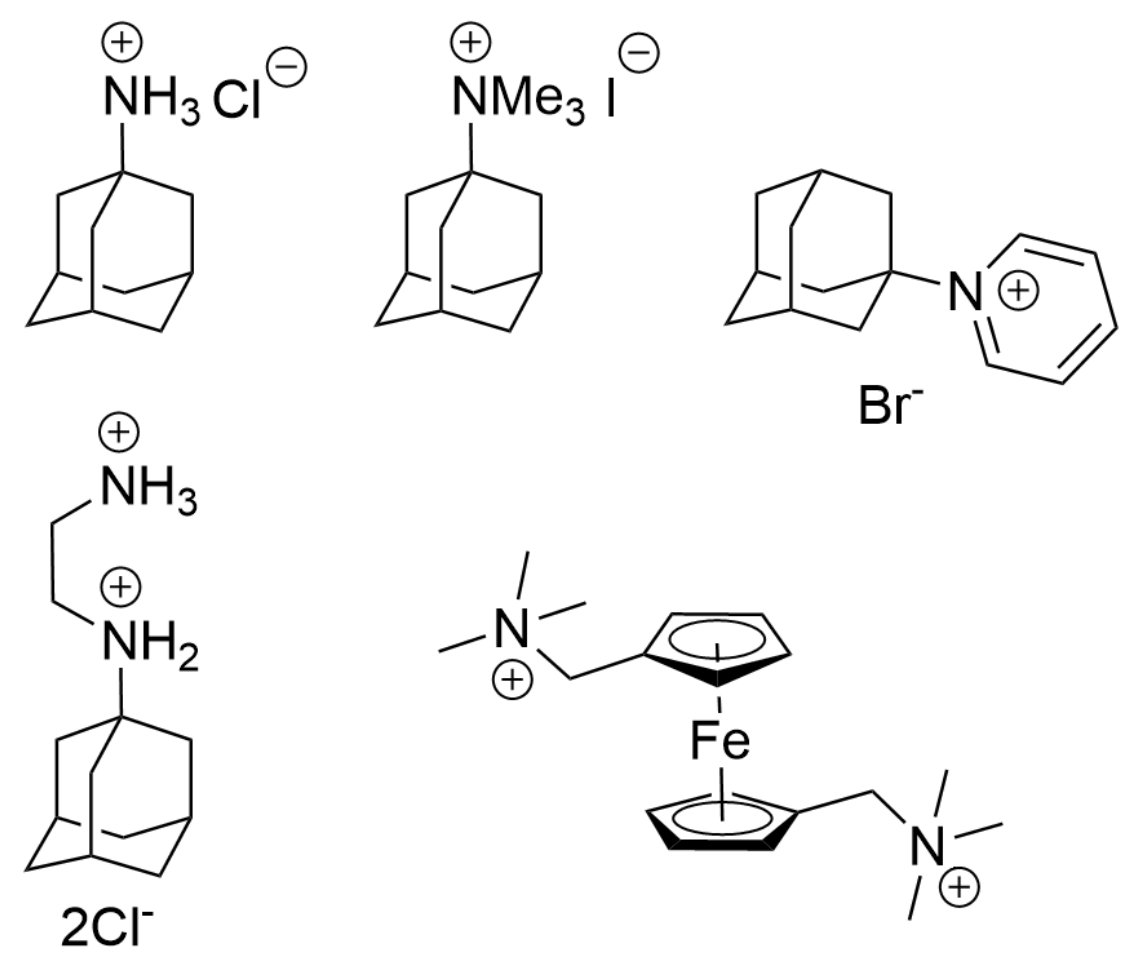

Figure 10: Examples of first guests with high binding constants for CB[7].

the $\mathrm{CB}[n]$ family, the good degree of tolerability demonstrated in these studies is promising.

Because of these highly favorable binding equilibria, $\mathrm{CB}[7]$ has found other unique uses, ranging from the assembly of supramolecular energy transfer motifs, stimuli responsive underwater adhesives, and catalysis. ${ }^{17,103,104} \mathrm{CB}[7]$ literature has dramatically expanded over the past decade and potential applications of the macrocycle are continuously emerging. We expect to see vast progress and efforts in the area of controlled functionalization and attachment of $\mathrm{CB}[7]$ receptors to a variety of materials, whereby the $\mathrm{CB}[7]$ can be isolated within a material still allowing for high affinity binding. ${ }^{105}$ 


\section{Specific binding properties of $\mathrm{CB}[8]$}

The cavity volume of $\mathrm{CB}[8]$ is $479 \AA^{3}$, which is similar to $\gamma$-CD and 1.7 times larger than that of $\mathrm{CB}[7]$, and its binding properties parallel in many ways those of $\mathrm{CB}[5]-$ $\mathrm{CB}[7] .{ }^{15} \mathrm{CB}[8]$ displays relatively strong binding affinities towards bulky amphiphilic positively charged guests including adamantane and ferrocene derivatives C1-C5 (binding constants were determined by competition with melamine-derived guest $\mathbf{C} \mathbf{6}$ for a limiting quantity of $\mathrm{CB}[8]$, see Figure 11 and Table 6). ${ }^{106}$ It can also encapsulate larger macrocyclic guests including cyclen(1,4,7,10-tetra/-aza/-cyclo/-do/-decane) and cyclam (1,4,8,11-tetra/-aza/-cyclo/-tetra/-decane) (C7 and C8 in Figure 11), thus allowing formation of a "macrocycle within macrocycle" complex. ${ }^{13}$ Kim and coworkers were able to metalate the bound tetraaza macrocycles with $\mathrm{Cu}$ (II) and $\mathrm{Zn}$ (II) thus yielding Russian doll type metal ion complexes. ${ }^{107,108}$ This strategy has been further exploited to prepare a large number of inclusion compounds of transition metal complexes including nickel (II). ${ }^{109-111}$ The inclusion of the dimethylated cyclen C9 (Figure 11) has also been reported. ${ }^{112} \mathrm{CB}[8]$ has even shown the ability to bind larger guests such as fullerene, forming complexes with two fullerene guests bound externally to the $\mathrm{CB}[8]$ portals, one being bound to each portal. ${ }^{113}$ In the presence of $\mathrm{CB}[8]$, alkylammonium salts equipped with long aliphatic chains, C10-C15, (Figure 11) bend and are incorporated inside the cavity of the host by adopting a U-shaped conformation. ${ }^{114,115}$ When the same guests are in the presence of the smaller $\mathrm{CB}[7]$, only the signals corresponding to the methylene protons near the charged group shift in the ${ }^{1} \mathrm{H}$ NMR of the complex while the signals of the protons at the terminal end of the guest chain, far away from the charged group, remains unchanged. This suggests that the $\mathrm{CB}[7]$ sits nearby the ammonium group with the other end of the guest sticking out of the macrocycle cavity. On the contrary, all the methylene proton signals are shifted when in the presence of $\mathrm{CB}[8]$, suggesting that the larger width of the $\mathrm{CB}[8]$ cavity allows the aliphatic chain to form a U-shape so that the entire guest can be encapsulated. The U-shaped conformation of the guest inside the cavity of $\mathrm{CB}[8]$ was 
supported by $x$-ray crystal structures and ROESY NMR. The authors suggested that van der Waals contact between the hydrophobic chain and the cavity can be the main cause of the significantly large enthalpic gain over compensating the entropic penalty associated to the conformational restriction of the U-shaped aliphatic chain. It has been nevertheless suggested that dispersion interactions inside the $\mathrm{CB}[n]$ cavity do not present a significant enthalpic driving force for guest inclusion. ${ }^{35}$ Other doubly charged alkylammonium salts including 1,4-butylidene and 1,10-decylidenedipyridinium (C16 and C17 in Figure 11) and 1,12-dodecane diammonium (C18 in Figure 11), in the presence of one equivalent of $\mathrm{CB}$ [8], also exhibit the previously described U-shaped folding of C10-C15. ${ }^{116,117}$ In the case of $\mathbf{C 1 8}$, both charged groups bind to the same $\mathrm{CB}[8]$ portal with one water molecule bridging the two ammonium groups.

The encapsulation of nitroxide derivatives inside $\mathrm{CB}[8]$ has recently been described on several occasions by Kaifer, Lucarini, Tordo, Ouari, Turro, Ramamurthy and others. ${ }^{118-127}$ Lucarini and coworkers reported in 2009 the encapsulation of an amino-TEMPO nitroxide derivative, $\mathrm{C} 19$, inside $\mathrm{CB}[8]$ and observed changes in the nitrogen hyperfine splitting by Electron Paramagneting Resonance (EPR), which were attributed to the formation of a host-guest complex. ${ }^{118}$ Furthermore, cooling of an aqueous solution of CB[8].C19 from room temperature to $5{ }^{\circ} \mathrm{C}$ led to the formation of a macroscopic fibrous network, which exhibited the characteristic EPR spectrum of a polyradical (solid fibers). The authors suggested the formation of the supramolecular ternary radical $\mathrm{CB}[8]_{3} \cdot \mathrm{C} 19_{3}$ to explain the EPR spectrum of the fibers dissolved in a $\mathrm{NaCl}(1 \mathrm{mM})$ aqueous solution at room temperature. The existence of a supramolecular ternary radical was demonstrated by Tordo, Ouari and coworkers, who used nitroxide $\mathbf{C 2 0}$ as a probe to investigate the binding properties of $\mathrm{CB}[8] .{ }^{119}$ These authors established the concentration-dependent aggregation behavior of $\mathrm{CB}[7] \cdot \mathrm{C} 20$ and $\mathrm{CB}[8] \cdot \mathrm{C20}$ and the formation of a $\mathrm{CB}[8]_{3} \cdot \mathrm{C2O}_{3}$ trinitroxide supraradical both in solution and in the solid state. Similar conclusions were obtained by Turro, Ramamurthy and coworkers after investigating the interactions between $\mathrm{CB}[8]$ and 


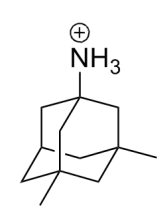

C1

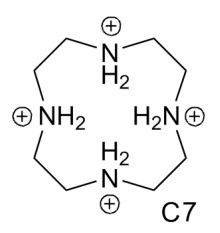

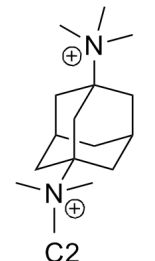

C2

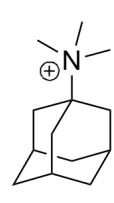

C3

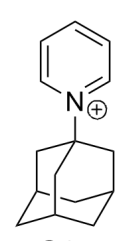

C4

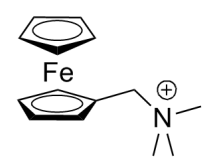

C5
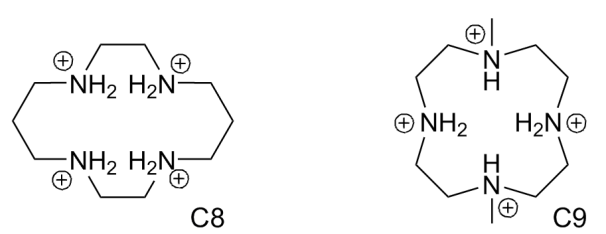

川<smiles>NCc1ccc(C[NH3+])cc1</smiles><smiles>CCC(C)(C)CC(C)C[NH3+]</smiles>

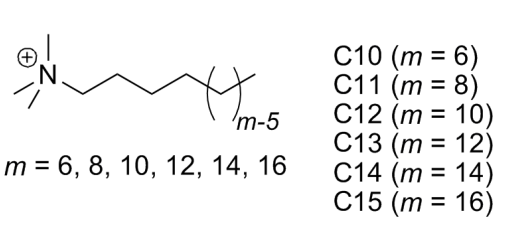<smiles>CCCC(C)(CCC[n+]1ccccc1)C[n+]1ccccc1</smiles>

$m=1,4$ C16 $(m=1)$
C17 $(m=4)$<smiles>CON1C(C)(C)CC([NH3+])CC1(C)C</smiles>

C19<smiles>COC1CC(C)(C)N(OC)C(C)(C)C1</smiles>

$\mathrm{C} 20$<smiles>CON1C(C)(C)CC([N+](C)(C)C)CC1(C)C</smiles>

$\mathrm{C} 21(m=0)$

C22 $(m=7)$

C23 $(m=11)$

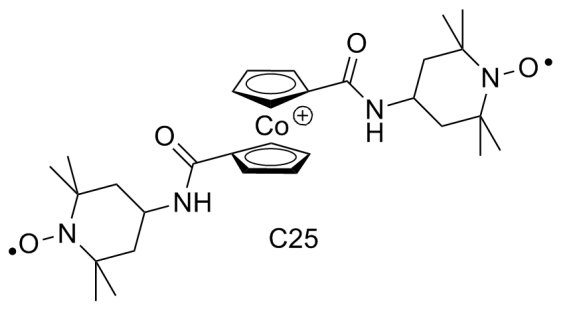

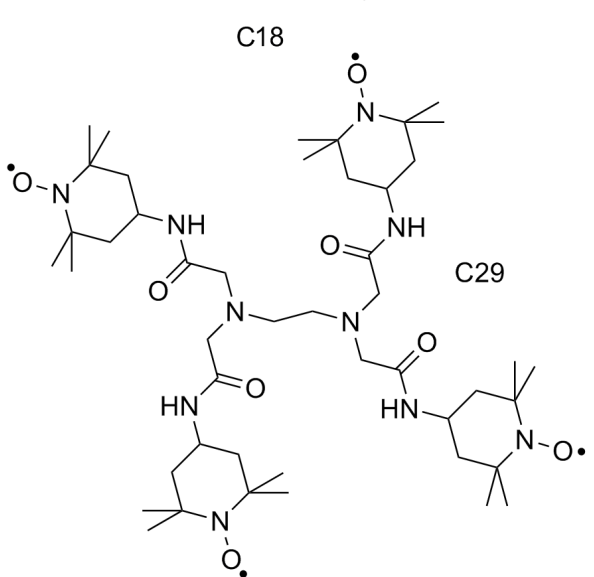

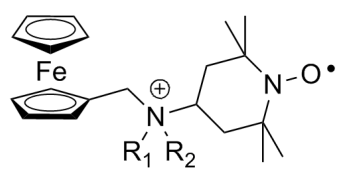

$\mathrm{C} 26 \mathrm{R}_{1}=\mathrm{R}_{2}=\mathrm{H}$

$\mathrm{C} 27 \mathrm{R}_{1}=\mathrm{H}, \mathrm{R}_{2}=\mathrm{CH}_{3}$

$\mathrm{C} 28 \mathrm{R}_{1}=\mathrm{R}_{2}=\mathrm{CH}_{3}$

Figure 11: Selected guests for CB[8]. 
nitroxides C21-C23. ${ }^{120}$ Kaifer and coworkers have investigated the interaction between $\mathrm{CB}[n]$ and a series of cobaltocenium and ferrocene derivatives incorporating nitroxide units into their structures (see C24 to C28 in Figure 11). ${ }^{121-123}$ Nitroxide derivative C24 exhibits a bimodal binding interaction with $\mathrm{CB}[7]$ and $\mathrm{CB}[8]$. While $\mathrm{CB}[7]$ prefers to bind to the cobaltocenium group, $\mathrm{CB}[8]$ interacts with the nitroxide group forming a highly stable host-guest complex (see Table 6). On the other hand, C25 contains two nitroxide radicals thus allowing the quantitative formation of a ternary $\mathrm{CB}[8]_{2} \cdot \mathrm{C} 25$ host-guest complex at micromolar concentrations. The binding of $\mathrm{CB}[8]$ imparts rigidity to the final supramolecular structure and effectively hinders any spin exchange coupling, which results in a simple three-line EPR spectrum of the complex. The ternary complex was even characterized by solid state by $\mathrm{X}$-ray diffraction. Tordo and coworkers have recently reported the reversible modulation of the spin exchange interaction in tetranitroxide C29 controlled by the allosteric complexation of $\mathrm{CB}[8] \cdot{ }^{124}$ Addition of $\mathrm{CB}[8]$ to an aqueous solution of $\mathbf{C 2 9}$ transforms the nine line spectrum of the tetranitroxide into a three line spectrum characteristic of a mononitroxide. The noncovalent interaction between $\mathrm{CB}[8]$ and C29 involves a highly cooperative asymmetric complexation $\left(\log \mathrm{K}_{1}=3.60 \mathrm{M} ; \log \mathrm{K}_{2}\right.$ $=5.30 ; \alpha=201)$ whereby two $\mathrm{CB}[8]$ molecules bind tightly to a single $\mathbf{C 2 9}$ guest leading to a rigid complex with remote nitroxide moieties. The authors attribute the high allosteric interaction energy $\left(\Delta \Delta G_{\text {allos }} \cdot \mathbf{C} 29 \approx 13 \mathrm{~kJ} \cdot \mathrm{mol}^{-1}\right)$ to a combination of different factors including the pre-organized skeleton of the multivalent guest which is multiply H-bond donating, a potential $\mathrm{CB}[8]$-induced shift of the $\mathrm{pKa}$ of the internal amines and the intrinsic conformational properties of $\mathbf{C 2 9}$. The binding of the first $\mathrm{CB}[8]$ molecule in this instance induces a large conformational change in the guest, which favors the binding of the second $\mathrm{CB}[8]$ molecule.

Although the smaller homologues of the $\mathrm{CB}[\mathrm{n}]$ family can bind only one guest inside the cavity, $\mathrm{CB}[8]$ is unique in its ability to bind two guests, as has been shown by Kim and others. ${ }^{128-132}$ This rather unique binding property has been exploited in a wide 
variety of applications including novel supramolecular polymeric materials, ${ }^{133-135}$ catalysis ${ }^{20,136-138}$ and sensing. ${ }^{98,139-141} \mathrm{CB}[8]$ promotes (hetero)dimerization of two $\pi$-systems in aqueous solution and Kim and coworkers have shown that $\mathrm{CB}[8] \cdot \mathrm{C} 30$ can bind to a variety of other aromatic guests including 2,6-dihydroxynaphthalene, 1,4-dihydroxybenzene, tyrosine, dopamine and thymine, phenylalanine, tryptophan, tryptophan derivatives and tryptophan-containing peptides. ${ }^{128,129,142}$ Based on this concept, a CB[8] heteroternary complexation strategy to the reversible PEGylation of bovine serum albumin and proteinprotein dimer formation was developed by Scherman et al. ${ }^{143}$ Brunsveld and coworkers have also exploited this concept in protein-protein binding and protein-to-surface attachment by using methylviologen and naphthalene-functionalized fluorescent proteins and $\mathrm{CB}[8]{ }^{144,145}$

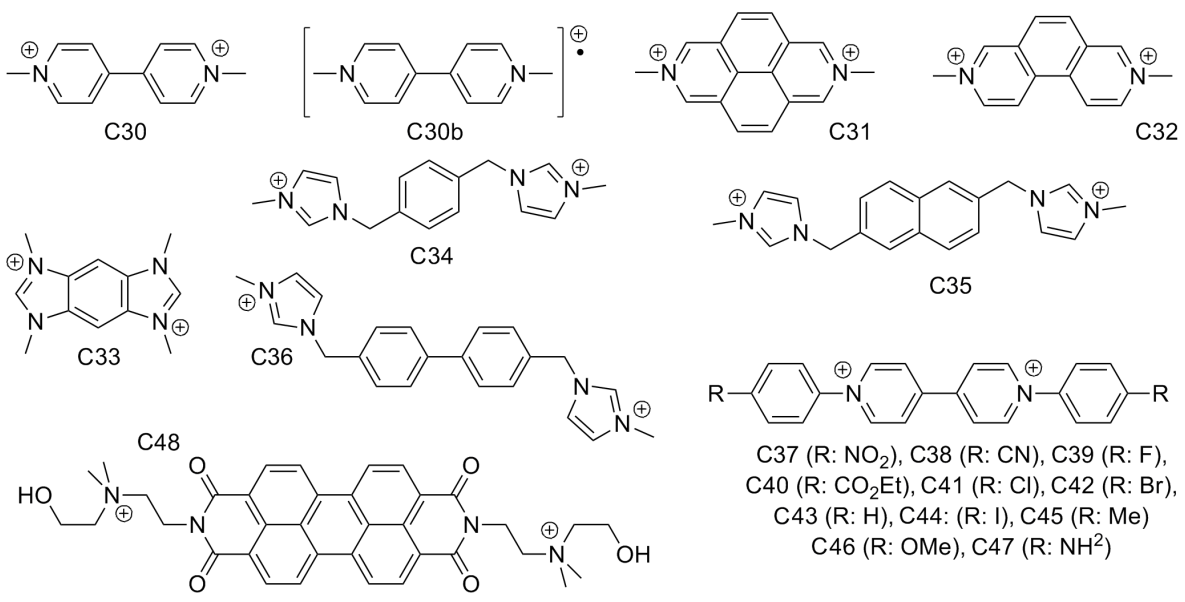

Figure 12: Selected guests for CB[8] that can act as a first guest for heteroternary complex formation.

Scherman et al. has studied in detail the equilibria of a series of ternary complexes based on $\mathrm{CB}[8] \cdot \mathrm{C} 30$ and a wide variety of aromatic second guest molecules by ITC and ESI-MS (see Table 8 and Figure 12). The binding constants were found to be between $\log K=2.00-6.00$. An excellent agreement between solution-based titrations (ITC) and gas-phase studies was found when free energies of solvation are taken into consideration. ${ }^{146}$ Kaifer and coworkers have reported the formation of $\mathrm{CB}[8]$ ternary complexes between 2,7-dimethyldiazapyrenium, as first guest, and catechol and dopamine as sec- 
ond guests. ${ }^{147}$ In an analogous fashion, 2,7-dimethyldiazaphenanthrenium can also form ternary complexes, in the presence of $\mathrm{CB}[8]$, with tryptophan and serotonin. ${ }^{132}$ Scherman and coworkers have also identify a group of doubly charged molecules as potential alternatives that increase the repertoire of first guests for $\mathrm{CB}[8]$. These include tetramethylbenzobis(imidazolium), ${ }^{148}$ aryl-bisimidazolium salts, ${ }^{149}$ aryl viologen derivatives ${ }^{42}$ and water-soluble perylene bis(diimides). ${ }^{150}$ The binding of dicationic dyes such as 2,7dimethyldiazapyrenium, as first guest to $\mathrm{CB}[8]$, has been cleverly used by Biedermann, Nau and coworkers to construct a chirality sensor and peptide-sequence recognition system at micromolar analyte concentrations. ${ }^{45,151}$

Alternatively to the previously mentioned heteroternary complexes, a number of guests have been shown to undergo double encapsulation into $\mathrm{CB}[8]$ including napththyl derivatives, ${ }^{4}$ coumarin, ${ }^{152} \mathrm{~N}$-phenylpiperazine, ${ }^{153}$ 9-aminoacridinium, ${ }^{154}$ neutral red, ${ }^{155}$ 1-[2methylnaphthyl]-3-methylimidazolium, ${ }^{156}$ berberine, ${ }^{157} p$-dimethylaminobenzonitrile, ${ }^{158}$ phenylpyridinium derivatives, ${ }^{159,160}$ bipyridinium salts, ${ }^{161}$ anthracene derivatives ${ }^{162,163}$ and some specific peptide sequences containing tryptophan or phenyalanine residues ${ }^{164,165}$

Some selected radical cations can also dimerize inside $\mathrm{CB}[8]$ in an analogous fashion to homodimer complexes. Kim and coworkers described that a high affinity $\mathrm{CB}[8]$ homoternary complex can be produced when methyl viologen is reduced to its radical cation species. ${ }^{142}$ Kaifer and coworkers have shown that $\mathrm{CB}[8]$ can be used to exert redox control over the dimerization of dendrons. For instance, dendrons containing a dicationic viologen group at their focal point can form stable 1:1 inclusion complexes with $\mathrm{CB}[8] .{ }^{166}$ Upon one electron reduction, the radical cation group of the dendronviologen species forms a dimer inside the $\mathrm{CB}$ [8] cavity, in analogy to simple viologen. This idea has been further exploited to control the structure of dimeric dendritic assemblies from viologen- and dialkoxybenzne-dendrons. ${ }^{131}$ Another interesting example of a redox $\mathrm{CB}[8]$-mediated switch consist of an equimolar mixture of methylviologen, TTF derivative $\mathrm{C} 60$ and $\mathrm{CB}[8]$. This ternary mixture results in the formation of a het- 
eroternary complex $\mathrm{CB}[8] \cdot \mathrm{MV}^{2+} \cdot \mathrm{C} 60$ potentially stabilized by electron-rich electron-poor $\pi-\pi$ stacking interactions. ${ }^{167}$ Upon treatment with $\mathrm{Na}_{2} \mathrm{~S}_{2} \mathrm{O}_{4}$, viologen is reduced to $\mathrm{MV}^{+} \bullet$, which results in complex disproportionation yielding $\mathrm{CB}[8] \cdot\left(\mathrm{MV}^{+\bullet}\right)_{2}$. Exposure to $\mathrm{O}_{2}$ returns the system to the parent heteroternary complex state. On the contrary, treatment of $\mathrm{CB}[8] \cdot \mathrm{MV}^{2+} \cdot \mathbf{C} 60$ with $\mathrm{Fe}\left(\mathrm{ClO}_{4}\right)$ oxidizes $\mathbf{C} 60$ to $\mathbf{C 6 0 b ^ { + }}$, thus resulting in a disproportionation to yield $\mathrm{CB}[8] \cdot\left(\mathbf{C} \mathbf{6} \mathbf{b}^{+\bullet}\right)_{2}$ as evidenced by $\mathrm{UV} /$ Vis spectroscopy. This last transformation can be reverted upon addition of ascorbic acid. The ternary complex $\mathrm{CB}[8] \cdot \mathrm{MV}^{2+} . \mathrm{C} 60$ can therefore be defined as a reversible molecular switch that exists in three different states in a stimulus-dependent manner.
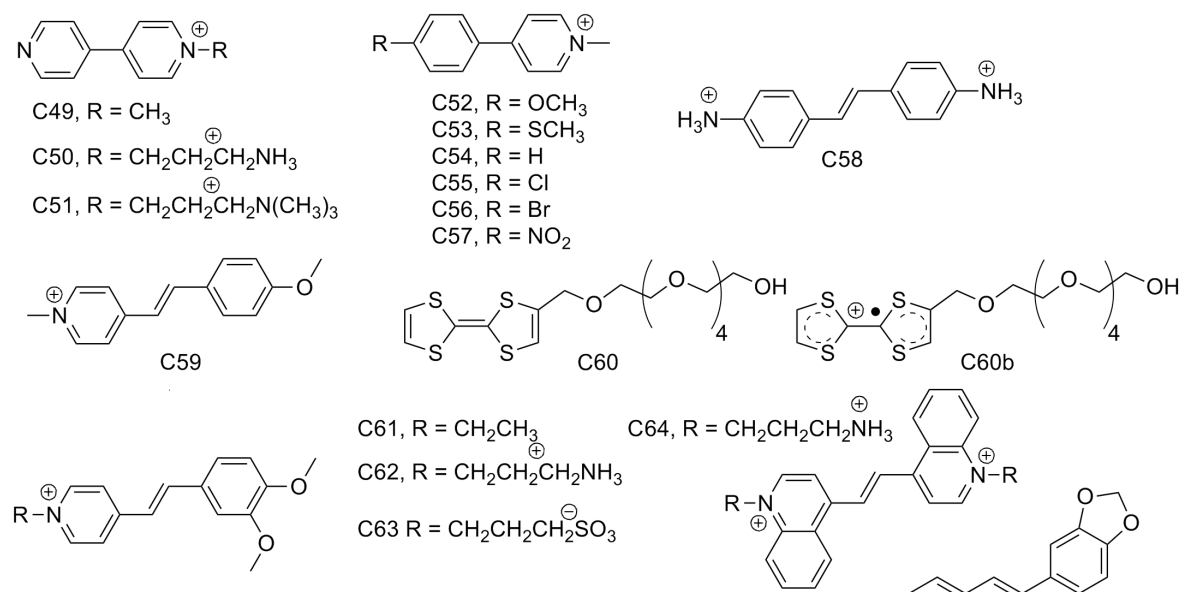

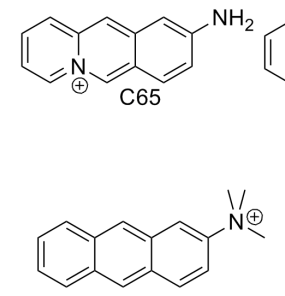

C69
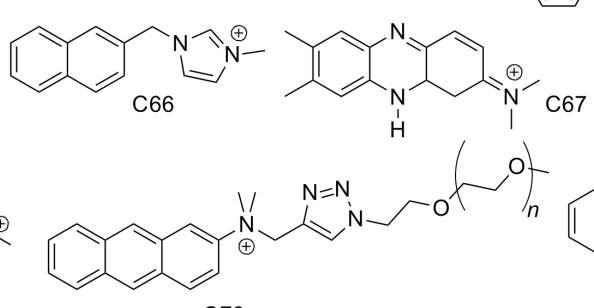

C70

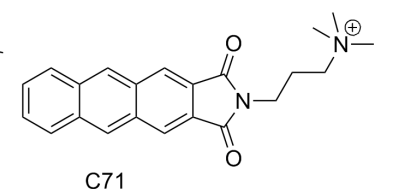

Figure 13: Selected guests for $\mathrm{CB}[8]$ that can undergo homodimerization.

\section{Encapsulation of gaseous species in $\mathrm{CB}[n]$}

Cucurbiturils have shown great promise in applications that include polymer and nanoparticle constructs because of their ability to sequester specific molecules within their unique 
inner cavities. This, in addition to cucurbiturils' high chemical and thermal stability, has driven researchers to utilize cucurbiturils in the area of gas encapsulation and storage. Sorption of gases such as $\mathrm{CO}_{2}, \mathrm{CO}$ and $\mathrm{CH}_{4}$ could lead to effective green house gas capture technologies, in addition to being incorporated into nanoparticle structures for advanced sensing and photochemical applications.

In the area of gas sorption research, metal organic frameworks (MOFs) have garnered interest. Along with porous coordination polymers (PCPs), MOFs have very high surface areas and have demonstrated selectivity and capacity for adsorbing gases like $\mathrm{CO}_{2}$ making them competitive candidates for gas encapsulation. ${ }^{168}$ The issues that MOFs and PCPs face is that they can be sensitive to water, which minimizes their utility in cleaning flue gases. Cucurbiturils, on the other hand, are stable in the presence of water and are easily made from cheap organic molecules, paving the way toward stable and effective alternatives in gas sorption. They have demonstrated gas uptake capacities comparable to some of the MOFs and PCPs. ${ }^{169,170}$

On account of the small nature of gaseous molecules, gas absorption tends to be favored by the smaller cucurbiturils. This is because the smaller inner cavities of $\mathrm{CB}[5]$ and $\mathrm{CB}[6]$ favor encapsulation of small guests as compared to the larger internal cavities of $\mathrm{CB}[7]$ and $\mathrm{CB}[8]$. Furthermore, the solubilities of $\mathrm{CB}[5]$ and $\mathrm{CB}[6]$ have allowed their application in sorption of gases in different ways, with the water soluble $\mathrm{CB}[5]$ finding use in aqueous phase gas sorption, and the less soluble $\mathrm{CB}[6]$ being favored for use in powdered form geared towards the cleaning of flue gases. However, $\mathrm{CB}[6]$ is not limited in its use in aqueous phase gas sorption by its low aqueous-solubility because its solubility can be enhanced in water through the use of alkali metal ions and water soluble guest molecules. Also, it is readily soluble in strongly acidic solutions. ${ }^{25,69}$ 


\section{1 $\mathrm{CB}[5]$ gas sorption}

A permethylated form of $\mathrm{CB}[5]$ (decamethylcucurbit[5]uril) has been shown by Miyahara and co-workers to encapsulate a variety of gases in both solid form and in aqueous media. ${ }^{171}$ It was found that gases of intermediate size could be encapsulated within the cavity of solid decamethylcucurbit[5] uril, such as $\mathrm{N}_{2}, \mathrm{O}_{2}, \mathrm{Ar}, \mathrm{N}_{2} \mathrm{O}, \mathrm{CO}$ and $\mathrm{CO}_{2}$, and readily released upon heating, with $\mathrm{N}_{2} \mathrm{O}$ and $\mathrm{CO}_{2}$ being the most readily absorbed of the gases (crystal structures shown in Figure 14). Smaller gases $\left(\mathrm{He}, \mathrm{Ne}\right.$ and $\mathrm{H}_{2}$ ) move too freely in and out of the portals to be absorbed, and larger gases $\left(\mathrm{Kr}, \mathrm{Xe}\right.$, and $\left.\mathrm{CH}_{4}\right)$ could also not be encapsulated. In the aqueous phase, however, all of the aforementioned gases could be absorbed, with the larger gases requiring heating to $80^{\circ} \mathrm{C}$ to be incorporated. This suggests that the solvent molecules play a role in encouraging the encapsulation of the larger gases, as it is unlikely that the portal size of the $\mathrm{CB}[5]$ would increase significantly upon heating as a result of the rigidity of the macrocycle. ${ }^{171}$

\section{2 $\mathrm{CB}[6]$ gas sorption}

Solid $\mathrm{CB}[6]$ has been shown to have an affinity for $\mathrm{CO}_{2}$ encapsulation, with a sorption capacity that is comparable to that of MOFs, including HKUST- 1 and $\left[\mathrm{Pd}\left(2-\right.\right.$ pymo)2]n. ${ }^{170} \mathrm{In}$ 2010, Kim et al. studied the $\mathrm{CO}_{2}$ sorption properties of solid CB[6], precipitated from $\mathrm{HCl}$ with a honeycomb like crystal structure. Gas absorption isotherms for $\mathrm{CO}_{2}, \mathrm{CH}_{4}$ and $\mathrm{CO}$ showed a high capacity for $\mathrm{CO}_{2}$ storage, and selectivity for $\mathrm{CO}_{2}$ over both $\mathrm{CO}$ and $\mathrm{CH}_{4}$, with $45 \mathrm{~cm}^{3} \mathrm{~g}^{-1}$ absorbed at $298 \mathrm{~K}$ (1 bar). This could be increased to $97 \mathrm{~cm}^{3} / \mathrm{g}^{-1}$ at $196 \mathrm{~K}$ (1 bar). X-ray analysis of $\mathrm{CB}[6]$ after $\mathrm{CO}_{2}$ sorption demonstrated that $\mathrm{CO}_{2}$ was not only bound to the inner cavity of the cucurbiturils, but also externally to the CB molecules via hydrogen bonding and dipole-quadrupole interactions with the carbonyl portals (15(a)). This demonstrated that the selectivity of solid $\mathrm{CB}[6]$ towards $\mathrm{CO}_{2}$ could be useful in removing $\mathrm{CO}_{2}$ from flue gases. 

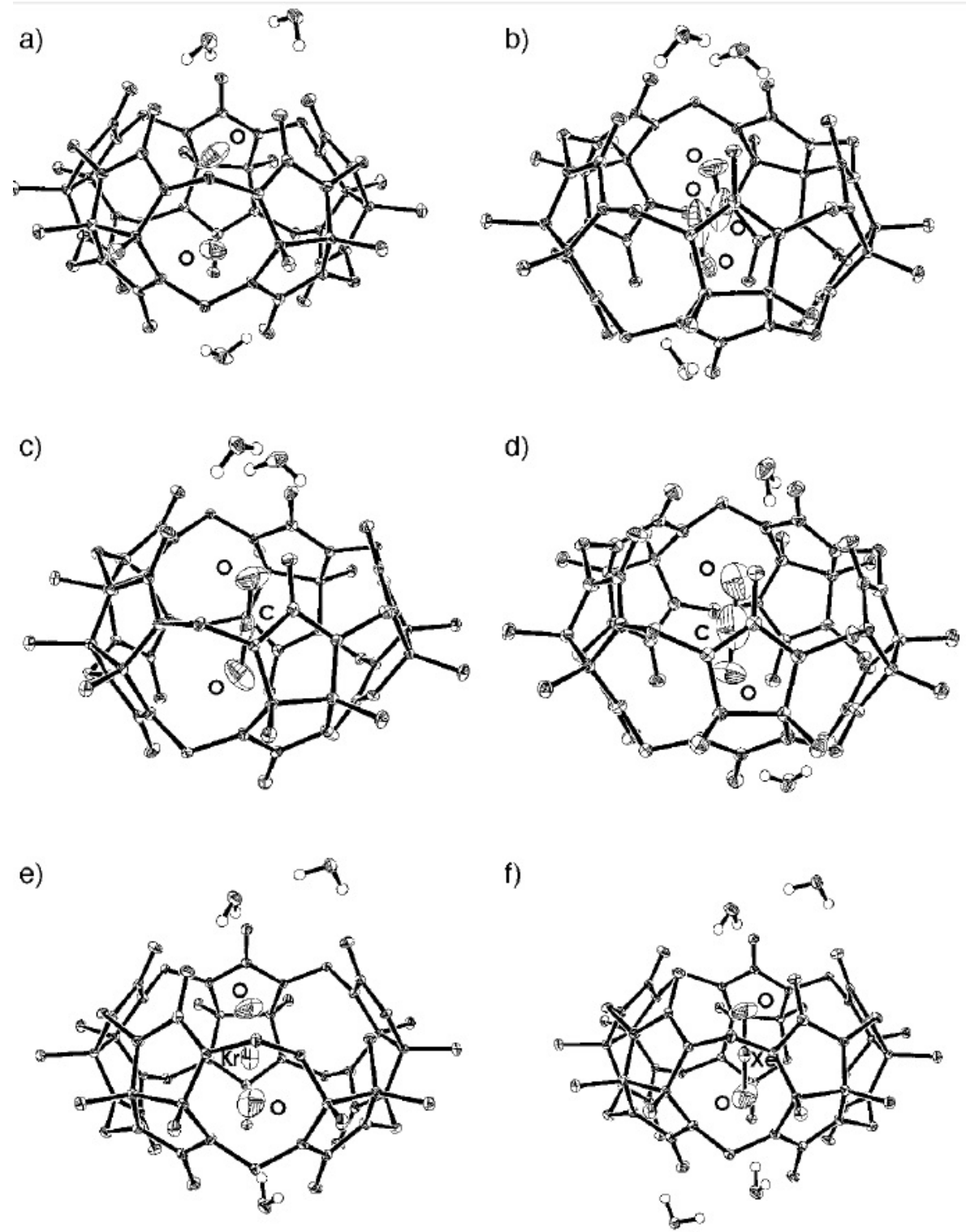

Figure 14: X-ray crystal structures of decamethylcucurbit[5]uril-gas complexes: a) $\mathrm{He}$ complex, b) $\mathrm{O}_{2}$ complex, c) $\mathrm{CO}_{2}$ complex, d) $\mathrm{CH}_{4}$ complex, e) $\mathrm{Kr}$ complex, f) Xe complex. Reproduced from Miyahara et al. Angew. Chem. Int. Ed. 2002, 41, 3020-3023 ${ }^{171}$ with permission from John Wiley and Sons, Inc. 
Continuing on from the work of Kim et al., Tian and co-workers successfully synthesized three alternate crystalline forms of $\mathrm{CB}[6]$, termed forms I, II and III, via the mixing of ethanol into a solution of $\mathrm{CB}[6]$ in $6 \mathrm{M} \mathrm{HCl} .{ }^{172}$ Form I crystals were shown to be in the monoclinic point group, with the $\mathrm{CB}[6]$ molecules stacking into a double bilayer structure (Figure15(b)). Form III crystals were shown to be rhombohedral in nature, with the molecules stacking in a bilayer structure Figure (15(c)), whilst form II crystals were unable to be characterized by X-ray crystallography owing to difficulties in growing suitable crystals for diffraction. Despite this, form II exhibited the best thermal stability, with forms I and III losing crystallinity upon heating, as well as the highest surface area, with BET isotherms yielding values of $276 \mathrm{~m}^{2} \mathrm{~g}^{-1}$. The high surface area and thermal stability of form II gives the material promise in gas storage applications, with $76 \mathrm{~cm}^{3} \mathrm{~g}^{-1}$ of $\mathrm{CO}_{2}$ uptake at $298 \mathrm{~K}$ (1 bar). Although more focused on catalytic applications, Reddy et al. demonstrated that solid $\mathrm{CB}[6]$ has a propensity to absorb $\mathrm{I}_{2}$ and $\mathrm{Br}_{2}$ vapor, leading to distinctly colored CB[6]-halogen powders. ${ }^{81}$ The catalytic properties of these materials shall be discussed in Section 11.

Whilst pure $\mathrm{CB}[6]$ is generally not suitable for gas encapsulation in aqueous environments because of its inherently low solubility, it can be made soluble through the use of metal ions, aliphatic or aromatic ammonium ions, or neutral saline aqueous solutions. In such environments, $\mathrm{CB}[6]$ has been demonstrated to encapsulate $\mathrm{Xe}^{173,174} \mathrm{SF}_{6}{ }^{175,176}$ as well as gaseous alkanes and alkenes. ${ }^{69}$

El Haouaj et al. demonstrated the encapsulation of Xe by $\mathrm{CB}[6]$ in a $0.2 \mathrm{M}$ solution of $\mathrm{Na}_{2} \mathrm{SO}_{4}$, with binding constants ranging from $2.1 \times 10^{2} \mathrm{M}^{-1}$ (although $3.4 \times 10^{2} \mathrm{M}^{-1}$ has been reported for an alkylated $\mathrm{CB}[6]$ with higher water solubility ${ }^{177}$ ). The reversible binding was confirmed through ${ }^{1} \mathrm{H}$ and ${ }^{129} \mathrm{Xe} \mathrm{NMR}$, and it was shown that $\mathrm{pH}$ played little role in the sorption of the noble gas. $\mathrm{SF}_{6}$ has also been shown to be encapsulated in aqueous $\mathrm{CB}[6]$, however binding constants vary. ${ }^{175,176}$ The first report of $\mathrm{SF}_{6}$ binding, confirmed via ${ }^{19} \mathrm{~F} \mathrm{NMR}$, yielded a binding of $3.1 \times 10^{4} \mathrm{M}^{-1}$ in $0.2 \mathrm{M} \mathrm{Na}_{2} \mathrm{SO}_{4}$, however, 


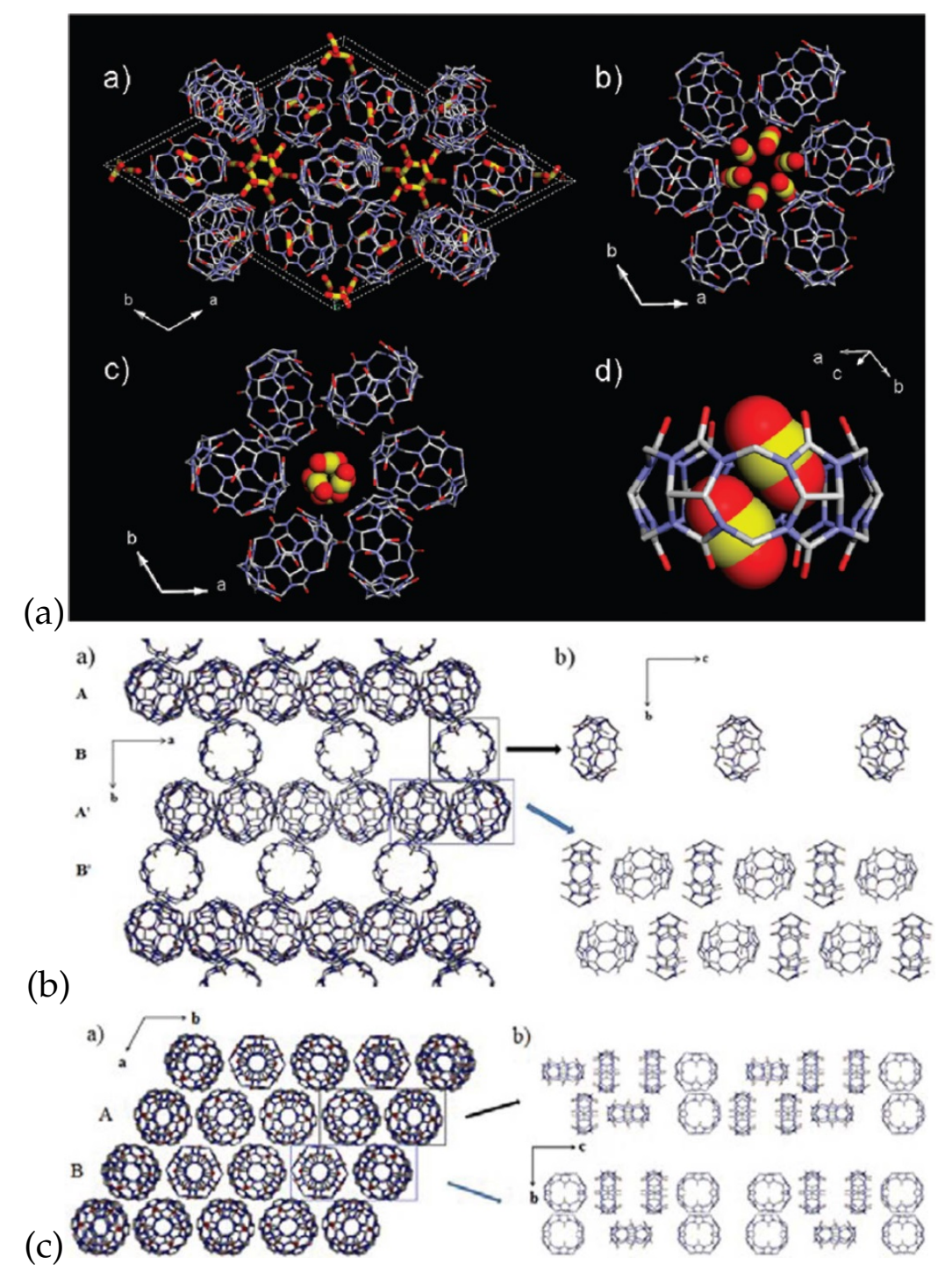

Figure 15: (a)X-ray crystal structures of $\mathrm{CB}[6]-\mathrm{CO}_{2}$ binding at different sorption sites. (b) Crystal structure of $\mathrm{CB}$ [6] arrange in the Form I lattice. (c) Crystal structure of $\mathrm{CB}[5]$ arranged in the Form III lattice. (a) Reprinted with permission from Kim et al. J. Am. Chem. Soc. 2010 132, 12200. ${ }^{170}$ Copyright 2010 American Chemical Society. (b) and (c) reproduced from Tian et al. CrystEngComm 2013 15, 1528-1531 172 with permission from The Royal Society of Chemistry. 
binding in pure water yields a binding constant of $6.6 \times 10^{5} \mathrm{M}^{-1} .176$ The higher binding constant in pure water could be attributed to the absence of cations in the solution, which would potentially block the carbonyl portals of the $\mathrm{CB}[6]$ and perturb gas encapsulation.

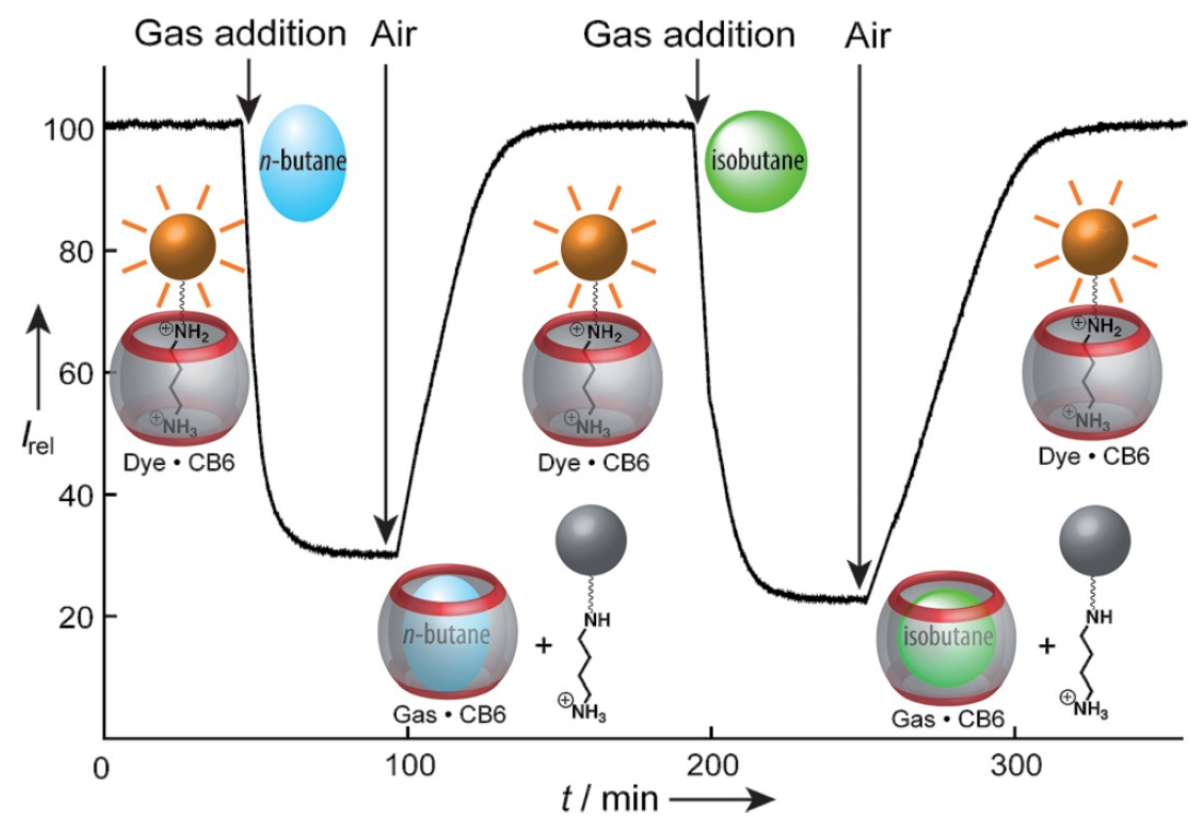

Figure 16: Fluorescent dye displacement method for gas sensing in aquesous solutions of CB[6]. Reproduced from Florea et al. Angew. Chem. Int. Ed. 2011 50, 9338-9342 ${ }^{69}$ with permission from John Wiley and Sons, Inc.

The difficulty of determining binding constants for gases in aqueous CB[6] systems arises from the low solubility of $\mathrm{CB}[6]$ in water. 5 To overcome this, Florea et al. adopted a dye displacement titration method for measuring the binding constants of hydrocarbon gases (see Figure 16 for schematic). ${ }^{69}$ A compound with a putrescine moiety for binding to $\mathrm{CB}[6]$, tethered to a 1-naphtylamine-5-sulphonic acid chromophore was used as the displacement dye because of the enhancement in its fluorescence upon binding to $\mathrm{CB}[6]$ and its ability to dramatically increase the solubility of $\mathrm{CB}[6]$ in water. The fluorescence of the dye was monitored as gases were introduced into the aqueous system and binding constants were calculated from the final fluorescence intensity, assuming 1:1 stoichiometric binding. Binding constants ranged from $<2 \mathrm{M}^{-1}$ for methane and neopentane up to $1300 \pm 300 \mathrm{M}^{-1}$ for cyclopentane (binding constants from the cited work can be found in 
Table 3).

\subsection{Gas sorption in higher $\mathrm{CB}[n]$ homologues}

To date there has only been one report on gas sorption in $\mathrm{CB}[7]$, which has shown a high sorption capacity for various gases, including $\mathrm{CO}_{2}, \mathrm{SO}_{2}$, and $\mathrm{H}_{2} \mathrm{~S} .{ }^{169}$ Of the three gases, $\mathrm{SO}_{2}$ showed the highest capacity for being absorbed, with uptake as high as $105 \mathrm{~cm}^{3} \mathrm{~g}^{-1}$, followed by $\mathrm{H}_{2} \mathrm{~S}\left(62.5 \mathrm{~cm}^{3} \mathrm{~g}^{-1}\right)$ and $\mathrm{CO}_{2}\left(50 \mathrm{~cm}^{3} \mathrm{~g}^{-1}\right)$. Although $\mathrm{CH}_{4}$ and $\mathrm{N}_{2}$ were tested, low uptakes of $5.5-6 \mathrm{~cm}^{3} \mathrm{~g}^{-1}$ were observed. Reports of gas absorption in CB[8] and $\mathrm{CB}[10]$ have not yet appeared. 


\section{Cucurbit $[n]$ urils on surfaces}

The influence of the overall electrostatic potential map of $\mathrm{CB}[n]$ on its molecular encapsulation properties is evident. The high electron density at its carbonyl portals, however, governs the external interactions of $\mathrm{CB}[n]$ with materials such as cations and metallic surfaces (Figure 17a). The latter property is increasingly drawing attention for applications ranging from nanoparticle synthesis, assembly, catalysis and plasmon-assisted molecular sensing. In addition, $\mathrm{CB}[n]$ can also be localized at interfaces through interactions with immobilized guests on surfaces (Figure 17b), as well as by covalently linking functionalized homologues of $\mathrm{CB}[n]$ directly to a surface (Figure 17c). Although electrostatic interactions of $\mathrm{CB}[n]$ are mainly limited to metallic substrates, the other two types of binding strategies are applicable to other substrates such as silica and quantum dots. These interactions have been applied successfully to design novel sensors and reversible architectures. Progress in these fields will be discussed in this section.
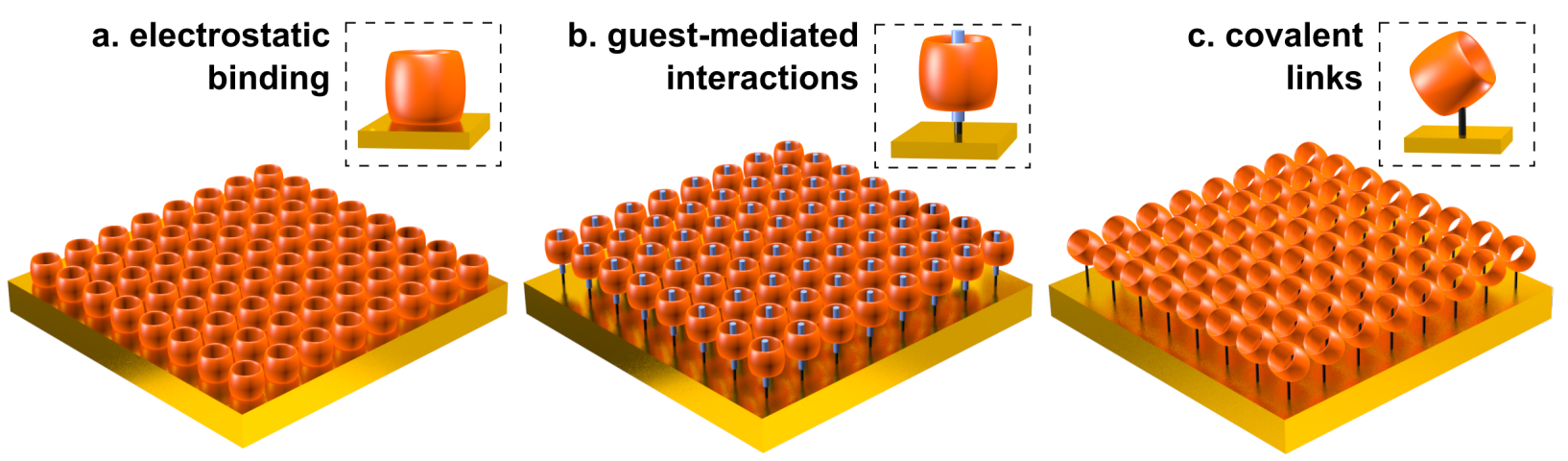

Figure 17: Strategies used for immobilizing $\mathrm{CB}[n]$ on various substrates: (a) carbonyl portal mediated binding electrostatic interactions (b) threading of $\mathrm{CB}[n]$ through guests tethered on the surface and (c) direct linking using functionalized homologues of $\mathrm{CB}[n]$.

\subsection{Electrostatic interactions}

The direct supramolecular interaction of $\mathrm{CB}[n]$ with gold was first successfully characterized by An Q. et al. in 2008, ${ }^{178}$ where the interaction between gold nanoparticle colloids and $\mathrm{CB}[n]$ was established using TEM and UV-vis characterization. $\mathrm{CB}[n]$ acted as a 'glue' 
between adjacent nanoparticles, resulting in the formation of coagulates (Figure 18a,b). In a further step, the formation of CB[7] self-assembled monolayers was achieved simply by dipping a planar gold substrate into a saturated aqueous solution of $\mathrm{CB}[7]$. The $\mathrm{CB}[7]$ molecules physisorb on the gold surface. Surface characterization techniques, including Fourier transform IR reflection absorption spectroscopy and XPS indicated that the interactions between $\mathrm{CB}[7]$ and gold occurred through the electronegative carbonyl portals of $\mathrm{CB}[7]$, where the $\mathrm{CB}[7]$ portals were oriented in a perpendicular fashion with respect to the gold surface (Figure 18c). In the same study, the surface packing of $\mathrm{CB}[7]$ was estimated to be $>48 \%$ using an indirect electrochemical method, nevertheless, the true density of $\mathrm{CB}[n]$ molecules on flat metallic surfaces remain unascertained.

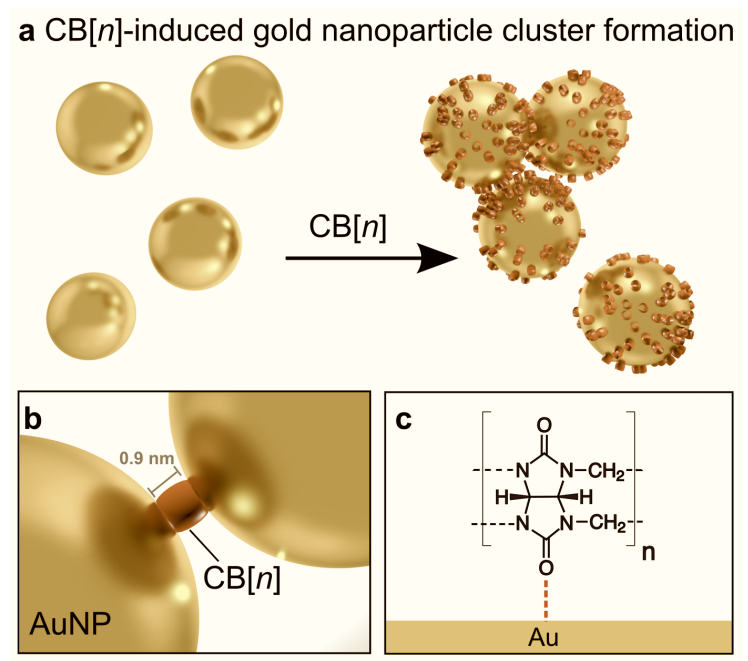

Figure 18: (a) $\mathrm{CB}[n]$ acts as a 'glue' in between gold nanoparticles forming nanoparticle coagulates, (b) the nanoparticles are held apart by $\mathrm{CB}[n]$ molecules at a precise distance of $0.9 \mathrm{~nm}$, (c) $\mathrm{CB}[n] \mathrm{s}$ bind to metallic surfaces through its carbonyl portals and are oriented perpendicular with respect to the gold surface.

Modification and assembly of nanoparticles with $\mathrm{CB}[n]$ : Despite the limited fundamental knowledge about $\mathrm{CB}[n]$ interactions with metallic surfaces, particularly in colloidal systems, $\mathrm{CB}[n]$ has been shown to be useful in the synthesis of nanoparticles, mainly as a 'capping agent' or 'stabilizing ligand'. The first synthesis of $\mathrm{CB}[n]$-assisted nanoparticles was reported by Geckeler et al., where $\mathrm{CuO}$ nanoparticles (diameter $\backsim 5 \mathrm{~nm}$ ) were formed using precursor-encapsulated $\mathrm{CB}[7] \cdot{ }^{179}$ The same group later reported a 
one-pot synthesis of silver ${ }^{180}$ and gold nanoparticles ${ }^{181}$ by the reaction of aqueous $\mathrm{CB}[7]$ solutions, containing the respective precursor salts and $\mathrm{NaOH}$, at room temperature. In both cases, $\mathrm{CB}[7]$ acted as a stabilizing ligand and generated well-dispersed nanoparticles. Since no additional reducing agents were used, the role of $\mathrm{CB}[7]$ as a reducing agent, in addition to stabilizing ligand, in nanoparticle synthesis was also established. However, it should be noted that the oxidized organic species resulting from the salt reduction remains unidentified. The same applies to the reaction mechanism.

In the same year, Scherman et al. reported the formation of dynamic aggregates of gold nanoparticles using $\mathrm{CB}[5] .{ }^{182}$ In this case, the nanoparticles were prepared in the presence of a reducing agent as well as CB[5], where concurrent with other reports, ${ }^{180,181}$ $\mathrm{CB}[5]$ was once again shown to act as a capping agent. The sizes of these aggregates could be controlled by varying the ratio between $\mathrm{CB}[5]$ and the gold precursor and was based on the interactions of one or both carbonyl portals of the $\mathrm{CB}[5]$ molecules. The symmetric structure of the $\mathrm{CB}[n]$ is of particular importance to allow the $\mathrm{CB}[5]$ molecules to bridge two nanoparticles. The aggregation process was monitored by measuring the changes in the surface plasmon resonance of the colloidal solution. Typically, monodisperse gold nanoparticles absorb in the green region of the visible spectrum and appear as red solutions. Upon aggregation, the absorbance signal undergoes a red-shift and the solutions appear purple or blue instead. The kinetic evolution of these plasmonic modes were reported later in greater detail. ${ }^{183}$

This was followed by another study, where $\mathrm{CB}[5], \mathrm{CB}[7]$ as well as the less water soluble homologues, $\mathrm{CB}[6]$ and $\mathrm{CB}[8]$, were also investigated as capping agents using metastable gold nanoparticles. ${ }^{184}$ Importantly, the interparticle spacing between neighboring nanoparticles was confirmed by TEM to be approximately $1 \mathrm{~nm}$, which is consistent with the height of $\mathrm{CB}[n]$ molecules. This further indicates the portal-to-metal interactions between $\mathrm{CB}[n]$ and gold. The longer stability ( $\sim 3$ months) of the resulting nanoparticles was further attributed to the presence of sodium atoms bound to the car- 
bonyl portals of the $\mathrm{CB}[5]$ ligands. Once again, the aggregates exhibited reversible control as before.

More recently, CB[7]-stabilized gold nanoparticles were synthesized in order to demonstrate the increase in catalytic efficiency of nanoparticles free from organic ligands and metal cations. ${ }^{185}$ The stabilization of silver nanoparticles with $\mathrm{CB}[n]$ homologues have also been further investigated, ${ }^{186}$ whereby computational studies supported the assumption that the stabilizing effect of $\mathrm{CB}[7]$ is a result of lateral interactions between the oxygen atoms at the $\mathrm{CB}[7]$ carbonylated rim and the metal surface.

It is noteworthy, however, that the role of $\mathrm{CB}[n]$ capped gold nanoparticles is not restricted to stabilization of nanoparticles. Plasmonic coupling between aggregated nanoparticles affects their optical response and leads to strongly enhanced electric fields in the gaps between them. ${ }^{187,188}$ The magnitude of those fields increases near-exponentially as the distance between the particles is reduced and is limited only by electron tunneling for gaps below $\sim 0.5 \mathrm{~nm} .{ }^{189-193}$ Owing to the symmetrical geometry of $\mathrm{CB}[n]$, the presence of $\mathrm{CB}[n]$ in between nanoparticles ensures that they are held precisely $0.9 \mathrm{~nm}$ apart from each other (Figure 18c). ${ }^{183}$ This provides both extremely high and reproducible local field enhancements in the gap junctions ${ }^{194,195}$ while maintaining an accessible gap for small molecules. ${ }^{139,141}$ Since such precision at these scales is not easily achievable, physicists have to predominantly rely on theoretical simulations in order to understand the optics at such nanoscale regimes. Therefore, $\mathrm{CB}[n]$ provides a support handle to experimentally investigate the plasmon modes and near-field properties of nanoparticle clusters with well-defined separations. ${ }^{196}$

The precise gap distances between adjacent nanoparticles produced by $\mathrm{CB}[n]$ has further useful implications. For example, these $\mathrm{CB}[n]$-gold nanoparticle hybrids have been utilized to create near-identical, continuous strings of nanoparticles joined by a conducting gold thread. ${ }^{197}$ In this study, precise control over the formation of the nanomaterial was achieved on a large scale by irradiating the $\mathrm{CB}[n]$-mediated nanoparticle assem- 
blies with a suitable femtosecond pulsed laser. As a result of high field intensities in the gap-junctions between the nanoparticles, the surface atoms are drawn into the gap through optical forces and non-thermal melting and form solid metal threads between the particles. The extreme enhanced fields in the crevices of the resulting threads open up prospects for applications of these metamaterials in photovoltaics and sensing probe construction.

In consideration of the interesting plasmonic and electronic properties afforded by $\mathrm{CB}[n]$-mediated gold nanoparticle assemblies, sophisticated strategies have been developed in order to control the morphologies of these clusters. One such strategy allows the well-defined growth of one-dimensional chains of gold nanospheres, spaced by $\mathrm{CB}[n]$ molecules. ${ }^{198}$ This is achieved in a chamber consisting of gold nanoparticles and $\mathrm{CB}[n]$ solution separated by a nanoporous polycarbonate membrane. The one-dimensional chains are then formed at the membrane interface, by the electrophoretic flow of the nanoparticles under an applied voltage and the electroosmotic flow of the $\mathrm{CB}[n]$ (Figure 19).

End-to-end gold nanorod alignments can also be formed through $\mathrm{CB}[n]$ linkers. ${ }^{199}$ Centrifugation of CTAB-coated gold nanorod solutions preferentially destabilizes the CTAB bilayer at the curved $\{111\}$ end facets of the nanorods. Therefore, upon subsequent addition, $\mathrm{CB}[5]$ is only able to interact with the nanorod ends, consequently forming nanorod chain-like topologies. The surface plasmon resonance bands of gold nanorods can be tuned over a wider range than those of non-anisotropic analogues, making them interesting candidates for plasmonic applications.

SERS Sensing An important property of $\mathrm{CB}[n]$ interactions with metallic surfaces is its ability to retain its molecular recognition properties. In other words, $\mathrm{CB}[n]$ can facilitate guest molecules from the bulk solution to approach the metal-liquid interface by acting as a 'receptor' on the surface. Surface-dependent phenomena, such as catalysis and surface-enhanced spectroscopic (SERS) techniques, generally require molecules to be localized near the surfaces and often rely on the innate affinity of the molecules to- 

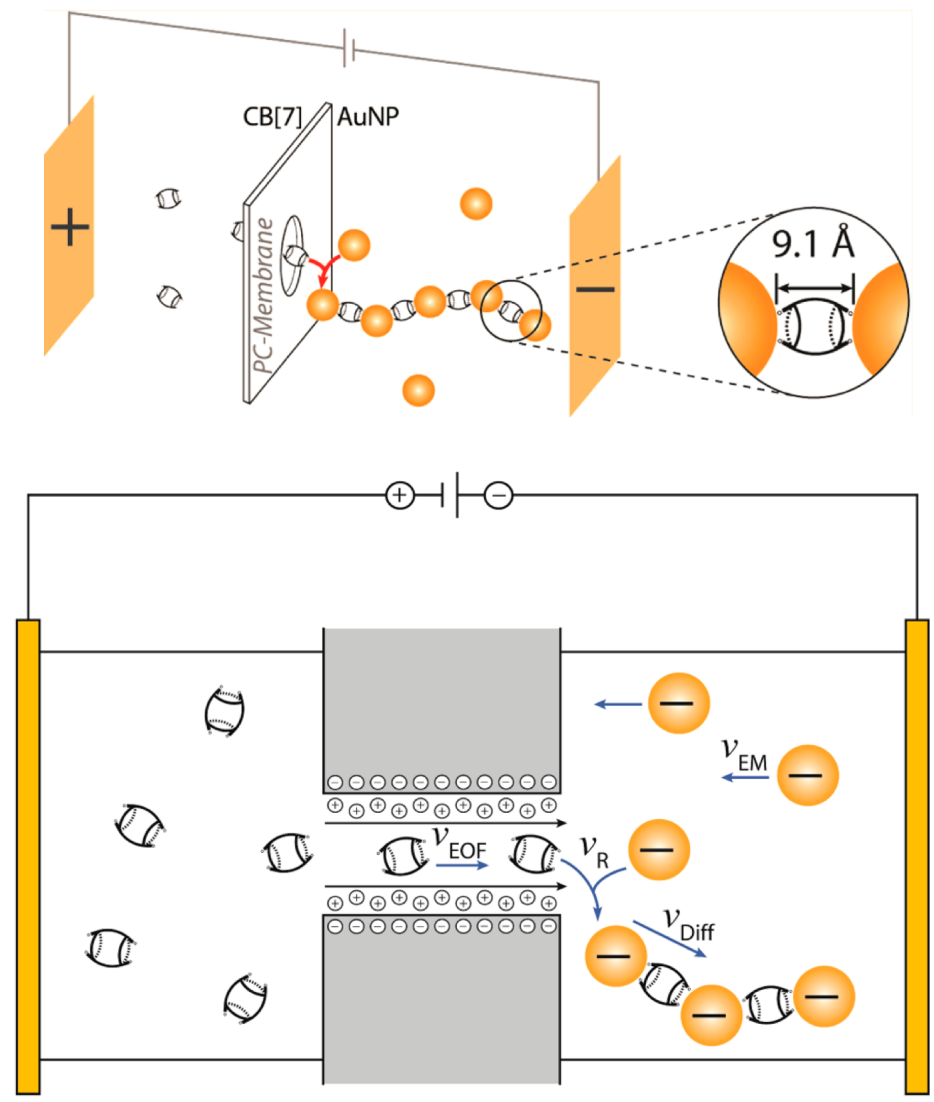

Figure 19: (a) Formation of one-dimensional chains of gold nanoparticles using an electrophoresis cell, where a nanoporous polycarbonate membrane separates two compartments containing gold nanoparticles and CB[7] respectively. (b) Nanoparticle chains are formed through synergistic electroosmotic flow of CB[7] and electrophoretic motion of the gold nanoparticles. Reprinted with permission from Hüsken et al. Nano Lett. 2013 13, 6016-6022. ${ }^{198}$ Copyright 2013 American Chemical Society. 
wards these surfaces. Since not all molecules of interest interact with surfaces spontaneously, covalent functionalization strategies are often used to immobilize them on surfaces. However, such strategies usually reduce the direct interactions of the molecules with the surface, thereby interfering with the efficiency of the surface processes. Therefore, it is evident that the ability of $\mathrm{CB}[n]$ to confine molecules of interest near the surface has dramatic significance in surface-dependent mechanisms.

The molecular recognition properties of $\mathrm{CB}[n]$ can be harnessed for sensing with SERS. ${ }^{98,139-141,183,20}$ In brief, Raman spectroscopy is a vibrational technique, which probes the inelastic scattering of photons with molecules and is able to provide a unique fingerprint for each analyte. While this method is comparable to infrared spectroscopy, the lack of interference from water molecules (in the fingerprint region) makes this method desirable for probing chemical structures, particularly in aqueous environments. However, the inherent weakness of Raman signals requires the use of enhancement mechanisms, such as the use of plasmonic surfaces, to improve sensitivity. One particularly popular approach is surface-enhanced Raman spectroscopy and the reader is referred to literature on this topic for further details. ${ }^{201-204}$

Although Raman spectroscopy was used as an analytical method to characterize encapsulation of a polymer in multiple $\mathrm{CB}[n]$ units, ${ }^{205}$ the first report of a detailed study of the Raman and SERS signature of $\mathrm{CB}[n]$ appeared in the literature $2010 .{ }^{206}$ Clearly identifiable peaks for $\mathrm{CB}[n]$ are observed in the fingerprint region $\left(400 \mathrm{~cm}^{-1}\right.$ to $\left.2000 \mathrm{~cm}^{-1}\right)$ of the Raman spectra (Figure 20). Two characteristic intense signals at approximately $450 \mathrm{~cm}^{-1}$ and $830 \mathrm{~cm}^{-1}$ are attributed to complex ring breathing modes of the $\mathrm{CB}[n]$ molecules. Interestingly, these signals show slight red and blue shifts, respectively, with an increase in the ring sizes of the different $\mathrm{CB}[n]$ homologues. The same signals and a similar trend in peak shifts is observed in the corresponding SERS spectra. A SERS enhacement factor of $10^{7}-10^{9}$ is achieved with $\mathrm{CB}[n]$ :gold nanoparticle aggregates. ${ }^{183,200}$

SERS-based sensing of a dye molecule, Rhodamine 6G (Rh6G), with the aforemen- 


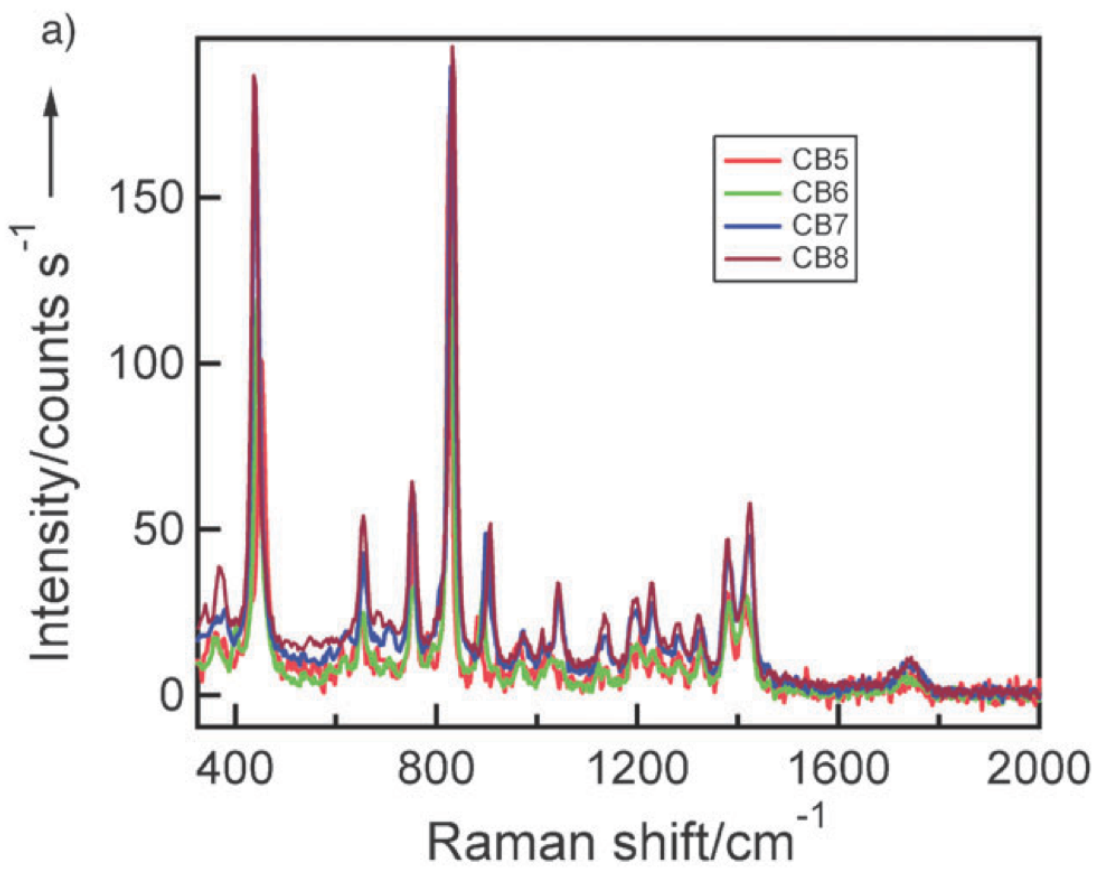

Figure 20: Raman spectra of $\mathrm{CB}[n]$ showing characteristic signals between $400 \mathrm{~cm}^{-1}$ to $2000 \mathrm{~cm}^{-1}$. Reproduced from Mahajan et al. Phys. Chem. Chem. Phys. 2010, 12, 10429-10433. ${ }^{206}$ with permission from The Royal Society of Chemistry.

tioned CB[7]:gold nanoparticle constructs was first demonstrated by Scherman et al. in 2011. ${ }^{183} \mathrm{CB}[7]$ binds to Rh6G in a 1:1 stoichiometric ratio $\left(\mathrm{K}_{a}>5 \times 10^{4} \mathrm{M}^{-1}\right) \cdot{ }^{207}$ Besides acting as an aggregating agent to glue together adjacent gold nanoparticles, $\mathrm{CB}[n]$ positions the guest molecules precisely at the point of closest approach between nanoparticles, which is where the highest field enhancement occurs, also known as a'hot-spot'. This role of $\mathrm{CB}[n]$ is particularly significant because by bringing the analyte in the hot-spot, even molecules with low Raman cross-sections undergo tremendous enhancement. For example, another SERS-study used neutral ferrocene with CB[7] (Figure 21). ${ }^{200}$ In addition, ferrocene does not have an affinity for gold surfaces but can be localized in the hot-spot using $\mathrm{CB}$ [7]. In this case, a three-step approach was used to prepare the SERS substrates, where firstly, gold nanoparticles were assembled on amino-functionalized glass. Ferrocene encapsulated CB[7] was then physisorbed on the substrate, followed by another layer of $\mathrm{Au}$ nanoparticles. As a result, gold nanoparticle clusters, predominantly dimers and trimers, were obtained on the glass surface with ferrocene encapsulated $C B[7]$ in the hot-spot re- 
gions.

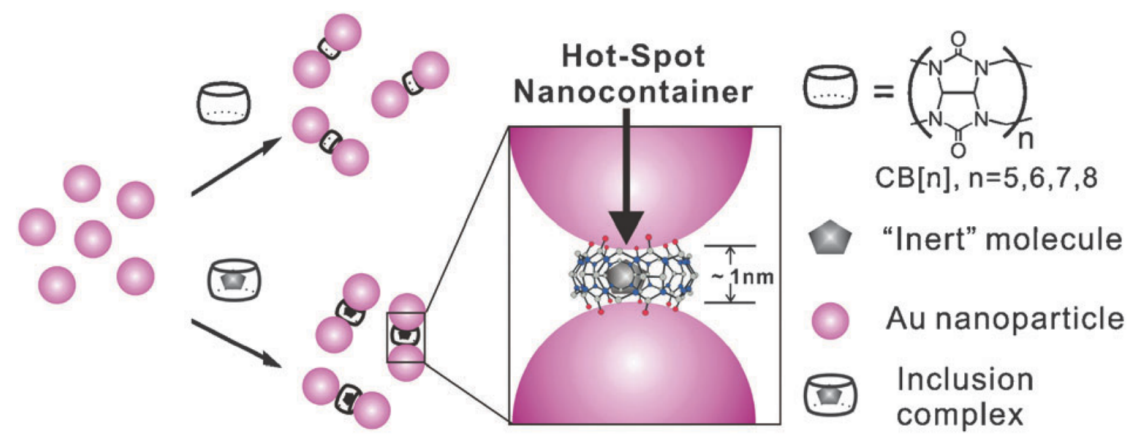

Figure 21: Formation of uniform hot-spots and localization of analytes in them for efficient surfaceenhanced Raman spectroscopy through aggregation of gold nanoparticles with $\mathrm{CB}[n]$. Reproduced from Tao et al. Chem. Commun. 2011 47, 9867-9869 ${ }^{200}$ with permission from The Royal Society of Chemistry.

The utility of this SERS based sensor has been extended to studying $\mathrm{CB}[n]$ host-guest chemistry. Computational studies had shown that $\mathrm{CB}[n]$, particularly $\mathrm{CB}[7]$, is expected to undergo a subtle structural distortion on encapsulation of a guest. ${ }^{208}$ These structural changes are evident in the SERS specta of $\mathrm{CB}[n]$ complexes and are observed in the SERS spectra as slightly shifted peaks. ${ }^{139}$ These structural changes are not as apparent in the solid-state Raman spectra of the complexes. These changes, however, are more pronounced in the Raman signals of the guest molecules. ${ }^{209}$ Not only did these results present experimental evidence to support the outcomes of the theoretical predictions, the shifted and unshifted signals also provided a tool to be able to distinguish the amounts of complexed and uncomplexed $\mathrm{CB}[n]$ s. Therefore, the system could be used for characterizing $\mathrm{CB}[n]$ host-guest binding parameters as well as absolute quantification of guest analytes, such as polyaromatic hydrocarbons. ${ }^{139}$ Roldán et al. reported the use of this strategy, using silver nanoparticles, for the detection of $\mathrm{pH}$ dependent changes in the complexation behavior of diquat with $\mathrm{CB}[8] .{ }^{140}$ The system has also been used to monitor the real-time photodimerzation of diaminostilbene inside the $\mathrm{CB}[n]$ cavity in situ. ${ }^{141}$

One of the advantages of the SERS technique is its ability to resolve analytes in a mixture, which is particularly advantageous for structurally similar molecules. $\mathrm{CB}[n]$ compliments this property of SERS by acting as a receptor for several molecular analogues. 
A recent report used this property to test the multiplexing abilities of $\mathrm{CB}[n]$ in sensing structurally similar metabolites in urine. ${ }^{98}$

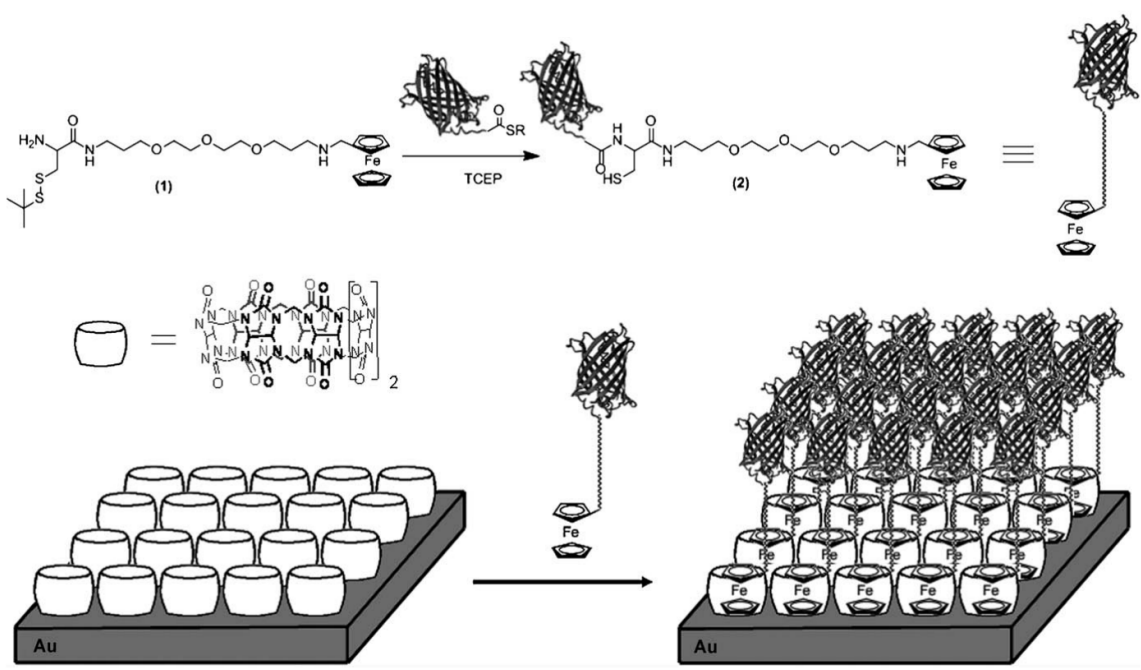

Figure 22: Immobilization of ferrocene ligated yellow fluorescent protein on gold surfaces through interaction of ferrocene guests with the CB[7] monolayer. Reproduced from Young et al. ChemBioChem 2010 11, 180-183. ${ }^{210}$ with permission from John Wiley and Sons, Inc.

Localization of ligands $\mathrm{CB}[n]$ adsorbed to gold surfaces have also been used as a platform for immobilizing biomolecules. ${ }^{210}$ In this case, methyl ferrocene was functionalized with a glycol spacer and a cysteine end-group for ligation with a yellow fluorescent protein thioester (YFP) (Figure 22). The protein could then be immobilized on SAMs of $\mathrm{CB}[7]$ on gold surfaces through the strong interactions with the ferrocene moiety. The proteins from the surfaces could be displaced by using a competitive guest, such as (ferrocenylmethyl)trimethyl-ammonium iodide $\left(K=10^{11} \mathrm{M}^{-1}\right)$. This strategy gave access to a strong, reversible and uniform protein immobilization on surfaces. Similarly, SNAP-fusion cyan fluorescent protein functionalized with ferrocene was immobilized on SAMs of CB[7], generating fluorescent patterns on the surfaces. ${ }^{211}$ This method was then developed further to immobilize cells on CB[7]-coated surfaces using cyclic RGD peptide-ferrocene conjugates. ${ }^{212}$ The RGD peptide allows enhanced cell adhesion and wound healing. The studies showed that the cells remained viable on the surfaces when linked via RGD. In addition, a wound assay also showed successful cell recovery within 
8 hours.

$\mathrm{CB}[7]$ has also been used to physisorb unsubstituted methylviologens onto the surface of $\mathrm{TiO}_{2}$ semiconductor nanoparticle films. The redox-active properties of the viologen guests were further used to prepare electrochromic windows that exhibited reversible color switching under applied potential. ${ }^{213}$

\subsection{Guest-mediated interactions}

Direct supramolecular interactions of surfaces with $\mathrm{CB}[n]$ has undoubtedly opened up avenues for various promising applications, but controlling these spontaneous interactions remains challenging. One strategy, which is widely being reported to gain precise control over the behavior of $\mathrm{CB}[n]$ near surfaces, is to covalently functionalize the surfaces with molecular structures containing guest moieties. The guest mediated interaction of $\mathrm{CB}[n]$ with the surfaces also provide additional handles for stimuli-responsive and reversible architectures, and is particularly attractive for designing colloidal systems.

The earliest example of such a system was illustrated through the construction of a pseudorotaxane self-assembled monolayer on a planar gold surface using CB[6]. The molecular thread was anchored on a gold surface through a 1,2-dithiolane functionality, whereas its diaminobutane unit allowed binding to $\mathrm{CB}[6]$ (Figure 23). The threading of $\mathrm{CB}[6]$ could then be controlled by changing the $\mathrm{pH}$ of the system. Furthermore, the pseudorotaxane acted as a gate to control the access of an electroactive species, $\left[\mathrm{Fe}(\mathrm{CN})_{6}\right]^{3-}$, to the gold surface. ${ }^{214}$ Similarly, gold nanorods decorated with a hydrophilic CB[7]-based pseudorotaxane have also been prepared. ${ }^{215}$

In another example, this method was used to disrupt aggregation of dye molecules on surfaces where high density Rhodamine B functionalized glass slides showed a higher degree of heterogeneity and fluorescence quenching. The addition of $C B$ [7] reduced the heterogeneity and increased the emission significantly to match that of monomeric Rhodamine B species. ${ }^{216}$ More recently, glassy carbon electrodes were modified with CB[7] 

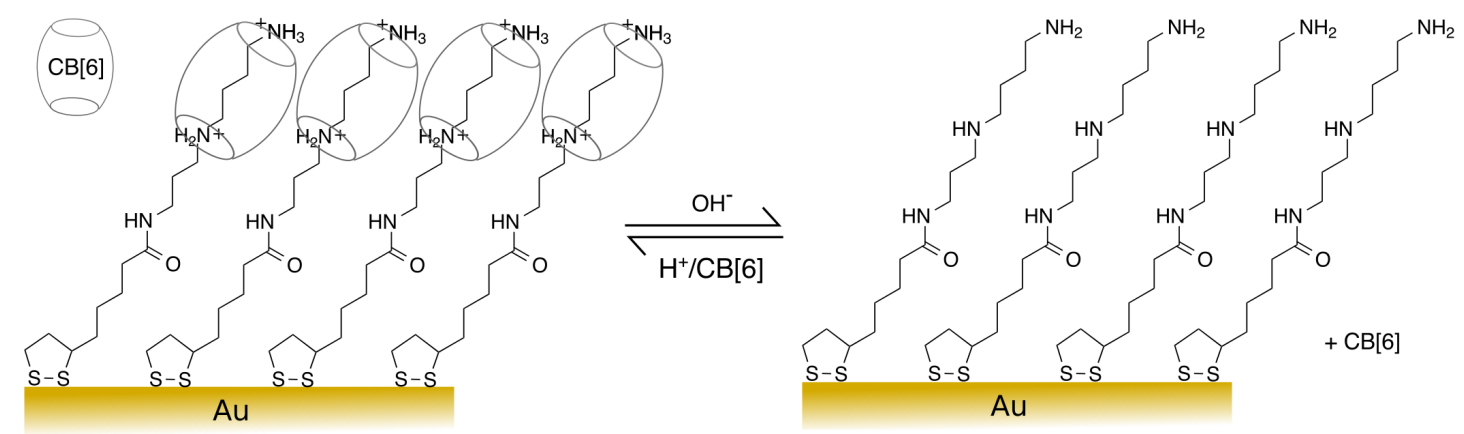

Figure 23: Self-assembled monolayer of pseudorotaxane on gold surfaces and dethreading and rethreading of molecular bead CB[6]. Adapted from Kim et al. Angew. Chem. Int. Ed., 2003, 42, 2395-2398 214 with permission from John Wiley and Sons, Inc.

in order to construct a sensor for glucose sensing using glucose oxidase as a catalyst. ${ }^{217}$ In this case, however, a graphene oxide layer on the electrode was used to enhance the stability of the $\mathrm{CB}[7]$ on the surface. Ferrocenemonocarboxylic acid, the electron transfer mediator can then be captured through molecular recognition by $\mathrm{CB}[7]$. The applicability of this sensor was demonstrated in human serum samples.

Ligand immobilization Based on the finding that $\mathrm{CB}[8]$ is able to induce aggregation in proteins in aqueous solutions, a layer-by-layer construction of homo- and heteroprotein stacks on surfaces has been reported (Figure 24). ${ }^{218}$ In this study, a quartz glass substrate was first modified with amino groups using 3-aminopropyltriethoxysilane. The formation of multiple layers of hemoglobin was then achieved by alternately immersing the substrate in solutions of $\mathrm{CB}[8]$ and protein. The $\mathrm{CB}[8]$ acted as a 'glue' within the proteins stacks. The lack of need to pre-treat or modify the proteins was particularly highlighted in this work by verification of this strategy with several unmodified proteins such as catalase, glutathione-S-transferase agents and insulin. Recently, Rotello and co-workers were able to regulate the interactions between green fluorescent protein and monolayer coated gold nanoparticles using $\mathrm{CB}[7] .{ }^{219}$ The threading of $\mathrm{CB}[7]$ on the surfaces of the nanoparticles led to an increased affinity for the proteins. The authors consider this to be an outcome of the increased cationic charge on the nanoparticle surface based on the slightly positive charge on the outer surface of the equatorial region of 
$\mathrm{CB}[7]$. However, potential repulsion from the negatively charged carbonyl portals was not accounted for in this proposed mechanism.

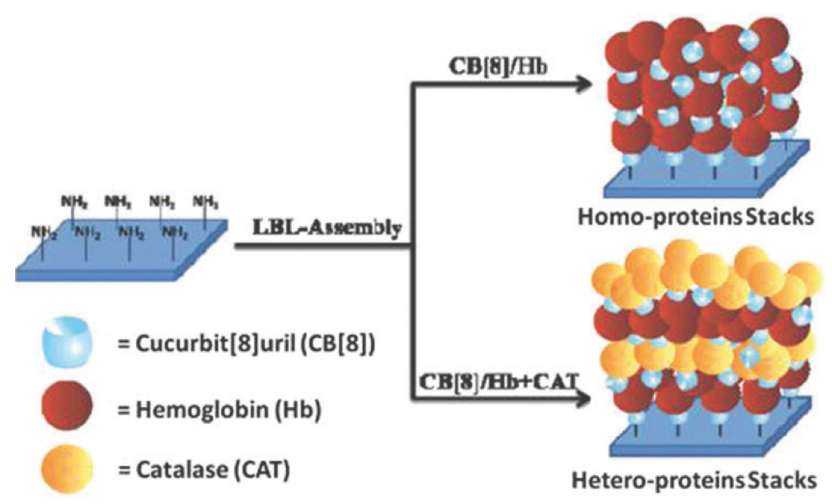

Figure 24: Layer-by-layer construction of protein stacks on surfaces using CB[8] 'glue' molecules. Reproduced from Yang et al. Chem. Commun. 2012 48, 10633-10635 ${ }^{218}$ with permission from the Royal Society of Chemistry.

Scherman et al. have reported the utility of viologen functionalized gold substrates using $\mathrm{CB}[8]$ as a supramolecular 'handcuff' for patterning surfaces with polymeric colloids. Both 1D and 2D patterns were attained. ${ }^{220}$ The same group also described a system where orthogonal switching was achieved controllably and reversibly by using redoxand photo-responsive guest molecules and $\mathrm{CB}[8] .{ }^{221}$ In this system, a SAM of thiol-containing azobenzene derivatives was immobilized on a gold substrate by micro-contact printing. Methyl viologen conjugated with fluorescein dye could be assembled on the surface from solution via $\mathrm{CB}[8]$ 'handcuffs' to afford spontaneously formed 2D-fluorescent patterns. UV-irradiation of the surface converted the azobenzenes to their cis-configuration, which in turn dissociated the second guest from the $\mathrm{CB}[8]$ to turn off the fluorescence. This process could be reversed by irradiation with visible light to covert the azobenzene moieties back to their trans-state. Redox-controlled reversibility of the ternary complex formation provided orthogonal control over the system. In a more recent study, a facile method was reported for the preparation of rotaxanes on gold based on $\mathrm{CB}[8]$ threaded onto a viologen axle, Figure $25 .{ }^{222}$ These surface bound rotaxanes yielded an interlocked structure, which prevented the dissociation of the binary complex $\mathrm{MV}^{2+} \cdot \mathrm{CB}[8]$. The stability of the 
functional surface was thus maintained for binding with electron-rich second guests for $\mathrm{CB}[8]$ such as small molecules like dopamine and pre-functionalized colloidal particles.
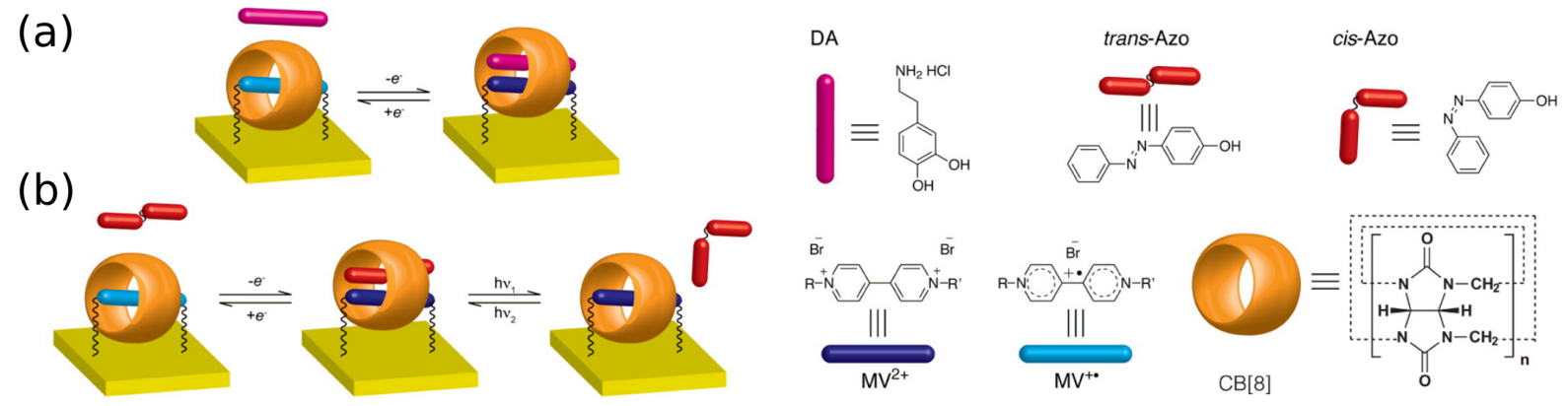

Figure 25: (a) Redox-controlled ternary complexation of $\left(\mathrm{MV}^{2+} \cdot \mathrm{DA}\right) \cdot \mathrm{CB}[8]$ on the Au surface and (b) Redox-controlled and photoresponsive ternary complex formation of $\left(\mathrm{MV}^{2+}\right.$. $\left.\mathrm{Azo}\right) \cdot \mathrm{CB}[8]$ on the Au surface. Adapted from Hu et al. Langmuir 2014 30, 10926-10932. ${ }^{222}$ Copyright 2014 American Chemical Society.

Self-assembled architechtures The ternary complex formation ability of $\mathrm{CB}[8]$ has been used to form and control end-to-end assemblies of gold nanorods. ${ }^{223}$ This was accomplished by first preparing viologen end-functionalized gold nanorods. Then telechelic linker molecules carrying second guest moieties were added to these gold nanorods in the presence of $\mathrm{CB}[8]$ to form aligned chains. The rigidity of the resulting chains could be controlled by changing the length of the linker molecules. Another example of reproducible SERS with colloids was demonstrated using gold nanoparticles pre-coated with cystamine using $\mathrm{CB}[6]$ as linkers to generate gold nanoparticle dimers. ${ }^{224}$

A colorimetric assay for protein detection was reported based on $\mathrm{CB}[8]$-induced formation of bulky aggregates of functionalized gold nanoparticles. ${ }^{225}$ This was achieved by modifying the gold nanoparticles with peptides containing N-terminal aromatic residues, which can be accommodated inside the $\mathrm{CB}[8]$ cavity. $\mathrm{CB}[8]$ is only able to bind to the peptide in the absence of the target protein, inducing a visible color change in the gold nanoparticle solution. The feasibility of this system has been shown in spiked serum samples with a detection limit of $0.2 \mathrm{nM}$.

$\mathrm{CB}[8]$ has also been used to link methyl viologen functionalized polymeric nanoparticles onto silica cores decorated with 4-hydroxyazobenzene moeities, to yield hybrid 'rasp- 
berry' colloidal structures in water (Figure 26). ${ }^{226}$ The presence of azobenzene groups allow reversible assembly and disassembly of the constructs with photo irradiation.

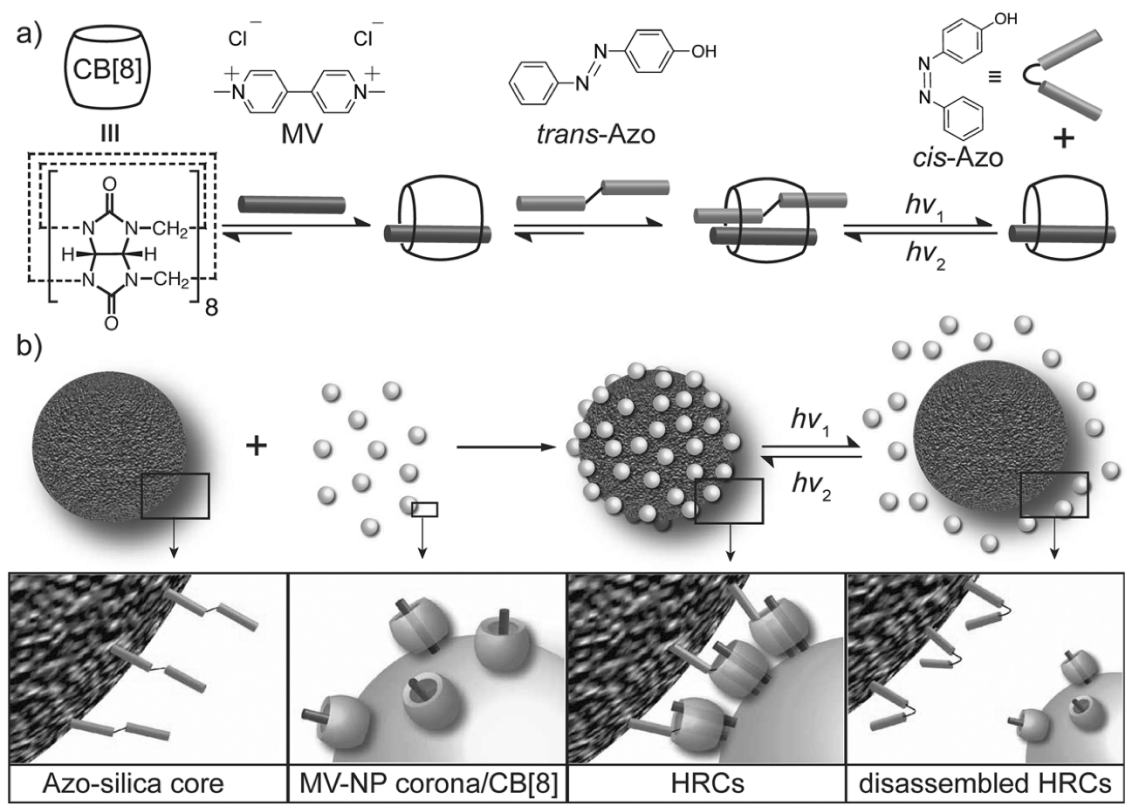

Figure 26: Formation and reversible assembly of hybrid raspberry colloids through CB[8] ternary complexes. Reproduced from Lan et al. Angew. Chem. Int. Ed. 2014 53, 2166-2169 with permission from John Wiley and Sons, Inc.

Nanovalves Pioneered by Stoddart and co-workers, CB[n]-based pseudorotaxane systems on mesoporous silica has made substantial contributions in the field of gated nanochemistry. ${ }^{227-230}$ The release of molecular cargo from mesoporous silica nanoparticles (MNPs) are achieved by blocking and unblocking the silica pores by using $\mathrm{CB}[n]$ as a gating moiety. Early reports were based on $\mathrm{pH}$ dependent actuation of the MNPs. ${ }^{231}$ Increasing levels of sophistication were steadily introduced to these systems by addition of features such as tuning of the $\mathrm{pH}$ at which the MNPs responded, ${ }^{232}$ dual-stimuli constructs capable of exhibiting logic ${ }^{233}$ and magnetic activation. ${ }^{234}$ Later, CB[7]-based gating behavior $^{235,236}$ and alternative approaches to activate the nanovalves were also published. These include competitive binding, ${ }^{235}$ enzyme-triggered systems, ${ }^{236}$ reductive chemistry (cleavage of disulfide bonds) ${ }^{237}$ and light-response. ${ }^{238}$ 


\subsection{Covalent immobilization}

Revefersibility in the binding of $\mathrm{CB}[n]$ to surfaces may not always be desirable in order to achieve better stability and recovery of functionality of the surfaces. Evidently, among the various strategies that have been explored to link $\mathrm{CB}[n]$ and surfaces, the ability to functionalize $\mathrm{CB}[n]$ has been pivotal in addressing these requirements.

Covalently anchored CB[7] on gold surfaces was first reported for the construction of a glucose sensor. ${ }^{239}$ The system was constructed by attachment of allyloxy-CB[7] on vinyl-terminated alkanethiolate SAMs using olefin cross-metathesis. Ferrocenylated glucose oxidaze (GOx) was subsequently immobilized on the surface by means of strong Fc.CB[7] host-guest interactions. Glucose concentrations could then be monitored electrochemically using this construct. The modification of quantum dots with functionalized $\mathrm{CB}[6]$ using thiol linker groups has been reported to significantly improve the chemical and physical stabilities of quantum dots. ${ }^{240}$

In another example, the utility of functionalized $\mathrm{CB}[n]$ on surfaces was elegantly demonstrated by Kim and co-workers in the development of synthetic adhesives, which are functional even under water. ${ }^{104}$ Their velcro-type system is based on a 'hook-and-loop' approach where a silicon surface functionalized with $\mathrm{CB}[7]$ was used to loop around the guest 'hooks' immobilized on a second silicon surface. In order to immobilize the CB[7], alkene functionalized monoallyloxy-CB[7] was attached to thiol-decorated Si surfaces in a thiol-ene reaction. The guests 1,1'-bis(trimethylammoniomethyl)ferrocene $(\mathrm{BFc})$ and aminomethylferrocene $(\mathrm{Fc})$ were chosen on account of their particularly high binding affinities for $\mathrm{CB}[7]$ in water at $10^{12}$ and $10^{15} \mathrm{M}^{-1}$, respectively. Tunability of adhesiveness was achieved through varying the densities of 'hooks' and 'loops' on the surfaces to control the number of multivalent interactions between the two silicon surfaces. A reasonable level of reversibility was also shown with this adhesive through both chemical and mechanical means. It is noteworthy that water molecules often act as a contaminating agent in conventional synthetic adhesives for use under water. On the contrary, this par- 
ticular $\mathrm{CB}[n]$-based approach relies on the expulsion of water molecules in the formation of the $\mathrm{CB}[7]$-guest complexes to fabricate the desired synthetic adhesives.

$\mathrm{CB}[n]$-anchored silica The use of $\mathrm{CB}[n]$ anchored silica has been widely reported in separation science. The production of $\mathrm{CB}[n]$-based stationary phase materials and their use in chromatography was disclosed in patents by Kim and co-workers. ${ }^{241}$ The separation of several organic compounds, such as alkaloids, alkanes, aromatic hydrocarbons, alcohols, esters, ketones and amines using silica gel grafted with perhydroxy-CB[6] has been described in the literature. ${ }^{242,243}$ However, this method suffers from the presence of excess potassium sulfate, which hinders immobilization. In order to address this drawback, another method to fabricate functionalized silica using perallyloxy-CB[6] has also been developed. ${ }^{244}$ Additionally, silica- $\mathrm{CB}[n]$ hybrids prepared using a sol-gel approach also yields stationary phases for efficient chromatographic separations. ${ }^{245-247}$

As an alternative to using covalently modified $\mathrm{CB}[n]$, another method to functionalize $\mathrm{CB}[7]$ on surfaces has been reported using a photochemical reaction with azido groups (Figure 27). ${ }^{248}$ In this strategy, silica substrates were first modified with 3-amino-propyltri-ethoxy-silane to introduce amino groups on the surface. The primary amine was then reacted with 4-azidobenzoic acid, in the presence of 1-(3-dimethylaminopropyl)-3ethylcarbodiimide hydrochloride at basic $\mathrm{pH}$. The azido groups were then exposed to $\mathrm{CB}[7]$ and reacted under UV irradiation for $10 \mathrm{~min}$ to form covalent linkages. While the mechanism of the functionalization could not be elucidated, the authors propose the formation of the highly reactive singlet nitrenes from the azido groups upon UV irradiation. This is followed by their subsequent insertion into the equatorial $\mathrm{C}-\mathrm{H}$ bonds of $\mathrm{CB}[7]$.

Lastly, functionalization of electrodes for better analytical performance has been demonstrated using $\mathrm{CB}[n]$. One such study particularly exploits the molecular recognition properties of $\mathrm{CB}[8]$ to enhance the selectivity and sensitivity of the electrochemical determination of tryptophan. ${ }^{249}$ In brief, the glassy carbon electrode was modified by exposing it to a suspension of Nafion and $\mathrm{CB}[8]$. The nature of interaction with $\mathrm{CB}[8]$ in the construction 


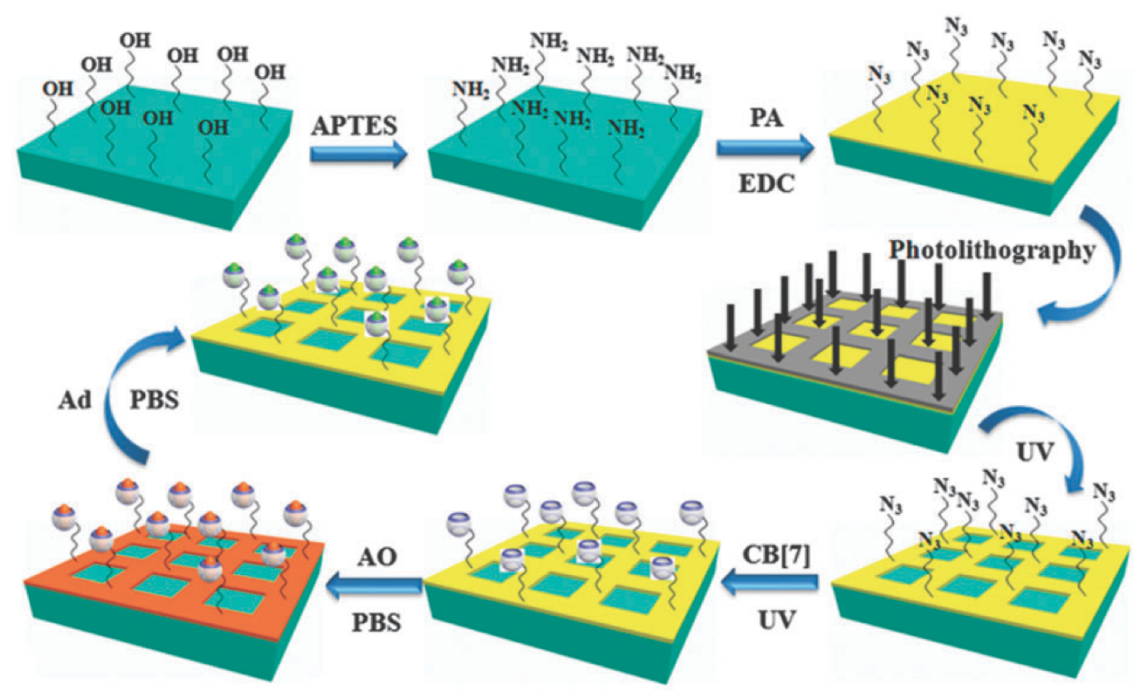

Figure 27: Immobilization of $\mathrm{CB}$ [7] on a silica substrate through photochemical reaction with azides. (APTES) 3-aminopropyltriethoxy-silane, (PA) 4-azidobenzoic acid, (EDC) 1-(3-dimethylaminopropyl)-3ethylcarbodiimide hydrochloride, (AO) Acridine Orange, (Ad) 1-adamantanecarboxylic acid. Reproduced from Zhu et al. Chem. Commun. 2013 49, 8093-8095. with permission from the Royal Society of Chemistry.

of this system, however, has not been studied and remains unclear. Studies with common interferents such as acetylcholine (ACh), ascorbic acid (Asc) and different amino acids namely, D-phenylalanine (Phe), L-tyrosine (L-Tyr) or L-cysteine (L-Cys), arginine (Arg) and lysine (Lys) showed that Phe, Arg and Lys showed a decrease in the electrochemical signal when present at a minimum of 5-fold concentrations to that of Trp, while L-Tyr could be determined simultaneously with the concentration of Trp. The system was also shown to work in human serum, but there remains room for improvement in the reproducibility for real-life applications.

\subsection{Characterization of $\mathrm{CB}[n]$ host-guest binding on surfaces}

In the systems involving $\mathrm{CB}[n]$ and surfaces, the binding properties of the $\mathrm{CB}[n]$ is usually studied in solution and it is assumed that $\mathrm{CB}[n]$ retains its molecular recognition behavior on surfaces. However, with the availability of sophisticated tools such as force spectroscopy, these interactions can now be quantified down to the single-molecule level even on surfaces. For example, atomic force microscopy (AFM) has been used to measure 
the rupture force between individual host-guest complexes of $\mathrm{CB}[6]$ covalently tethered on gold substrates and a spermine functionalized AFM-tip. ${ }^{250} \mathrm{~A}$ rupture force value of $120 \mathrm{pN}$ was obtained for this system. In another study, the interactions between selfassembled monolayers of $\mathrm{CB}[7]$ and neutral adamantyl guests has also been resolved at the single molecule level using dynamic force spectroscopy. ${ }^{251}$ It is noted from such studies that the close proximity of a surface does not appear to significantly alter $\mathrm{CB}[n]$ molecular recognition properties. Furthermore, the spontaneous adsorption of $\mathrm{CB}[6]$ and $\mathrm{CB}[7]$ on gold have been compared by AFM. ${ }^{252}$

\subsection{Outlook}

Direct interactions between $\mathrm{CB}[n]$ and gold continues to show progress in self-assembly of colloidal nanoparticles and sensing applications, particularly in SERS-based applications. The tunability of these systems are one of their major advantages, where the probe can be adapted to the sample. Nonetheless, there remains a need to further explore and elucidate the interactions of $\mathrm{CB}[n]$ with gold on planar surfaces. With advances in techniques like AFM and STM, while this may be possible, a major challenge to be addressed will be to understand and characterize the fundamentals of the formation of colloidal selfassembled architectures because of their increasing complexity. It is noted that nanoparticles that have been synthesized using $\mathrm{CB}[n]$ as the capping ligand have been limited in their size to approximately below $\sim 10 \mathrm{~nm}$ in diameter. While nanoparticles with small diameters might be exceptionally suited for catalysis, larger nanoparticles are much more applicable in plasmonics.

As it is evident from the wide variety of systems being reported, formation of covalent links between $\mathrm{CB}[n]$ and the surfaces is moving fast towards interesting applications. However, these strategies still often require time-consuming pre-functionalized $\mathrm{CB}[n]$. Therefore, the use of host-guest interactions to immobilize CB[6] and CB[8] on surfaces continues to be a promising strategy, where irreversibility is desirable. 


\section{Supramolecular Polymers}

$\mathrm{CB}[n]$ supramolecular polymers can be divided into two areas: (a) small-molecule based supramolecular polymers and (b) CB-mediated bridging of polymer chains and other macromolecular structures. There are a number of excellent reviews in the area of $\mathrm{CB}[n]$ supramolecular polymers and the more general host-guest driven polymerization. ${ }^{253-258}$ In this section, we focus on summarizing the current status of research and development towards $\mathrm{CB}[n]$-supramolecular polymers and highlight some relevant examples in the field, largely dominated by $\mathrm{CB}[8]$ complexes, which has attracted increasing interest in recent years.

\subsection{Small molecule based supramolecular polymers}

The construction of $\mathrm{CB}[n]$ non-covalent polymers based on low molecular weight monomers is analogous to a polycondensation reaction ${ }^{259-261}$ and involves the binding of small molecule guest molecules through $\mathrm{CB}[n]$ host-guest interactions where $\mathrm{CB}[n]$ acts mainly as a "molecular handcuff" holding together the repeating units of the supramolecular polymer. There exist several design parameters that must be considered in order to produce systems that take full advantage of the properties of $\mathrm{CB}[n]$ and generate dynamic polymeric constructs with controlled molecular weight and structure.

The degree of association of supramolecular moieties is strongly affected by both their concentration in solution and their equilibrium constant, and is approximately proportional to $\left(\mathrm{K}_{e q} \cdot \mathrm{C}\right)^{1 / 2} .{ }^{262}$ Therefore, both the concentration and the binding strength between the repeating units have to be high in order to achieve a high degree of polymerization. The solubility of $\mathrm{CB}[n]$ and their complexes varies largely across this family of host molecules - $\mathrm{CB}[5]$ and $\mathrm{CB}[7]$ are much more water soluble than $\mathrm{CB}[6]$ and $\mathrm{CB}[8]^{15}$ and depends on a number of parameters including solvent, temperature, ionic strength and guest. The majority of investigations on $\mathrm{CB}[n]$ supramolecular polymers involve 
$\mathrm{CB}[8]$ complexes, which typically exhibit a limited solubility in water. The advantages afforded by the high binding constants of $\mathrm{CB}[\mathrm{n}]$ complexes, however, supersede the potential drawbacks from low water solubility.

Another key consideration associated to such dynamic systems is the formation of macrocycles and the presence of ring-chain or ring-ring equilibria, which will impact the size of the polymeric aggregates that can be obtained. ${ }^{263-265}$ As with all equilibrium polymerization, a percentage of the chains will be cyclized and the distribution of rings vs. linear chains will depend on a number of factors including monomer shape, length and flexibility, strength of the binding motif, concentration and temperature.

The third consideration is related to the binding mode between repeating units. There is a myriad of "complementary" or "self-complementary" binding motifs that have been utilized for the preparation of supramolecular polymers. ${ }^{262}$ Complementary motifs can be either two-component or three-component, whereby a third moiety is required for association. Some metal-ligand systems including 2,6-di(2-pyridyl)pyridine and 1,10phenanthroline constitute a three-component binding whereby two moieties of the same type are complexed by a single metal ion. Indeed, metal-ligand interactions have been widely exploited and have afforded a facile synthesis of linear and branched supramolecular polymers. ${ }^{266} \mathrm{CB}[8]$ is a relatively large member of the $\mathrm{CB}[n]$ family and can bind two organic guests simultaneously. Kim and coworkers have shown that a stable ternary charge-transfer complex between MV, as a first guest, and 2,6-dihydroxynaphthalene, as a second guest, readily forms inside the cavity of $\mathrm{CB}[8] .{ }^{128}$ Furthermore, the ternary complex is known to form in a stepwise manner with two subsequent equilibria. This three component binding motif is rather unique and reflects molecular recognition of complementary moieties in the presence of a macrocyclic host molecule. In addition, $\mathrm{CB}[8]$ can also accommodate two molecules of the same kind including coumarin, ${ }^{152}$ anthracenyl, ${ }^{136}$ and naphthyl ${ }^{156}$ derivatives. $\mathrm{CB}[10]$ and nor-seco-CB[10], similarly to $\mathrm{CB}[8]$, can bind two organic guests simultaneously inside their cavities and their application in the 
area of aqueous supramolecular polymerization, although with limited success, have also been explored. ${ }^{267,268}$ Nevertheless, it is without a doubt that progress in creating novel $\mathrm{CB}[n]$ mediated polymers has been so far strongly tied to the discovery of new $\mathrm{CB}[8]$ complexes and novel guest design. A facile route to small molecule $\mathrm{CB}[8]$ guest polymerization involves the covalent attachment of a first guest to a second guest moiety. Alternatively, a monomer equipped with two moieties that can be dimerized inside CB[8] can also yield linear polymeric structures. A third option is based on an equimolar mixture of two complementary divalent monomers - first and second guest dimers. We next summarize recent research efforts in the area of $\mathrm{CB}[8]$ supramolecular polymers.

Kim and coworkers were the first to investigate the host-guest complex formation between $\mathrm{CB}[8]$ and a guest molecule having both a first and a second guest moiety connected by an organic linker. Several types of supramolecular assemblies were obtained by this process depending on the length and flexibility of the linker between the binding moieties. It was reported that the long and flexible trimethylene linker of D1 favors 1:1 complexation with $\mathrm{CB}[8]$ (Figure 28). ${ }^{269}$ A similar result was obtained for the apparently more rigid but-2-enyloxy linker of D2 (Figure 28). The introduction of an even more rigid p-xylylene linker D3 in Figure 28) effectively prevented 1:1 complexation inside CB[8] but failed in promoting chain extension as only a 2:2 complex was produced. ${ }^{270}$ When CB[8] and monomer D4 were mixed in equimolar amounts ( $2 \mathrm{mM}$, aqueous solution) a mixture of a 2:2 complex and oligomeric species (3:2 ratio) was produced. ${ }^{271}$ The hydrodynamic volume of the oligomeric, likely cyclic, species was estimated by diffusion-ordered NMR spectroscopy and a degree of polymerization of ca. 4 was proposed. Linear supramolecular polymers were successfully grown from a gold surface functionalized with dipyridyliumylethylene units. A surface-anchored poly(pseudorotaxane) was grown by soaking the functional gold surface in an aqueous solution of $\mathrm{CB}[8]$ and monomer D4 $(1 \mathrm{mM}$, water). After a 1 day incubation period, the poly(pseudorotaxane) reached a degree of polymerization of 4 . The surface-anchored poly(pseudorotaxane) was characterized by 
surface plasmon resonance and atomic force microscopy images. Monomer D5 contains a naphthalene and a dipyridyliumylethylene unit connected by a methylene bridge. Although the short linker prevents intramolecular complexation, the structural design again promotes cyclization rather than linear polymer formation. An equimolar mixture of $\mathrm{CB}[8]$ and $\mathrm{D} 5$ resulted in a stable cyclic pentamer comprising five $\mathrm{CB}[8]$ molecules and five guest molecules. ${ }^{272}$

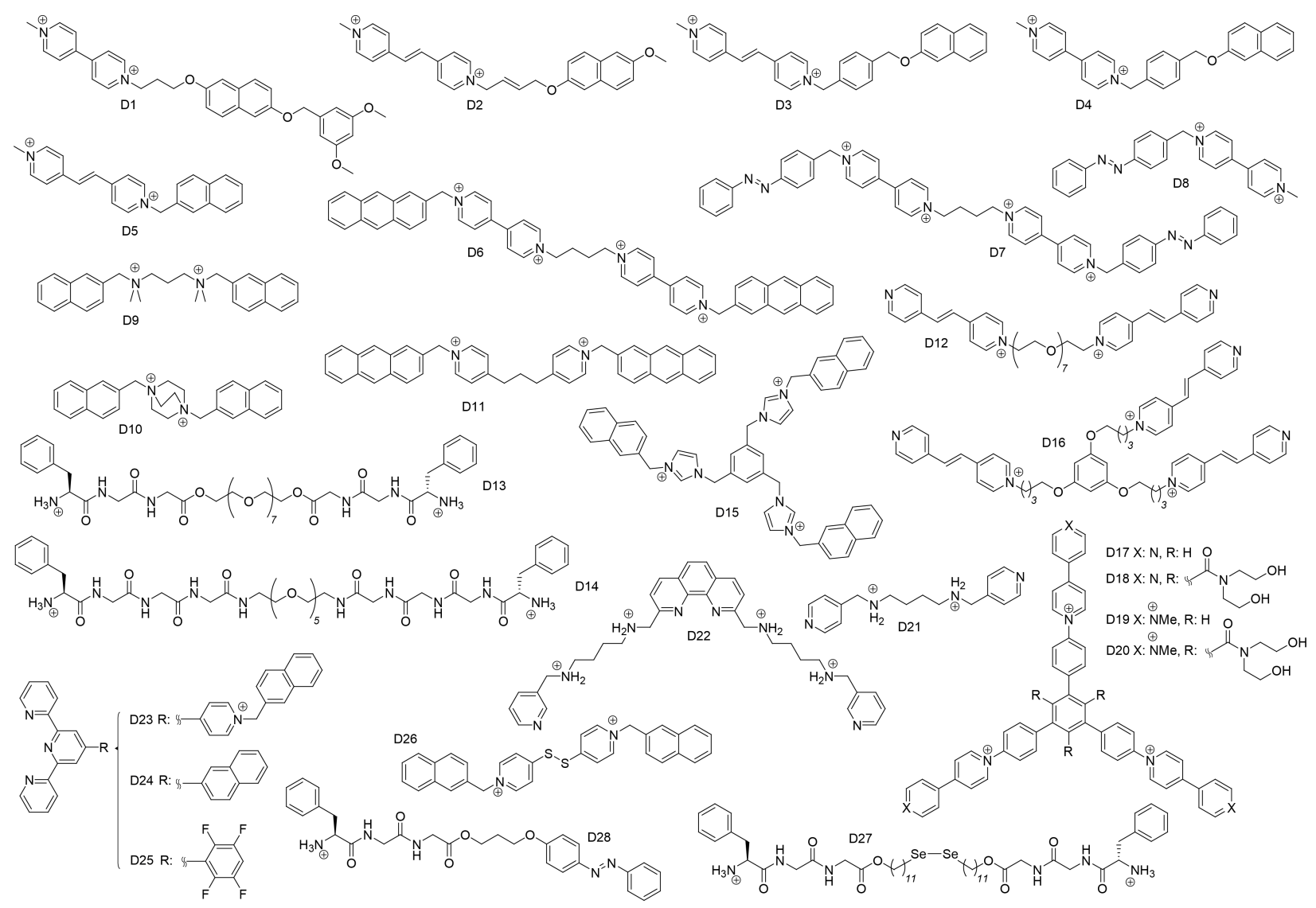

Figure 28: Structures of monomers.

In order to circumvent cyclization, Zhang and coworkers designed a monomer containing up to four $\mathrm{CB}[8]$ binding sites in a single monomeric structure. The structure of such a monomer contains two central viologen moieties and two terminal anthracene groups, providing what was a called a "double handcuff" to hold repeating units together (D6 in Figure 28). ${ }^{273}$ The short methylene linker between the anthracene and the viologen moieties avoids intramolecular complexation while the pair of central viologens 
effectively prevents the formation of 2:2 complexes - the positively charged viologens will sit too close to each other in a cyclic 2:2 complex. When $\mathrm{CB}[8]$ was mixed with D6 in a 1:1 ratio the appearance of a charge-transfer band evidenced heteroternary complex formation. The 1:1 stoichiometry of the complex was corroborated by Job's plot analysis. AFM-based single-molecule force spectroscopy analysis showed the formation of extended polymer chain formation. The force curves of the supramolecular polymer were similar to traditional macromolecules and a Kuhn and chain length of ca. 2.2 and $60.0 \mathrm{~nm}$, respectively were determined [comparatively, the Kuhn length for DNA in water ${ }^{274}$ and poly(tert-butyl methacrylate) ${ }^{275}$ in toluene are approximately 100 and $2 \mathrm{~nm}$, respectively]. Dynamic light scattering experiments provided further evidence of polymer formation and the Rh of the chains was determined to be on the order of $30 \mathrm{~nm}$.

Inspired by the previous monomer design proposed, Scherman and coworkers investigated an analogous guest structure equipped with azobenzene instead of anthracene moieties (D7 in Figure 28). The formation of supramolecular polymers was charaterized both in solution and in the solid state through a combination of dynamic and static light scattering and synchrotron SAXS and SANS, see Figure 29. ${ }^{276}$ Interestingly, the shorter monomer D8 can also form relatively long linear structures in solution, which coexist with the more stable 2:2 complex. These polymers can be readily depolymerized by UV light irradiation as the E-azobenzene moieties isomerize into the Z-isomer that fully occupies the $\mathrm{CB}[8]$ cavity with a high binding constant. The formation of a 1:1 complex between $\mathrm{CB}[8]$ and Z-azobenzene was investigated in solution by ITC and NMR. Additionally, single crystals of the polymeric structure were obtained and analysed by X-Ray crystallography. This light-controlled formation of $\mathrm{CB}[8]$ complexes is substantially different to other light-actuated host-guest systems and may find applications in biological and materials sciences.

Zhang and coworkers have investigated the formation supramolecular polymers based on a wide variety of homotopic divalent monomers containing self-complementary $\mathrm{CB}[8]$ 


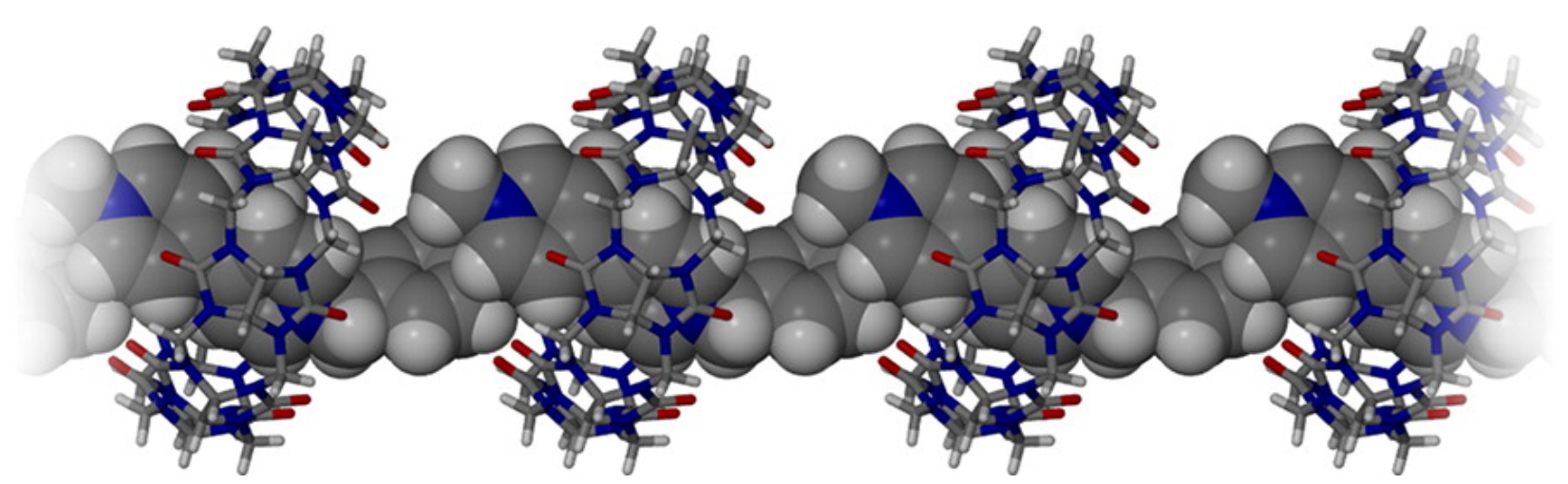

Figure 29: Side view of the X-ray crystal structure of $\mathrm{CB}[8]_{n} \cdot \mathbf{D} 8_{n} . \mathrm{C}$, gray; $\mathrm{N}$, blue; $\mathrm{O}$, red; $\mathrm{H}$, white. Reprinted with permission from del Barrio, J. et al. J. Am. Chem. Soc. 135, 11760-11763 (2013). ${ }^{276}$ Copyright 2013 American Chemical Society.

binding moieties including naphthalene, anthracene, phenylalanine and quaternized azastilbene groups (see D9-D13 in Figure 28). ${ }^{277-280}$ These moieties readily dimerized inside the cavity of $\mathrm{CB}[8]$ thus promoting polymerization. Monomers D9 and D10 contain two naphthalene moieties in close proximity to positively charged quaternary amines. ${ }^{278}$ The flat aromatic groups can form host-guest enhanced $\pi-\pi$ complexes in the presence of $\mathrm{CB}[8]$ and the positive charges ensure the water solubility of the final polymer and further stabilization through ion-dipole interactions with the ureidyl carbonyl portals. While D9 only formed cyclic oligomeric species in the presence of CB[8], D10 resulted in long, linear polymeric structures. This was attributed to the rigid, shape persistent 1,4-diazabicyclo[2.2.2]octane-1,4-diium linker, which can effectively inhibit cyclization. The degrees of polymerization estimated by DOSY NMR spectroscopy were on the order of 64 for the polymer based on D10 and 7 for the oligomeric species derived from D9 (1 $\mathrm{mM}$, water), thus highlighting the crucial role of the monomer spacer. The same group nevertheless showed that other monomers D12 and D13 (Figure 28) with much longer and flexible linkers than D10 can also polymerize. One interesting example is provided by the comparison of monomers D13 and D14. Zhang and coworkers investigated the supramolecular polymerization of D13 in the presence of $\mathrm{CB}[8]$ based on MALDI-TOF mass spectrometry and AFM-based single-molecule force spectroscopy. Both techniques 
evidenced the formation of polymers with a degree of polymerization of the order of 2060. Dankers and coworkers have recently investigated the binding properties of D14, very similar to D13, through a combination of ITC, DOSY NMR spectroscopy and QTOF and MALDI-TOF mass spectrometry and found no evidence of polymer formation. ${ }^{281}$ Data suggests that ring-chain equilibrium is in fact fully directed to ring formation (1:1 complex). The formation of this complex can by described by an overall binding constant $\mathrm{K}_{\text {ring }}=9.0 \times 10^{6} \mathrm{M}^{-1}$ and an effective concentration of $750 \mu \mathrm{M}$. The well-defined ring structure of the cyclic dimer D14.CB[8], with a high equilibrium constant, provided a very efficient inhibition mechanism for $\mathrm{CB}[8]$ protein dimerization processes as it can readily displace phenylalanine-containing peptide sequences from different proteins. ${ }^{282}$ MALDI-TOF analysis of CB[8] polymers from both D13 and D14 revealed peaks at high molecular weight, which were attributed to supramolecular polymer formation by Zhang and coworkers, whereas Dankers and coworkers ascribed them to $\mathrm{CB}[8]$ aggregates artefacts.

The concept of $\mathrm{CB}[8]$ homoternary complexation has also been expanded towards the preparation of hyperbranched polymer structures, networks and 2D polymer networks from monomers equipped with at least 3 binding sites (D15, D16, D18 in Figure 28). ${ }^{283-287}$ It is noteworthy that, again, the flexibility of the monomer will determine the final structure of the polymers: monomers with flexible linkers (D15 and D16) will result in hyperbranched polymers whereas $2 \mathrm{D}$ networks are produced from the more rigid monomer D18.

Another interesting concept consists of exploiting $\mathrm{CB}[8]$ enhanced radical dimerization as a driving force in supramolecular polymerization. Kim and coworkers reported in 2002 that methyl viologen binds to $\mathrm{CB}[8]$ in 1:1 stoichiometry with a binding constant on the order of $10^{5} \mathrm{M}^{-1} .{ }^{142}$ When methyl viologen is reduced to the radical cation, a 1:2 complex is formed exclusively. The apparent binding constant for the dimerization process is on the order of $10^{7} \mathrm{M}^{-1}$. This homoternary complex is extraordinarily stable on account 
of extensive ion-dipole interactions between the ureidyl carbonyl portals of $\mathrm{CB}[8]$ and $\mathrm{MV}^{\bullet+}$. In addition, the host provides the dimer with a hydrophobic cavity of the right size to host the couple of radical-cations. Thus, the presence of $\mathrm{CB}[8]$ affords a convenient, simple and reversible mechanism to drive self-assembly of a variety of organic moieties. Kaifer and coworkers reported in 2004 that the complexation-enhanced dimerization of viologen radical-cations can be utilized to self-assemble Newkome-type dendrons equipped with a MV moiety at their focal point. ${ }^{288}$ Zhan-Ting Li, Yi Liu and coworkers have recently described the formation of 2D supramolecular organic frameworks from shape-persistent viologen-containing trimers (monomers D19 and D20). ${ }^{286}$ The viologen moieties of the trimers can be easily reduced in the presence of sodium dithionite thus inducing self-association in water. The reduced trimer D19 stacks strongly in a face-to-face fashion forming discrete $2+3$ complexes with $\mathrm{CB}[8]$. However, the presence of the bulky bis(2-hydroxyethyl) carbamoyl groups of D20 avoids face-to-face stacking thus leading, after reduction, to the formation of a single-layer 2D supramolecular organic framework, which is stabilized by the strong complexation of $\mathrm{CB}[8]$. The new supramolecular networks were characterised by UV/vis absorption, EPR, DLS, as well as solution and solidphase small-angle X-ray diffraction experiments. The single-layer feature of the network was confirmed by AFM imaging as the average thickness of the supramolecular framework was consistent with the diameter of $\mathrm{CB}[8]$. Analogous monomeric trimers derived from 4,4-bipyridin-1-ium (monomers D17 and D18) also self-assemble in the presence of $\mathrm{CB}[8]$ in a similar fashion to the previously described reduced trimers, Figure 30. ${ }^{287}$

In 2004, Kim and coworkers described the first stable $\pi$-dimer of a tetrathiafulvalene radical-cation encapsulated inside the cavity of $\mathrm{CB}[8] .{ }^{289}$ This was demonstrated through a combination of NMR, UV/vis and EPR spectroscopy. TTF binds to CB[8] and is simultaneously oxidized to the radical-cation species forming a stable three-component complex as a red precipitate. Although no quantitative binding study was performed, this work demonstrated the effectiveness of $\mathrm{CB}[8]$ in stabilizing unstable species through molecu- 


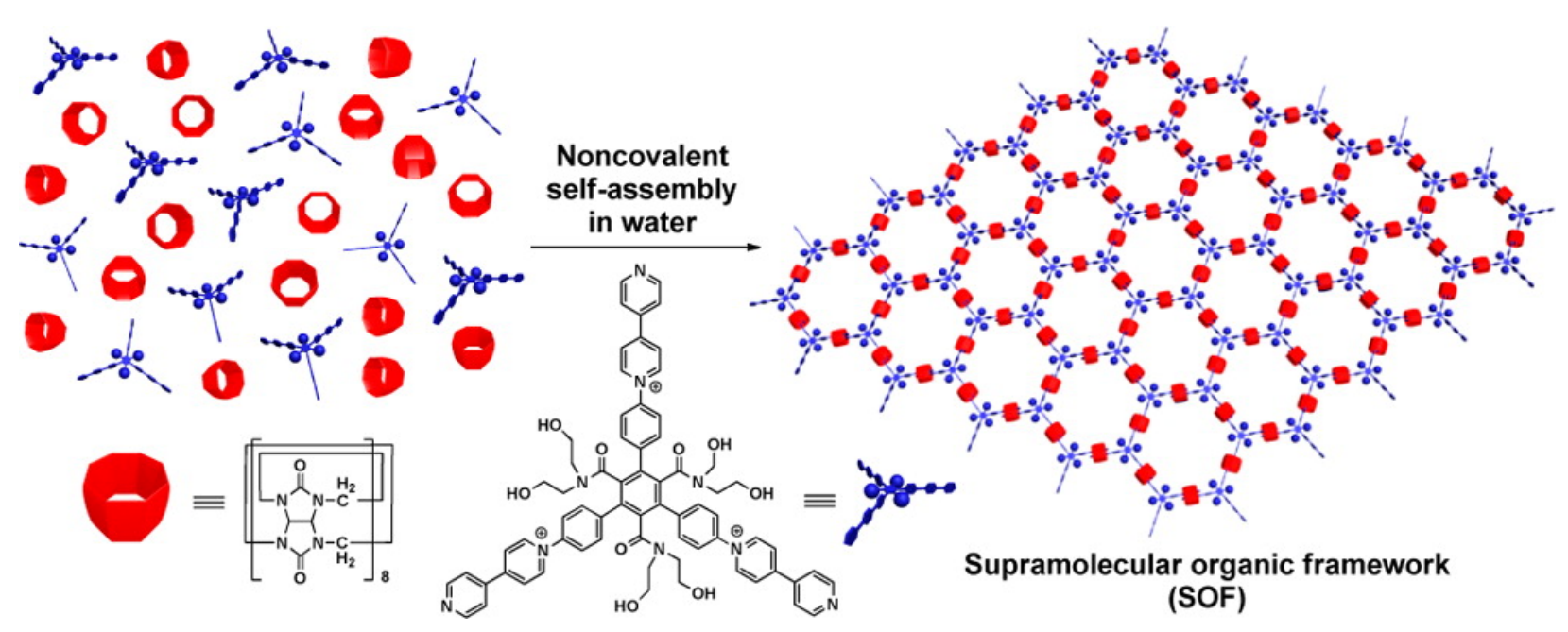

Figure 30: Schematic representation of the self-assembly of monomer D20 and CB[8]. Reproduced from Ref. ${ }^{286}$ with permission from The Royal Society of Chemistry.

lar encapsulation. TTF radical dimerization has been shown to be extremely useful in exerting redox control over a wide variety of polymeric structures and mechanically interlocked constructs. ${ }^{290-294}$ However, supramolecular polymer formation driven by encapsulation of the TTF radical-cation inside the cavity of $\mathrm{CB}[8]$ has not been realized yet.

Supramolecular polymers constructed by orthogonal self-assembly based on hostguest, including $\mathrm{CB}[n] \mathrm{s}$, and other dynamic covalent/non-covalent interactions are attracting increasing attention. ${ }^{295,296} \mathrm{Kim}$ and coworkers pioneered the area $\mathrm{CB}[n]$ orthogonal self-assembly by designing a wide variety of metal-organic $\mathrm{CB}[n]$ polyrotaxanes. ${ }^{297}$ One of these structures consists of a pseudorotaxane-prepared by a simple mixture of D21 and $\mathrm{CB}[6]-$ that yields a linear polymer in the presence of $\mathrm{Cu}^{2+}$ through pyridine-metal ion coordination. ${ }^{298}$ A polycatenated 2D polyrotaxane net is formed, however, if $\mathrm{Ag}^{+}$is used instead of $\mathrm{Cu}^{2+} .{ }^{299}$ The same strategy combined with a more complex ligand D22 produces double-chained and zigzag-shaped 1D polyrotaxanes in the presence of $\mathrm{Cd}^{2+}$ and $\mathrm{Co}^{2+} .300$ The repeating units of these metal-organic structures are held together by metal-ligand, not host-guest, interactions; however the $\mathrm{CB}[n]$ complex plays a crucial role as it imparts rigidity to the organic linker. More recently, Zhang and coworkers investigated the preparation of $\mathrm{CB}[8]$ supramolecular polymers through a combination of host- 
guest and terpyridine- $\mathrm{Fe}^{2+}$ interactions. ${ }^{301}$ Monomer D23 is a heteroditopic molecule consisting of a naphthalene and terpyridine moiety which can be dimerized in the presence of $\mathrm{Fe}^{2+}$ forming a monomer with two terminal naphthalene moieties, $\left[\mathrm{Fe} \cdot \mathbf{D} 23_{2}\right]^{2+}$. This new metal-containing monomer can undergo polymerization when mixed with an equimolar amount of $\mathrm{CB}[8]$ in a similar fashion to monomer D10. It was claimed that the cationic metallic center enhances the water solubility of the complex while serving as a rigid spacer between the $\mathrm{CB}[8]$ binding sites. Masson and coworkers have further explored this concept and demonstrated that coulombic interactions between metal centers and $\mathrm{CB}[n]$ can actually be mediated by an organic ligand and showed that, even in the second coordination sphere, $\mathrm{CB}[n]$ form strong $\mathrm{CH} \cdots \mathrm{O}$ hydrogen bonds with the ligand surrounding the metallic core. ${ }^{302}$ This recognition process is driven by a combination of favorable enthalpies (up to $20.2 \mathrm{kcal} \cdot \mathrm{mol}^{-1}$ ) and very unfavorable entropic contributions (as low as $-10.2 \mathrm{kcal} \cdot \mathrm{mol}^{-1}$ ). The recognition properties of the metal-ligand complexes were extended to $\mathrm{CB}[8]$ and dynamic supramolecular oligomers were prepared by mixing equimolar amounts of ligand $\mathrm{D} 24$ and $\mathrm{CB}[8]$ in the presence of $\mathrm{Fe}^{2+}$ and $\mathrm{Ir}^{3+}$. The approximate degree of polymerization of the oligomers, based on DOSY NMR spectroscopy, were 11 and 54 for the $\mathrm{Fe}^{2+}$ and $\mathrm{Ir}^{3+}$ polymers $(1 \mathrm{mM}$, water). $\mathrm{CB}[8]$ can also encapsulate 2,3,5,6-tetrafluorophenyl units and supramolecular oligomers were also produced when mixing $\left[\mathrm{Fe} \cdot \mathbf{D} 25_{2}\right]^{2+}$ and $\left[\mathrm{Ir} \cdot \mathbf{D} 25_{2}\right]^{3+}$ with $\mathrm{CB}[8]$ in equimolar amounts (Figure 28). Degrees of polymerization of 27 and 15 were estimated for the $\mathrm{Fe}^{2+}$ and $\mathrm{Ir}^{3+}$ polymers respectively. Also, social self-sorting was observed for an equimolar mixture of $\left(\left[\mathrm{Fe} \cdot \mathbf{D} 24_{2}\right]^{2+} \cdot \mathrm{CB}[8]\right)_{n}$ and $\left(\left[\mathrm{Ir} \cdot \mathbf{D} 25_{2}\right]^{3+} \cdot \mathrm{CB}[8]\right)_{n}$ thus generating well-defined oligomers with alternating $\mathrm{Fe}^{2+}$ and $\mathrm{Ir}^{3+}$ cationic centers, Figure 31. The preference for naphthyltetrafluorophenyl pairing was attributed to favorable quadrupole-quadrupole interactions in a similar fashion to those present in benzene-perfluorobenzene pairs. ${ }^{303,304}$ In addition to metal-organic interactions, other types of dynamic covalent chemistries have been combined with $\mathrm{CB}[8]$ host-guest interactions to produce linear supramolecular poly- 
mers. These include thiol-disulfide, diselenides and cyclodextrin host-guest interactions (monomers D26-D28 in Figure 28). ${ }^{305-307}$ D28, as an example, comprises an azobenzene moiety covalently attached to a phenylalanine residue. The monomer can dimerize in the presence of $\mathrm{CB}[8]$ to form a 2:1 $\mathrm{D} 28_{2} \cdot \mathrm{CB}[8]$ complex, which can undergo subsequent polymerization when mixed with an equimolar amount of a $\beta$-cyclodextrin dimer. ${ }^{307}$

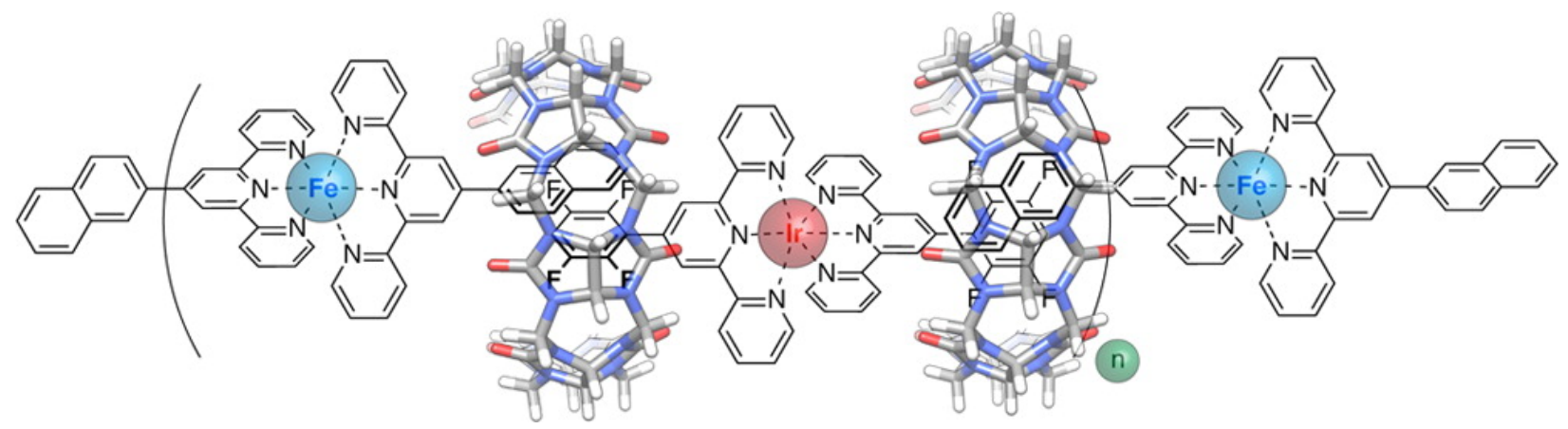

Figure 31: Schematic representation of the alternating polymer resulting from an equimolar mixture of $\left(\left[\mathrm{Fe} \cdot \mathbf{D} 24_{2}\right]^{2+} \cdot \mathrm{CB}[8]_{n}\right.$ and $\left(\left[\mathrm{Ir} \cdot \mathbf{D} 25_{2}\right]^{3+} \cdot \mathrm{CB}[8]\right)_{n}$. Reprinted with permission from Joseph, R., Nkrumah, A., Clark, R. J. \& Masson, E. J. Am. Chem. Soc. 136, 6602-6607 (2014). ${ }^{302}$ Copyright 2014 American Chemical Society.

The concept of $\mathrm{CB}[8]$-mediated phenylalanine dimerization was also exploited by Liu and coworkers in the design and synthesis of protein polymers. ${ }^{308}$ In this case, a homodimer glutathione S-transferase was selected as the building block for genetic fusion with phenylalanine-glycine-glycine oligopeptides at each side of its N-termini. The homodimer protein polymerizes via homoternary complex formation between two phenylalanine residues and $\mathrm{CB}[8]$ as demonstrated by the increase of the molecular weight of the aggregate. The protein-based polymer had a wire-shaped structure with and an average length of $128 \mathrm{~nm}$ (degree of polymerization around 25) and a height of about $4.8 \mathrm{~nm}$. This protein assembly was highly stable and could even undergo separation by gel permeation chromatography and further functionalization with glutathione peroxidase.

Finally, Zhang and coworkers have recently reported a novel polymerization methodology controlled by $\mathrm{CB}[8]$ and $\mathrm{CB}[7]$ self-sorting. ${ }^{309}$ The supramolecular polymerization of a tetracationic monomer composed of $p$-phenylene and naphthalene moieties was pro- 
moted by the selective recognition of the central $p$-phenylene ring by $\mathrm{CB}$ [7] followed by a CB[8] naphthalene double encapsulation. A degree of polymerization of ca. 28 was obtained by asymmetric flow field flow fractionation (that conveniently bypasses the use of GPC stationary phases) coupled to multi-angle light scattering.

\section{2 $\mathrm{CB}[8]$ mediated supramolecular block copolymerization}

Not only is supramolecular polymerization possible by $\mathrm{CB}[8]$ complexation of small molecules, higher molecular weight molecules such as polymers can also be complexed at their termini, or via side chains, to other polymers, small molecules, biomolecules, etc. As the following chapter will demonstrate, this process can lead to large and facile increases in molecular weight, not only affording chain-extended polymers, but by relying on the specific heteroternary 1:1:1 CB[8] stabilized charge transfer complex, self sorted amphiphilic supramolecular block copolymers can be produced allowing formation of micelles and vesicles that can be assembled, disassembled, and reassembled using various stimuli responsive triggers.

\subsubsection{Supramolecular assembly of macromolecules}

Kaifer et al. first demonstrated macromolecular assembly with CB[8] by centrally and non-covalently complexing two distinct dendrimers, one functionalized with MV (electron acceptor) and a second with para-dialkoxybenzene (electron donor). ${ }^{131}$ In this study, dendrimers were selectively assembled on account of the guest covalently attached and the efficiency of this process was shown to be dependant on the size of the corresponding dendritic units. Electrochemical one-electron reduction of viologen resulted in homodimerization of dendrimer units on account of 2:1 complexation of the MV radical cations to $\mathrm{CB}[8]$ and therefore redox switching between discrete molecular weight dynamic dendrimer dimers was possible.

A facile methodology of block-copolymerization by supramolecular complexation is 
that of the dimerization of MV-terminated polymers to Np-terminated polymers by $\mathrm{CB}[8]$ addition, Figure $32 .{ }^{310}$ This lead to the formation of linear dynamic elongated homopolymers and AB-diblock copolymers, as demonstrated by Scherman et al. Dynamic amphiphilic polymers of PEG and cis-1,4-poly(isoprene) or octadecane were prepared and these were also able to form larger vesicular structures in a highly versatile manner.

Ji et al. reported similar aqueous structures of a linear hyper-branched polyphosphate with a terminal MV unit that complexed to $\mathrm{CB}[8]$ and poly(D,L-lactide) with terminal indole units. ${ }^{311}$ This formed an amphiphilic block copolymer structure that would assemble into a biocompatible micellar structure. Disassembly of these micelles was possible by reduction of the MV units with sodium dithionite, breaking the hetero-ternary complex.
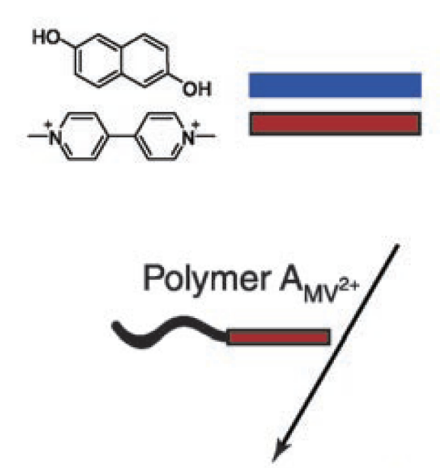

a)

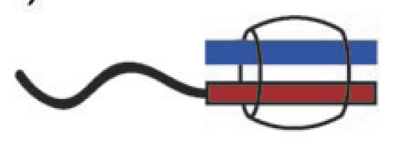

$\mathrm{CB}[8]$-polymer conjugate b)
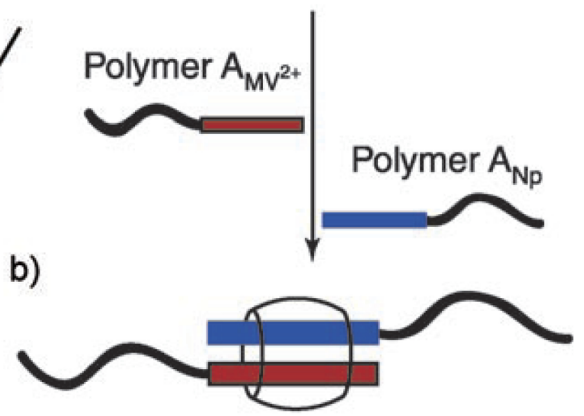

Elongated homopolymer
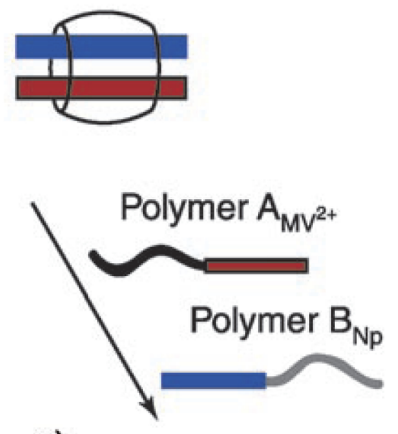

c)

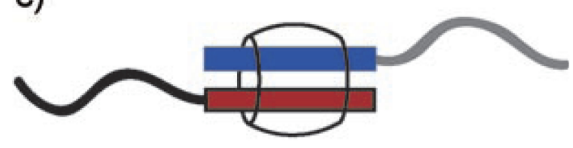

AB diblock copolymer

Figure 32: Host-stabilized charge transfer complexes allow for the formation of a variety of macromolecular materials, from $\mathrm{CB}[8]$-polymer conjugates, elongated or chain extended linear homopolymers and $\mathrm{AB}$ diblock copolymers. Figure reproduced from Rauwald et al. Angew. Chem. Int. Ed., 2008, 47, 3950-3953, with permission from John Wiley and Sons, Inc.

Actual polymeric chain extension by $\mathrm{CB}[8]$ complexation at polymer termini was investigated by Jiang et al., Figure 33a. ${ }^{312}$ Low molecular weight poly( $N, N$-dimethylacrylamide) and poly( $N$-isopropylacrylamide) with naphthyl moieties at both termini were synthe- 
sized via reversible addition-fragmentation chain-transfer (RAFT). These polymers were then combined in solution with a viologen-dimer and $\mathrm{CB}[8]$ to form host-stabilized chargetransfer complexes and higher molecular weight supramolecular polymers from the lower molecular weight building blocks. The existence of these higher molecular weight supramolecular polymers was observed using viscometry techniques. The resultant supramolecular pNIPAm also expressed interesting LCST behavior, that was strongly dependent on concentration (higher concentrations are associated with lower LCSTs), unlike covalent pNIPAm, which exhibits very limited concentration dependence on its critical temperature.

Not only is supramolecular complexation possible at the polymer terminus, indeed Scherman et al. also demonstrated the reversible and stimuli responsive loading of side chains on to hydrophilic polymer chains by utilizing $\mathrm{CB}[8]$ ternary complexation motifs. ${ }^{313}$ A copolymer of oligoethylene glycol acrylate and a naphthol bound monomers was synthesized via RAFT polymerization. MV derivatives were then reversibly complexed to the naphthol residues. In particular, MV-bound hydrophobic residues such as C18 chains could be "pulled" into aqueous solution using this complexation method. Upon addition of sodium dithionite, the MV moieties were reduced from dicationic species to radical cations, breaking the 1:1:1 heteroternary complexes. This process was in turn reversible by bubbling air through the solution, oxidizing the MV and regenerating the charge transfer complexes. This work holds design features amenable to controlled or triggered release delivery devices. Monosaccharides and multivalent ligand-lectins have similarly been non-covalently attached to such copolymers to add bio-recognition modalities. ${ }^{314}$ A supramolecular glycopolymer was produced, which could bind lectins and then be released from the copolymer backbone using redox methods. Such polymer designs could be further used to probe cellular activity and biomarkers. Development of this area has lead to the development of drug delivery applications with both Scherman and Ji reporting the delivery of doxorubicin, a commonly used chemotherapeutic, 

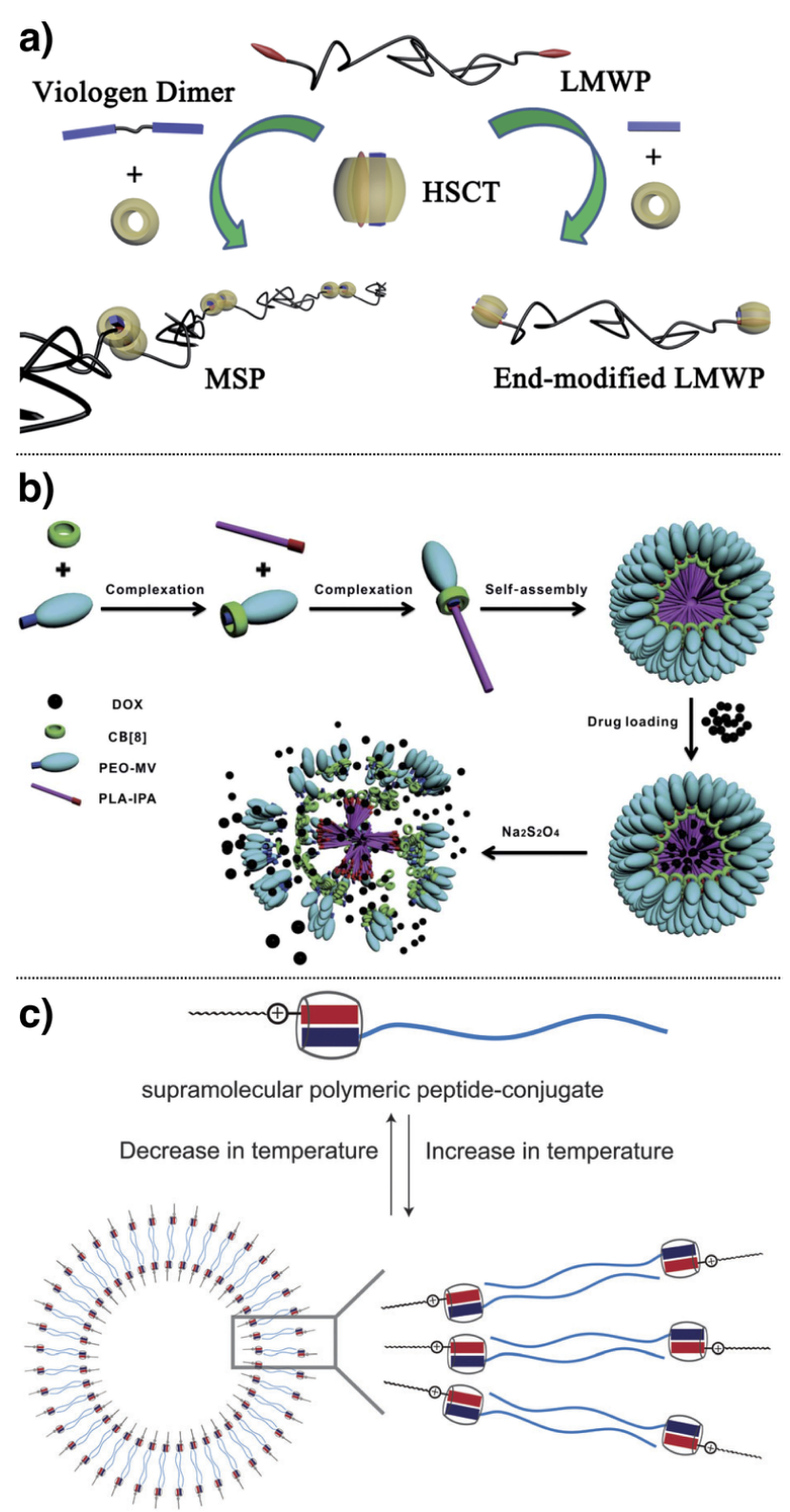

Figure 33: Higher order structures afforded through $\mathrm{CB}$ [8] host-guest chemistry: a) Chain extended polymers by $\mathrm{CB}[8]$ complexation of terminally functionalized polymers to viologen dimers, figure adapted with permissions from Ji et al. Polym. Chem. 2014, 5, 2709-2714. b) Supramolecular assembly of amphiphilic block copolymers leads to micelle formation which have utility in drug delivery, figure adapted with permissions from Ji et al. Polym. Chem. 2014, 5, 1843-1847. c) Aqueous self-sorting of supramolecular polymers allows for vesicle formation, figure adapted with permissions from Loh et al. Chem. Commun. 2014, 50, 3033-3035. All images reproduced with permission from The Royal Society of Chemistry. 
from supramolecular micellar structures. In both cases, $\mathrm{CB}[8]$ hetero-ternary complexation was employed to form supramolecular amphiphilic block copolymers, which would in turn form micelles through hydrophobic interactions, Figure 33b. ${ }^{315,316}$ Scherman et al. constructed triply stimuli-responsive micelles from temperature and chemo-responsive $\mathrm{CB}[8], \mathrm{pH}$ responsive naphthalene terminated poly(dimethylaminoethylmethacrylate) (PDMAEMA$\mathrm{Np}$ ), and temperature responsive MV terminated poly( $N$-isopropylacrylamide) (PNIPAAm$\mathrm{MV})$. Mixing these components in aqueous solution in an equimolar ratio resulted in micelle formation in which doxorubicin was encapsulated. Changes in $\mathrm{pH}$, temperature or addition of the competitive guest, adamantylamine, resulted in disassembly of the micelles and release of doxorubicin, which was shown to have a direct effect on cell viability. Moreover, exposed cell lines exhibited good tolerance of the doxorubicin loaded micelles, only exhibiting detrimental effects upon triggered release. Ji and coworkers reported a similar assembly of micelles utilizing a different hetero-ternary supramolecular motif. In this example, the hydrophobic block consisted of an indole terminated poly(lactic acid) whereby indole is an electron rich moiety utilized in the charge-transfer "handcuff" motif. The hydrophilic block consisted of MV terminated PEO. Furthermore, both poly(ethylene oxide) and poly(lactic acid) have been FDA approved for medical use and the latter also has an excellent biodegradability profile. Again doxorubicin encapsulation was possible within the hydrophobic core of the micelle and release could be triggered by addition of sodium dithionite, reducing the MV and causing disassembly.

Indeed, supramolecular assembly of higher order structures is not limited to micelles and the aqueous construction of double layer vesicles was also reported by Scherman et al. in 2012. ${ }^{317}$ Assembly of a pyrene-GGGKKK peptide with a MV-lipid through hostguest ternary complexation with CB[8] afforded $200 \mathrm{~nm}$ spherical double layered vesicles, observed by DLS and TEM. Furthermore, these vesicles were readily absorbed by cells and fluorescence imaging of the cells could be achieved by uptake of either 1,6dihydroxynaphthalene or adamantylamine to break the ternary complex, releasing the 
fluorescent pyrene-peptide. Supramolecular polymeric analogues of these vesicles (Figure 33b) are pertinent for use as delivery devices of large biomolecules, in particular basic fibroblast growth factor (bFGF). ${ }^{318}$ These vesicles protected bFGF against several freezethaw cycles and were effective delivery devices to cells, therefore showing good promise as an injectable therapy. Das et al. expanded work in this area to form mixed micelles of viologen amphiphiles and tetralkylammonium salts in water. These micelles could

then be transformed into vesicles through host-guest complexation with CB[8]. ${ }^{319}$ Furthermore, the rearrangement of these mixid micelles into vesicles was mechanistically studied, appearing to progress through a stable mixed micelle stage; upon addition of $\mathrm{CB}[8]$, a dynamic mixture of non-spherical mixed micelles are formed, and then on addition of naphthalene derived guests, the micelles structurally promoted into self-sorted vesicles. $^{320}$

\subsubsection{Conclusion and outlook}

Supramolecular block copolymerization is an exciting area with ongoing development of facile routes towards producing multiply-responsive materials. From mechanistic studies of formation to advancement into bio-applicable delivery of growth factors, this area is undergoing new and extensive investigation. Supramolecular block copolymer materials are now poised for use in real-life applications and we hope to see in vivo progression in the near future.

\section{Molecular recognition in 3D supramolecular networks}

Hydrogels are becoming an important class of material on account of their high water content, tunable properties and biocompatibility. In the example of polymeric hydrogels, water is encapsulated within a chemically or physically crosslinked macroscopic network by capillary forces and their stiffness and elasticity defined by polymer loading 
and crosslink density. Hydrogels formed via molecular recognition of $\mathrm{CB}[n]$ for specific chemical motifs pendant from polymer chains have become increasingly widely studied since 2010. The fabrication of such soft materials has been reviewed specifically in recent years, perhaps however prematurely, as the field of $\mathrm{CB}[n]$ crosslinked hydrogels is still developing. ${ }^{133-135}$ In the following examples, exploiting the host-guest complexes of CB[6], $\mathrm{CB}[7]$ and $\mathrm{CB}[8]$ to form physical polymer-polymer crosslinks will be discussed and how the dynamics and control over such crosslinking has been investigated and utilized.

\section{1 $\mathrm{CB}[8]$ complexes crosslink guest functional polymers}

Scherman and coworkers have extensively explored the formation of CB[8] stabilized charge-transfer complexes between electron poor and electron rich species that are covalently bound to polymers. The random attachment of first and second guests, methyl viologen (MV) and naphthoxy (Np), respectively, to a variety of independent synthetic polymers was first achieved through the copolymerization of guest-bound monomers with either neutral, cationic or anionic monomers in a ratio of 1:10. ${ }^{321}$ Upon addition of $\mathrm{CB}[8]$ to a mixture of MV-polymer and Np-polymer ( $5 \mathrm{wt} . \%$ ) in water, viscoelastic materials were formed that expressed a responsiveness to stress and shear on account of the formation of multiple non-covalent ternary complexes between polymers, Figure 34 . Moreover, this work also demonstrates a wide tolerance of charge on the formation of hydrogels through the $\mathrm{CB}[8]$ recognition motif through the utility of a variety of anionic and cationic polymers, as well as the appearance of viscoelastically-tunable supramolecular networks through controlled $\mathrm{CB}[8]$ concentration.

Scherman et al. continued their exploration of these materials, focusing on the ability to form a predominantly elastic material with minimal polymer content $(0.5 \mathrm{wt} . \%) .{ }^{322}$ To achieve this a high molecular weight polysaccharide, hydroxyethyl cellulose (HEC, MW $=1.3 \mathrm{MDa}$ ), was functionalized with $\mathrm{Np}$ moieties to a degree of $5 \mathrm{~mol} \%$. The resulting material was then combined in solution with $\mathrm{CB}[8]$ and a high molecular weight com- 
a)

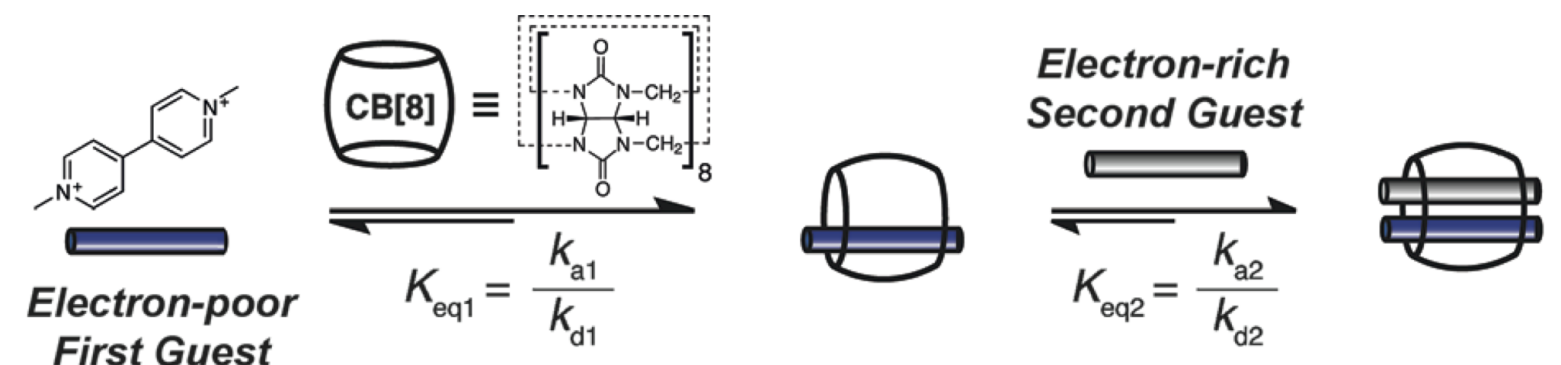

b)
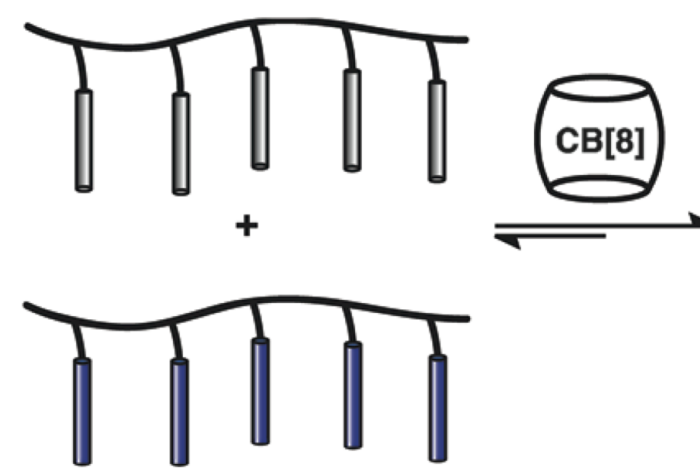

Multivalent Polymer Scaffold
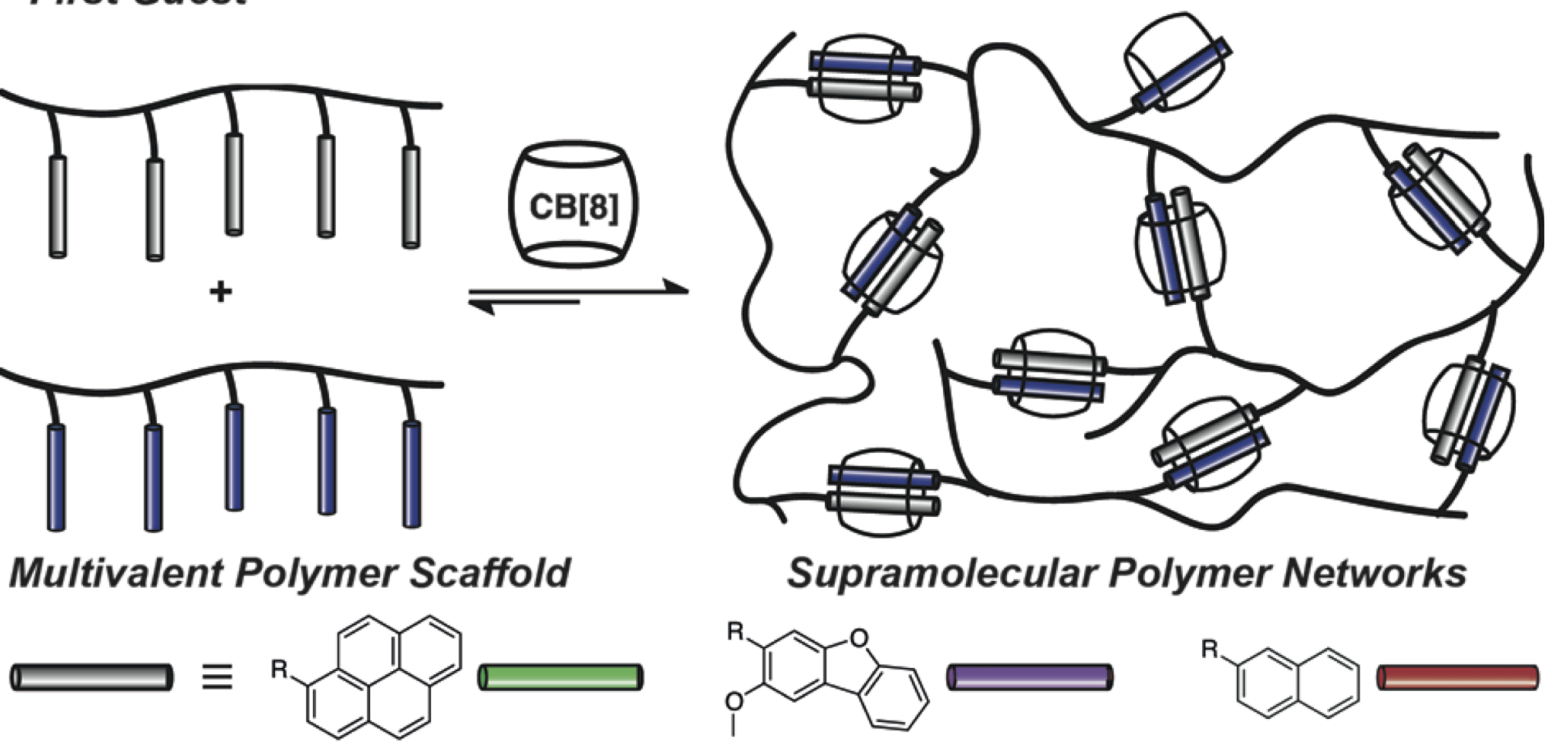

Supramolecular Polymer Networks<smiles>COc1cc2oc3ccccc3c2cc1P</smiles><smiles>Pc1ccc2ccccc2c1</smiles>

$\rightleftarrows$

Figure 34: Combination of multivalent polymers bearing first guest (MV) and second guest (either Pyr, DBF or Np) with $\mathrm{CB}$ [8] in water leads to supramolecular network formation and viscoelastic materials. Figure reproduced from Appel et al., Angew. Chem. Int. Ed., 2014 53, 10038-10042, with permission from John Wiley and Sons, Inc. 
modity polymer, polyvinyl alcohol (PVA, $\mathrm{M}_{n}=1.5 \mathrm{MDa}$ ) functionalized with MV. It was found that a range of soft material properties could be achieved and were accessible simply by modifying polymer loading. Impressively, a broad spectrum of storage moduli spanning two orders of magnitude $\left(10>G^{\prime}>1000\right)$ could be readily attained. Moreover, these materials were also shown to have excellent shear-thinning properties (resulting from the very low polymer loadings and non-covalent crosslinking) and also exhibited rapid self-healing behavior. The authors exemplified this by performing step-shear rheology experiments and demonstrated the viscosity of the material returned within seconds upon release of the high shear rates, and that the process was also recyclable. Rapid recognition of $\mathrm{Np}$-HEC by $\mathrm{CB}[8] \subset \mathrm{MV}-\mathrm{PVA}$ complexes upon relaxation of the material facilitates healing, whereby the dissociated complexes are quickly re-associated under mechanical stimuli rather than the actual breaking and making of any covalent bonds and so the properties are always $100 \%$ restored.

Furthermore, the release of small (lysozyme) and large (BSA) proteins from the HEC hydrogels has also been demonstrated. ${ }^{323}$ In this example, polymer concentration was altered in order to modulate the mesh size of the supramolecular network. By raising the polymer content, the mesh size was decreased and the proteins could be released at slower rates. Specifically, in the case of a $1.5 \mathrm{wt}$. \% HEC gel, BSA release was sustained for 160 days showing excellent utility for these materials as biomedical delivery devices.

Modification of the system to use the polysaccharride chitin afforded hydrogel materials that were antimicrobial, however, have found utility as a consolidant for archaeological wood conservation, specifically on the Mary Rose, a 16th Century warship. ${ }^{324}$ By adding extra $\mathrm{CB}[8]$ binding moieties onto the chitin backbone, such as naphthalene and dopamine derivatives, a system was produced that could not only gelate and express all previous properties of such supramolecular hydrogels, but also complex $\mathrm{Fe}^{3+}$ ions that persist in the water-logged artifacts. This system is therefore pulled into the porous wooden artifacts, strengthening them and 'locking' the iron in place preventing 
its transport and therefore further degradation, as well as providing biocidal protection.

Focussing on further improving $\mathrm{CB}[8]$ hydrogel utility in the biomedical field, harnessing 2:1 binding of amino acids to $\mathrm{CB}[8]$ was also investigated as a route to hydrogel formation. ${ }^{325}$ Scherman and coworkers demonstrated hydrogel formation with synthetic cationic styrenic polymers bearing either phenylalanine or tryptophan derivatives, Figure $35 \mathrm{~A}$. It was shown that phenylalanine 2:1 complexation to $\mathrm{CB}[8]$ afforded much stronger hydrogels than the tryptophan derived polymers, and that the material properties could be tuned by controlling $\mathrm{CB}[8]$ content and therefore crosslink density. This system is inherently simpler than the previous hydrogels as the network is only comprised of two components, a multivalent amino acid bearing polymer and $\mathrm{CB}[8]$, opposed to two different polymers (one bearing the first guest and the other the second guest) and $\mathrm{CB}[8]$. However, the authors found that rigid polymers were required to form hydrogels as this prevented intra-chain complexation of $\mathrm{CB}[8]$, as previously observed when polymers containing both first and second guest formed soft nanoparticles on addition of $\mathrm{CB}[8] \cdot{ }^{326} \mathrm{In}$ the future, the authors look to apply these amino acid recognition motifs to larger and more biomedically-relevant polymers to improve water content and biocompatibility further.

A second example of 2:1 guest to $\mathrm{CB}[8]$ binding facilitating hydrogel formation was reported by Lin et al. in 2013, Figure 35B. ${ }^{327}$ Here N-(4-dimethylaminobenzyl)chitosan (EBCS) was shown to form hydrogels of a variety of strengths upon addition of $\mathrm{CB}[8]$ under various $\mathrm{pH}$ conditions, allowing for control of release rates of the model drug cargo 5-fluorouracil. In this case, hydrogelation likely occurs through recognition and affinity of the protonated aminobenzyl groups by the ureido-carbonyl portals of $\mathrm{CB}[8]$. Due to protonation of the aminobenzyl groups being a necessity, weak hydrogels were formed at pH 6.8 and strengthened at lower pH levels. Therefore, the facility to use this material in biomedical application is quite restricted, however $\mathrm{pH}$ control over specific recognition of aminobenzyl derived moieties has been clearly demonstrated. 

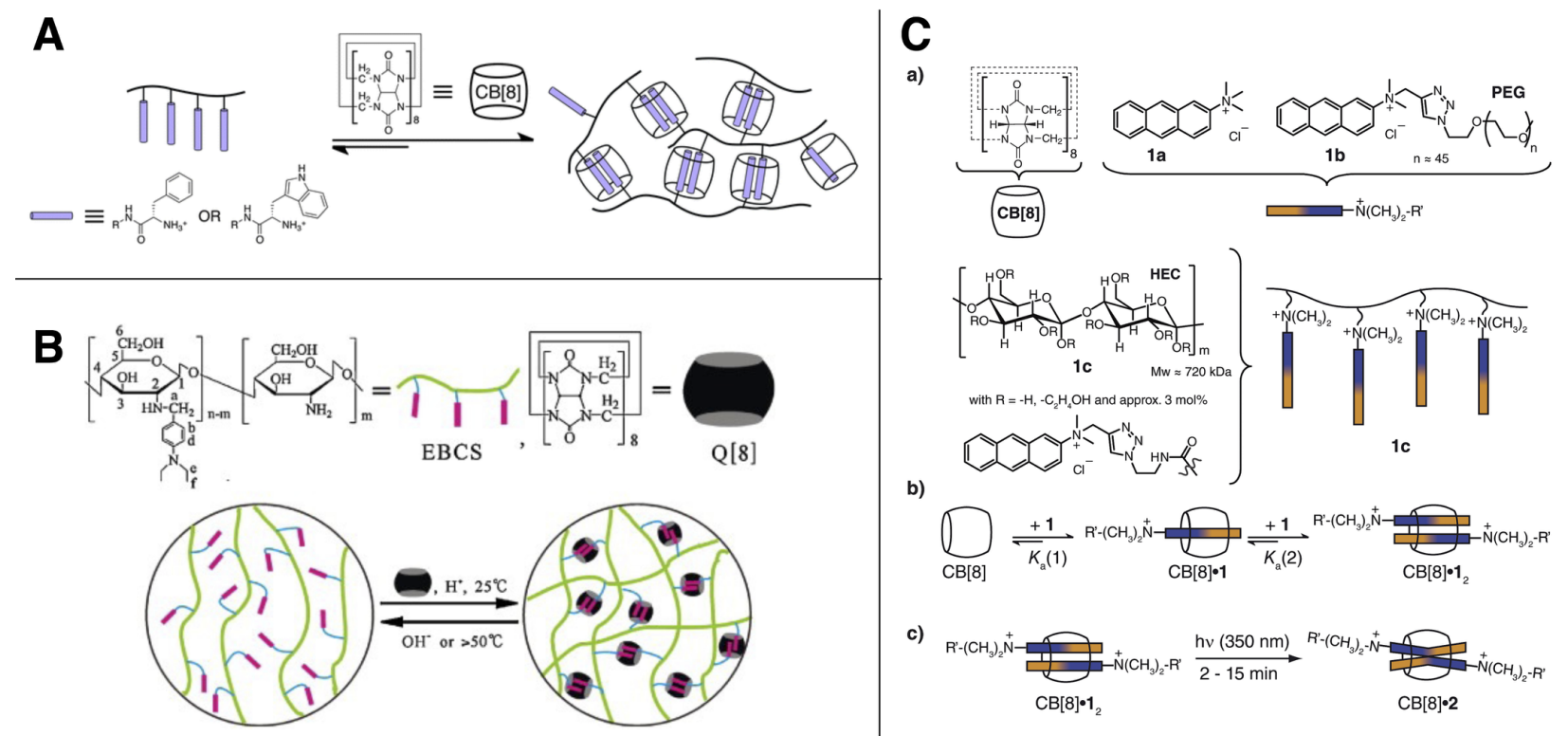

b)

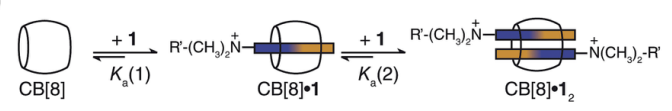

c) $\mathrm{R}^{\prime}-\left(\mathrm{CH}_{3}\right)_{2} \mathrm{~N}_{\mathrm{CB}[8] \cdot \mathbf{1}_{2}}^{+}$

Figure 35: Combination of multivalent polymers bearing residues that can bind in a 2:1 fashion with $\mathrm{CB}[8]$ (i.e. A: phenylalanine, tryptophan, B: N-4-diethylaminobenzene, C: anthracene) in water leads to supramolecular network formation. Figures adapted with permissions from A: Rowland et al., J. Mater. Chem. B, 2013, 1, 2904-2910, reproduced in part with permission from the Royal Society of Chemistry; B: Lin et al., Carbohyd. Polym. 2013, 92, 429-434, reproduced with permission from Elsevier Copyright 2013; C: Biedermann et al. Polym. Chem. 2014, 5, 5375-5382, reproduced in part with permission from the Royal Society of Chemistry. 
A further photo-responsive hydrogel construct from the Scherman group was reported in 2014. In this study, $\mathrm{CB}$ [8] catalyzed photodimerization of anthracene in water was exploited in hydrogel formation, Figure $35 \mathrm{C} .{ }^{45}$ In this system, the anthracene derived moieties form 2:1 inclusion complexes with $\mathrm{CB}[8]$ and were conjugated to an HEC backbone via a copper accelerated cycloaddition to azide derived HEC. The 2 anthracene : 1 CB[8] complexation alone afforded elastic soft materials. Upon photoirradiation at $350 \mathrm{~nm}$ for $15 \mathrm{~min}$ the material experienced a hardening effect and the moduli roughly tripled in magnitude, accounted for by the covalent dimerization of the two anthracene moieties within the $\mathrm{CB}[8]$, resulting in the HEC network becoming covalently crosslinked. The presence of $\mathrm{CB}[8]$ within the system was shown to rapidly catalyze the formation of the anthracene dimers as irradiation of the same material in the absence of $\mathrm{CB}[8]$ did not yield materials of the same strength. Unfortunately, the dimerization of anthracene in this system was not shown to be reversible so the hydrogelation via this photochemical $\mathrm{CB}[8]$ catalyzed crosslinking process is a seemingly permanent conversion.

\subsection{Dynamics of crosslinking influences material properties}

The nature of microscopic thermodynamic properties of physical crosslinks within a supramolecular network can have a large impact on the material. Recognition of different small molecule motifs by $\mathrm{CB}[8]$ in hydrogel formation thus have great effect on resulting hydrogel. In 2014, Scherman and coworkers explored the nature of the CB[8] ternary complex crosslinking motif and how the dynamic complexation of such motifs contribute towards defining hydrogel viscoelastic behavior. ${ }^{328}$ Investigation of different second guests beyond $\mathrm{Np}$ that have slightly different energetic binding properties to $\mathrm{CB}[8] \subset \mathrm{MV}$ was undertaken, Figure 34. The other second guests studied alongside Np were dibenzylfuran (DBF) and pyrene (Pyr), which have very similar overall equilibrium constants in the formation of the heteroternary complexes with $\mathrm{CB}[8] \cdot \mathrm{MV}\left(1.5,1.4\right.$ and $1.3 \times 10^{4} \mathrm{M}^{-1}$, respectively), determined by stopped-flow fluorescence experiments. Despite these seem- 
ingly similar equilibrium constants, the activation energies of dissociation $\left(E a_{d}\right)$ of the second guest were found to be vastly different (Np $30 \mathrm{~kJ} \mathrm{~mol}^{-1}$, DBF $54 \mathrm{~kJ} \mathrm{~mol}^{-1}$, Pyr $89 \mathrm{~kJ} \mathrm{~mol}^{-1}$ ). Physically crosslinked materials were prepared from multivalent poly(dimethylacrylamide) bearing one of the second guests, polystyrene-MV and CB[8]. Remarkably, all materials were found to have virtually identical X-ray scattering profiles, however, frequency-dependent oscillatory rheology showed that the Pyr gels were the "most elastic" and the Np gels the least. This was attributed to the dissociative rates of the second guest. The authors showed that when scaling the rheology data with the value of $E a_{d}$ for each second guest, the measured frequency-dependent rheology profiles overlayed almost exactly. Therefore, the strength and viscoelastic property of the hydrogels is not only dependent on conventional polymer physics and polymer interactions, but also dependent on the dynamics of crosslinking, in particular the choice of second guest used in the $\mathrm{CB}[8]$ ternary complex crosslinking motif, and specifically dependent on the $E a_{d}$ of the second guest. In simpler terms, higher dissociative barriers of the second guest affords longer crosslink lifetimes and therefore stronger materials.

In a subsequent study, Scherman and coworkers investigated how the dynamics of physical crosslinking in these systems affect release of rhodamine from the supramolecular network. ${ }^{329}$ Higher release constants were observed for materials bearing second guests with lower $E a_{d}$ values. This was accounted for through faster crosslink dynamics allowing faster diffusive rates of solvent and cargo in and out of the gel network. Faster crosslink dynamics also led to faster erosion rates of the hydrogels as water is able to diffuse into the hydrogel easier, facilitating erosion through swelling mechanisms. By studying these phenomena, erosive and diffusive mechanistic contributions to rhodamine release could be understood and material lifetimes are now potentially predictable. This study demonstrates that crosslink dynamics play a subtle yet important role in release of cargo from hydrogels, and that the ability to robustly fine tune release and erosive rates is possible. 


\subsection{Composite soft materials utilizing $\mathrm{CB}[8]$ host-guest interactions}

Collaboration between the Scherman and Ikkala groups resulted in the development of soft domain-hard domain composite hydrogels whereby homogeneous dispersions of cellulose nanocrystals (hard domains, CNCs) were crosslinked with soft polymeric domains via host-guest interactions between MV, Np and CB[8], Figure 36A. ${ }^{330}$ This was achieved by using CNCs decorated with surface ATRP initiator moieties to graft methacrylate polymers bearing multiple Np units. Mixing the functionalized CNCs with PVA-MV polymers and $\mathrm{CB}[8]$ produced supramolecular materials that were $85 \%$ water, extremely well structured as exemplified by frequency dependent rheology, highly elastic $\left(G^{\prime}>>G^{\prime \prime}\right)$ and rapidly self-healing (healing time $<6$ seconds). These are combined and desirable properties of both hard and soft materials which would usually be seen to be conflicting or unable to operate together in the same material. Moreover, materials that were cut in half and kept separate for 4 months were pushed back together and their viscoelastic property completely restored after just a few seconds. This property is remarkable in that the material was not passivated even after months and did not require any activation, something that had not been previously demonstrated in other supramolecular systems.

A followup study between the two groups led to the development of a composite hydrogel of nanofibrillar cellulose (NFC) and HEC, again supramolecularly crosslinked with $\mathrm{CB}[8]$ host-guest interactions. ${ }^{331}$ More specifically, a supramolecular HEC network, crosslinked by heteroternary complexation to $\mathrm{CB}[8]$ was adsorbed onto NFC colloidal domains as a sacrificial yet self-healing network, connecting stiffer NFC domains that would usually form stiff hydrogels alone through crystalline fibril-fibril entanglements, Figure 36B. These crystalline domains however have little ability to dissipate stress and as the materials are stiff, allow for very little yielding. By adsorbing a supramolecular network to bridge the length scales between NFC colloidal domains, a soft material was produced whereby the yield strain could be offset dependent on the supramolecular content of such formulations, and materials were also self healing. 

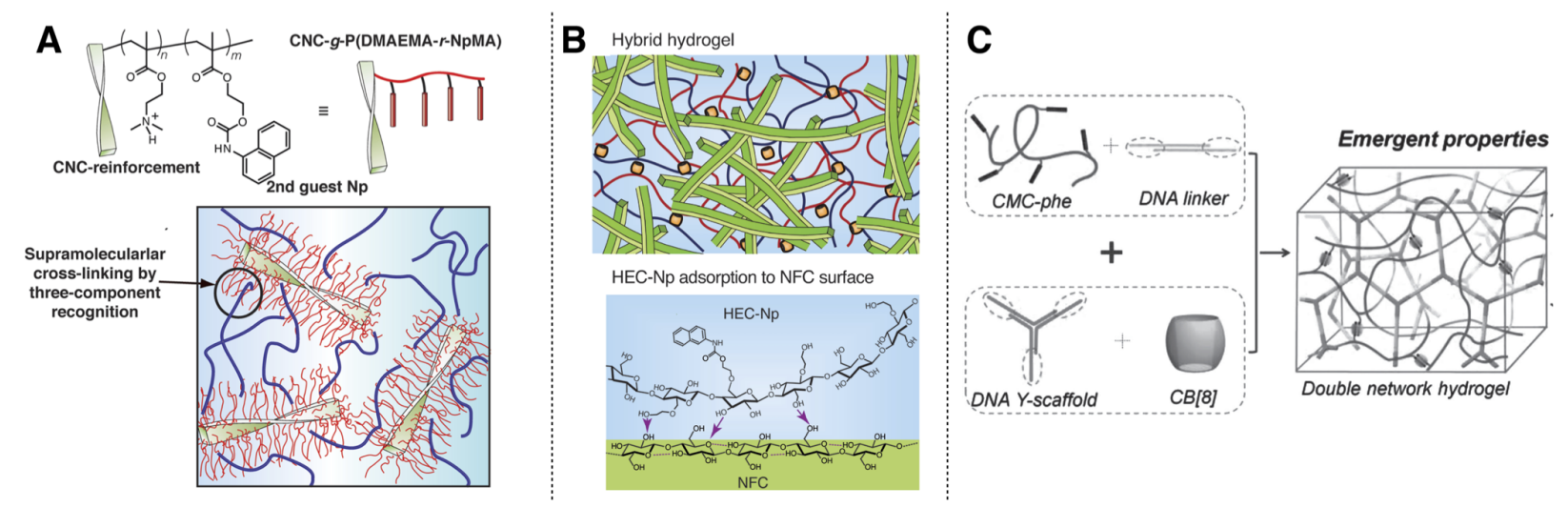

Figure 36: Examples of composite materials. A: CNC dispersions physically crosslinked to MV-polymers via CB[8]. B: Colloidal NFCs with length scales crossed by supramolecular hydrogel adsorption to offset stress. C: Purely supramolecular double networked hydrogel comprised of a DNA network and a cellulose/CB[8] network. All images reproduced in part with permissions from A: McKee et al. Adv. Funct. Mater. 2014 24, 2706-2713; B: Janecek et al. Angew. Chem. Int. Ed. 2015 54, 5383-5388, Copyright Wiley 2015; C: Li et al. Adv. Mater. 2015, 10.1002/adma.201501102, with permission from John Wiley and Sons, Inc.

Scherman, Liu and coworkers later reported a purely supramolecular double network hydrogel comprised of one DNA hybridized system and one $\mathrm{CB}[8]$ host-guest system, Figure 36C. ${ }^{332}$ This work is particularly interesting because of the multiple levels of stimuli responsiveness arising from the two present networks including temperature, chemical, mechanical and enzymatic responses. By mixing carboxymethyl cellulose-phe (CMCphe), $\mathrm{CB}[8]$ and DNA motifs, interpenetrating networks were afforded that did not appear to have any interaction as imaged by confocal microscopy, however, the brittleness of the DNA gel was offset by the presence of the interpenetrating CMC-phe/CB[8] dynamic system, which can rapidly reorganize and heal on distortion. Ultimately, a system was recognized that exhibited a higher than predicted mechanical strength of the combined gels and also the self-healing and rapid reorganization of $\mathrm{CB}[8]$ gels. The material was also shown to be responsive to excess free phenylalanine, which could compete for the $\mathrm{CB}[8]$ cavities, as well as having full biodegradability profiles on account of enzyme addition such as nuclease and cellulase. This material offers an interesting opportunity to consider new 3D cell culture scaffolds as well as injectable delivery devices for triggered 
release of DNA motifs in novel therapies.

\subsection{From monohydroxylated-CB $[n]$ to functional materials}

Kim et al. have reported preparation of $\mathrm{CB}[6]$ grafted hyaluronic acid (HA) via reaction of thiol-modified HA (HA-SH) with (allyloxy) ${ }_{12} \mathrm{CB}[6] .{ }^{333}$ Hydrogels were formed when HA functionalized with diaminohexane (DAH) was mixed with HA-CB[6] as a result of the specific inclusion complexation of the DAH to the CB[6] cavity. These hydrogels could be returned to their sol state upon addition of spermine to the material, which displaces the DAH moieties from the $\mathrm{CB}[6]$ cavities based on having a 100-fold higher binding constant, destroying the inter-chain crosslinks, suggesting gelation is promoted by $\mathrm{CB}[6] \cdot \mathrm{DAH}$ complexation. At 2 wt.\% polymer loading, these hydrogels exhibited exceptionally high storage moduli $(\sim 2 \mathrm{kPa})$ and also reasonable biocompatibility. Any remaining HA-DAH moieties were able to be capped with further $\mathrm{CB}[6]$ derivatives that were tagged with biorelevant molecules such as adhesion peptides and fluorescent markers demonstrating utility in 3D cell culture and tissue engineering.

These hydrogels were later implicated in controlling chondrogenesis of human mesenchymal stem cells (HMSCs). ${ }^{334}$ Here, spatial control of the HMSCs was achieved by modulating crosslink density of the hydrogel by controlling the amount of functionalization of HA with monoallyloxy-CB[6] and differentiation of the cells controlled by release profiles of transforming growth factors and dexamethasone bound $\mathrm{CB}[6]$ (Dexa-CB[6]). Dexa-CB[6] was shown to have slower release rates from the gels in comparison to free dexamethasone (in the order of days compared to hours) on its complexation to free DAH residues conjugated to the polymer network.

Similarly to Kim's work, promise of useful materials with multiple anchored CB[7]

molecules has also recently been reported by Tan and coworkers. ${ }^{105}$ In this work, the synthesis of a 4-vinylbenzyloxy-CB[7] monomer is reported followed by the copolymerization with $\mathrm{N}$-isopropylacrylamide (NiPAAm). The authors found that they could copoly- 
(a)

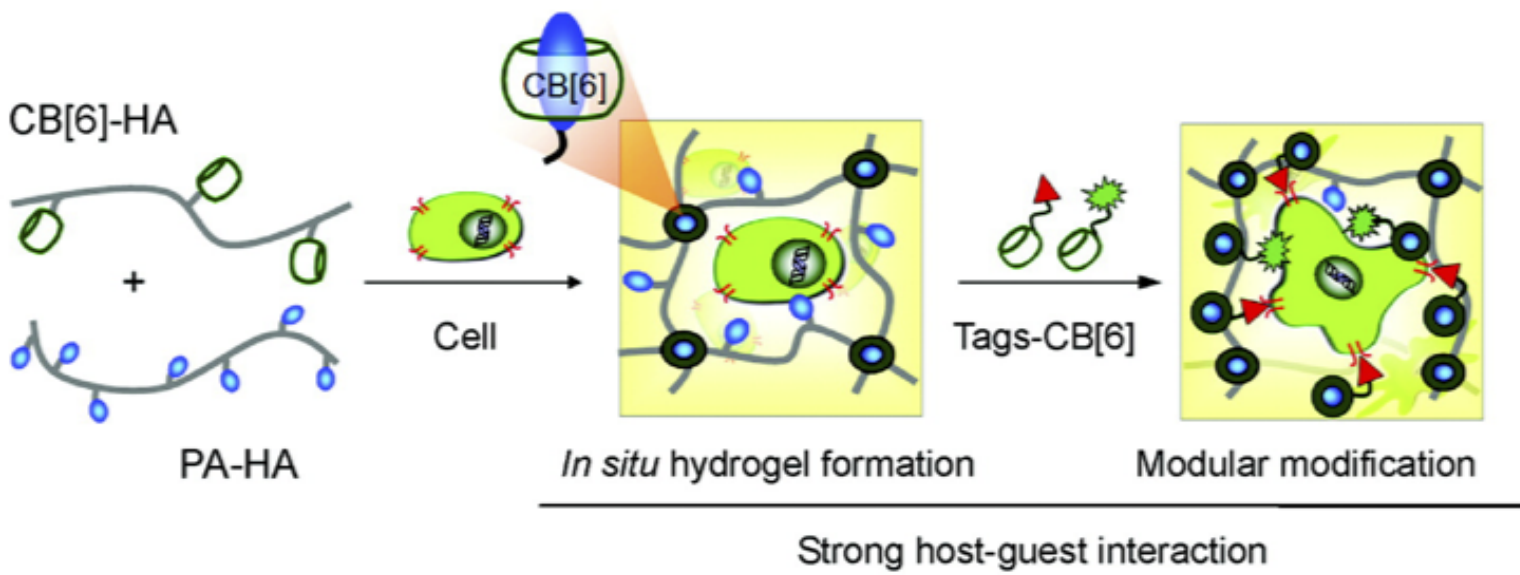

(b)

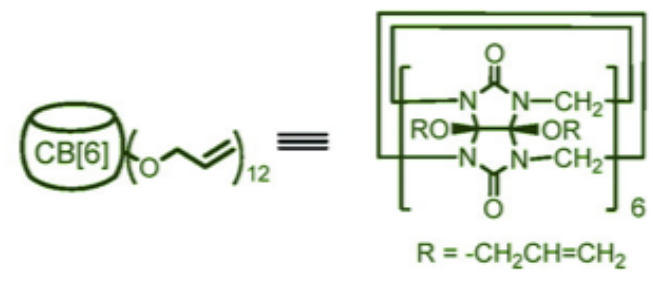

(c)<smiles>CNCCCCCNCCCNCCNCCN</smiles>

Figure 37: Attachment of $\mathrm{CB}[6]$ directly to hyaluronic acid and combination with PA-HA affords a good cell culture scaffold with further opportunity to complex cell-signalling moieties to the hydrogel network for tissue engineering applications. Figure reprinted from Park et al., ACS Nano, 2012, 6, 2960-2968. Copyright 2012 American Chemical Society 
merize $0.86 \mathrm{~mol} \%$ of 4-vinylbenzyloxy-CB[7] into NiPAAm using redox initiated polymerization in water with $M_{n}=2.10 \times 10^{5} \mathrm{Da}$ and PDI of 1.69 whilst retaining thermoresponsive properties. Although no specific materials were produced from this copolymer, a wide variety of guest molecules were still able to bind to a proportion of the CB[7] residues, even in their anchored form, and thus these materials could become useful in the construction of higher order supramolecular materials or in surface attachment applications.

\subsection{Microcapsule formation through $\mathrm{CB}[8]$ ternary complexation}

$\mathrm{CB}[8]$ recognition motifs have also found utility in the formation of synthetic microcapsules whereby complete control of the composition of the shell and also the content is paramount. Work by Scherman et al. saw development of multi-stimuli responsive polymer-gold nanoparticle composite materials, held together by $\mathrm{CB}[8]$ heteroternary complexes. ${ }^{335}$ This later developed, through collaboration between Scherman, Abell and coworkers, into composite gold nanoparticle-polymer microcapsules produced by microfluidic methods, Figure 38A. ${ }^{336}$ Similarly to the formation of supramolecular polymers and hydrogels, heteroternary complexation was achieved by encapsulation of an electron deficient first guest followed by complexation of an electron rich second-guest. To control structure formation, separate aqueous solutions of MV-functional gold nanoparticles, Np-copolymer and $\mathrm{CB}[8]$ were simultaneously injected into a microfluidic device with a fluorous-oil carrier phase perpendicular to the aqueous flow. As the oil and water phases meet, shear-forces at the interface initiate formation of monodisperse droplets whereby the Np-copolymer and MV-nanoparticles were crosslinked at the oil-water interface by $\mathrm{CB}[8]$ (having been drawn to the interface to stabilize the water/oil surface energy), forming spherical capsules upon dehydration, subsequent rehydration was carried out with minimal rupturing of the 3D structures. The size of the microcapsules could also be controlled by moderating the difference in flow rate between the oil carrier phase and the 
aqueous phases. These capsules were resistant to high temperatures and low pressures and no capsules were formed on replacing $\mathrm{CB}[8]$ with $\mathrm{CB}[7]$ as only binary complexes can be formed and therefore no crosslinking is achieved. Sustained encapsulation of FITC labelled dextran $(500 \mathrm{kDa})$ was also demonstrated, the integrity of the capsule shell was not compromised and the cargo distributed evenly throughout. Release of the cargo could even be triggered by reduction of the MV moieties with sodium dithionite, which breaks the $\mathrm{CB}[8]$ complexation causing dissolution of the capsule walls. Extra utility of the capsules arose from the presence of gold nanopaticles in the shell and this allowed for the analysis and/or detection of materials encapsulated within by surface enhanced Raman spectroscopy (SERS) as well as mapping of the microcapsule surface.

In a followup communication, Abell and coworkers demonstrated the fabrication of colloidosomes via analogous microfluidic methods, Figure 38B. ${ }^{337}$ Passive diffusive release of cargo from colloidosomes is highly dependent on pore sizes, which is determined by the spacing between colloids. Therefore, the ability to control distances between colloids in microcapsule walls, as well as anchoring the colloids in place, is extremely desirable. Similarly to the production of the aforementioned gold nanoparticle-polymer composite capsules, polystyrene colloids were functionalized with MV and then mixed with $\mathrm{CB}[8]$ and a copolymer bearing $\mathrm{Np}$ moieties, forming capsules, which were stable upon dehydration. Encapsulation and passive release of various molecular weight FITCdextran was explored and the authors demonstrated that $150 \mathrm{kDa}$ FITC-Dextran could be encapsulated for a prolonged time period as a results of the small gap distance between colloids. Release could also be triggered by addition of a competitive guest (adamantyl amine, ADA) by disassembly of the crosslinks and therefore the capsule. The authors hypothesize that the release rates could be altered by controlling the inter-colloid distance and this could be achieved by changing polymer concentrations, crosslink density or parameters of the microfluidic device.

The latest example of microcapsule formation facilitated by the efficient recognition 


\section{A: polymer/nanoparticle composite capsules}

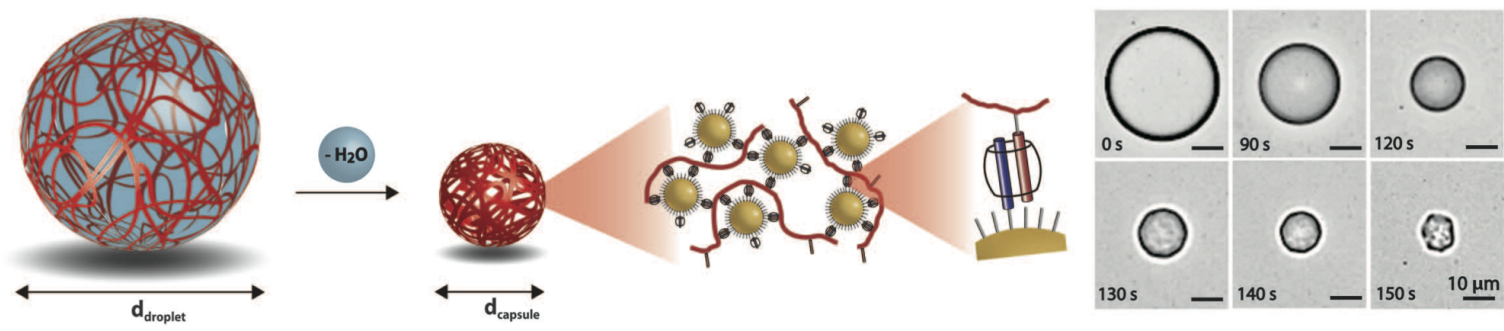

\section{B: colloidosomes}

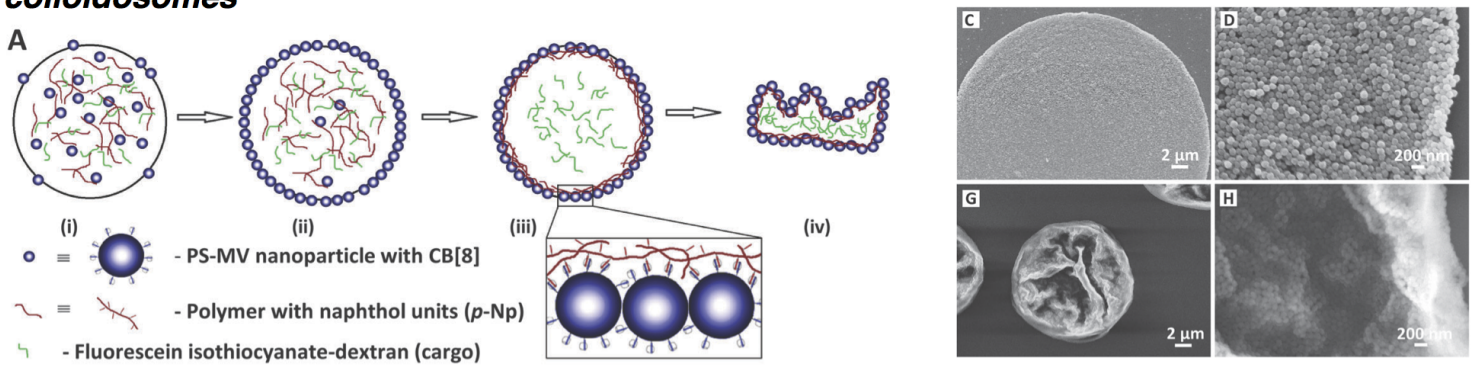

\section{C: layered assembly of polymer capsules}
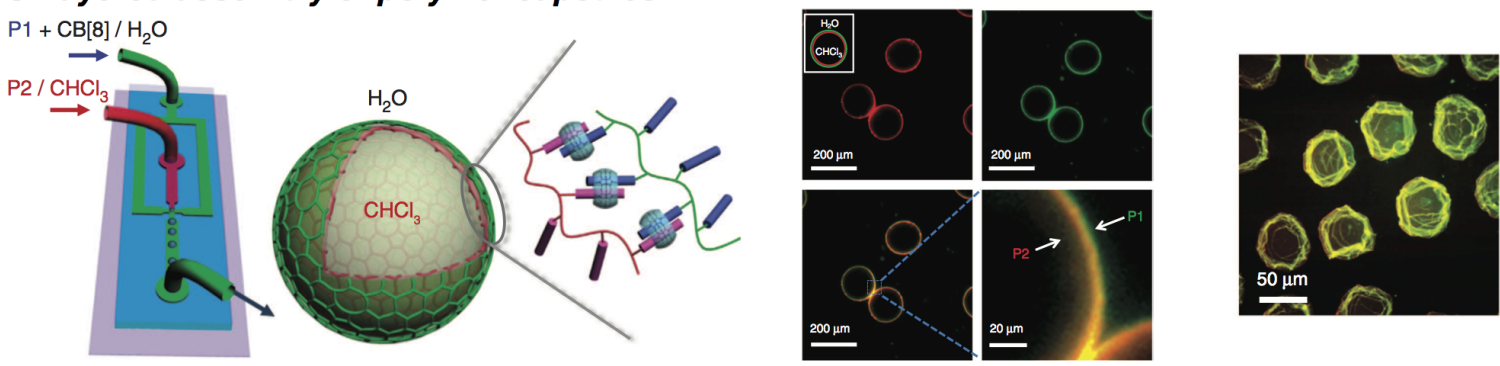

Figure 38: Microfluidic methods for supramolecular capsule formation. A: CB[8] hostguest interactions between gold nanoparticles and multivalent guest polymers provide entanglements strong enough to build microcapsule walls. B: Colloids cross linked with $\mathrm{CB}[8]$ into polymeric microcapsule walls, controlling inter-colloid distance alters release rates of encapsulated cargos. C: Layered assembly of polymer only microcapsules by dissolving multivalent guest polymers in both aqueous and non-aqueous phases of the microfluidic device whereby $\mathrm{CB}$ [8] facilitates complexation at the oil-water interface. Figures reproduced with permissions from A; Zhang et al. Science, 2012, 335, 690-694, Copyright AAAS 2012; B: Stephenson e al. Chem. Commun. 2014, 50, 7048-7051, reproduced with permission from The Royal Society of Chemistry; C: Zheng et al. Nat. Commun. 2014, 5, 5772, Copyright NPG 2014. 
of molecular moieties by $\mathrm{CB}[8]$ under microfluidic conditions was shown by Scherman et al. in $2014 .{ }^{338}$ Assembly of multiple polymer layers and crosslinking with CB[8] was achieved at a water/chloroform interface allowing for microcapsule formation. In this system there is no requirement for nanoparticles to direct assembly at the interface as guest-bearing copolymers were applied to both the aqueous (MV-polymer) and oil (Nppolymer) phases to template capsule formation along the oil/water interface and layered assembly was demonstrated by confocal microscopy of rhodamine and fluorescein labelled components, Figure 38C.

Adding extra functionality to the microcapsules, the hydrophobic polymer could be replaced with a branched dendritic polymer bearing azobenzene moieties. Incorporation of the branched dendritic polymer system allowed small molecules to be encapsulated within the microcapsules and the skin formation facilitated through 1:1:1 complexation of $\mathrm{CB}[8], \mathrm{MV}$ and azobenzene. Disassembly could be triggered by UV irradiation, switching the azobenzene configuration from $\mathrm{E}$ to $\mathrm{Z}$ thereby breaking the heteroternary complexes, facilitating cargo release. These systems have demonstrated robust assembly techniques, excellent tuning capabilities and therefore have many potential applications in future delivery devices.

\subsection{CB $[n]$ crosslinked polymer networks outlook}

$\mathrm{CB}[n]$, and specifically $\mathrm{CB}[8]$, have shown excellent utility in the development and formation of novel materials. Robust and efficient recognition motifs allow for rapid assembly of macroscopic polymer networks of which the dynamics of such ternary complexes have been shown to have impact on material properties. In the example of supramolecular hydrogels, the mere fact that polymer concentration can be retained and yet a series of different material properties can be accessed by simply modulating $\mathrm{CB}[8]$ concentration and therefore crosslink density allows for a wide applicability of these hydrogels to future technologies, e.g. 3D cell culture. This is of particular importance in biomedical 
applications whereby fast-healing materials are required as injectables, $\mathrm{CB}[8]$ crosslinked hydrogels would be highly impactful on modern medicines. Manipulation of polymeric materials by microfluidic devices has led to novel microcapsule structures with excellent control over assembly processes and potentially excellent utility in future molecular manipulation and delivery systems.

However, the science of covalently attaching $\mathrm{CB}[8]$ to polymers has yet to be reported, but is envisaged to lead to the development of extremely responsive multi-component materials with fantastic tuning properties. Therefore, in the future we hope and expect to see many efforts in reproducible and scalable methods to mono-functionalize $\mathrm{CB}[8]$ with readily reactive groups and covalently attach such a molecule to polymer architectures.

\section{Catalysis within Cucurbituril Cavities}

The ability of $\mathrm{CB}[n]$ s to encapsulate various guest molecules has led to them being used to catalyze and template various chemical reactions. The first report detailed the catalysis of [3+2]-cycloadditions ${ }^{339}$ in the presence of $\mathrm{CB}[6]$, which paved the way for the development of click-chemistry reactions. Since then, $\mathrm{CB}[n]$ s have been used to catalyse solvolysis reactions, ${ }^{340}$ oxidation reactions ${ }^{341}$ and metal-ion assisted reactions. ${ }^{342}$ Furthermore, $\mathrm{CB}[n] \mathrm{s}$ have been utilized in photocatalysis and templating photochemical reactions, ${ }^{343}$ and, in contrast, have been employed in reaction inhibition through the protection of reactive functional species by encapsulation. ${ }^{344}$ The following section will focus on key research outcomes in the area of catalysis and reaction modulation through interactions with $\mathrm{CB}[n]$ macrocycles.

\section{$11.1[3+2]-c y c l o a d d i t i o n s$ catalysed by $\mathrm{CB}[6]$}

The first instance of CB catalysis was reported by Mock et al. in the late 1980s, whereby a [3+2]-cycloaddition of an alkyene (E1) and an azide (E2) was shown to occur within the 
cavity of $\mathrm{CB}[6]$ molecules. ${ }^{339}$ In an aqueous formic acid solution at $40{ }^{\circ} \mathrm{C}$, the cycloaddition occurs slowly with the formation of substituted triazoles, however the addition of catalytic amounts of $\mathrm{CB}[6]$ led to a $6 \times 10^{5}$ increase in reaction rate with triazole (E3) being the only isomer formed as result of the orientation of (E1) and (E2) within the 1:1:1 complex formed with $\mathrm{CB}[6]^{339}$ (see Figure 39). The catalytic rate increase of the reaction was attributed to an overcoming of entropic constraints through optimal orientation, in addition to strain-induced compression of the reactants within the $\mathrm{CB}[6]$ cavity. This pioneering work inspired the development of azide-alkyene cycloadditions toward 'click chemistry' reactions, with rotaxanes, polyrotaxanes and pseudorotaxanes being successfully synthesized from CB[6] catalyzed [3+2] cycloadditions. ${ }^{345-349}$

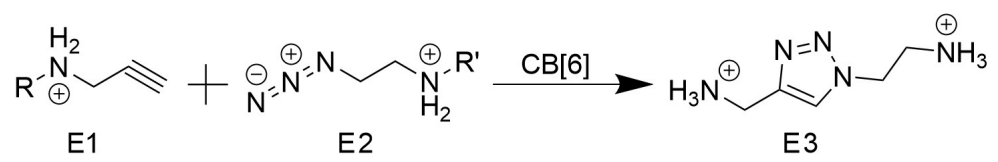

Figure 39: A schematic of an azide-alkyne click reaction mediated by $\mathrm{CB}[6] .{ }^{339}$

\section{2 $\mathrm{CB}[n]$ catalysed solvolysis reactions}

In 2009, Nau et al. reported the catalysis of amides, carbamates and oximes by CB[6] and $\mathrm{CB}[7]$ through an acid hydrolysis reaction. ${ }^{340}$ In the presence of $\mathrm{CB}[6]$ or $\mathrm{CB}[7]$, the rate of hydrolysis of an amide with a cadaverine moiety $(\mathbf{E} 4)$ rose by a factor of $5\left(k_{\text {cat }} / k_{\text {uncat }}\right)$ at $\mathrm{pD} 0.9$, whilst at $\mathrm{pD} 1.4$ the reaction rate increased by a factor of 11.6. For carbamate (E5), reaction rate increased by a factor of approximately 30 in the presence of $\mathrm{CB}[6]$. For oxime (E6) in the presence of $\mathrm{CB}[7]$, the reaction rate increased by factors of between 50 and 285 dependent on the $\mathrm{pD}$ value, which was varied between 4 and 5.8. The catalytic effect of CBs was proposed to be through the coloumbic stabilization of the protonated nitrogen intermediate that occurs during the hydrolysis, in addition to increasing their apparent $p K_{\mathrm{a}}$ values.

Garcia-Rio et al. investigated the hydrolysis of substituted benzoyl chlorides (E7) in 
(a)
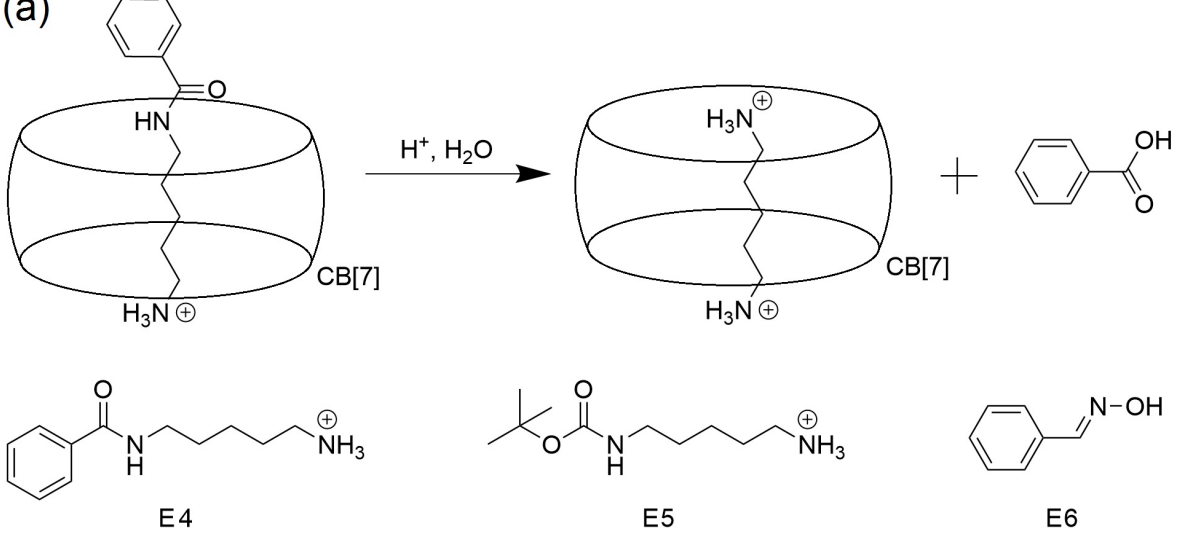

(b)

$$
\mathrm{X}=4-\mathrm{CH}_{3} \mathrm{O}
$$$$
\mathrm{O} \sim_{\mathrm{Cl}}
$$$$
\text { S }
$$

E7
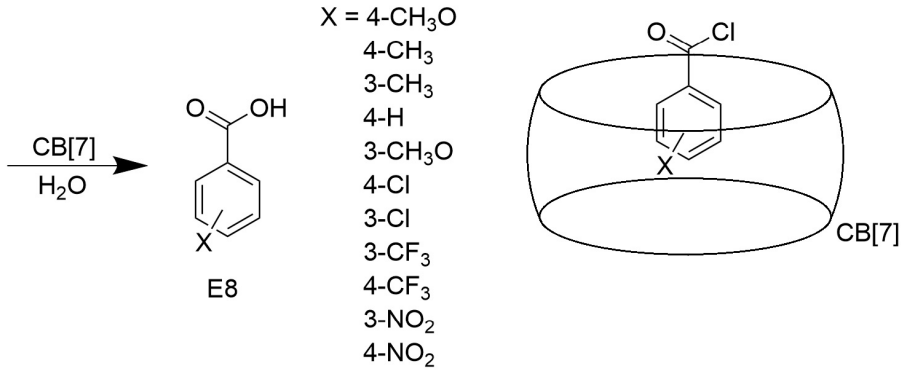

Figure 40: (a) Acid hydrolysis of an amide (E4), a carbamate (E5) and an oxime (E6) catalyzed by $\mathrm{CB}[7]{ }^{340}$ (b) $\mathrm{CB}[n]$ mediated hydrolysis of benzoyl chlorides to benzoic acid. ${ }^{350}$

the presence of either $\mathrm{CB}[7]$ or methylated- $\beta$-cyclodextrin, with each macrocycle displaying contrasting effects on the reaction. ${ }^{350}$ For the case of benzoyl chlorides substituted with electron donating groups, $\mathrm{CB}[7]$ showed a catalytic effect towards the formation of benzoic acid (E8), whereas methylated- $\beta$-cyclodextrin inhibited the reaction. Conversely, electron-withdrawing substituted benzoyl chlorides were inhibited by $\mathrm{CB}[7]$ and catalyzed by methylated- $\beta$-cyclodextrin. The opposing effects are explained through differences in the cavities and portals of these two macrocycles. For electron donating substituted benzoyl chlorides, $\mathrm{CB}[7]$ catalyzes the reaction through stabilization of the acylium cation intermediate by its carbonyl lined portals, lowering the energy barrier for the reaction. For the electron withdrawing substituted benzoyl chlorides, methylated$\beta$-cyclodextrin shows a catalytic effect as a result of the hydroxyl groups of the portals participating in the reaction. This work demonstrated the role that macrocycle portals can play influencing reaction rates. 


\section{3 $\mathrm{CB}[n]$ catalyzed oxidation reactions}

In 2009, Zhu et al. demonstrated the ability of $\mathrm{CB}$ [8] to catalyze the oxidation of veratryl

alchohol to veratraldehyde by 0 -iodoxybenzoic acid, ${ }^{341}$ and then extended the approach to the oxidation of aryl, allyl and alkyl alcohol compounds. ${ }^{351}$ The catalysis was found to be highly dependent on temperature and moderately dependent on $\mathrm{pH}$, with neutrality favored over more extreme $\mathrm{pH}$ levels. Although the role of $\mathrm{CB}[8]$ in the catalysis was not clear, an increase in the oxidative conversion of $16.3 \%$ was observed at $95{ }^{\circ} \mathrm{C}$ in the presence of $\mathrm{CB}[8]$.

Reddy et al. investigated the catalysis of a series of reactions involving halogen inclusion complexes with $\mathrm{CB}[6] .{ }^{81}$ Fine $\mathrm{CB}[6]$ powders were exposed to either $\mathrm{I}_{2}$ or $\mathrm{Br}_{2}$ vapor to form $\mathrm{I}_{2}-\mathrm{CB}[6] \cdot 4 \mathrm{H}_{2} \mathrm{O}$ and $\mathrm{Br}_{2}-\mathrm{CB}[6] \cdot 10 \mathrm{H}_{2} \mathrm{O}$, respectively. Reactions were then performed in the presence of the halogen complex, or its complex with $\mathrm{CB}[6]$, with resulting yields being compared. The greatest increase in yield was seen for that of the iodine-catalyzed Prins cyclization, where the yield was $7 \%$ higher than that reported in the literature. The remaining reactions tested all showed yields comparable to what had been demonstrated in the literature for the the uncomplexed halogens. Nevertheless, this represents a novel use of $\mathrm{CB}[6]$ complexes in halogen-catalyzed reactions.

\subsection{Photocatalysis}

The efficiency of photochemical reactions can suffer when performed in solvated environments and where specific molecular orientations and alignments of reactants are required. In order to proceed effectively, such barriers need to be overcome. Molecules that have been excited with photons can relax either radiatively or non-radiatively (via solvent collisions etc.), which can drastically reduce reaction efficiency. In addition, the lack of control over molecule orientation can lead to many products arising from a single photochemical reaction, or indeed a reaction may not proceed at all if a particular molecular orienta- 
tion of the reactants is not met. The use of $\mathrm{CB}[n]$ macrocycles in photochemical reactions may help to overcome these obstacles. $\mathrm{CB}[n]$ s create a reaction environment that can be solvent free, preventing non-radiative relaxation. Also, $\mathrm{CB}[n] \mathrm{s}$ allow for precise orientation of guest molecules, allowing for specific stereo-selectivity of the reaction product. Lastly, $\mathrm{CB}[n] \mathrm{s}$ are transparent in the near $\mathrm{UV}$ and visible regions of the electromagnetic spectrum, promoting photochemical processes within the $\mathrm{CB}[n]$ internal cavity.

Kim et al. demonstrated the first use of $\mathrm{CB}$ in the catalysis of a photochemical reaction involving (E)-diaminostilbene (E9). ${ }^{343}$ 2:1 complexes of stilbene:CB[8] were shown to form in an aqueous environment as confirmed by ${ }^{1} \mathrm{H}$ NMR. The ternary complex then underwent a cycloaddition after UV irradiation $(300 \mathrm{~nm}, 0.5 \mathrm{~h})$, to form a [2+2] syn-adduct (E10) within the $\mathrm{CB}[8]$ cavity with high selectivity over the anti-adduct (E11). The same experiment performed in the absence of $\mathrm{CB}[8]$ yielded the $(\mathrm{Z})$-isomer (E12) as the major product; a product that was not evident at all when $\mathrm{CB}[8]$ was present. It was also demonstrated that the addition of base $(\mathrm{NaOH})$ to the post-photoreaction complex saw the ejection of the cyclyzed compound from the $\mathrm{CB}[8]$ cavity, regenerating the cucurbituril catalyst as a result. This work highlighted the use of $\mathrm{CB}[8]$ as a template nanoreactor that can influence regio- and stereoselectivity in photochemical reactions.

Continuing on from the work of Kim et al., Ramamurthy et al. explored the use of $\mathrm{CB}[8]$ in controlling the photoreactivity of a range of olefin molecules (some of which include E13, E14 and E15). ${ }^{352,353}$ The olefins were irradiated with UV light for $1 \mathrm{~h}$, in both the presence and absence of $\mathrm{CB}[6]$ and $\mathrm{CB}[8]$. In the presence of $\mathrm{CB}[7]$, compounds E13, E14 and E15 formed their own structural variations on the (Z)-isomer (E18) and the bridged (Z)-isomer (E19), with compound E5 also yielding the hydrolysis compound (E20). With $\mathrm{CB}[8]$ present, photodimerization was favored, with the product distribution favoring the syn-adduct (E16), with minor amounts of the anti-adduct (E17) and the bridged and non-bridged $(Z)$-isomers also present. This reaction scheme was then extended to the formation of symmetric and asymmetric cyclized dimers by localizing asymmetric olefins 

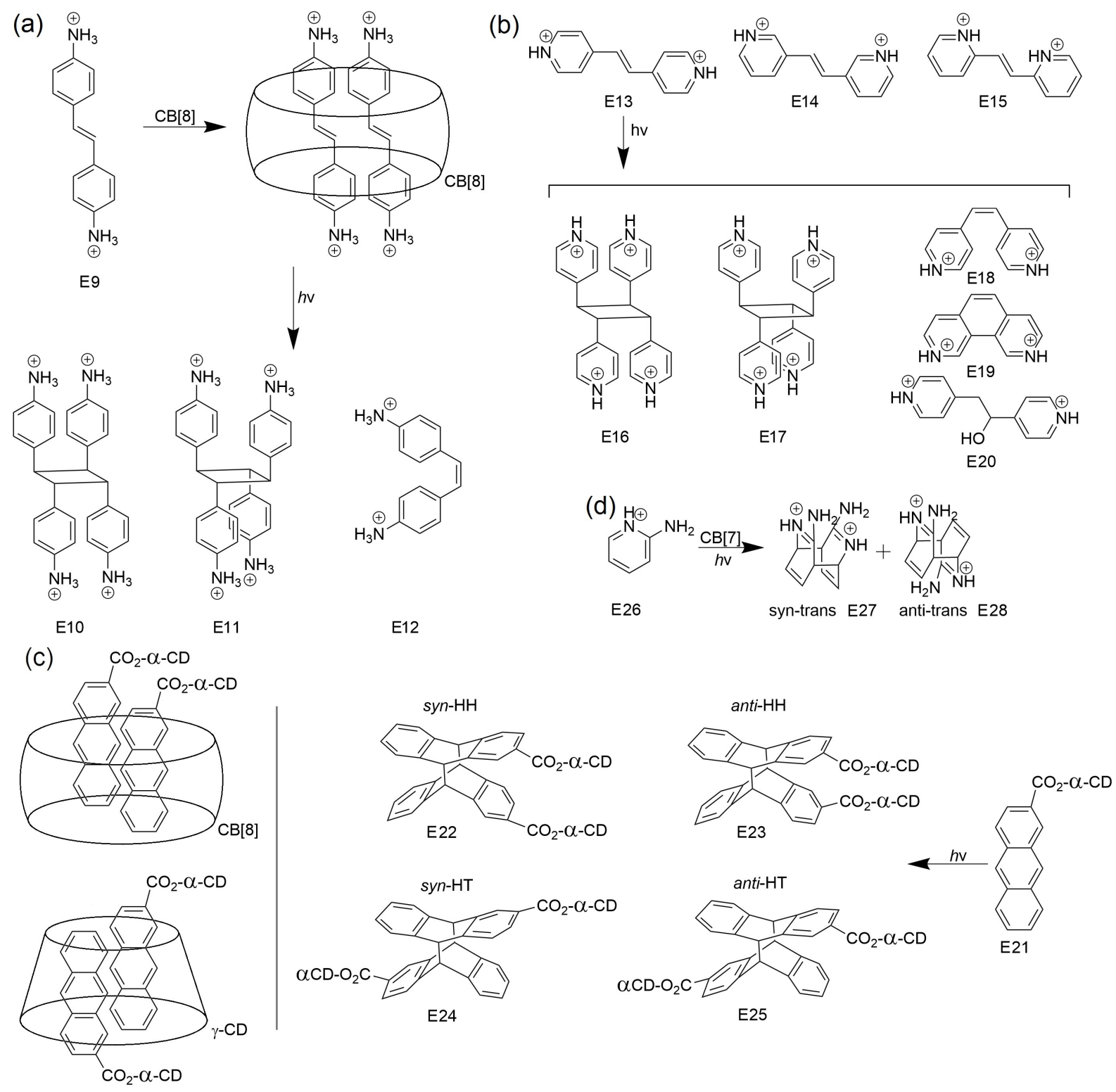

Figure 41: (a) Photodimerization of (E)-diaminostilbene in the presence of $\mathrm{CB}[8]{ }^{343}(\mathrm{~b})$ Photoreactivity of trans-1,2-bis(n-pyridyl)-ethylenes and stilbazoles in the presence of $\mathrm{CB}[8] .{ }^{352,353}$ (c) Photodimerization of cyclodextrin functionalized anthracene in the presence of $\mathrm{CB}[8]$ and $\gamma$-cyclodextrin. ${ }^{136}$ (d) Photodimerization of aminopyridine derivates encapsulated in $\mathrm{CB}[7] .{ }^{131}$ 
within the $C B[8]$ cavity. ${ }^{353}$

In an effort to expand the versatility of $\mathrm{CB}[8]$ in enhanced photoreactions, Pemberton et al. reported the photodimerization of neutral and cationic coumarin species. ${ }^{20,354}$ Irradiation of an aqueous solution of a coumarin and $\mathrm{CB}[8]$ in a 2:1 complex led to the formation of the head-to-tail adduct as the major product, a result that was independent on the functional group substitution of the coumarin derivatives used. The type of head-to-tail adduct (whether syn- or anti-) could be controlled by varying the substituents on the coumarin moiety, with polar substituents favoring the anti-adduct, and non-polar substituents favouring the syn-adduct.

Kim and Inoue et al. moved away from [2+2] cycloadditions to concentrate on [4+4] cycloadditions in the presence of both $\mathrm{CB}[8]$ and $\gamma$-cyclodextrin (CD). ${ }^{136}$ Both anthracene, and anthracene tethered to $\alpha-C D$ via an ester linkage (E21), were investigated as photoactive compounds to demonstrate that the stereoselectivity of the [4+4] cycloaddition can be manipulated via conditions outside the host cavity. Photoexcitation in the absence of $\mathrm{CB}[8]$ or $\gamma$-CD led to a mixture of syn-head-to-head (HH, (E22)), anti-HH (E23), syn-headto-tail (HT, (E24)) and anti-HT (E25) isomers forming in roughly the same ratios (with the HT isomers being favoured) for both anthracene and anthracene- $\alpha$-CD. The presence of $\mathrm{CB}[8]$ or $\gamma-\mathrm{CD}$ during photodimerization of anthracene led to a minor shift toward the formation of HT isomers. Photodimerization of anthracene- $\alpha-C D$ in the presence of $\gamma-C D$ showed a larger shift towards the HT isomers, with a ratio as high as 98:2 HT:HH at $-20^{\circ} \mathrm{C}$ and $210 \mathrm{MPa}$. The introduction of $\mathrm{CB}[8]$ into the anthracene- $\alpha-\mathrm{CD}$ system gave rise to a HT:HH ratio of 2:98; an extreme inversion compared to that of the $\gamma$-CD system, showing that the $\alpha-\mathrm{CD}$ attached to the anthracene, which is too large to be incorporated into the cavities of either $\gamma-\mathrm{CD}$ or $\mathrm{CB}[8]$, can drastically effect the stereochemistry of the products formed by photocycloaddition. More recently, the photodimerization of anthracene in $\mathrm{CB}[8]$ has been utilized by Biedermann et al. in the ligation and cross linking of polymer networks. ${ }^{162}$ 
Whilst $\mathrm{CB}[8]$ has also been applied to the stereo-control of photochemical reactions involving styryl dyes, ${ }^{138}$ cinnamic acids, ${ }^{355}$ 2-naphthalenecarbonitrile and naphthoate compounds towards the formation of cubane-like photodimers, ${ }^{137,356}$ as well as the photothydrolysis of benzoimidazole, ${ }^{357} \mathrm{CB}[7]$ has been shown to accommodate smaller, yet similarly designed guests for photodimerization. Macartney et al. investigated the [4+4] photodimerization of 2-aminopyridine (E26) when encapsulated in $\mathrm{CB}[7]$ in a 1:2 hostguest complex. ${ }^{131}$ In the absence of CB[7], a mixture of the syn-trans (E27) and anti-trans (E28) photodimers formed in a 1:4 ratio. In the presence of $50 \mathrm{~mol} \% \mathrm{CB}[7]$, the anti-trans dimer was formed exclusively. It was also found that $\mathrm{CB}$ [7] stabilized the dimer at room temperature, preventing it from reverting back to the monomeric 2-aminopyridine.

\subsection{Metal cation assisted $\mathrm{CB}[n]$ catalysis}

Given their negatively charged carbonyl fringed portals, cucurbiturils are highly efficient at binding to metal cations; a property that makes them useful at promoting catalysis reactions that utilize ionic metal species. Despite cation binding to $\mathrm{CB}[n] \mathrm{s}$ being a wellestablished area of research, ${ }^{36}$ the idea of using such complexes for catalysis is relatively new, and hence fairly unexplored. Demets et al. investigated the catalytic properties of an oxovanadium(IV)-CB[6] complex towards the oxidation of various organic compounds. ${ }^{358}$ It was demonstrated that pentane (E29), a linear alkane, could be oxidized to compounds E30, E31 and E32, however, larger organic molecules such as styrene (E33) and cyclohexane (E34) could not. This was explained through the nature of the $\mathrm{VO}_{2}{ }^{+}$ binding, whereby the vanadium atom bound to the carbonyl portal of the $\mathrm{CB}[6]$, with the oxygen atom pointing away from the $\mathrm{CB}[6]$ cavity, in a distorted square-pyramidal configuration. This meant that only small molecules that can enter the CB[6] cavity could be oxidized as the oxygen atom in the $\mathrm{VO}_{2}{ }^{+}$species blocked direct access to the vanadium atom from the other side of the complex. In this way, Demets et al. demonstrated a size selective heterogeneous catalyst based on $\mathrm{CB}[6]$ through metal ion interactions. 
(a)
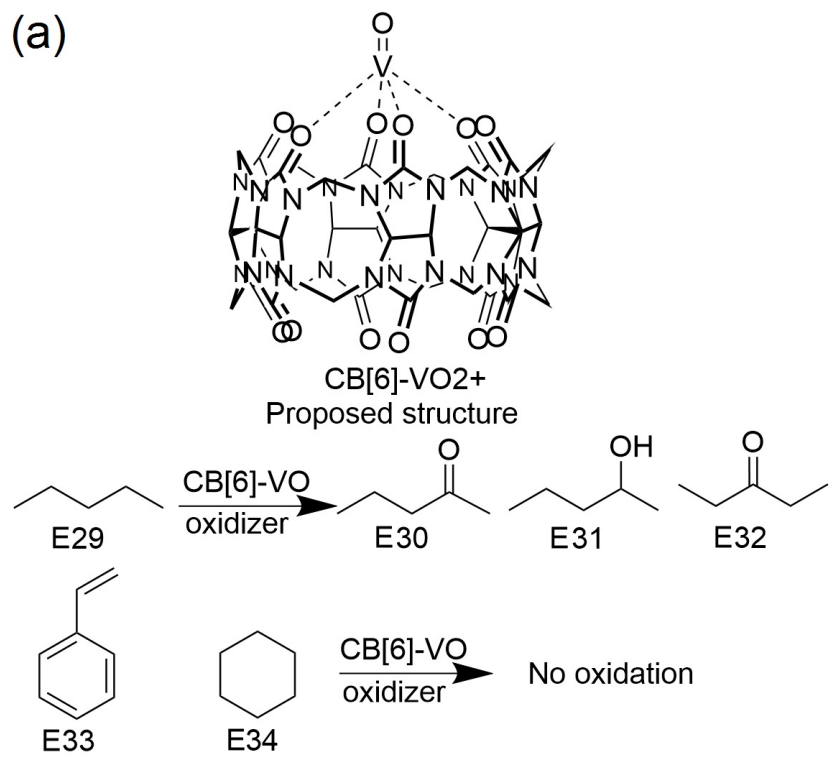

(b)

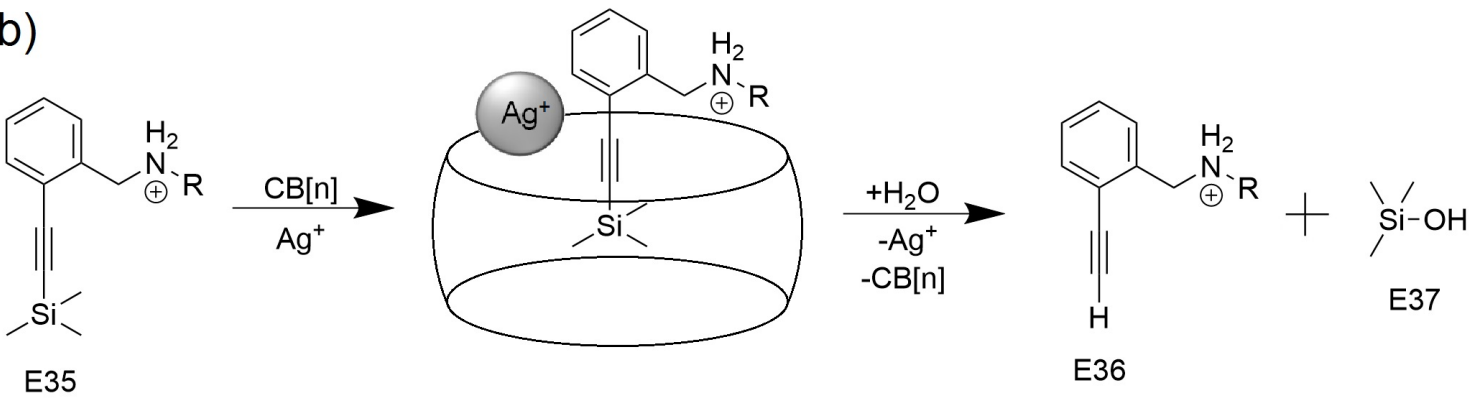

(c)

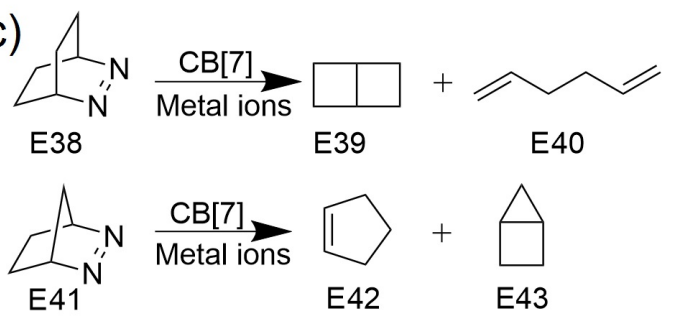

Figure 42: (a) Size selective oxidation of alkanes within a oxovanadium(IV)-CB[6] complex. Adapted from Demets et al. ${ }^{358}$ (b) Desilylation of trimethoxysilane compounds, catalyzed by silver ions in the presence of cucurbiturils. ${ }^{342}$ (c) Photoreactivity of azoalkanes through CB[7]-metal ion interactions in a two-phase system. ${ }^{359}$ 
Masson and Lu reported a multi-step catalytic cycle for the desilylation of trimethylsilylalkynl containing compounds via a heteroternary complex formed with $\mathrm{Ag}^{+}$ions and $\mathrm{CB}[7] .{ }^{342}$ The desilylation of (E35) in the presence of $\mathrm{CB}[7]$ and $\mathrm{Ag}^{+}$, which led to the formation of an alkyl-silver organometallic complex, was monitored through the ${ }^{1} \mathrm{H}$ NMR response of the trimethylsilanol byproduct (E37), complexed with the CB[7] cavity. The mechanism proposed for the reaction begins with (E35) forming a complex with CB[7] whereby the trimethylsilane group binds to the $\mathrm{CB}[7]$ cavity. A silver cation then forms a $\pi$-complex with the triple bond of (E35) and the carbonyl portal of the CB[7]. Molecule (E35) then undergoes a nucleophilic substitution with water, cleaving the trimethylsilyl group, yielding the trimethylsilanol by-product (E37) and a alkynyl-silver complex, which is subsequently hydrolyzed to form the desilyated alkyne (E36) and the regenerated silver cation.

Nau et al. extended the concept of metal-CB $[n]$ catalysis by utilizing such complexes to effect product formation from photoreactions involving azoalkanes in a two phase system. ${ }^{359}$ Firstly, the formation of complexes between $\mathrm{CB}$ [7] and metal ions, including $\mathrm{Fe}^{3+}, \mathrm{Co}^{2+}, \mathrm{Cu}^{2+}, \mathrm{Zn}^{2+}, \mathrm{Ag}^{+}$and $\mathrm{Ti}^{+}$, was confirmed by noting hypsochromic shifts and fluorescence intensity changes in optical titrations. The complexes were then tested for their activity towards the photoreactivity of 2,3-diazabicylo[2.2.2]oct-2-ene (E38) and diazabicylo[2.2.1]hept-2-ene (E41) in a two solvent system, consisting of water and pentane. Analysis of the organic reaction mixture from a reaction of (E38) by gas chromatography showed that product ratio of 65:35 for $(\mathbf{E} 39) /(\mathbf{E 4 0})$ in both the presence and absence of $\mathrm{CB}[7]$, and a ratio of 70:30 with just metal ions present. This ratio shifted to 87:13 in the presence of $\mathrm{CB}[7]$ and metal ions $\left(\mathrm{Ti}^{+}, \mathrm{Fe}^{3+}, \mathrm{Co}^{2+}, \mathrm{Ni}^{2+}, \mathrm{Cu}^{2+}\right.$ and $\left.\mathrm{Ag}^{+}\right)$. The preferential formation of the diene (E40) was theorized to have arisen from a triplet excited state caused by heavy-atom-doped intersystem crossing. The photoreaction of (E41) resulted in a $100 \%$ preferential conversion to housane (E43), over the secondary product cyclopentene (E42), in the presence and absence of $\mathrm{CB}[7]$, metal ions, and most metal-CB[7] 
complexes. A distinct change in reactivity occurred in the presence of the $\mathrm{CB}[7]-\mathrm{Ag}^{+} \mathrm{com}-$ plex, where a product ratio of 59:41 for (E43)/(E42) was observed. It was postulated that a photo-induced electron transfer was likely due to the new mechanism that led to the formation of cyclopentene, a mechanism that must be supported by the $\mathrm{CB}[7]-\mathrm{Ag}^{+}$complex, as no cyclopentene formed in the presence of $\mathrm{Ag}^{+}$alone, even at concentrations as high as $1 \mathrm{M}$. This work demonstrated the potential of $\mathrm{CB}[n]$-cation complexes in promoting otherwise unobtainable reaction products via photochemistry.

\subsection{Reaction inhibition by cucurbit[n]urils}

Cucurbiturils show a strong capacity to encapsulate guests within their hydrophobic cavities. Whilst we have seen that this can lead to the catalysis of reactions through efficient orientation of guests and carbonyl portal interactions, in addition to effecting reaction products through templating, the encapsulation of guests can also lead to the inhibition of reactions. In this way, $\mathrm{CB}[n] \mathrm{s}$ can act in much the same manner as a protecting group in organic synthesis, shielding specific molecules and functional moieties from chemical attack.

The redox chemistry of thiol groups and disulphide bridges have been manipulated through $\mathrm{CB}[6]$ binding. ${ }^{344}$ Kaifer et al. first highlighted that compounds such as cysteamine (E44) and cystamine (E45), and derivatives based on these compounds, can be encapsulated within $\mathrm{CB}[6]$ in an orientation such that the sulphur containing groups remain inside the hydrophobic cavity. They then investigated the oxidation and reduction of these compounds in the presence and absence of $\mathrm{CB}[6]$. When reacted with an excess of dithiothreitol (DTT), cystamine was shown to be reduce to the thiolated form, cysteamine, with the reaction coming to completion after approximately $1 \mathrm{~h}$. In the presence of $\mathrm{CB}[6]$, no formation of cysteamine was observed after $50 \mathrm{~h}$ of reaction time. Similar results were obtained for the oxidation of cysteamine, with no formation of cystamine in the presence of $\mathrm{CB}[6]$, whilst in its absence, $\mathrm{FeCl}_{3}$ was able to oxidize cystamine to cys- 
teamine to completion after approximately $6-7 \mathrm{~h}$. This reduction in reactivity was also seen when different oxidants were used, including dissolved oxygen and chloropicrin. This work highlighted the impressive ability of CBs to not only protect a species from chemical attack, but to also kinetically hinder both forward and reverse reactions as a result of host-guest complex formation.

Masson et al. demonstrated the use of $\mathrm{CB}[7]$ in modulating the chemiluminescence of Lucigenin (E46). ${ }^{360}$ Lucigenin undergoes chemiluminescent degradation upon the addition of sodium peroxide, which leads to the formation of dioxetane (E47). Dioxetane then undergoes homolytic cleavage to produce singlet excited acridone (E48), which relaxes to the ground state (E49) via photon emission. $\mathrm{CB}[7]$ was shown to modulate this reaction by binding to various ' $R$ ' group substituents of Lucigenin that are present on either side of the biacridine unit. The binding decreased dioxetane formation and dimmed the chemiluminescent reaction. Chemiluminescence could be restored through the addition of a competitive $\mathrm{CB}[7]$ guest. In this way, $\mathrm{CB}[7]$ can be utilized in regulating the rate of chemiluminescent reactions.

Cong et al. reported the use of $\mathrm{CB}[6]$ and $\mathrm{CB}[7]$ in the prevention of acylation of the anti-tuberculosis drug, isoniazid. ${ }^{362}$ For all acylating agents tested, stoichiometric additions of either $\mathrm{CB}[6]$ or $\mathrm{CB}[7]$ rendered isoniazid completely unreactive to acylation, with the initial rate of acylation decreasing between 10-100 fold in the presence of 0.8:1 CB to isoniazid. The mechanism for the protection was concluded to arise because of an increase in the $\mathrm{pK}_{a}$ value of the isoniazid, as a result of the amino protons binding to the carbonyl portals of $\mathrm{CB}$. This work demonstrates the potential for $\mathrm{CB}[n] \mathrm{s}$ to be used as drug carriers for isoniazid, increasing its bio-availability in the presence of acetylators.

$\mathrm{CB}[7]$ has also been shown to stabilize biologically active molecules from various chemical degradation pathways. Biczok et al. utilized inclusion complexes with CB[7] and sanguinarine, a natural biologically active benzophenanthridine alkaloid, to stave off photo-oxidation and nucleophilic attack. ${ }^{361}$ The iminium form (E50) of sanguinarine 


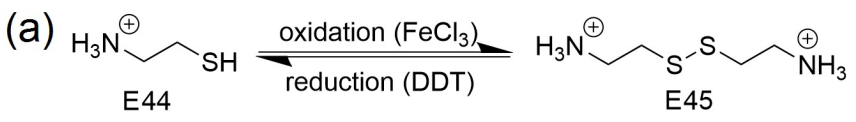

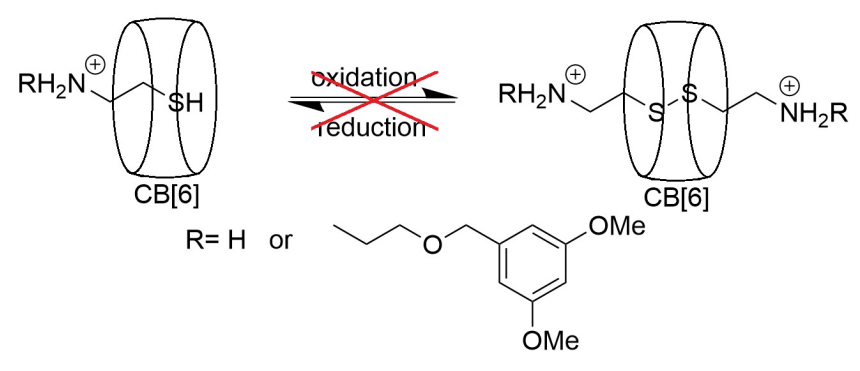

(b) $\mathrm{R} \quad \mathrm{R}$<smiles>[Z10]c1cccc2c1N([R])C1C=CC=CC1C2=O</smiles>

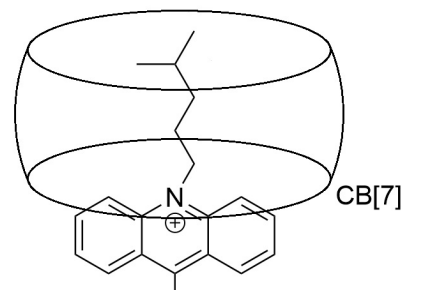

$R=M e$, 答

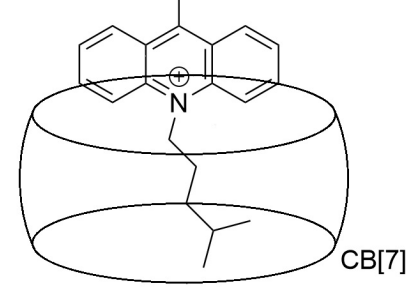

2:1 CB[7]-lucigenin

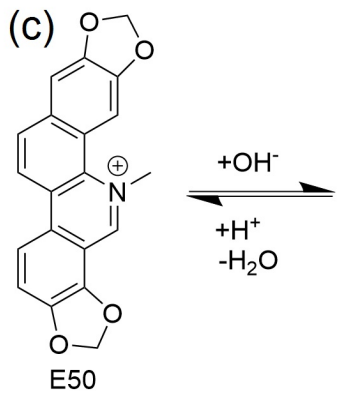

iminium form
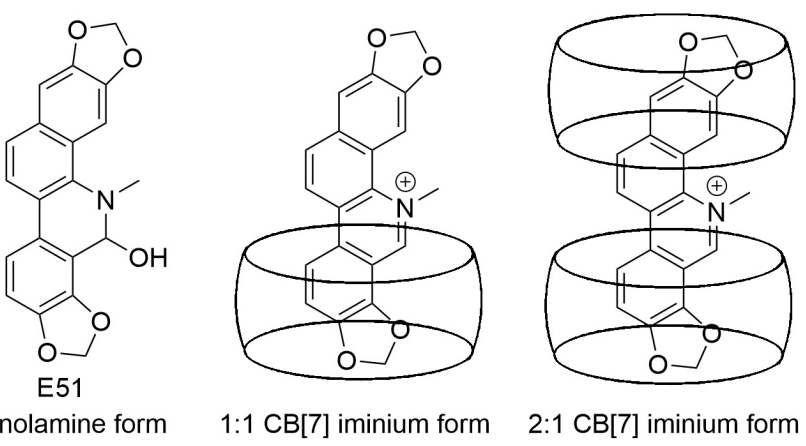

Figure 43: (a) Inhibition of redox reactions involving thiols and disulphides through protection within $\mathrm{CB}[6]$ cavities.et al. ${ }^{344}$ (b) The chemiluminescent reaction of lucigenin, modulated by $\mathrm{CB}[7] .{ }^{360}$ (c) Inhibition of the photo-oxidation and nucleophilic attack of sanguinarine by $\mathrm{CB}[7]$ encapsulation. ${ }^{361}$ 
formed both a 1:1, and 2:1 host-guest complexes with CB[7], with binding constants of $10^{6}$ $\mathrm{M}^{-1}$ and $10^{3} \mathrm{M}^{-1}$, respectively. The iminium form of sanguinarine is susceptible to nucleophilic attack by hydroxide ions at the 6 position, leading to the uncharged alkanolamine form (E51); a reaction that was prevented when complexed with CB[7]. The hydroxylated form of sanguinarine can also form a complex with $\mathrm{CB}$ [7], with a binding constant of $2.45 \times 10^{2} \mathrm{M}^{-1}$. The alkanolamine form can undergo an irreversible photo-oxidation reaction to produce 6-oxysanguinarine. This reaction was hindered by the presence of $\mathrm{CB}[7]$, likely through preventing oxygen from reaching the sanguinarine whilst encapsulated within the CB cavity.

In addition to modulating the rate of chemical reactions, $\mathrm{CB}[7]$ has been exploited to regulate the activity of enzymes. Ghosh et al. designed bi-functional inhibitor molecules with affinities for both $\mathrm{CB}$ [7] and either bovine carbonic anhydrase (BCA) or acetylcholinesterase (AChE). ${ }^{363}$ The addition of inhibitor molecules in stoichiometric ratios to BCA saw a decrease in its catalytic activity as a result of the blocking of its active site. The addition of $\mathrm{CB}[7]$ to the enzyme - inhibitor complex saw the formation of enzyme . inhibitor $\cdot C B[7]$ complexes that underwent prompt dissociation, releasing inhibitor $\cdot C B[7]$ and reforming the active enzyme. The inhibition could then be cycled by adding a competitive guest for $\mathrm{CB}[7]$, releasing the inhibitor molecule. Whilst $\mathrm{BCA}$ was shown to follow the inhibition cycle mentioned, AChE did not given the formation of stable complexes with both inhibition molecules and CB[7] simultaneously. This meant that CB[7] was unable to actively dissociate the inhibitor molecule from AChE. By using tailored inhibitor molecules with affinities for both enzymes and $\mathrm{CB}[7]$ macrocycles, cucurbiturils could play an important role in allowing for dynamic manipulation over the activity of enzymes. 


\section{Recognition by $\mathrm{CB}[n]$ in biological systems}

Molecular recognition and detection of biomolecules in aqueous solution, particularly peptides and proteins, is an important target within biomedical sectors, especially those focussed on sensing and separation. Research from the Brunsveld, Buschmann, Kim, Li, Nau, Scherman and Urbach groups has brought the art of amino acid complexation and recognition by $\mathrm{CB}[n]$ receptors forward to protein-protein conjugation and solution phase structure elucidation. This area has seen a large expansion in the number of publications on the binding of amino acids, peptides and proteins in the past 10 years and a wealth of knowledge of binding constants and conformations has been developed through utilization of a variety of spectroscopic and modeling techniques.

\subsection{Amino acid and short peptide recognition by $\mathrm{CB}[n]$}

An early example of short polypeptide recognition by $\mathrm{CB}[n]$ was reported by Buschmann et al. in 2005. ${ }^{364}$ This research determined binding constants for a small library of dior tripeptides through ion-dipole interactions between the protonated $\mathrm{N}$-terminus of the peptides and the portals of $\mathrm{CB}[6]$. The authors found that there was no significant dependence on peptide size to $\mathrm{CB}[6]$ binding, inferring that only an exclusion complex is formed (unlike alkylamino cations), likely resulting from the polarity of the peptide bonds. Nevertheless, the portal interaction is entropically favorable as two molecules of water are released from $\mathrm{CB}[6]$ upon peptide complexation.

Concurrently, Urbach and coworkers essentially pioneered the art of amino acid sensing when they reported the ability of $\mathrm{CB}[8] \cdot \mathrm{MV}$ to recognize and complex with tripeptides of glycine and a single tryptophan residue (WGG, GWG, GGW) forming charge-transfer complexes. This was monitored by UV absorption spectroscopy, fluorescence and NMR spectroscopies as well as ITC. ${ }^{130}$ The authors found WGG had the overall highest $K_{a}$

of the three peptides $\left(1.3 \times 10^{5} \mathrm{M}^{-1}\right.$, 6-fold higher than GWG and 40-fold higher than 
GGW) to $\mathrm{CB}[8] \cdot \mathrm{MV}$ and that electrostatic interactions of the C-terminus played a large part in the binding affinity on account of repulsion between the carbonyl portals of $\mathrm{CB}[8]$ and the carboxylate of the peptides. Binding of the tryptophan residue to the $\mathrm{CB}[8]$ cavity was accompanied by charge transfer complexation with MV and consequent fluorescence quenching of the indole group, as well as broadening of signals in ${ }^{1} \mathrm{H}$ NMR. This work was later extended into recognition of multivalent peptides by self-assembled receptors. ${ }^{365}$ Here peptide scaffolds were functionalized with multiple MV units (1-3) that each recruit one $\mathrm{CB}[8]$ host. This self-assembled receptor was then able to bind in a 1:1 fashion with identical peptides bearing complementary tryptophan residues in place of the MV of the receptors, Figure 44a.

a)
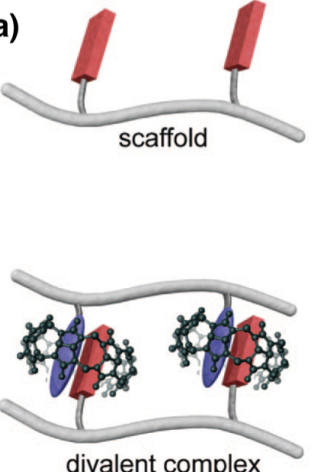
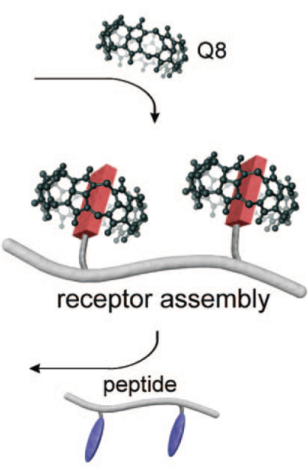

b)

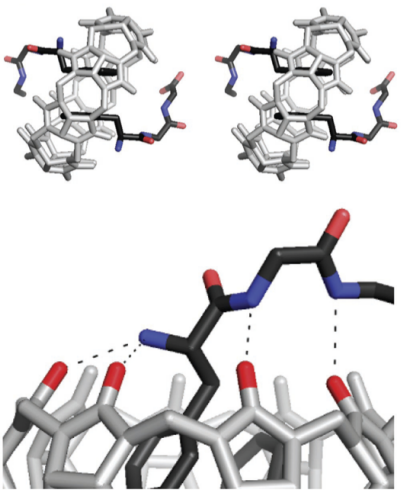

c)

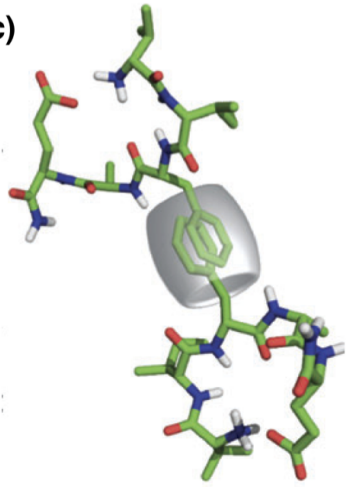

Figure 44: a) Self-assembled multivalent receptors of "MV-peptide" and CB[8] complex aromatic residues in complementary peptides, figure reproduced in part with permissions from Reczek et al. J. Am. Chem. Soc. 2009, 131, 2408-2415, Copyright 2009 American Chemical Society. b) Crystal structure of two FGG peptides complexed to CB[8]. Hydrogen bonding interactions between peptide backbone and $\mathrm{CB}[8]$ carbonyl portals exemplified by dashed lines, figure reproduced in part with permissions from Heitmann et al. J. Am. Chem. Soc. 2006, 128, 12574-12581, Copyright 2006 American Chemical Society. c) 2:1 complexation of pentapeptides to CB[8] via mid-chain Phe residues, figure reproduced in part from Sonzini et al. Chem. Commun. 2013, 49, 8779-8781 with permission from The Royal Society of Chemistry

Urbach et al. also observed 2:1 binding of aromatic amino acids to $\mathrm{CB}[8] \cdot{ }^{165}$ Consistent with the previous study, tripeptides consisting of 2 glycine residues and a single tryptophan or phenylalanine were investigated and it was again found that the distance of the aromatic residue from the C-terminus had a large effect on binding affinity. Sequential 
binding modelling of ITC data demonstrated, in the case of WGG, the second binding constant is a magnitude lower than the first, however, this could not be determined for FGG due to cooperativity in binding. WGG was found to bind to $\mathrm{CB}[8]$ in a 2:1 fashion in a non-cooperative manner unlike FGG whereby ITC data suggests positive cooperativity. The authors also managed to isolate a crystal structure of FGG bound in a 2:1 fashion to $\mathrm{CB}[8]$, with the aromatic residues encapsulated in a stacking geometry within the cavity, Figure 44b. Hydrogen bonding interactions between the peptide N-H's and $\mathrm{CB}[8]$ carbonyl portals were also apparent. Other aromatic amino acid residues were investigated (tyrosine, histidine), however, binding to $\mathrm{CB}[8]$ was not observed for these derivatives.

The study of 2:1 binding of middle-chain phenylalanine residues in pentapeptides to $\mathrm{CB}[8]$ has also been investigated by Scherman et al. ${ }^{164}$ This is the first example of strong 2:1 binding in a longer peptide chain where the Phe residue is not located at the $\mathrm{N}$-terminus. The pentapeptides selected were based on the aggregation promoting region of wild type human amyloid $\beta(\mathrm{A} \beta)$, a peptide that has been linked to the development of Alzheimer's disease. The A $\beta$ fragments explored were AEFRH, LVFIA, VIFAE, of which the latter two were overlapping regions of the $\mathrm{A} \beta$ sequence but with one of the $F$ residues exchanged for I (isoleucine). In wild type $\mathrm{A} \beta$ this region actually contains two neighboring Phe units and the authors chose to replace one of these residues in each sequence with isoleucine to ease synthesis and analysis of results when combined with $\mathrm{CB}$ [8]. 2:1 binding modes were confirmed by fluorescence and NMR spectroscopy and ITC analyses and despite differing charges, hydrophobicity and steric hindrance, all sequences formed strong homoternary complexes on the order of $10^{9}-10^{13} \mathrm{M}^{-2}$, Figure $44 \mathrm{c}$. This study demonstrates $\mathrm{CB}[8]$ is able to access potentially hindered Phe residues in non-Nterminal sites of peptides and may also hold advantages to the future of understanding $\mathrm{A} \beta$ fibrillation mechanisms in Alzheimer's disease.

1:1 binding of phenylalanine derivatives to $\mathrm{CB}[7]$ has also been investigated by Urbach and coworkers. Remarkably one derivative, 4-aminomethyl-phenylalanine, was re- 
ported to have a $\mathrm{K}_{d}$ of $0.95 \mathrm{nM}$ (500x stronger affinity than phenylalanine). ${ }^{366}$ However, biomolecule detection often has to be performed at much lower concentrations. Other chemical modifications of phenylalanine were investigated such as $t$-butyl and hydroxyl groups at the 4-phenyl position, but none of these modifications provided such a stark increase in binding affinity as the amino-methyl group. A later report by Urbach et al. detailed nanomolar detection of peptides harnessing $\mathrm{CB}[8]$ complexes of tetramethylbenzobisimidazolium (MBBI). ${ }^{97} \mathrm{MBBI} \cdot \mathrm{CB}[8]$ was shown to have an intriguing response to tripeptides Tyr-Leu-Ala and Tyr-Ala-Leu within nano-micromolar detection limits. In the former case, a fluorescence enhancement was observed upon combination, elucidating displacement of MBBI from $\mathrm{CB}[8]$, and this result was confirmed by NMR studies. In the latter example, fluorescence was seemingly quenched by the formation of a 1:1:1 chargetransfer inclusion complex of $\mathrm{CB}[8]$, Tyr-Ala-Leu and MBBI. Therefore, MBBI.CB[8] is able to act as both a nanomolar "fluorescence-off" receptor for Tyr-Ala-Leu and "fluorescence on" receptor of Tyr-Leu-Ala, simultaneously. This is the first example of highly specific recognition of $\mathrm{N}$-terminal Tyr tripeptides by $\mathrm{CB}[8]$, whereby in certain cases, the peptide chain can fold and be mostly included within the $\mathrm{CB}[8]$ cavity (as modelled) at the expense of dissociation of MBBI. The authors suggest that given the minimal structure of this Tyr-Leu-Ala peptide, it could easily be engineered into recombinant proteins as an affinity tag.

Urbach et al. also demonstrated inhibition of a nonspecific exopeptidase, porcine amino peptidase $\mathrm{N}(\mathrm{APN})$ with $\mathrm{CB}[7]$ via binding of phenylalanine units present within the peptide. ${ }^{367} \mathrm{~N}$-exopeptidases degrade peptides by sequential cleavage of amino acids from the N-terminus. With CB[7] bound to a Phe residue within a peptide sequence (ThrGly-Ala-Phe-Met), cleavage of the amide bond was completely inhibited upon reaching the CB7.Phe complex and therefore Phe-Met was stable to APN degradation. The authors elude to the applicability of this system in the protection of longer peptides from APNs, which could prolong peptide lifetimes in vitro and in vivo. 
Collaboration between the Urbach and Nau groups led to the development of a tandem assay whereby discrimination of $\mathrm{CB}[7]$ affinity for fluorescent dyes and peptides allowed for determination of protease substrate selectivity and inhibitor activities. ${ }^{368}$ In this study, mixtures of acridine orange (fluorescent dye), CB[7], peptides (with Phe residues) and proteases were formulated. Initially acridine orange is encapsulated by $\mathrm{CB}[7]$ on account of a stronger binding constant than the mid-chain Phe residue of the peptide and fluoresces. Upon cleavage of the peptide by proteases, the Phe unit was revealed in the new N-terminus position, the acridine orange was displaced from the $\mathrm{CB}[7]$ by the peptide and fluorescence of the dye quenched. This system was utilized to quantify the activity of several proteases but also able to determine the activity of protease inhibitors. This method proved to be a robust way to determine both enzyme and inhibitor activity at nano-molar levels in a label free manner. However, it is limited to peptides that yield products with $\mathrm{N}$-terminal aromatic amino acids upon enzymatic degradation.

This area has seen excellent progression over the past decade from recognition of amino acids by $\mathrm{CB}[8] \cdot \mathrm{MV}$ towards the development of sensitive detection assays and dimerization of larger peptides by $\mathrm{CB}[8]$.

\subsection{Non-covalent protein conjugation}

In more recent literature, the groups of Scherman, Brunsveld and various others have developed methods to conjugate macromolecules to larger proteins via $\mathrm{CB}[8]$ recognition. An early example of non-covalent conjugation was of PEG to BSA through CB[8] ternary complex formation, as reported by Scherman et al. in 2011, Figure 45a. ${ }^{143}$ PEGylated proteins have distinct physicochemical properties (resistance to biodegradation, etc.) and therefore bioconjugation of polymers in a site-specific and dynamic manner is applicable to many biological sectors, especially therapeutic protein delivery. In this example, a BSA fraction was functionalized with a single naphthoxy moiety at its only free thiol cystine residue. Combining this in solution with PEG-MV and $\mathrm{CB}[8]$ led to ternary complex 
formation and a PEG-BSA bioconjugate was observed by DOSY NMR, DLS, UV/vis and fluorescence spectroscopies and ITC. This work, as described in the authors conclusions, demonstrated controlled supramolecular formation of PEG-protein bioconjugates, which is a proof of concept for further translation or potentially protein-protein supramolecular conjugation.

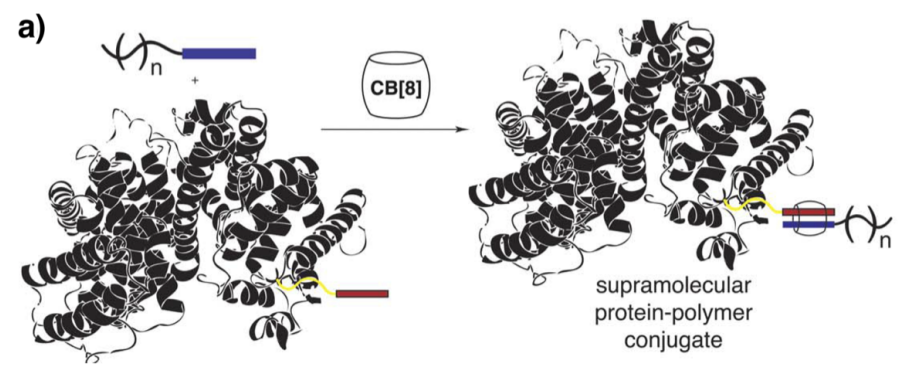

c)
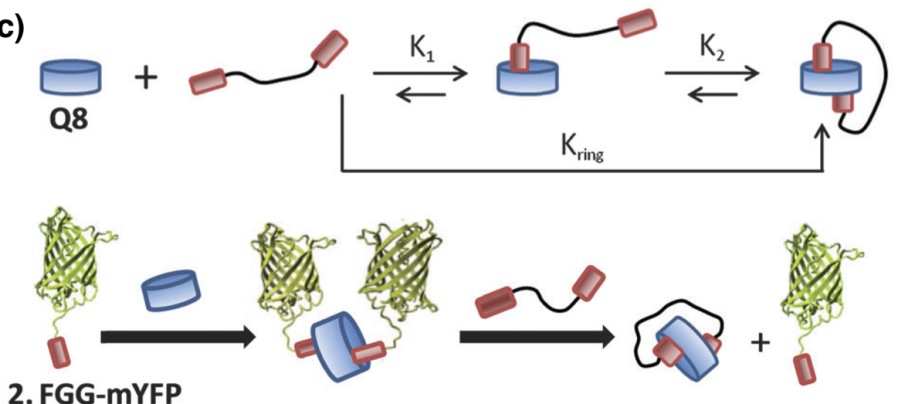

b)
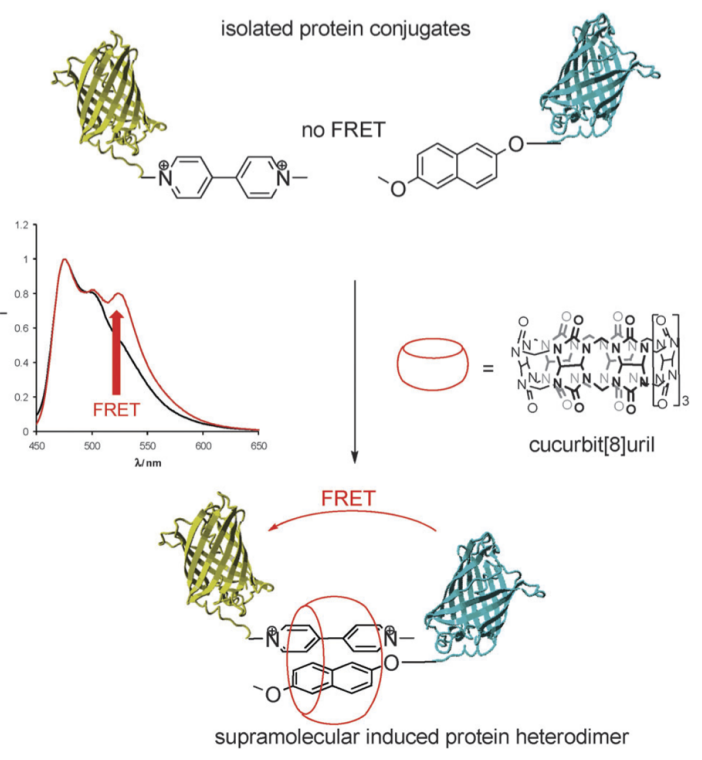

Figure 45: a) Reversible BSA PEGylation through hetero-ternay complex formation with CB[8], figure reproduced in part from Biedermann et al. Chem. Sci. 2011, 2, 279-286. b) Dimerization of protein FRET pairs with $\mathrm{CB}$ [8], figure reproduced from Uhlenheuer et al. Chem. Commun, 2011, 47, 6798-6800 c) Controlling protein dimerization by utilizing FGG-PEG-GGF polymer, figure reproduced in part from Ramaekers et al. Chem. Commun. 2015, 51, 3147-3150. All images reproduced with permissions from The Royal Society of Chemistry.

Protein-protein heterodimerization of MV and Np bearing proteins with $\mathrm{CB}[8]$ was reported by Brunsveld et al. several months later. ${ }^{144}$ This study demonstrated the selective dimerization of two different proteins, one bearing MV to a second protein bearing $\mathrm{Np}$, following on from previous work by Brunsveld, which was limited to dimerization of identical proteins through 2:1 complexation of FGG-protein sequences with CB[8], Figure $45 \mathrm{~b} .{ }^{282}$ In both cases protein FRET pairs could be dimerized and therefore the assembly process studied by fluorescence spectroscopy. A follow-up SAXS study eluded to the 
compact nature of packing of such dimers when complexed in solution. ${ }^{369}$ Collaboration between the Brunsveld and Dankers groups saw the production of (FGGG) ${ }_{2}$ penta(ethylene glycol), which could form ring structures on the binding of its 2 FGGG units to $\mathrm{CB}[8]$ and modulate protein dimerization with more control than simple competitive FGG molecules, Figure $45 c .{ }^{281} \mathrm{CB}[8]$ mediated homotetramerization of fluorescent proteins was later reported by Brunsveld and coworkers. ${ }^{370}$ This was achieved by combining the 2:1 complexation motif of FGG (encoded at the N-terminus of many proteins) to $\mathrm{CB}[8]$ with intrinsic affinities between proteins, essentially producing supramolecular dimers of dimers.

Brunsveld and coworkers later reported a further study on the dimerization of inactive enzyme monomers with $\mathrm{CB}[8]$ to form an active enzyme supramolecular dimer. ${ }^{371}$ Here Caspase-9, which is only active in its dimeric form, was complexed together via recognition of FGG moieties by CB[8]. Perhaps most importantly, the activity of the enzyme was not compromized by the supramolecular elements that induce dimerization and a great deal of molecular control over the system was achieved, i.e. $\mathrm{CB}[8]$ concentration controlled the degree of dimerization and therefore activity of the Caspase solution.

In summary, significant progress has been made in the understanding of protein conjugation facilitated by $\mathrm{CB}[8]$ recognition motifs and this insight is starting to be translated towards modulation in protein activity showing great promise for the future applicability of this field.

\subsection{Further application to biochemistry}

Urbach and coworkers reported a 1:1 binding stoichiometry between $\mathrm{CB}[7]$ and insulin, at the N-terminal phenylalanine of the insulin B chain. ${ }^{96}$ This is a particularly interesting result as there are three Phe residues in the insulin sequence, however, replacement experiments of these other residues confirmed that it is solely the N-terminal Phe that binds to CB[7]. This is starkly different to other methods of recognizing proteins as no modifications to the peptide sequence in terms of histidine tagging have been performed. 
Recognition has simply been achieved through mixing the native protein with $\mathrm{CB}[7]$ and large binding constants upwards of $10^{6} \mathrm{M}^{-1}$ were reported.

Kim and coworkers showed that $\mathrm{CB}[7]$ binding to Phe residues of insulin and amyloid $\beta$ prevented aggregation of such proteins. ${ }^{372}$ This is very important for both insulin storage and understanding development of Alzheimer's disease through amyloid aggregation. These studies show that by masking the hydrophobic residues of $A \beta$ or insulin with $\mathrm{CB}[7]$, or at least increasing steric hindrance around these regions via complex formation, significant inhibition of the aggregation process can be achieved. Through a variety of biological assays and binding studies the authors demonstrate that $\mathrm{CB}[7]-\mathrm{Phe}$ complexation is much faster than protein-protein dimerization and the Phe-specific binding of $\mathrm{CB}[7]$ successfully modulates intermolecular interactions, preventing fibrillation processes, and is therefore a pathway for new potential treatments for amyloid linked diseases.

A 2011 report by Kim et al. demonstrated the gas phase host guest chemistry of $\mathrm{CB}[6]$ and Lys containing peptides. ${ }^{373}$ Through collisional activation, $\mathrm{CB}[6]$ was found to efficiently complex to Lys residues of model peptides in the gas phase, often resulting in common fragmentation products. This was used to elucidate protein structure, specifically in the case of ubiquitin, by employing tandem mass spectrometry techniques on $\mathrm{CB}[6]-$ protein complexes formed in aqueous conditions to determine solution phase conformations. A follow-up study investigated the conformational changes of ubiquitin proteins upon $\mathrm{CB}[6]$ complexation in the gas phase, at a variety of different charge states, of which specific Lys residues complexed with $\mathrm{CB}[6]$ were able to be determined. $\mathrm{CB}[6]$ complexes inhibited salt bridge formations and therefore triggered conformation changes. This could be reversed and the protein refolded upon CB[6] detachment. ${ }^{374}$ In the future this research will hopefully be translated to other protein structures and improve mass spectrometry techniques for determining protein structure, as well as determining protein sequences that in turn determine higher order conformations. 
Inhibition of DNA restriction enzymes by $\mathrm{CB}[6]$ and $\mathrm{CB}[7]$ was reported by Pischel and collaborators. ${ }^{375}$ In this example binding of $\mathrm{CB}[6]$ and $\mathrm{CB}[7]$ to DNA restriction enzymes is hypothesized to be the cause of inhibition of DNA hydrolysis, however, the authors were unable to determine exactly which residues of the restriction enzyme were bound by the CBs. It was shown that DNA hydrolysis could be reactivated by simple addition of spermine, which competes for the macrocycle cavity, likely releasing the restriction enzymes and allowing continuation of DNA hydrolysis.

\subsection{Profiling toxicity of cucurbit $[n]$ uril}

2010 saw two reports showing $\mathrm{CB}[n]$ type containers exhibiting good biocompatibility profiles. Isaacs, Briken and coworkers examined the viability of HepG2 (liver derived) and RAW264.7 (macrophage) cells after $24 \mathrm{~h}$ exposure to CB[5] and CB[7]. ${ }^{100}$ Both cell lines showed good tolerance to $\mathrm{CB}[5]$ and $\mathrm{CB}[7]$ up to $1 \mathrm{mM}$ with cell viabilities exceeding $90 \%$. Moreover, the internalization of CBs with encapsulated FITC by macrophages was proven to be very efficient and treatment of mycobacteria with $\mathrm{CB}[7]$-bound drug molecules did not see any significant decrease in efficacy of the therapeutic compound. Furthermore, acyclic analogues of $\mathrm{CB}[n]$ were also studied and the cells also expressed high tolerance for such compounds beyond usual therapeutic levels.

Day, Nau and coworkers also explored the in vitro activity of $\mathrm{CB}[7]$ and $\mathrm{CB}[8]$, specifically against $\mathrm{CHO}-\mathrm{K} 1$ cells (ovarian cell line from hamsters). ${ }^{99} \mathrm{An} \mathrm{IC}_{50}$ value of $0.53 \pm 0.02 \mathrm{mM}$ was determined, which roughly corresponds to $620 \mathrm{mg} \mathrm{kg}^{-1}$ of cell material. The authors also tested $\mathrm{CB}[8]$ toxicity and found that the macrocycle expressed no significant toxicity within its solubility limits. Further to these results, live cell imaging of cells exposed to non-lethal $\mathrm{CB}[7]$ concentrations demonstrated that there were no detrimental or adverse effect on the cellular integrity.

Progressing towards in vivo studies on mice, intravenous injections determined a maximum tolerable dose of $\mathrm{CB}[7]$ to be $250 \mathrm{mg} \mathrm{kg}^{-1}$ with the mice initially losing some su- 
perficial weight but recovering within 5-8 days. For oral administration, a $600 \mathrm{mg} \mathrm{kg} \mathrm{kg}^{-1}$ one-off dose of 1:1 CB[7]:CB[8] mixture did not give rise to any significant toxicity.

The Wheate group studied the ex vivo toxicology of $\mathrm{CB}[6]$ and $\mathrm{CB}[7]$ under more specific circumstances, analyzing the neuro-, myo- and cardiotoxic activity of the compounds. ${ }^{101}$ To study neurotoxicity, a mouse sciatic nerve (removed from between the knee and spinal cord) was exposed to $1 \mathrm{mM}$ solutions of $\mathrm{CB}[6]$ or $\mathrm{CB}[7]$ and the conductivity of the nerve monitored for $2 \mathrm{~h}$, no significant neurotoxicity was observed. Furthermore, cisplatin.CB[7] did not show a decrease in neurotoxicity as compared to free cisplatin. Myotoxicity was also studied by extracting biventer cervicis nerve-muscles from chicks and their contraction response to chemical or electrical stimuli was monitored before and after exposure to $0.3 \mathrm{mM} \mathrm{CB}[6]$ and $\mathrm{CB}[7]$ solutions. $\mathrm{CB}[6]$ caused only a very small change in the strength of muscle contraction so can be considered relatively safe. However, $\mathrm{CB}[7]$ had a much greater effect on muscle contraction and the authors hypothesize that this is due to $\mathrm{CB}[7]$ binding to postsynaptic nicotinic receptors, interfering with the depolarization ability of the muscle membrane. $\beta$-cyclodextrin exposure also resulted in small decreases in muscle contraction and was not myotoxic in vivo and may possibly also be the case for $\mathrm{CB}[6]$ and $\mathrm{CB}[7]$. Cardiotoxicity was examined using a similar method but with rat heart atria. Both $\mathrm{CB}[6]$ and $\mathrm{CB}[7]$ showed significant ex vivo cardiotoxicity, exemplified by changes in contraction forces. However, the authors of this study conclude that these experiments represent very high exposure levels to the macrocycles with direct contact with the tissues and so may not be accurate predictions of activity in human clinical models.

To better understand specific organ toxicity in vivo, Wang and collaborators employed zebrafish models to further study the contribution of CB[7] exposure to neuro-, myoand cardiotoxicity as well as hepatotoxicity, developmental toxicity, locomotion and behavioral toxicity. ${ }^{102}$ Mortality studies determined an $\mathrm{LD}_{50}$ (lethal dose) to be at $750 \mu \mathrm{M}$ when zebrafish were incubated with CB[7] for 2 days. No morphological abnormalities 
were observed on developing zebrafish exposed to even the higher, more toxic, levels of $\mathrm{CB}[7]$. Some detrimental effects on cardioactivity were observed on zebrafish exposed to $\mathrm{CB}[7]$ concentrations greater than $500 \mu \mathrm{M}$ including elevated heart rates, thinning of the atrium, distorted heart shape, decreased stroke volume and decreased cardiac output, which is consistent with the ex vivo study by Wheate. In terms of locomotive effects, extended exposure to higher concentrations of CB[7] significantly shortened swimming distances of zebrafish, which could be a factor of the observed cardiotoxicity, however, the authors reasoned that this was more likely a neurotoxic effect, conflicting with the Wheate ex vivo study. The authors did not observe any hepatotoxicity on zebrafish embryos, demonstrating a high degree of tolerability of $\mathrm{CB}[7]$ by the liver. This study is promising as detrimental effects were not observed below usual therapeutic concentrations of most drug compounds, suggesting a reasonable level of tolerance of $\mathrm{CB}$ [7] in vivo and therefore warranting much further study in larger animal models.

\subsection{Outlook}

A considerable amount of work has been carried out in the area of peptide and protein supramolecular complexation with $\mathrm{CB}[n]$ and understanding of aromatic amino acid residues, cavity hydrophobic interactions, hydrogen bonding to portals and cooperative interactions in the example of $\mathrm{CB}[8]$ are progressing from the work of several groups. Protein dimerization is particularly exciting in terms of controlling activity and stability and many interesting studies into other forms of bio-conjugation harnessing such host-guest recognition are likely on the horizon. Particularly remarkable studies are those of insulin and amyloid $\beta$ and how the interactions of specific Phe residues modulates aggregation pathways. With these reported methodical studies in hand it is not difficult to imagine how many more $\mathrm{CB}[n]$-biomolecule interactions will come to light in the near future with new protocols to control and regulate protein activity. It is also promising that initial studies suggesting application of CB-based materials to human clinical settings will not 
be hampered by issues of toxicity.

\section{Summary and outlook}

In just over three decades since the isolation and full characterization of the first cucur$\operatorname{bit}[n]$ uril homologue, this macrocyclic family has transformed from a laboratory bench curiosity into a compound of industrial significance. The widespread number of fields that $\mathrm{CB}[n]$ has found applications in is remarkable. Ranging from applications in (but not limited to) materials design, development of next generation sensors, sophistication of medical techniques through efficient drug delivery and encapsulation, potential in energy harnessing through its catalytic properties and green technology, $\mathrm{CB}[n]$ has been an exceptional contestant in all these fields. $\mathrm{CB}[n]$ has also proven itself as a versatile tool in fundamental research such as in separation science, as a precise molecular ruler for plasmonics, and as a catalytic reagent and nanoreactor. In this review, we have intended to focus on themes related to $\mathrm{CB}[n]$ that have not been presented in great detail in the literature prior to its writing. For example, we discuss the gas binding properties of $\mathrm{CB}[n]$ and the interaction of $\mathrm{CB}[n]$ with surfaces and their interesting applications. We have also highlighted the recent progress in various fields that have utilized the chemical and physical properties of $\mathrm{CB}[n]$ in an innovative manner including polymeric materials and networks as well as biological systems.

The exploration of the complexation capabilities of $\mathrm{CB}[n]$ with various guest molecules have led to the discovery of a plethora of species that are of scientific interest. New guests are constantly being reported providing unique insights into the understanding of the molecular recognition properties of $\mathrm{CB}[n]$. The advent of sophisticated characterization techniques has complemented research efforts in elucidating the fundamental processes behind $\mathrm{CB}[n]$ host-guest chemistry. In addition, this review on $\mathrm{CB}[n]$ notably presents the first comprehensive tabulation of all the binding data that can be readily found in the lit- 
erature through the first half of 2015. The similarities as well as disparities in the binding constants that result from different techniques and measurement conditions are unmistakable. Therefore, the need to standardize measurement and characterization methods is critical underpinning the importance of reporting precise conditions in which the measurements are taken.

Driven by the shortcomings exhibited by several $\mathrm{CB}[n]$ homologues arising from their extremely low water solubility, various strategies to functionalize $\mathrm{CB}[n]$ have been pursued. Additionally, the functionalized host molecules have opened up possibilities to tether them to surfaces and polymeric materials. Although the topic has not been discussed in great detail in this review, the increasing pace of research activity and growing interest in controlled functionalization of CB derivatives is apparent and will likely warrant a dedicated review itself in the next few years. 


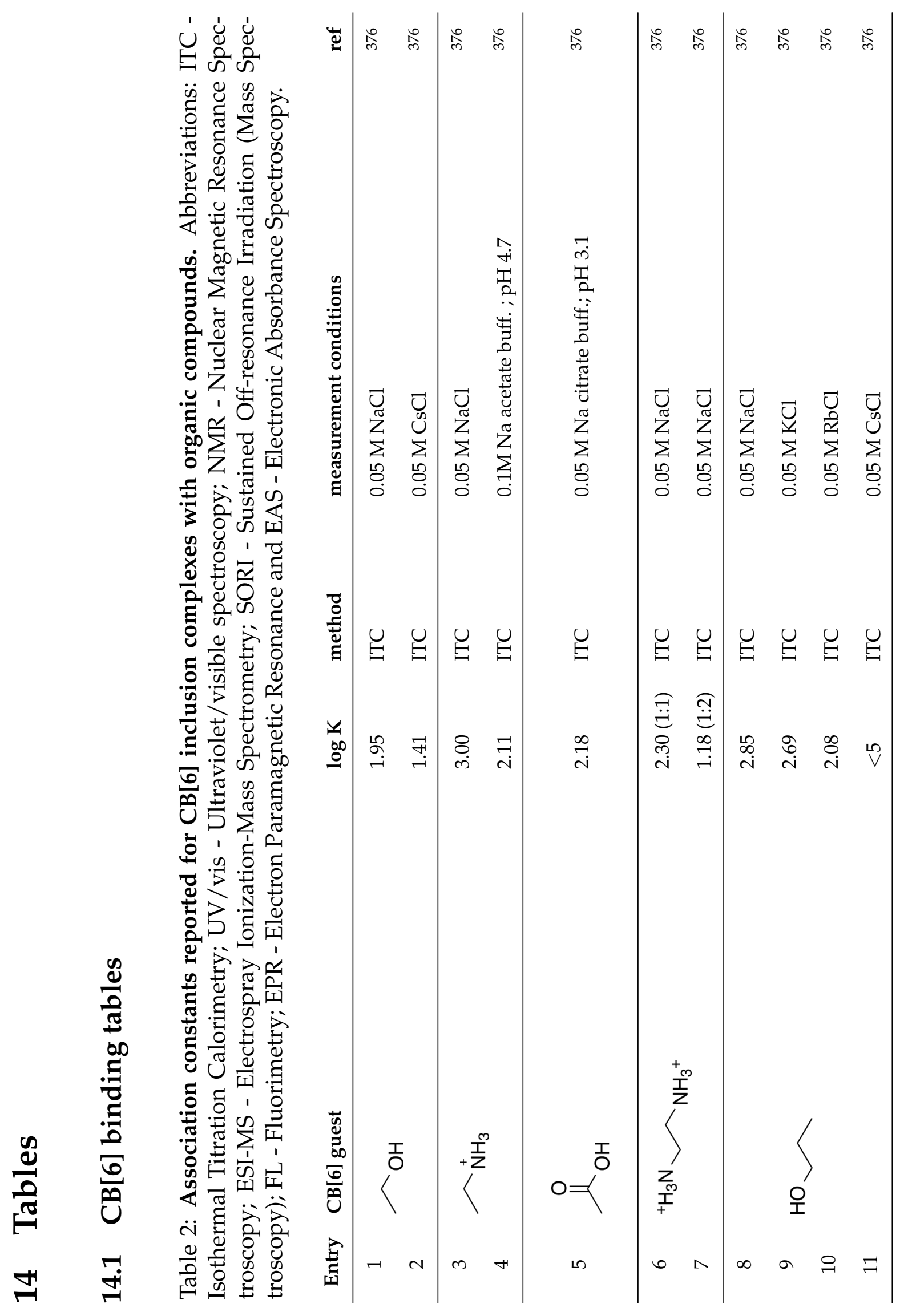




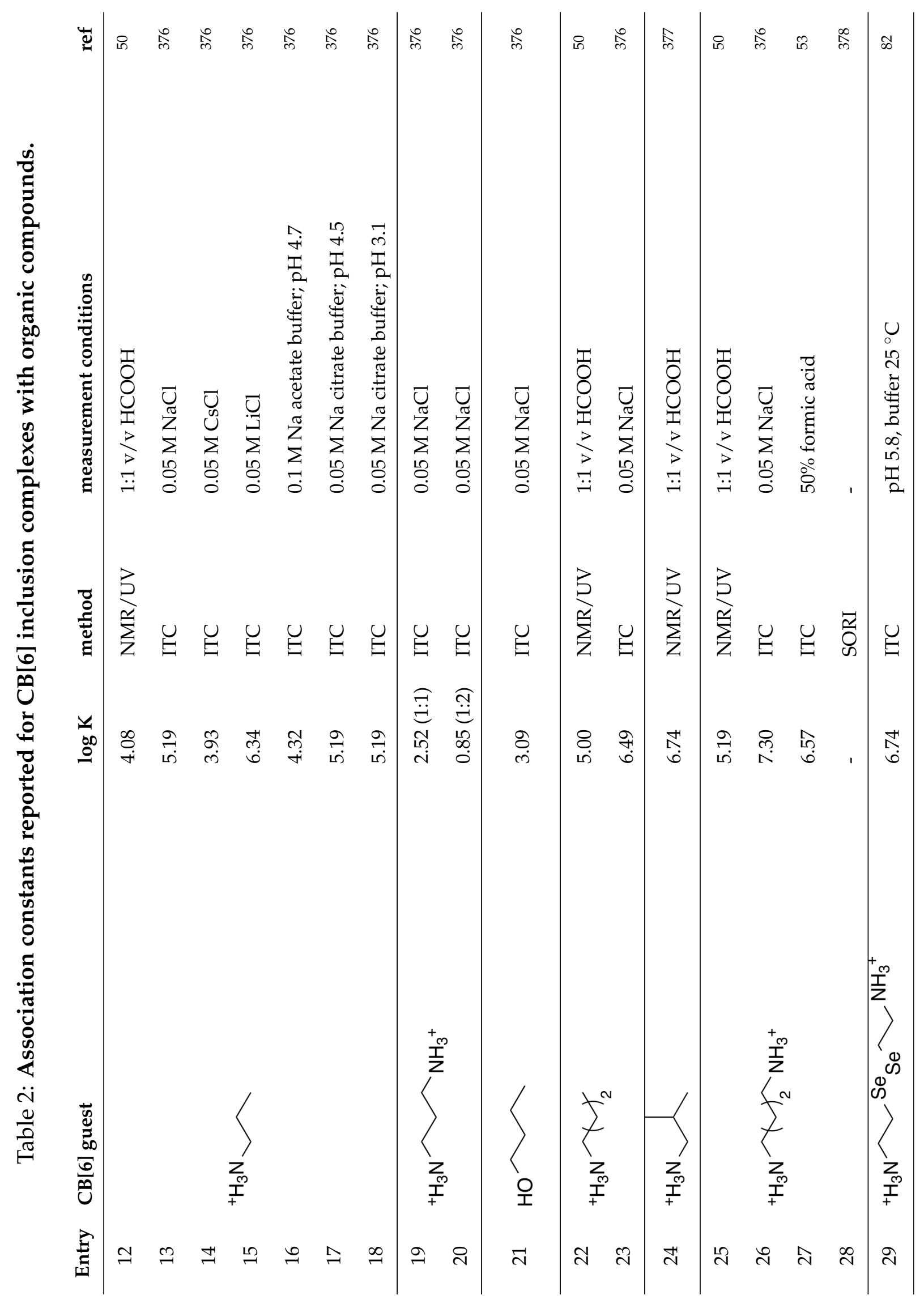




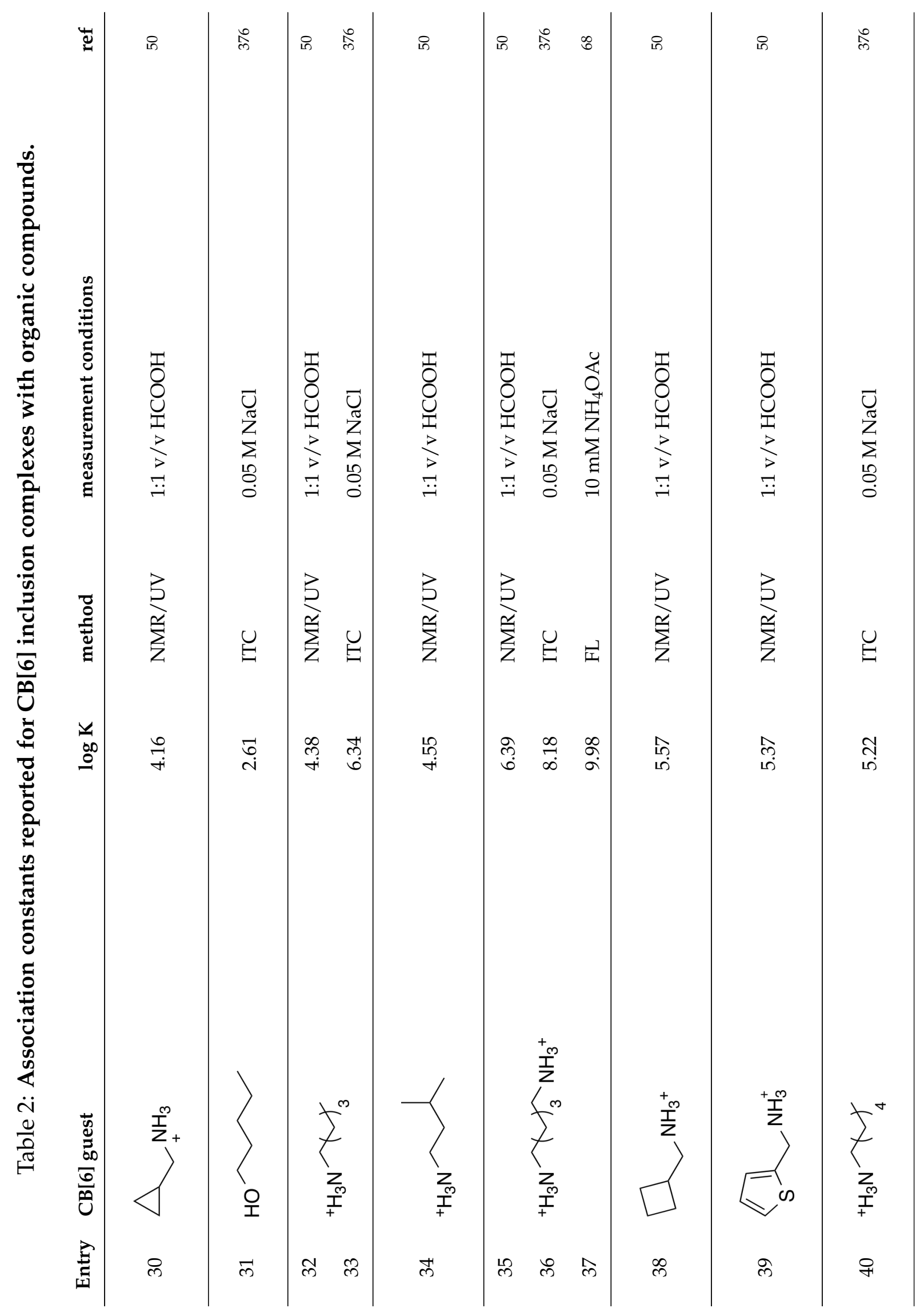




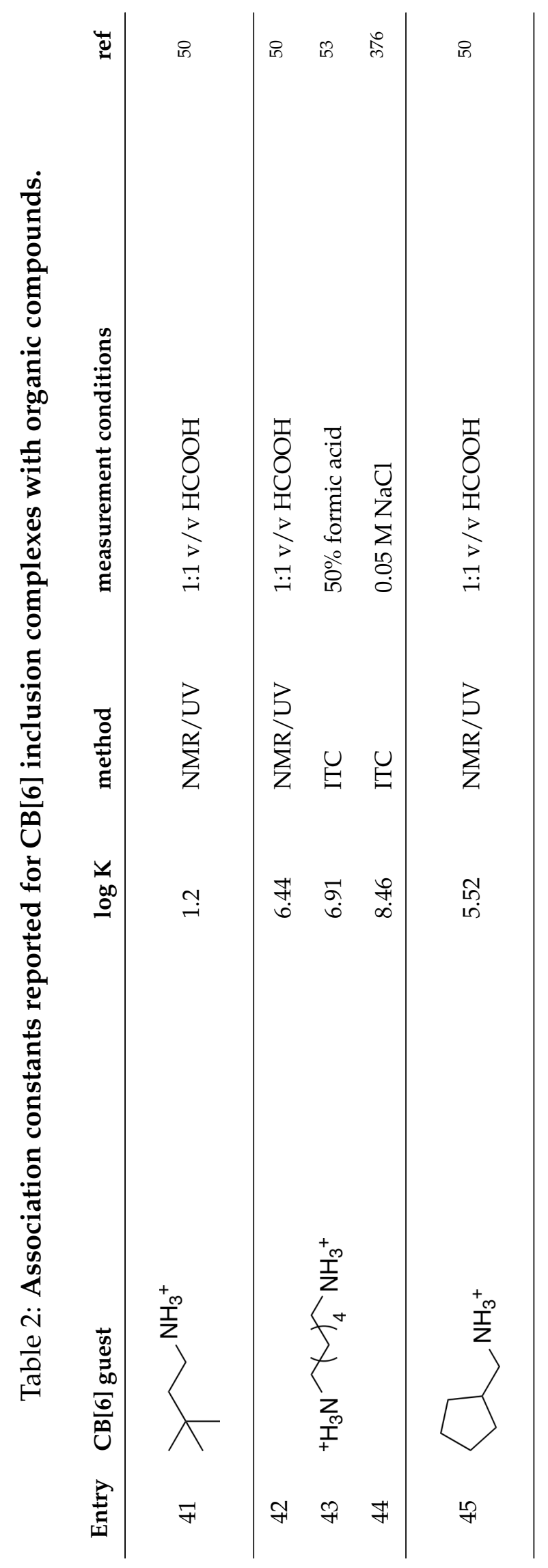




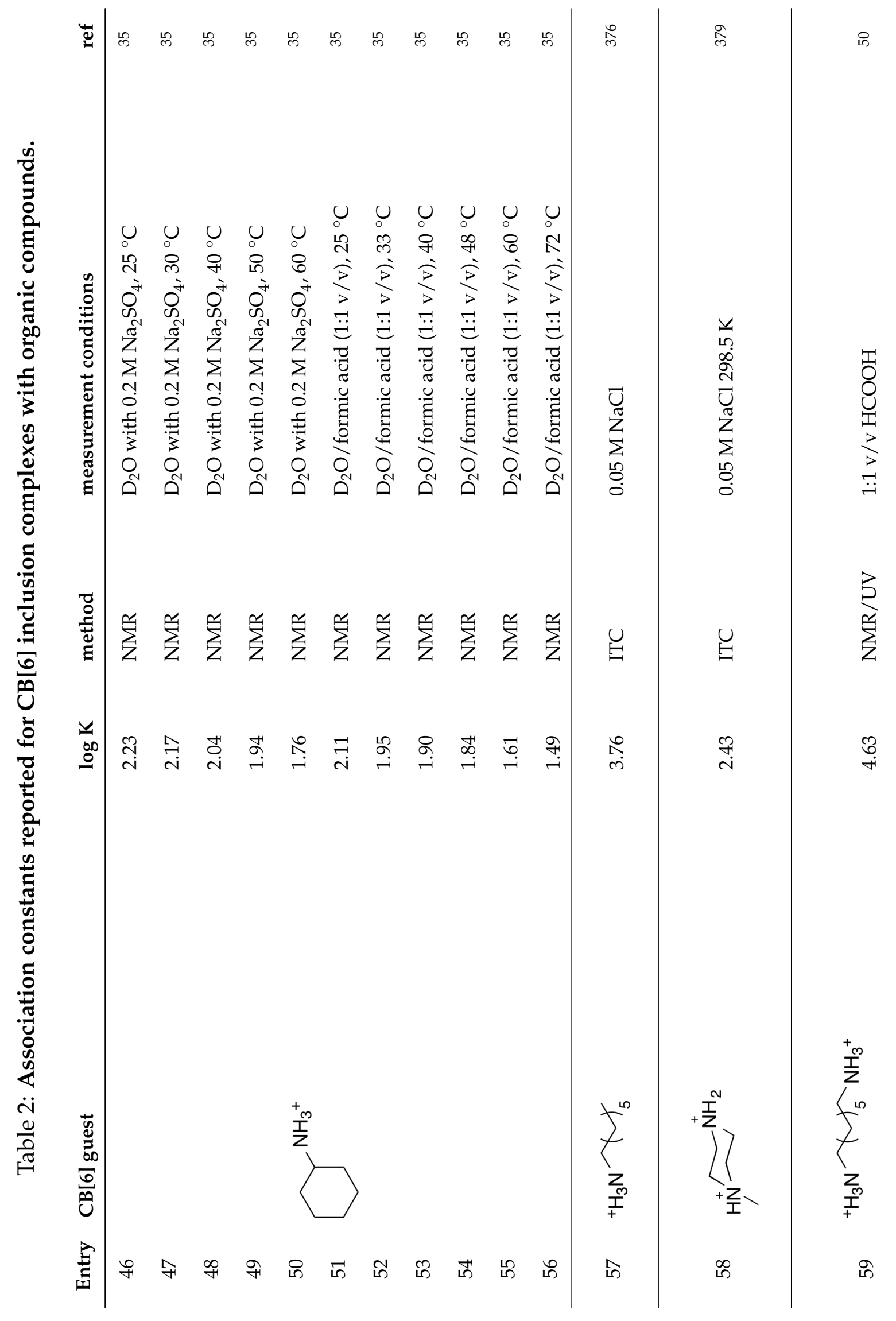




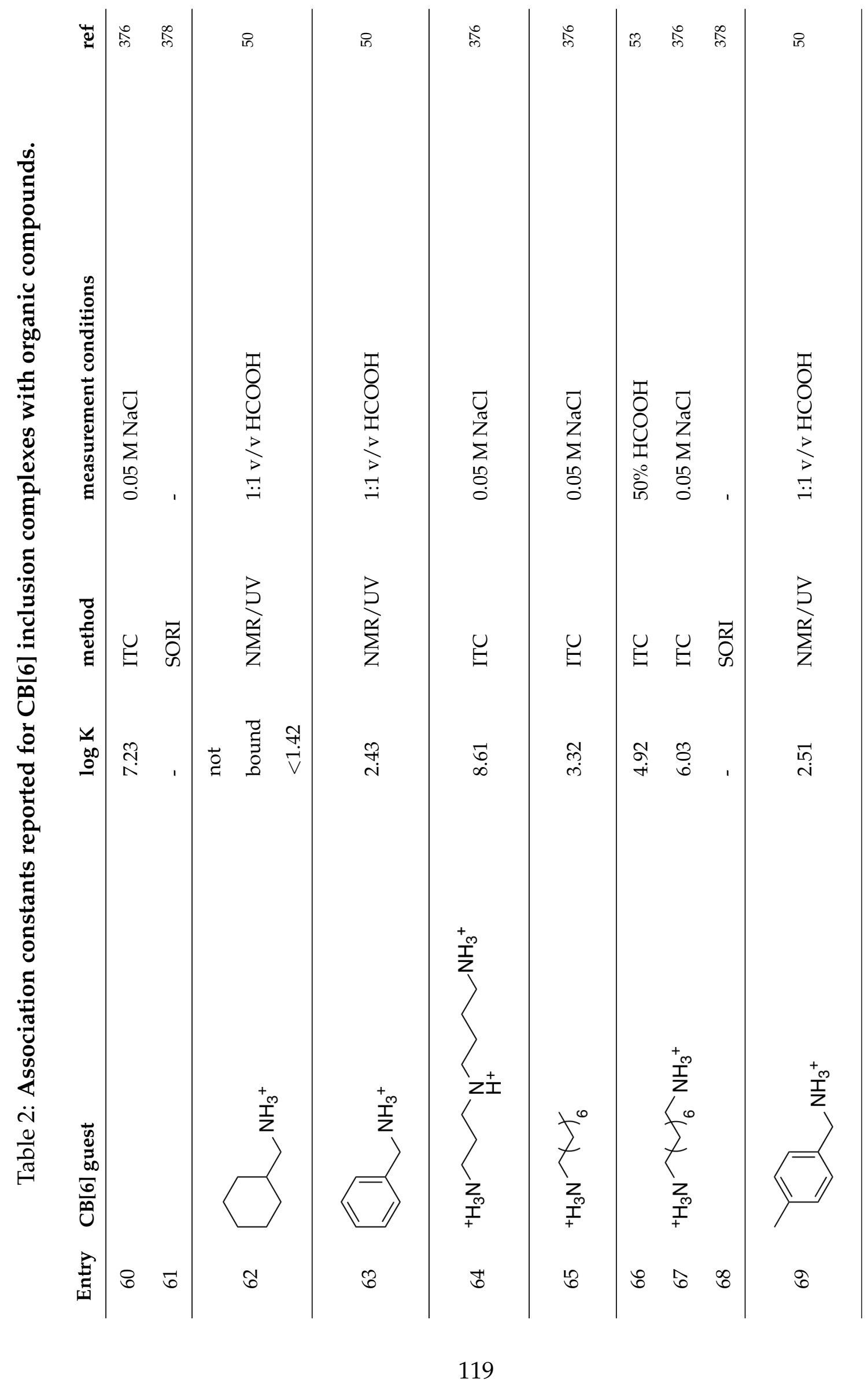




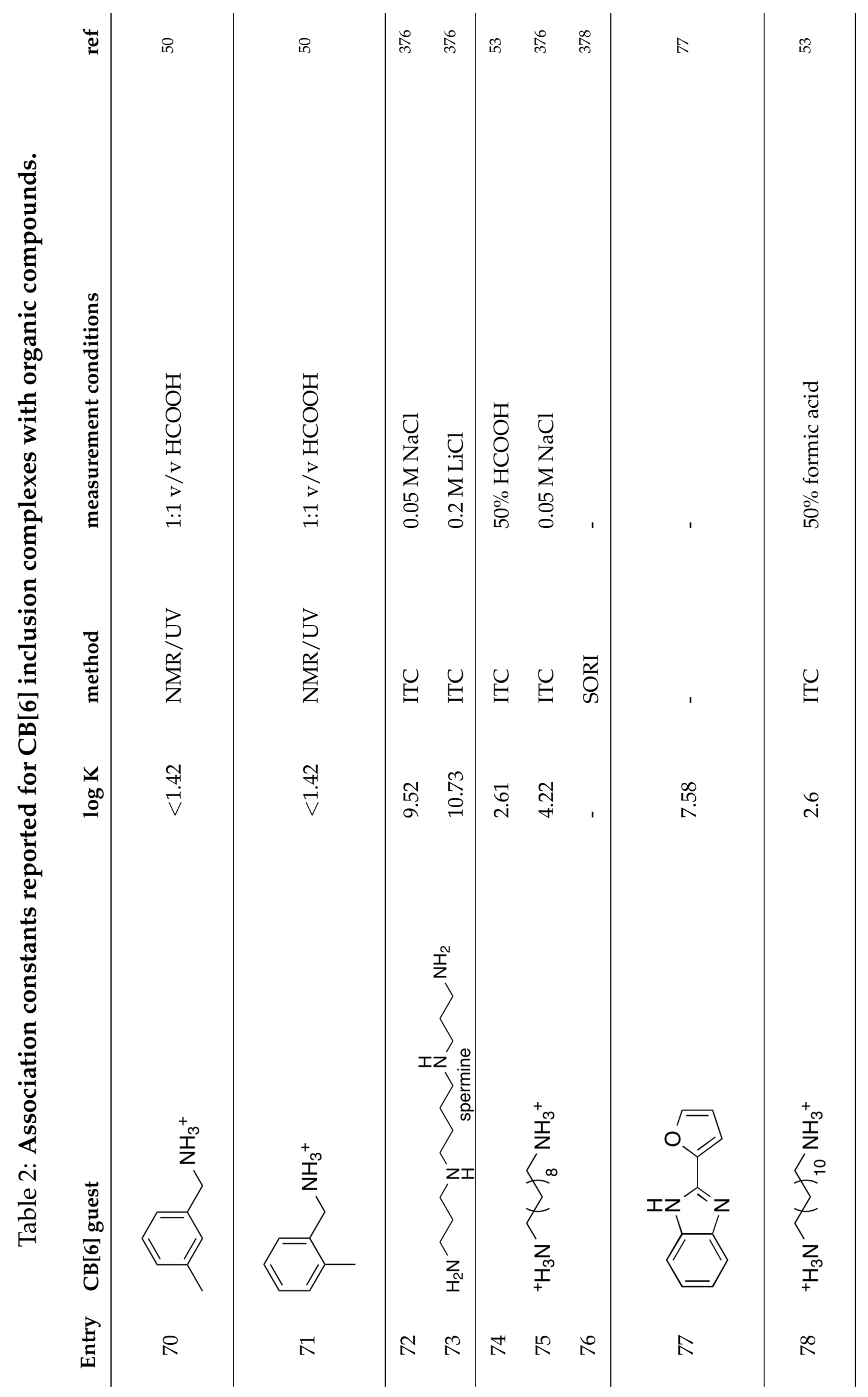




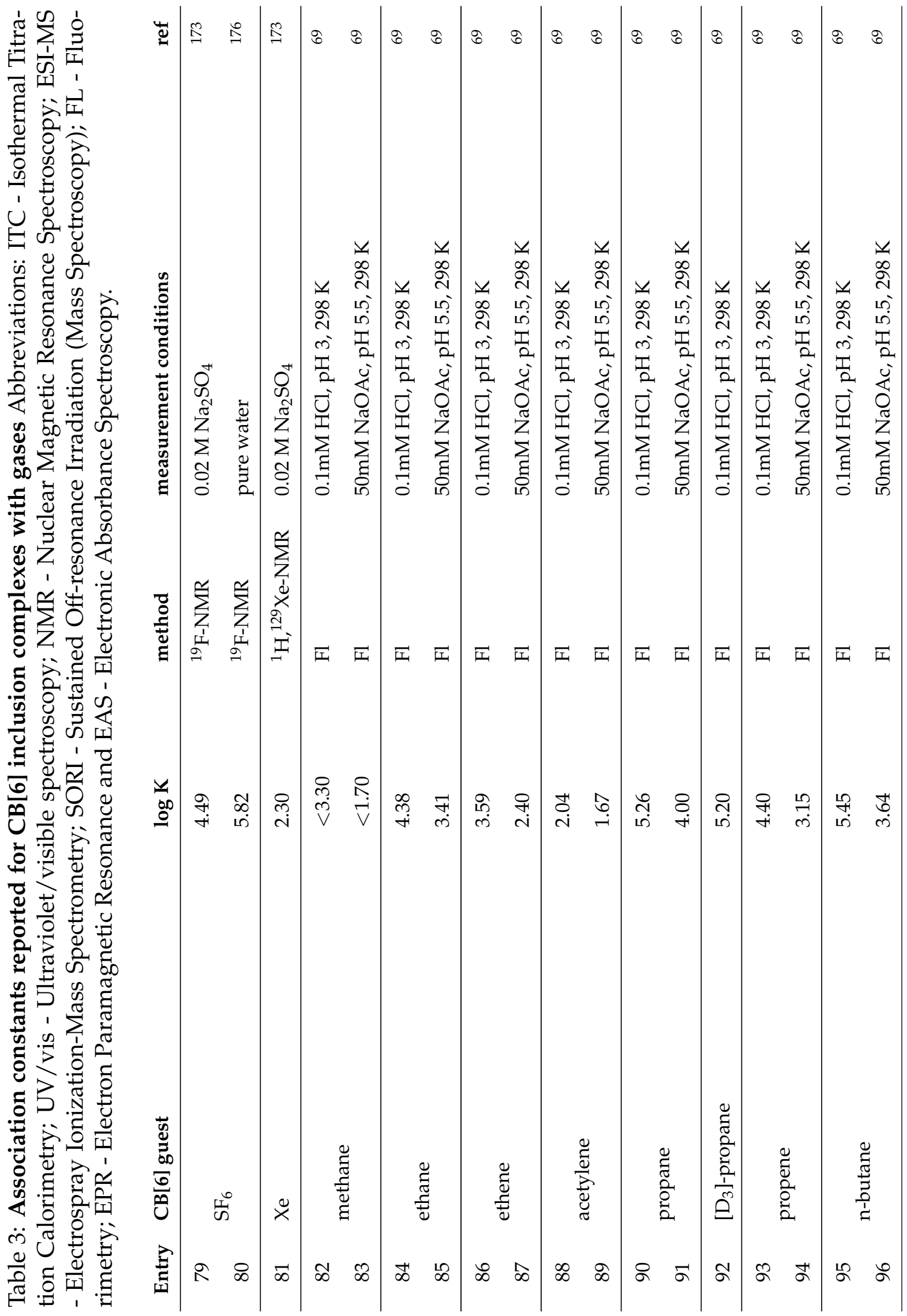




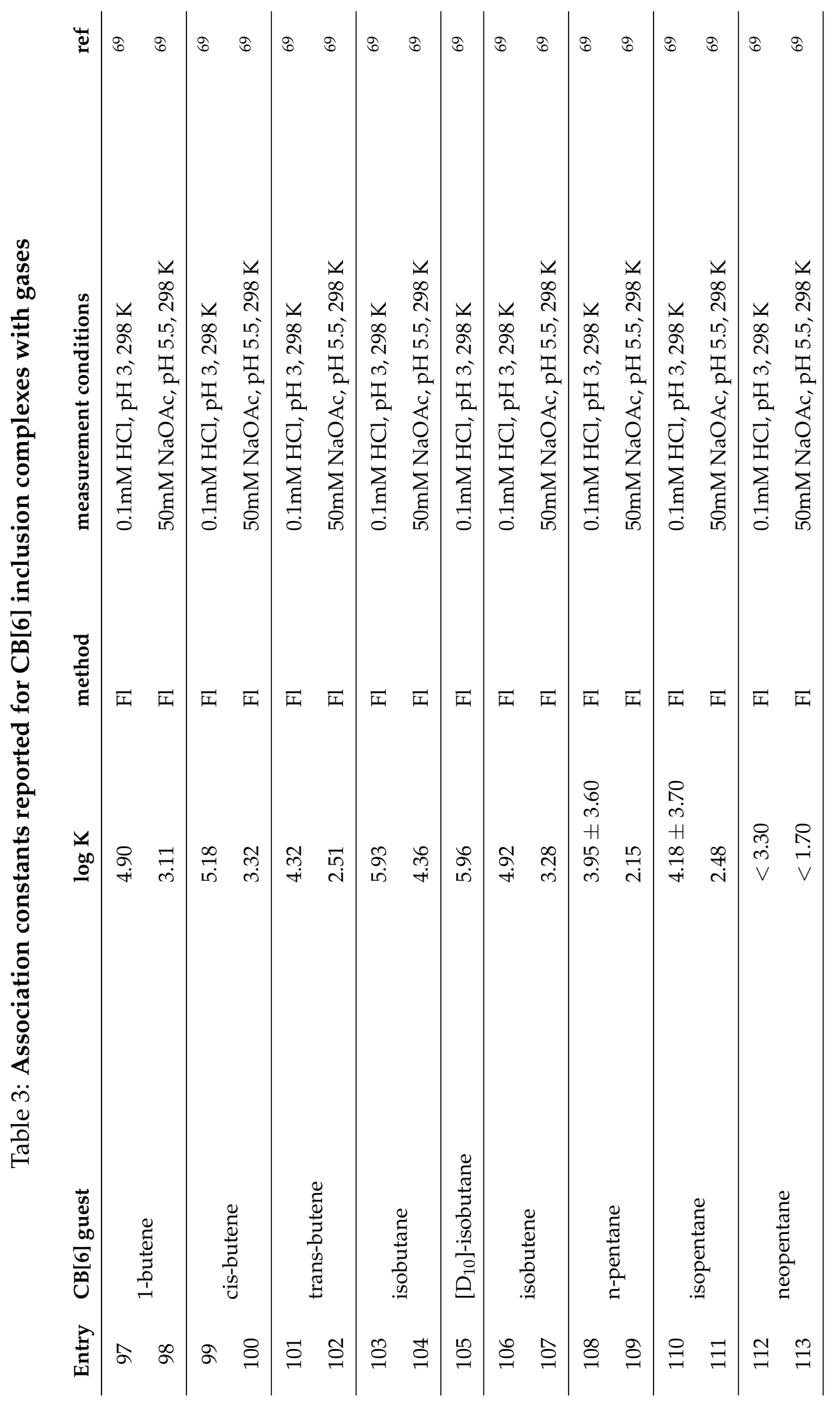




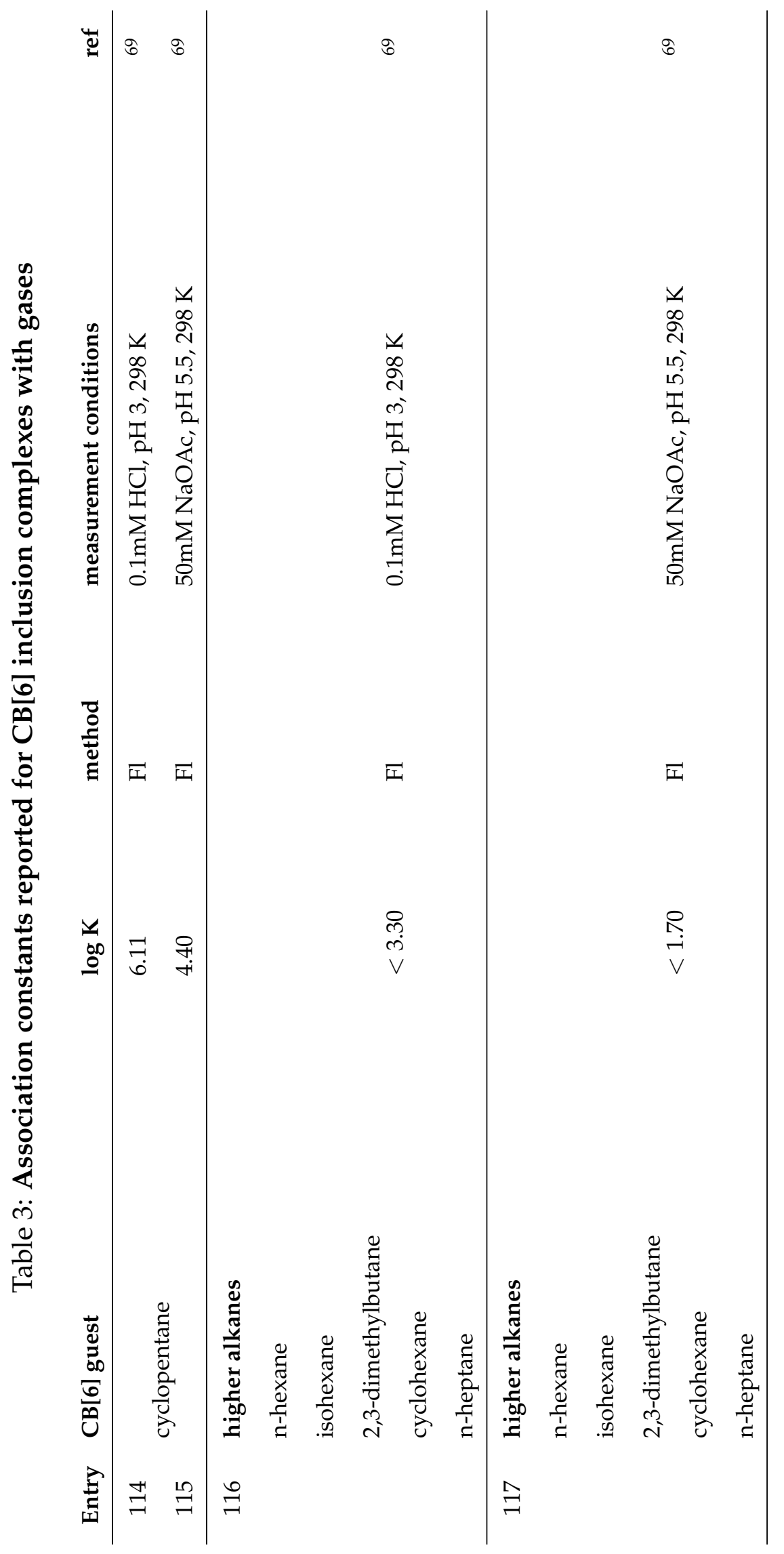




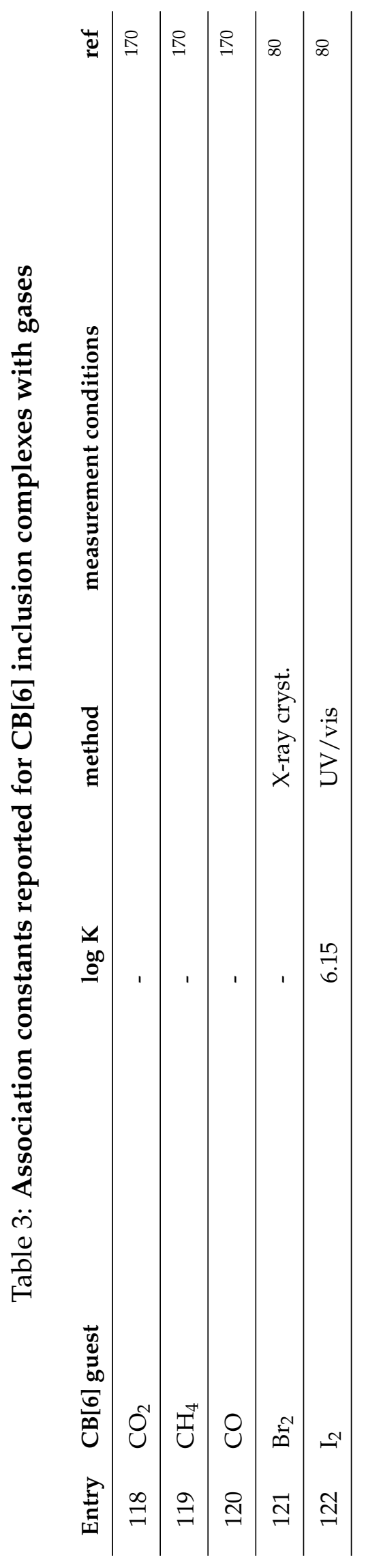




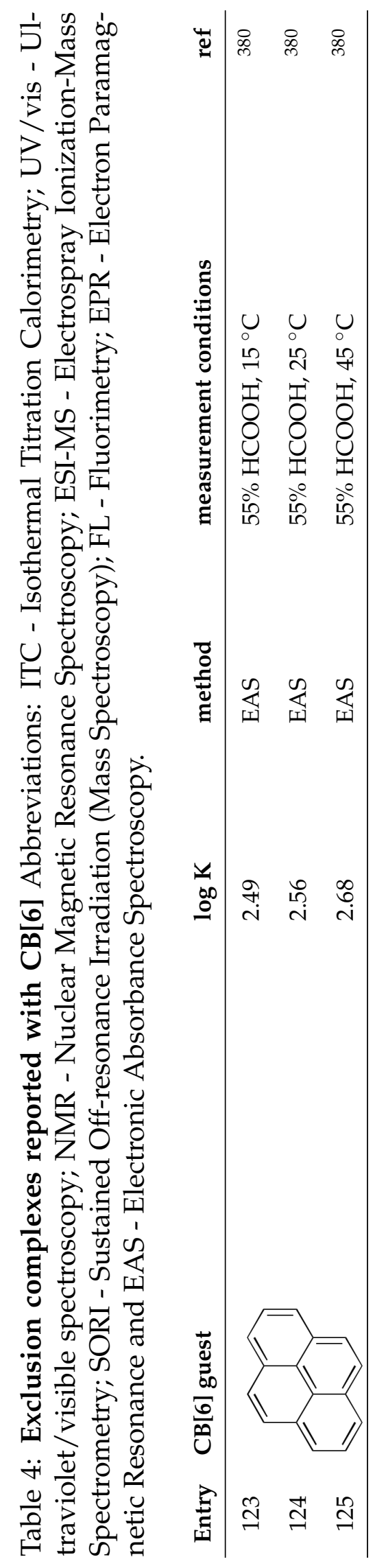

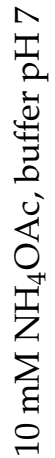

U

लै

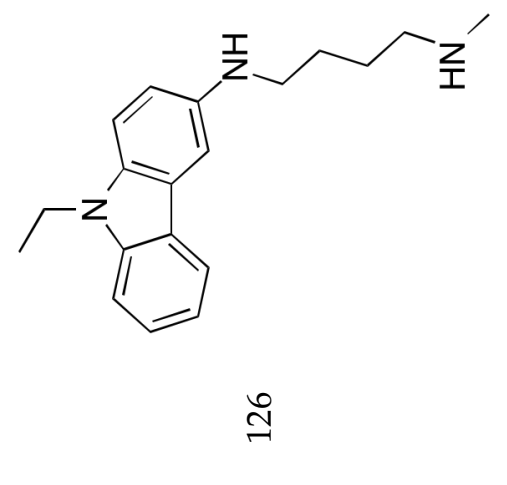




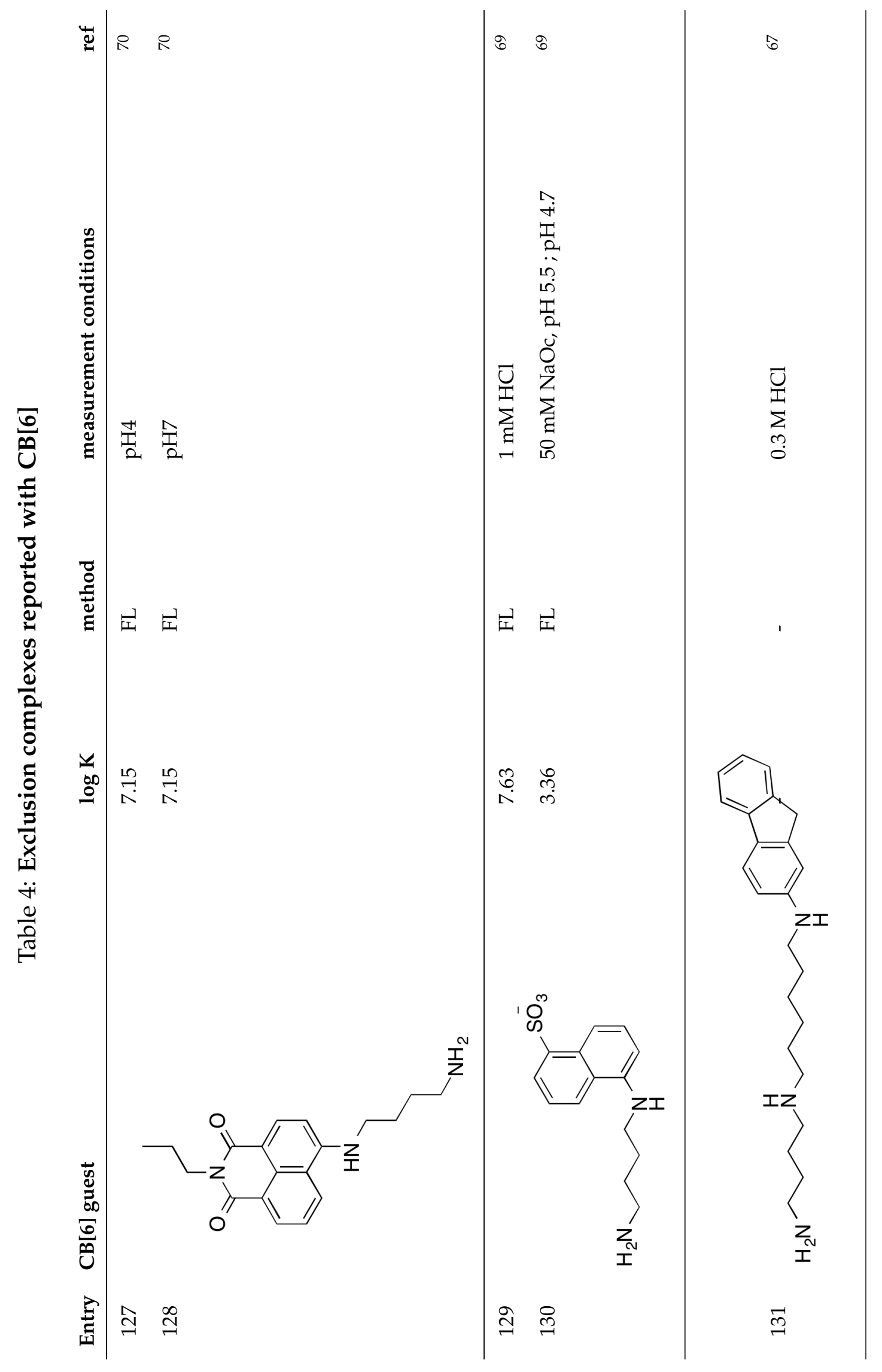




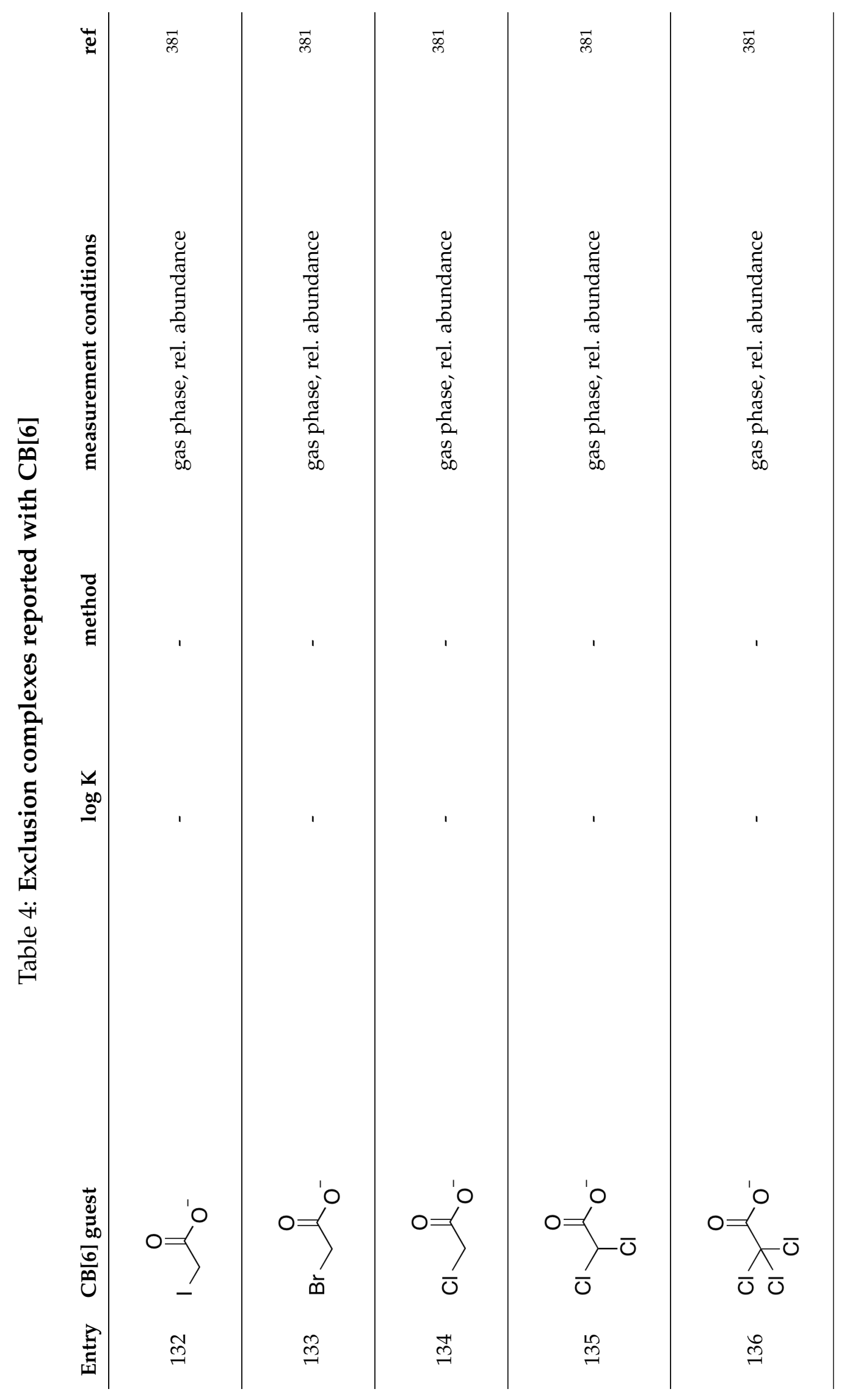



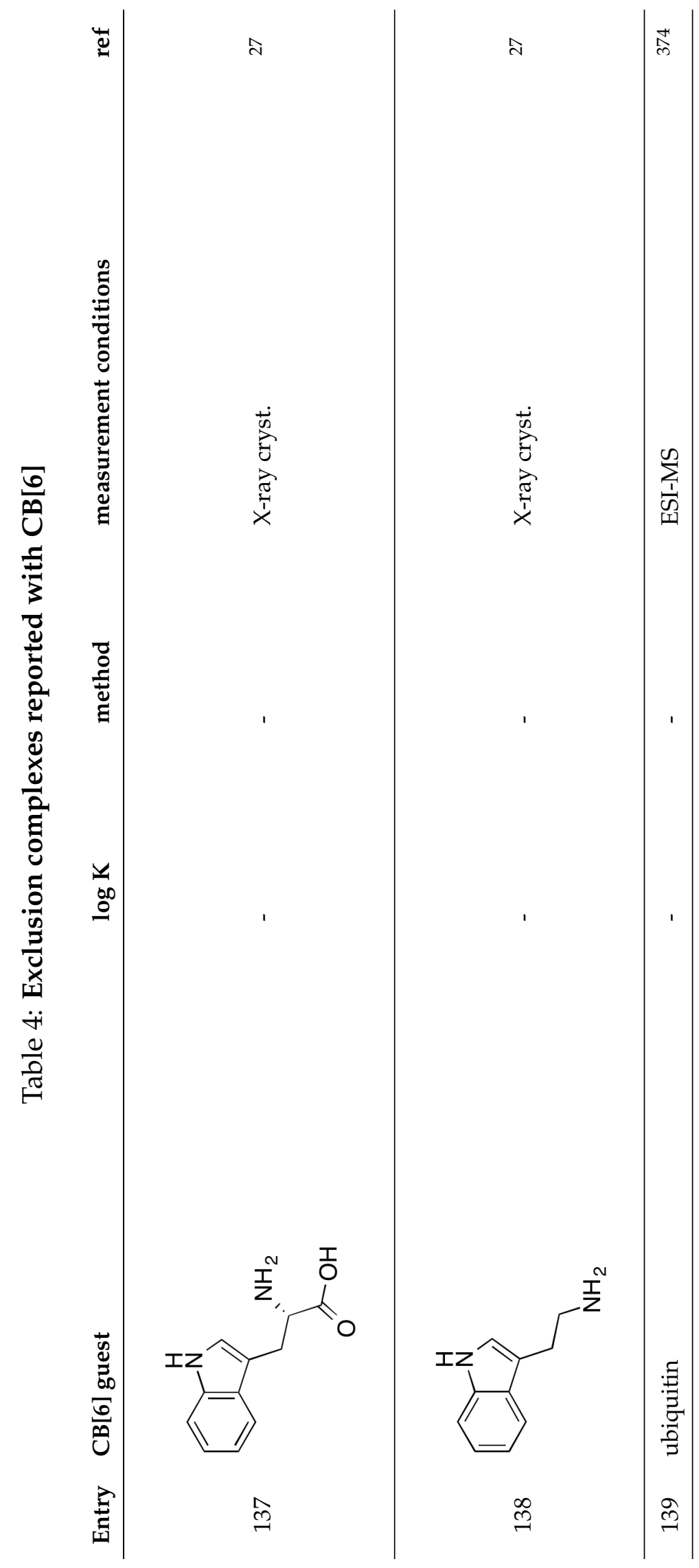

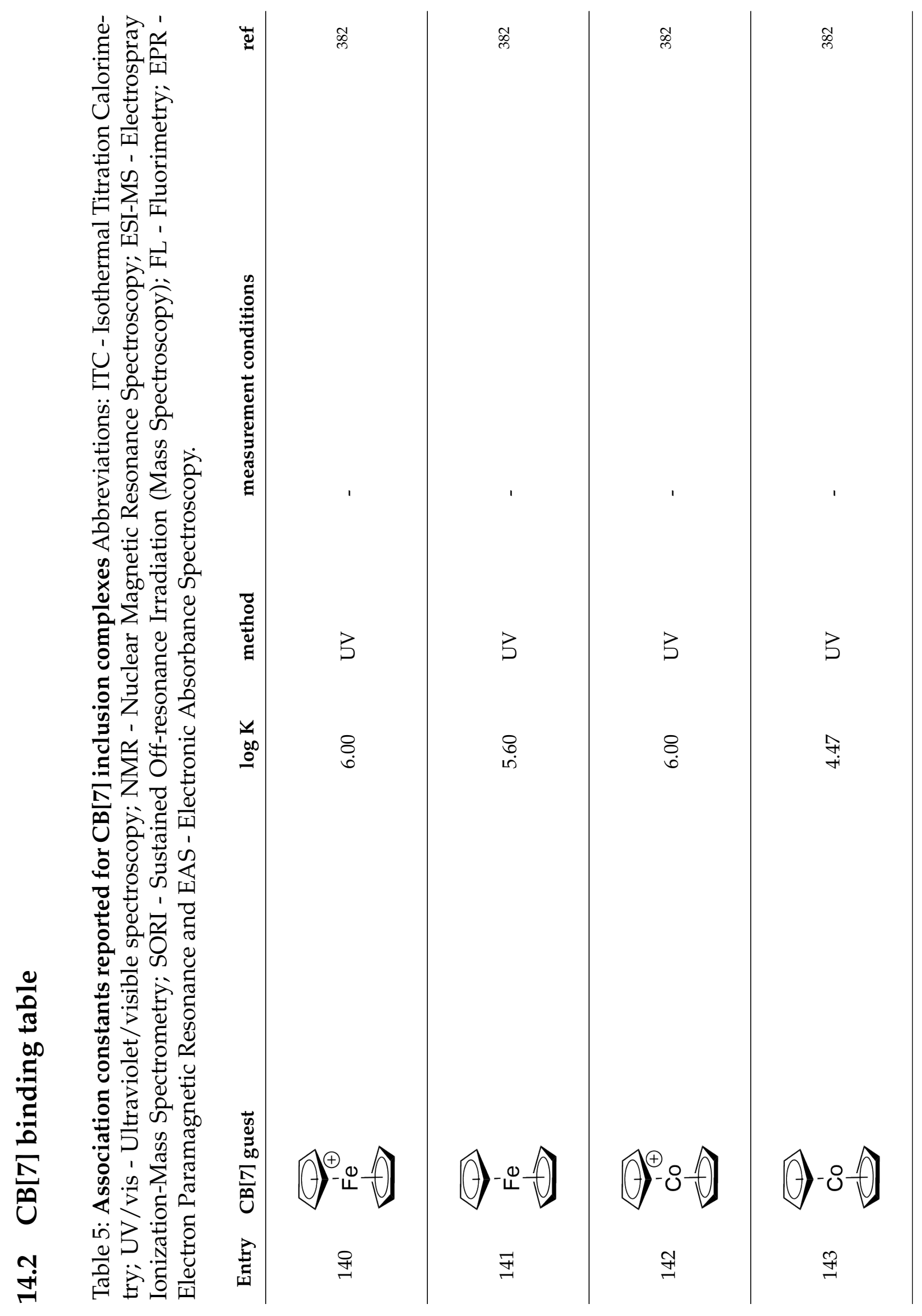


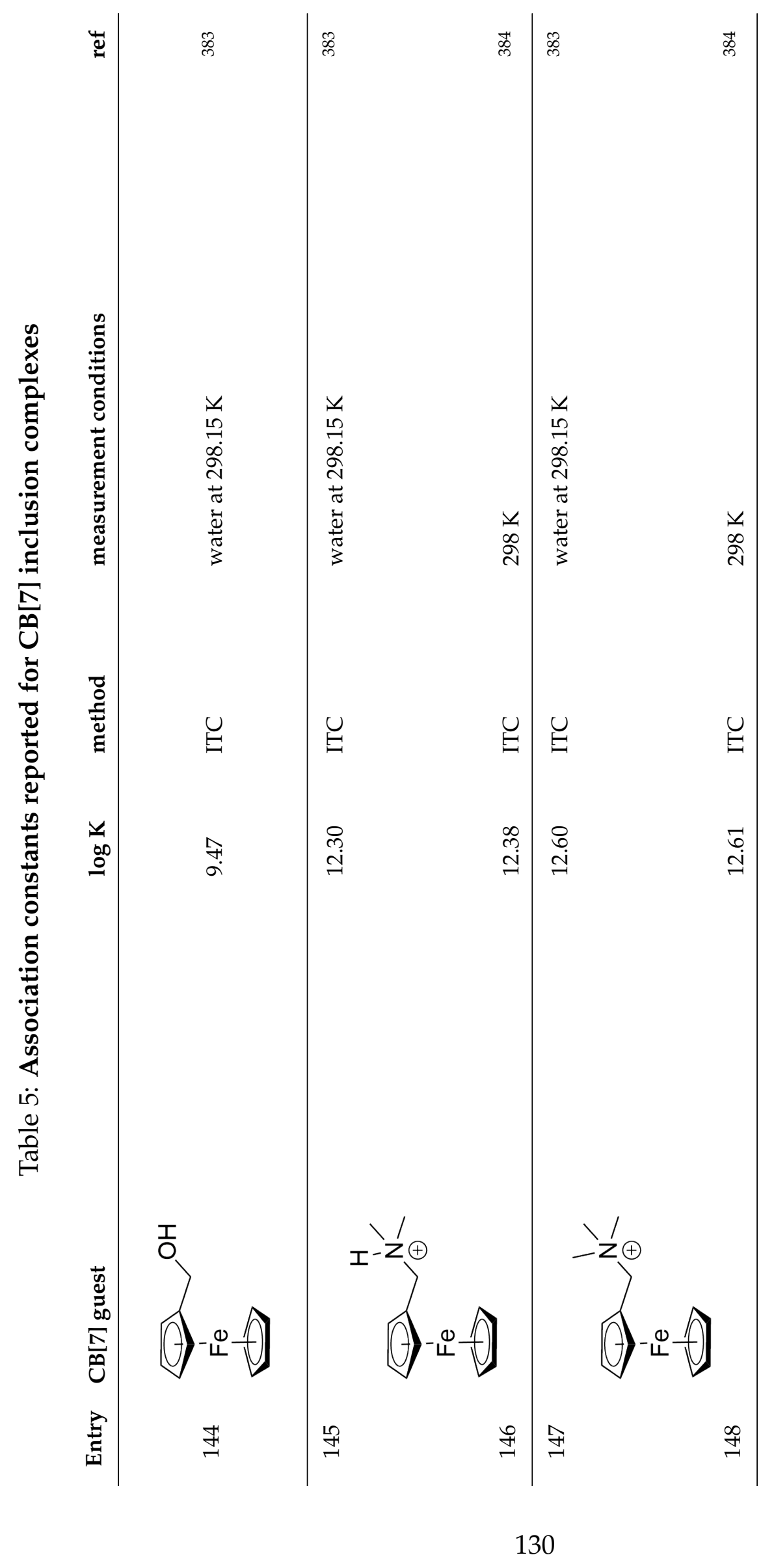




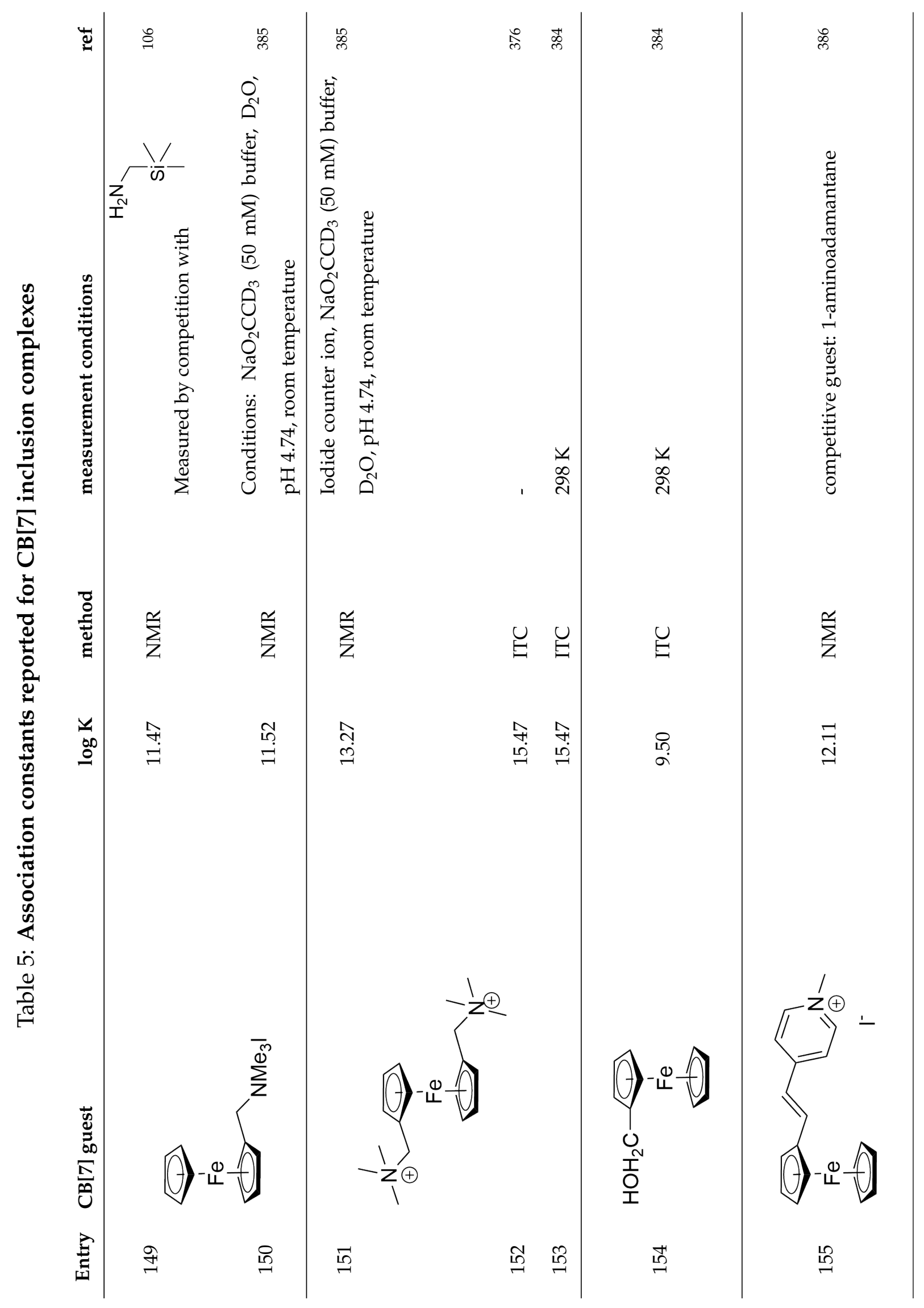




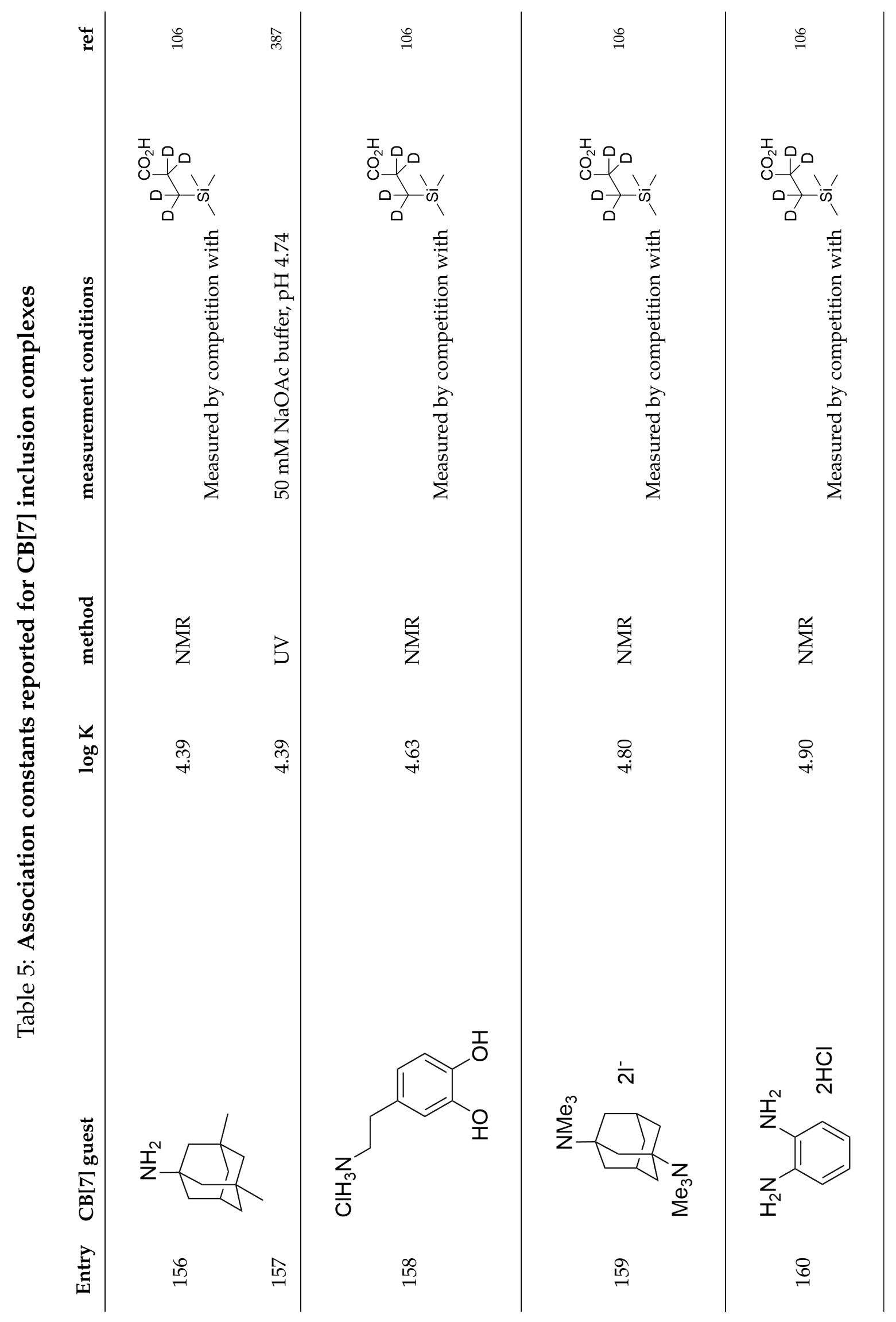




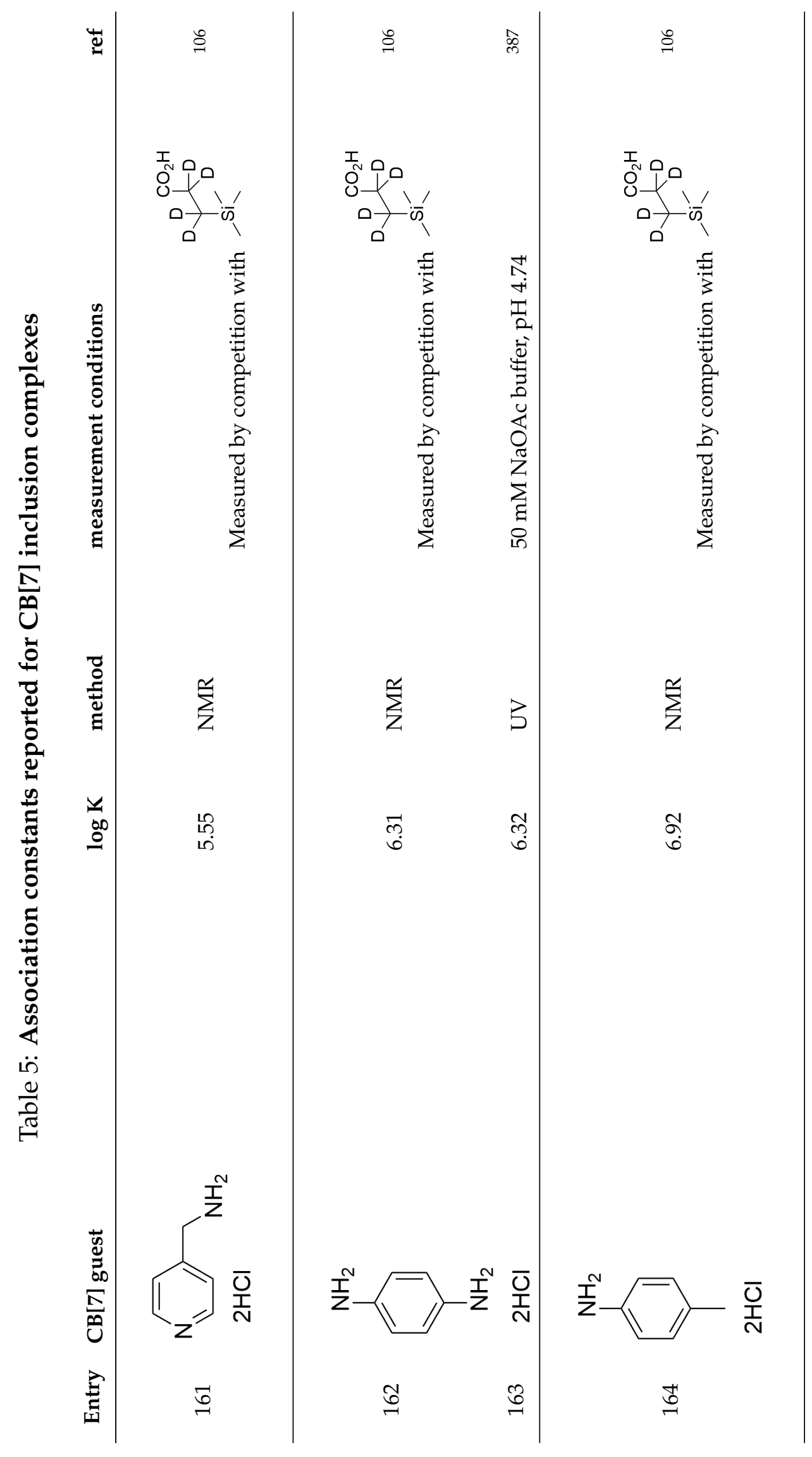




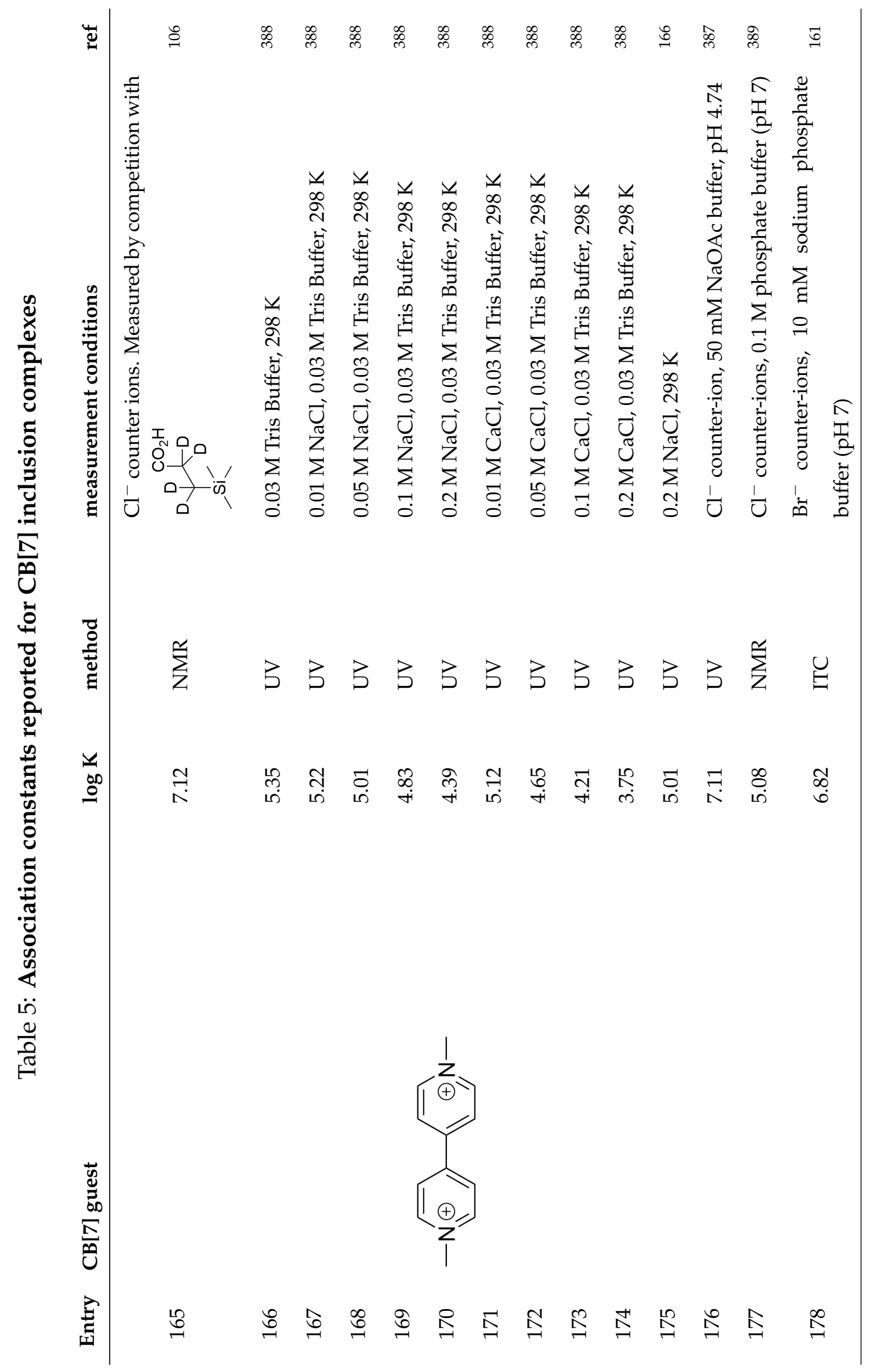



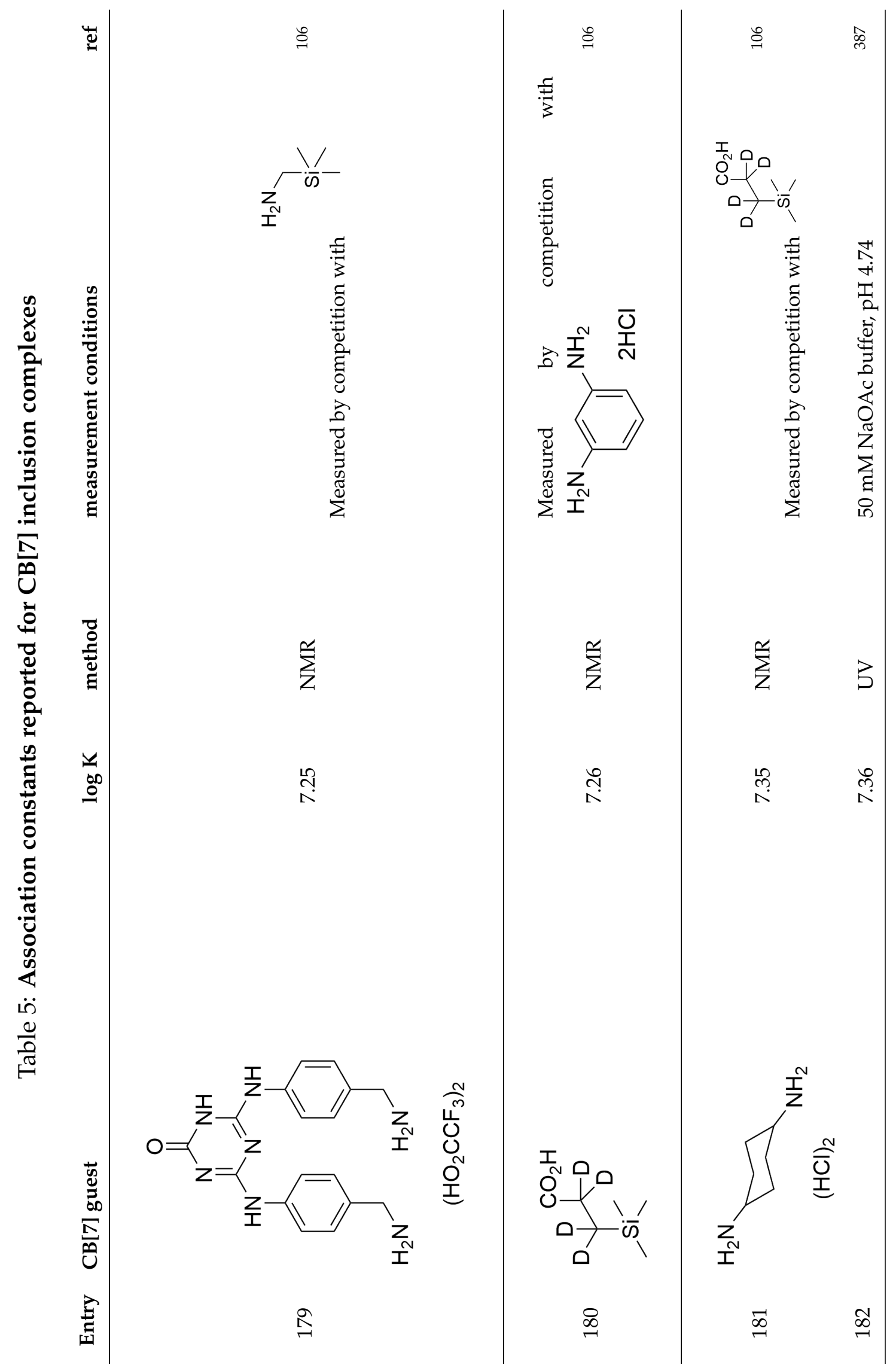


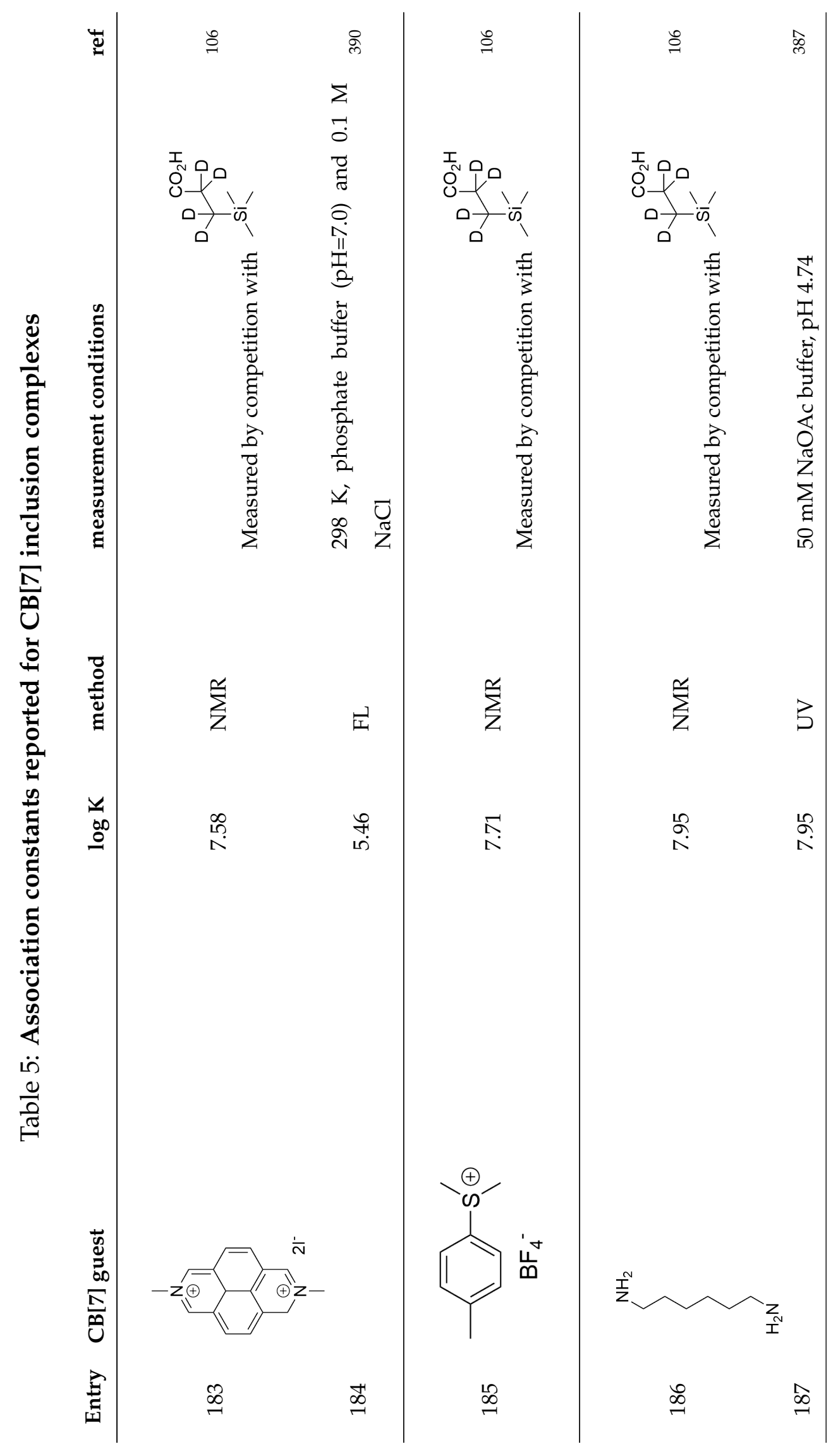



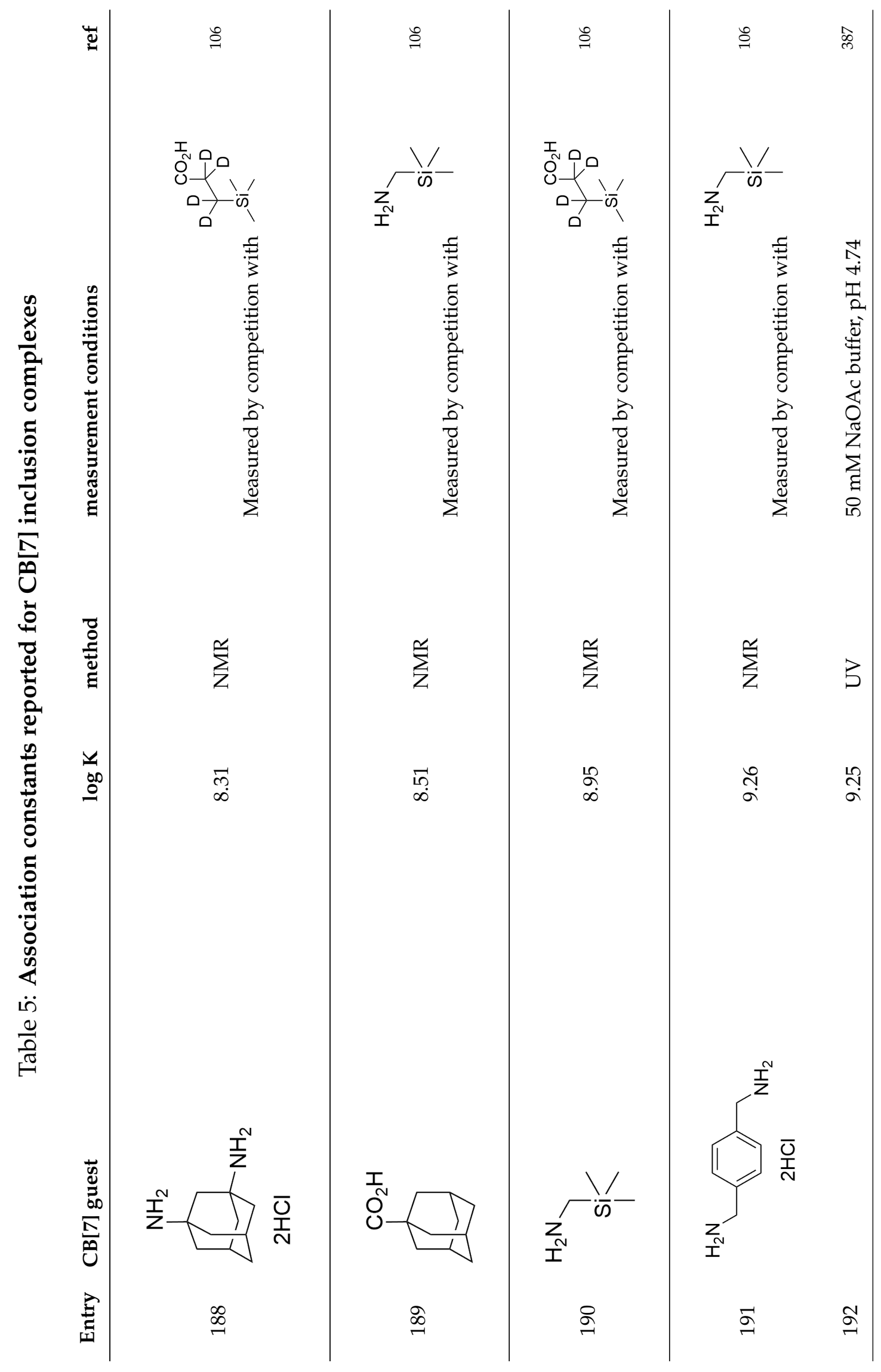

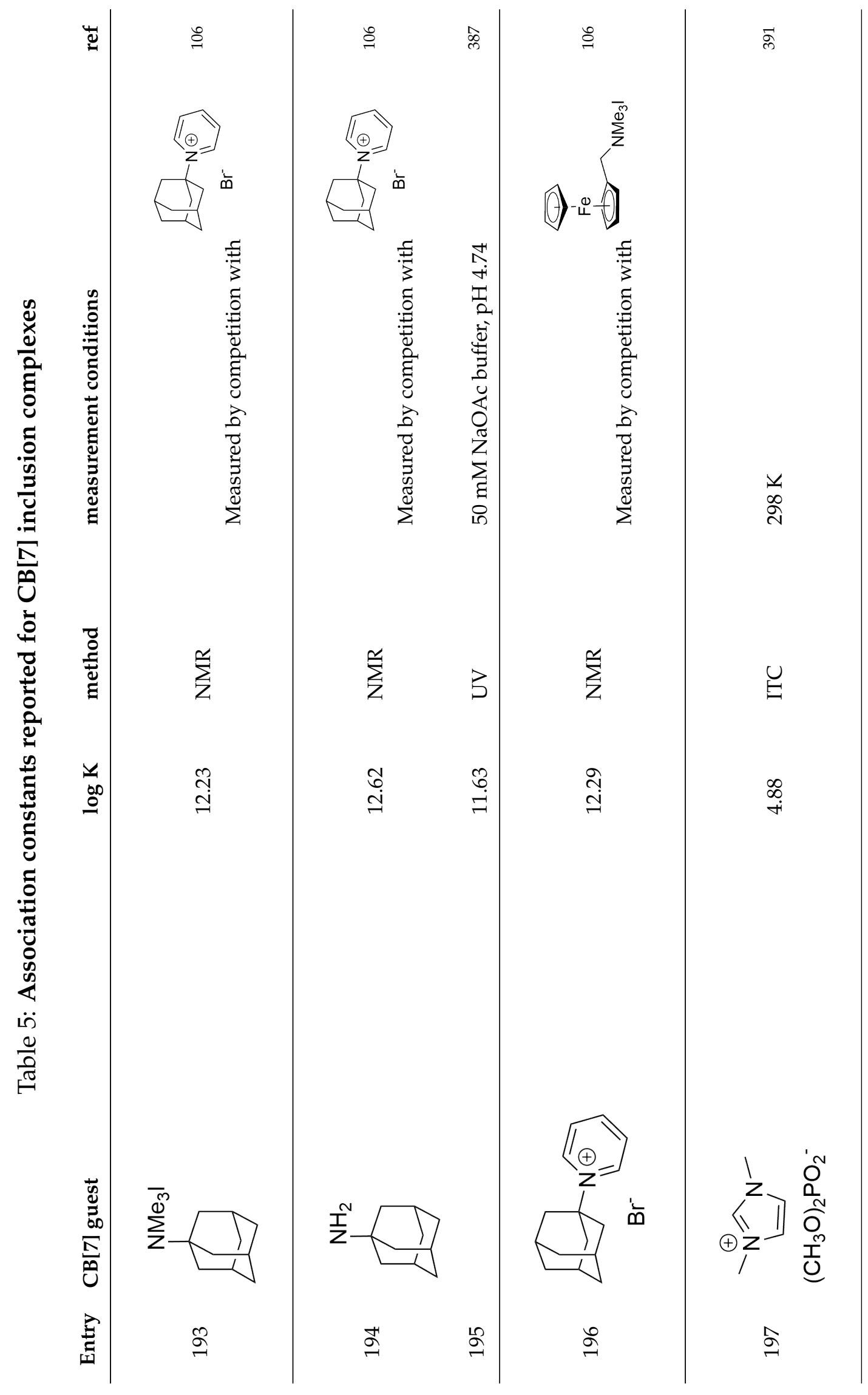

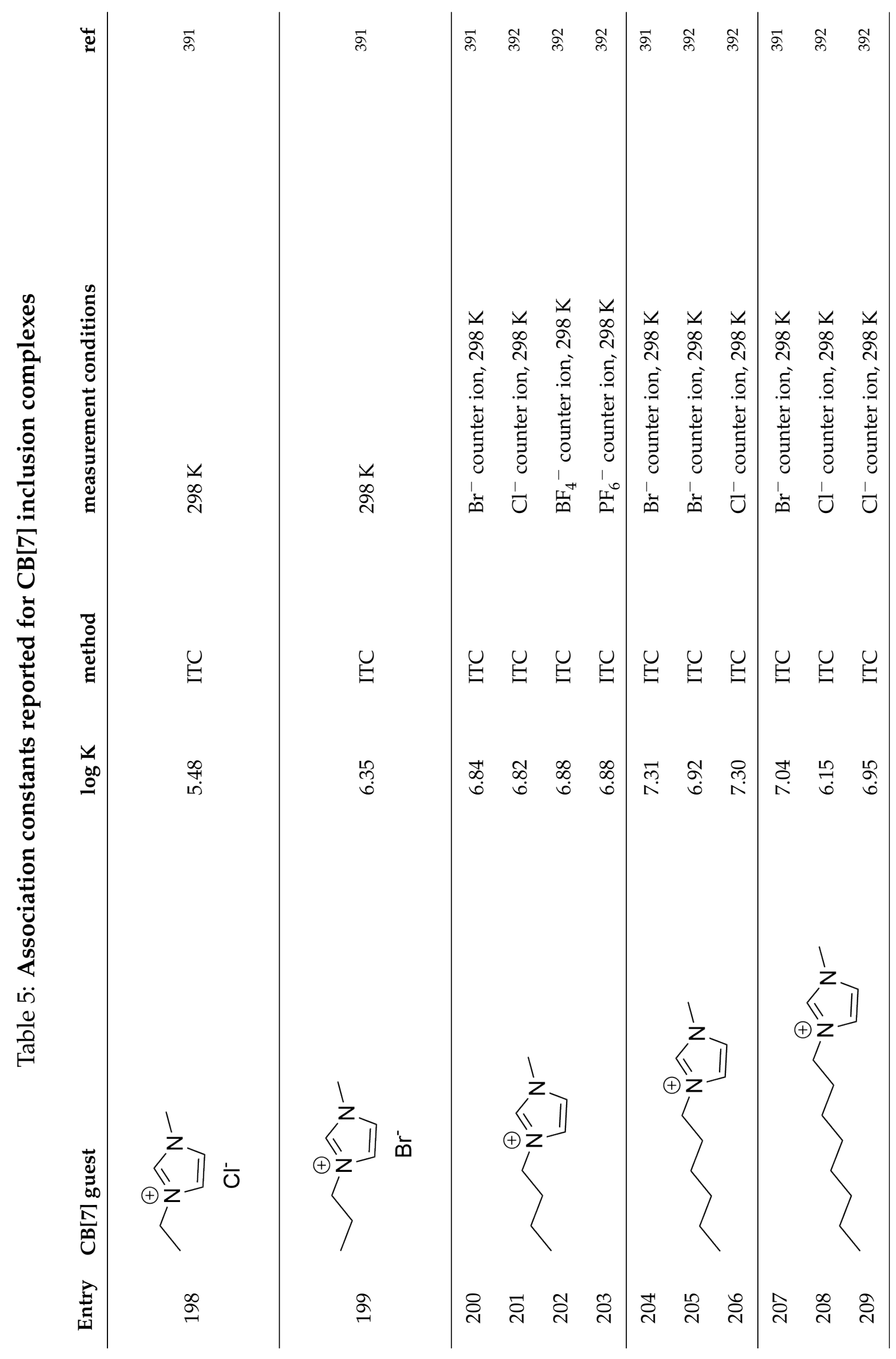


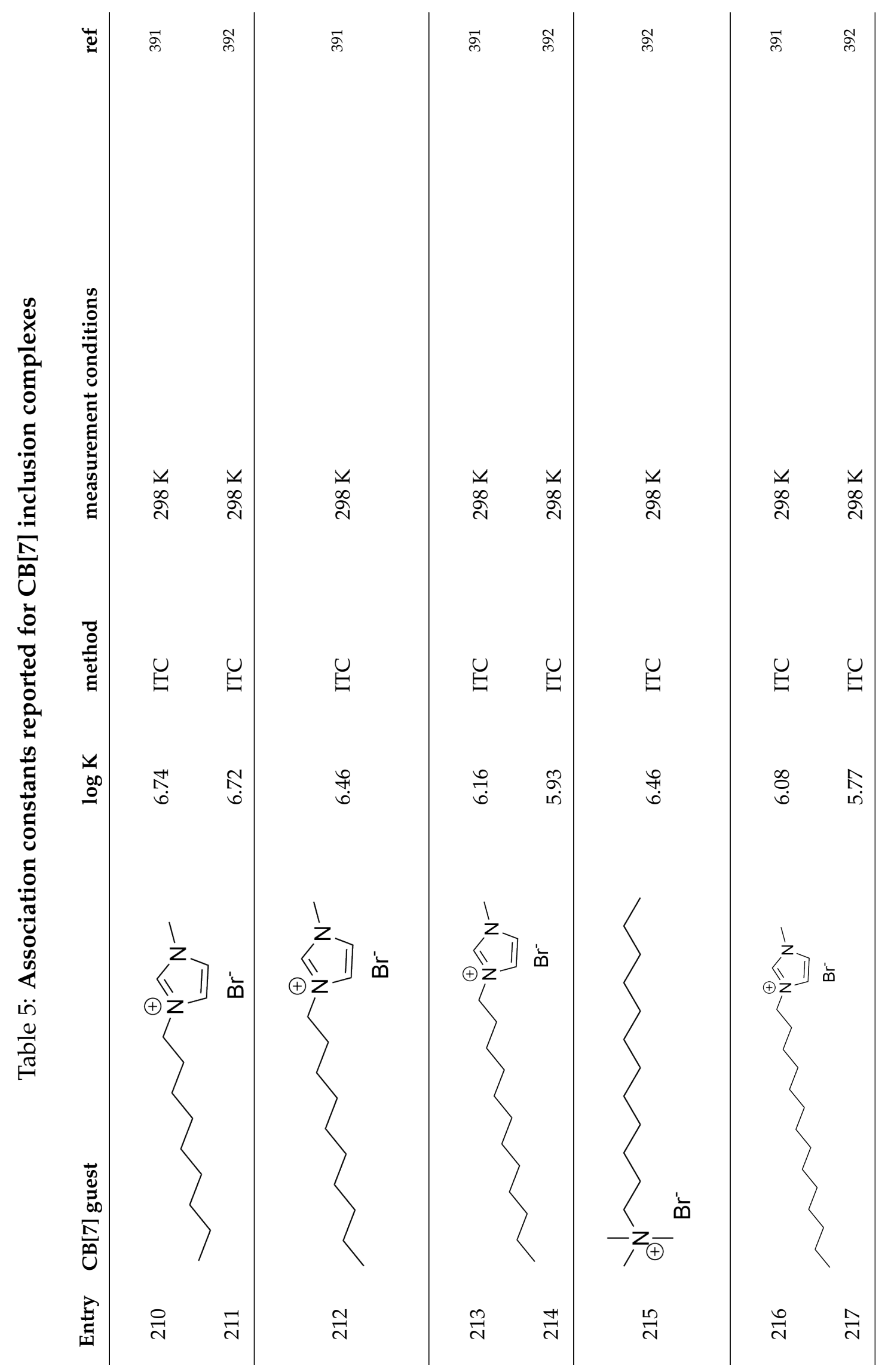




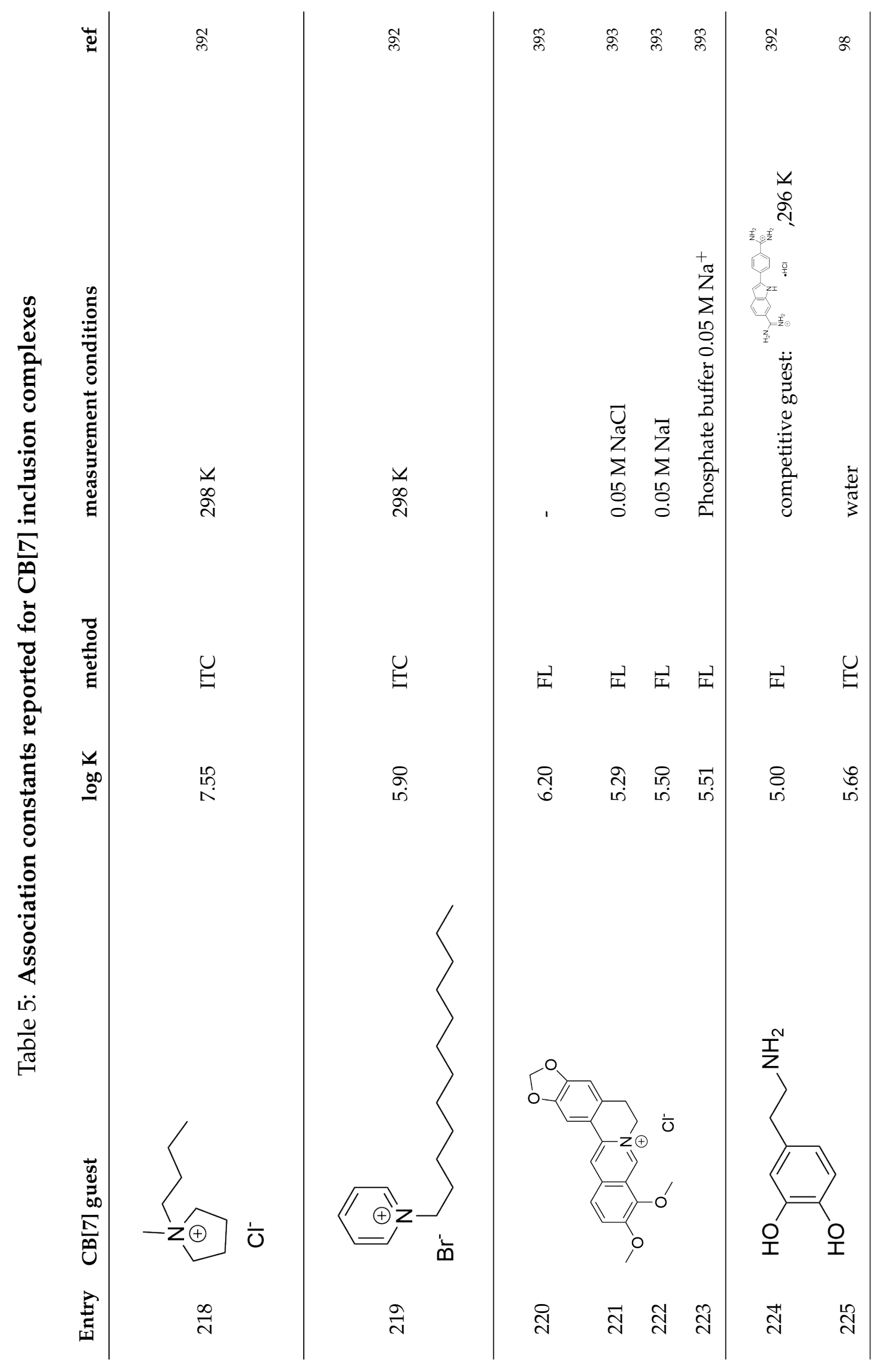



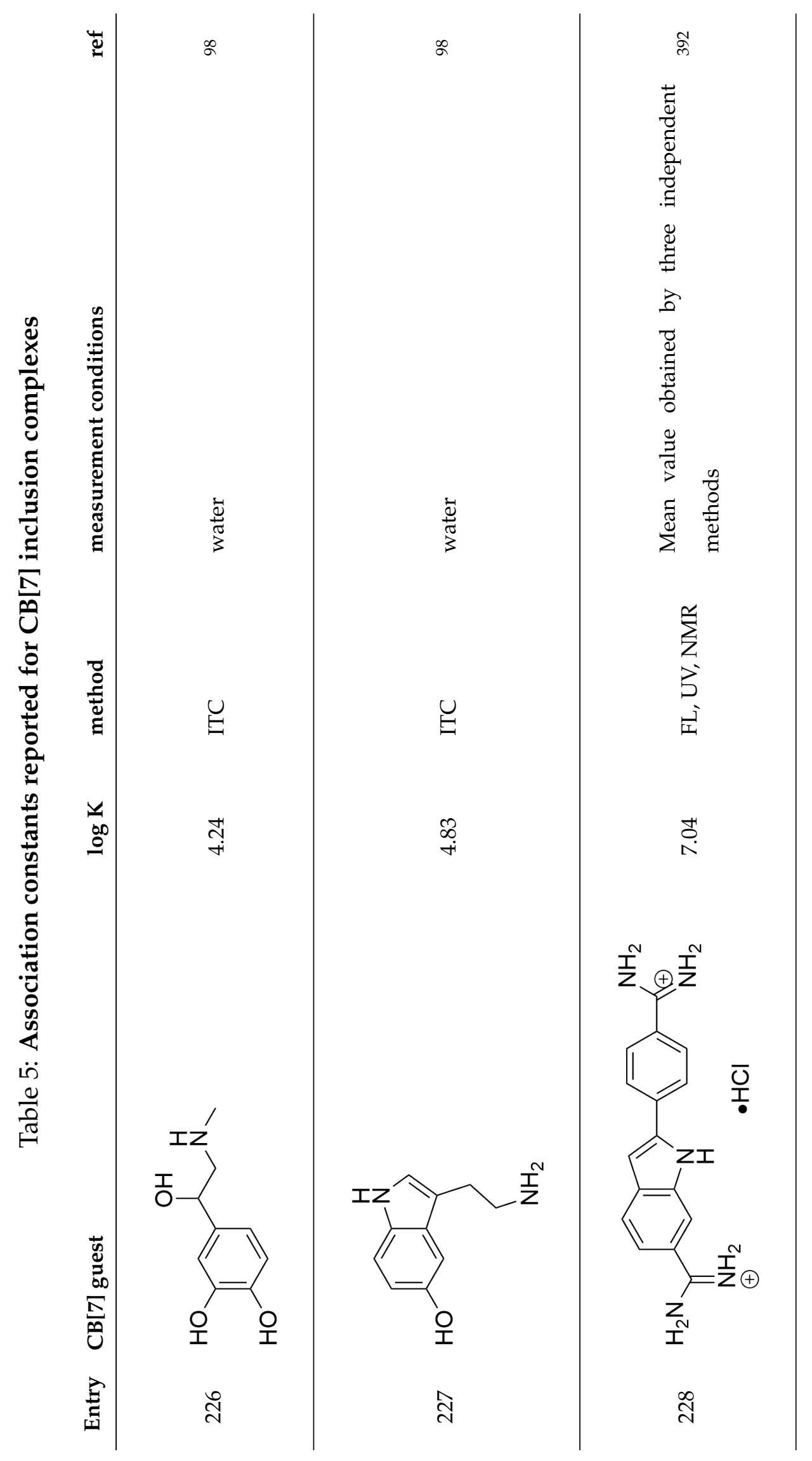


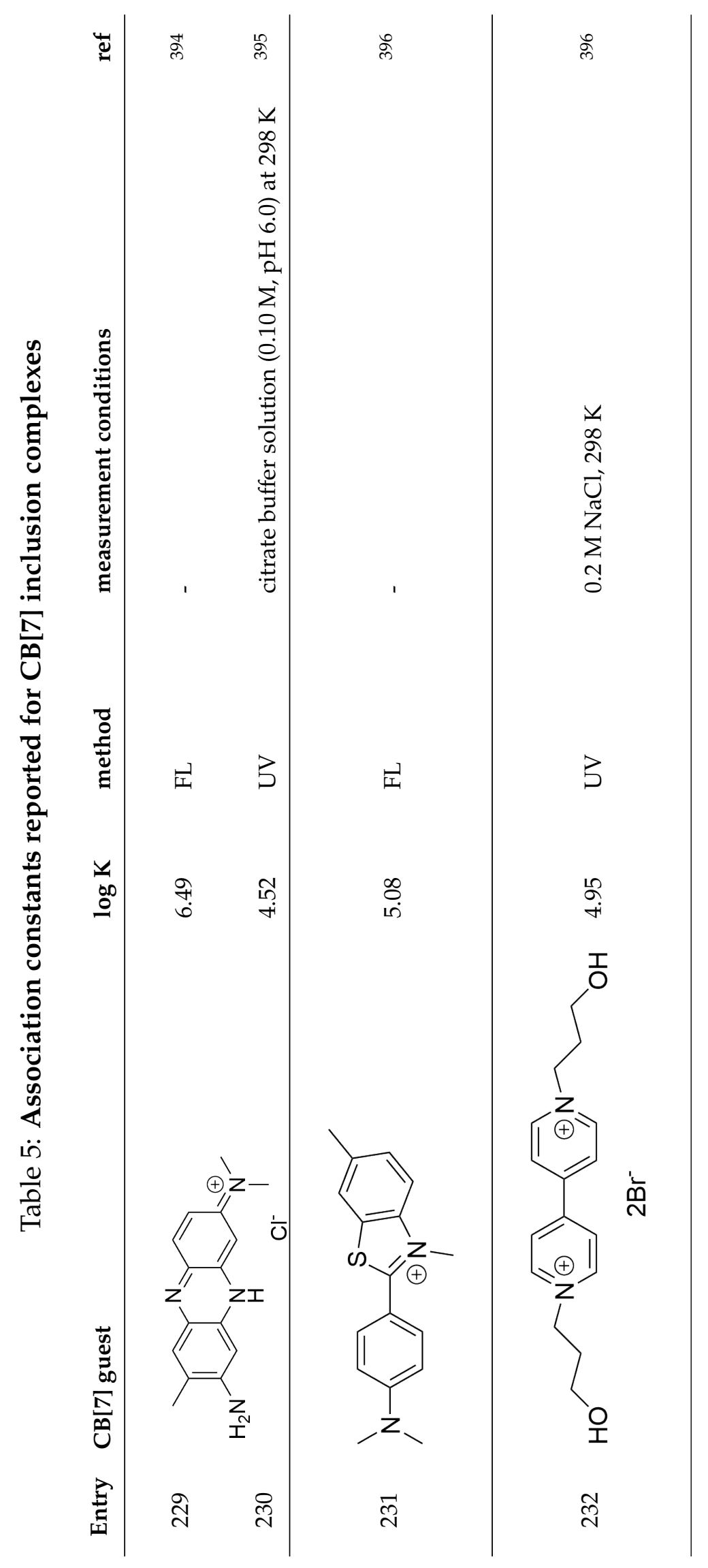




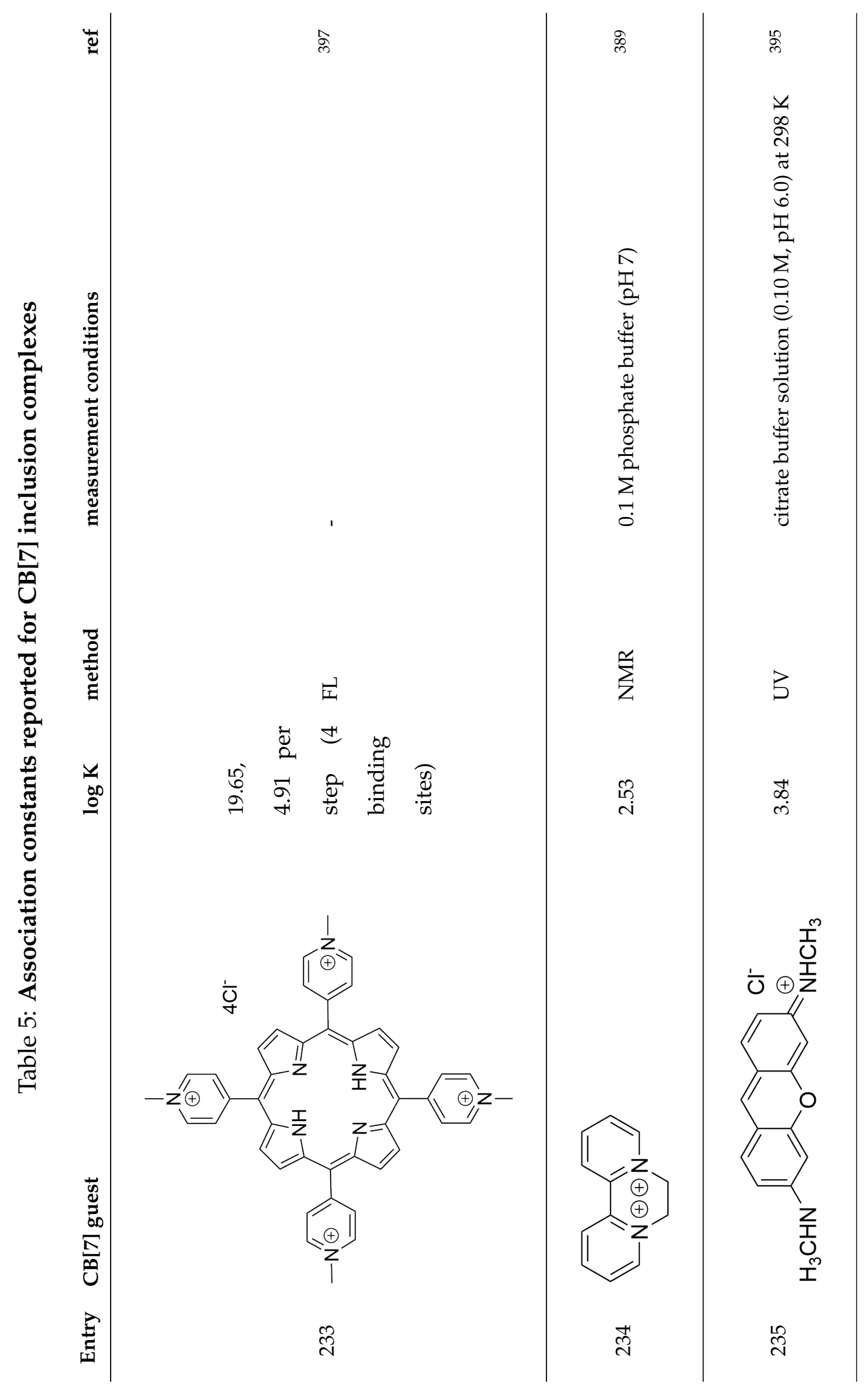




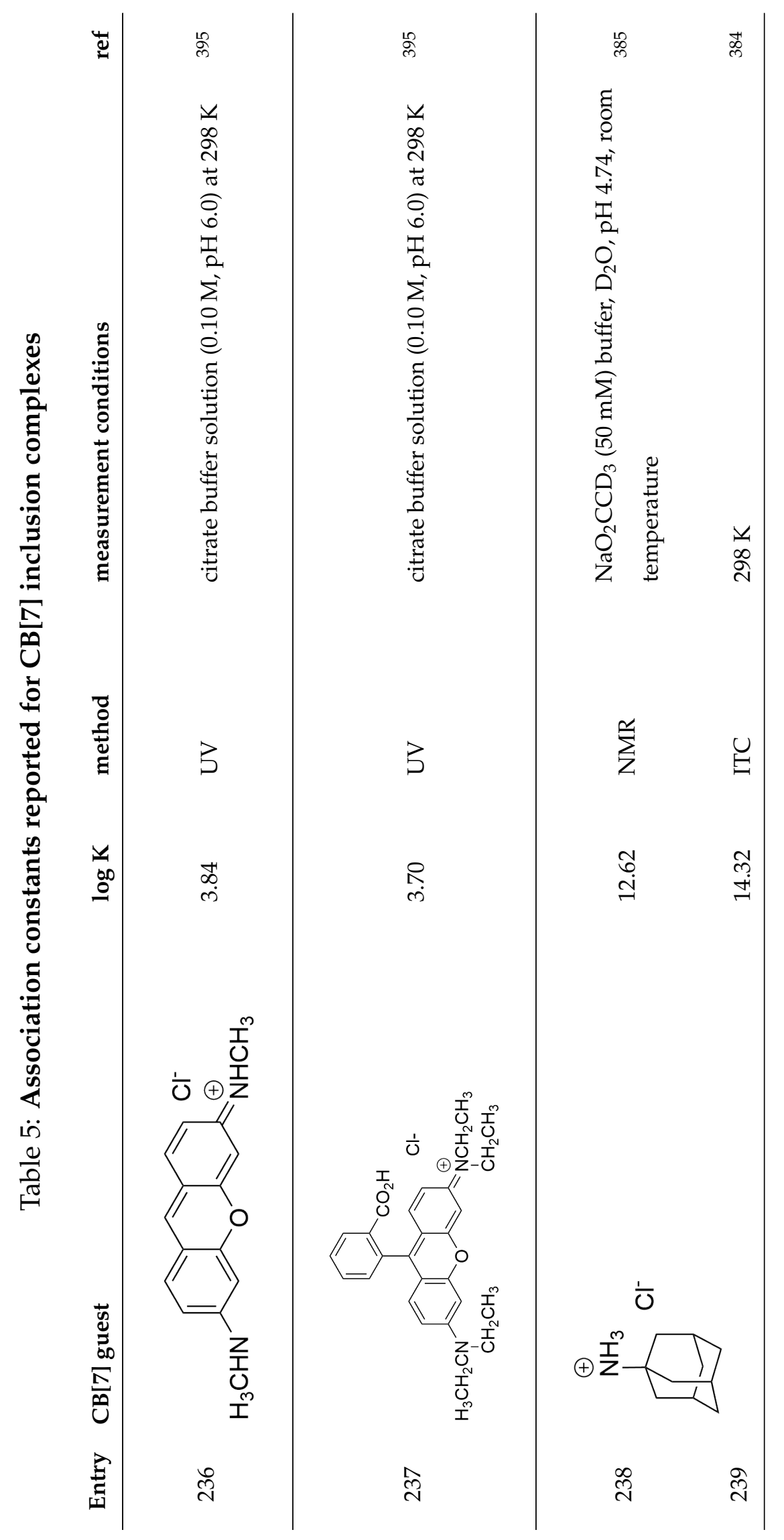




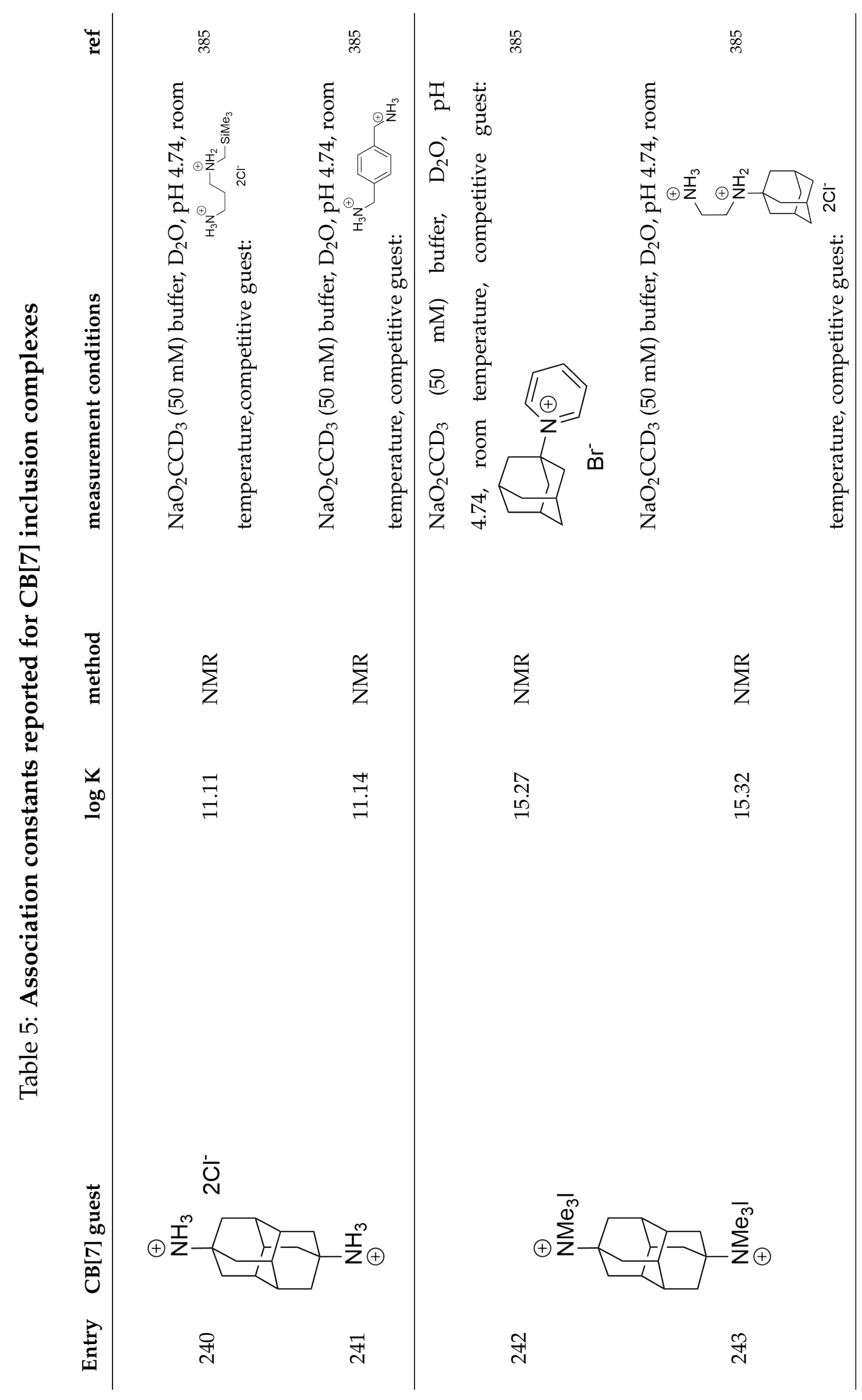



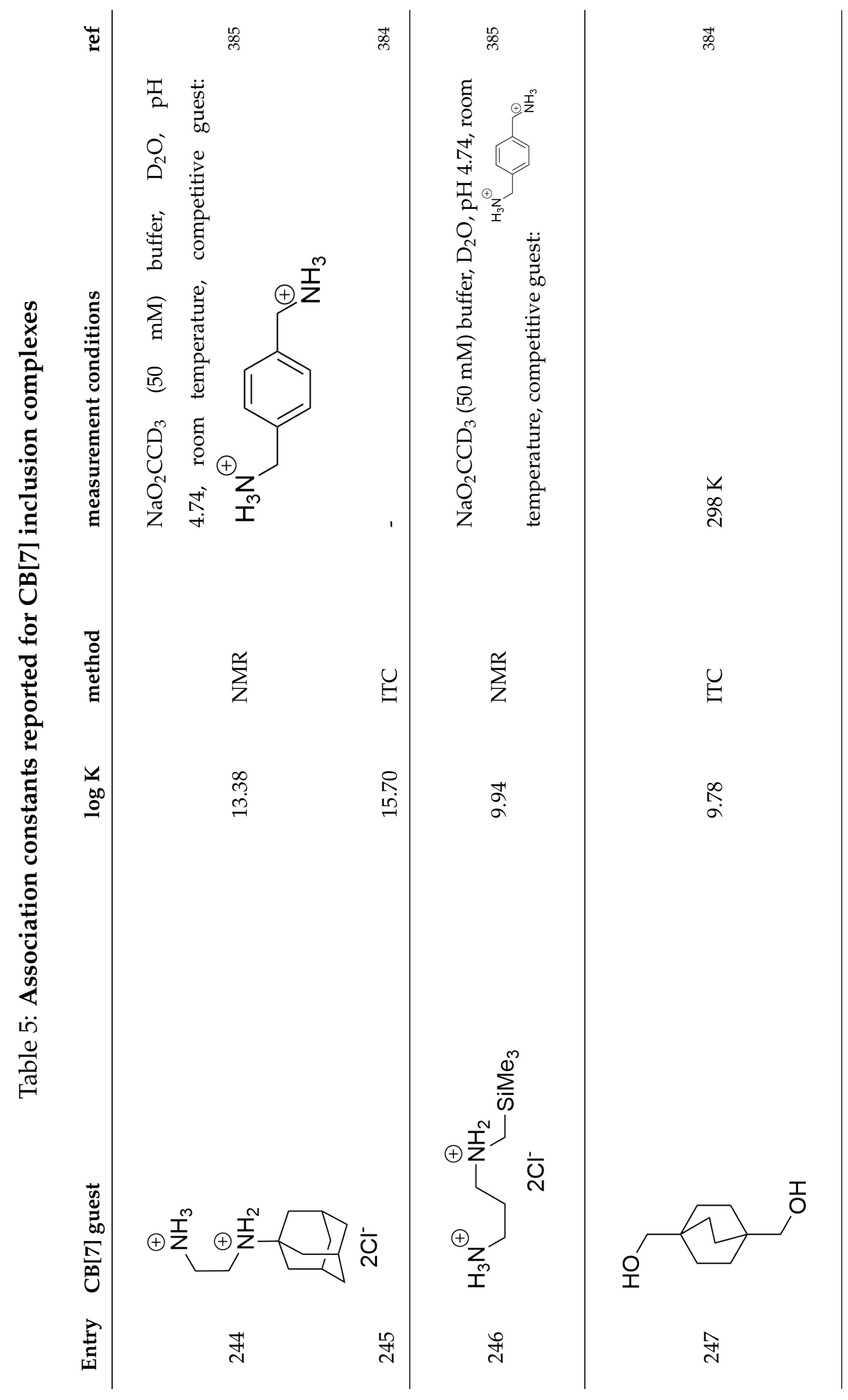

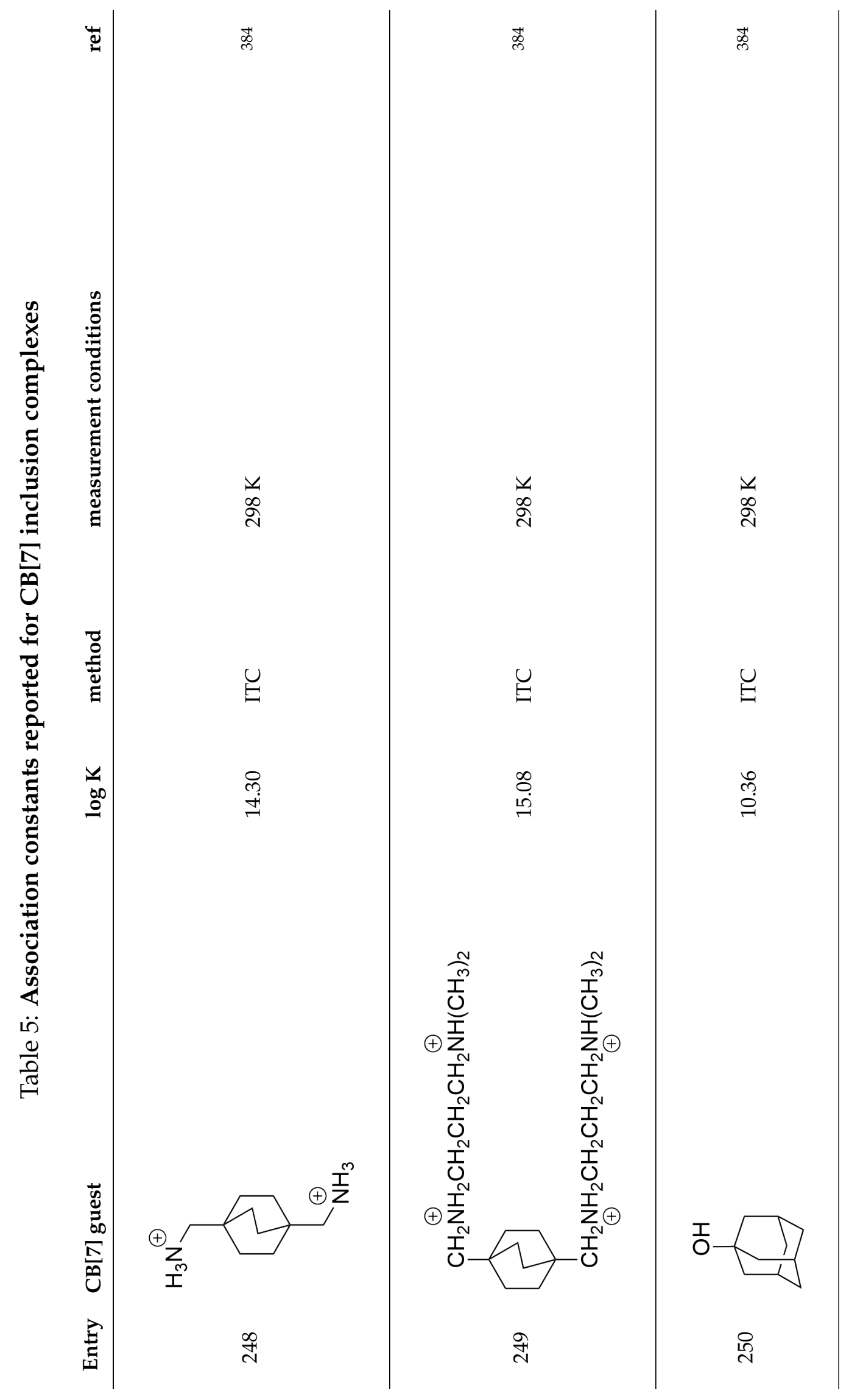

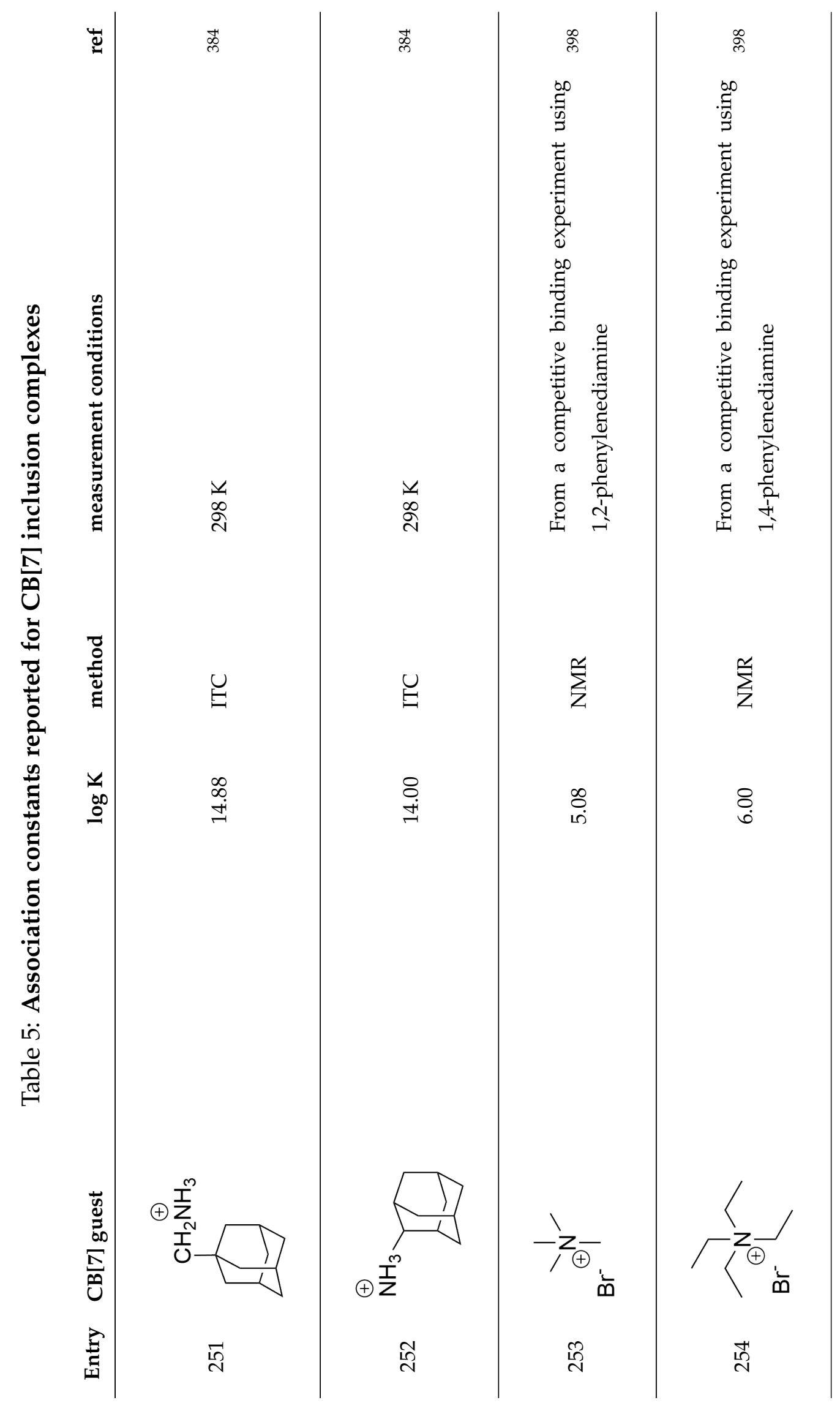

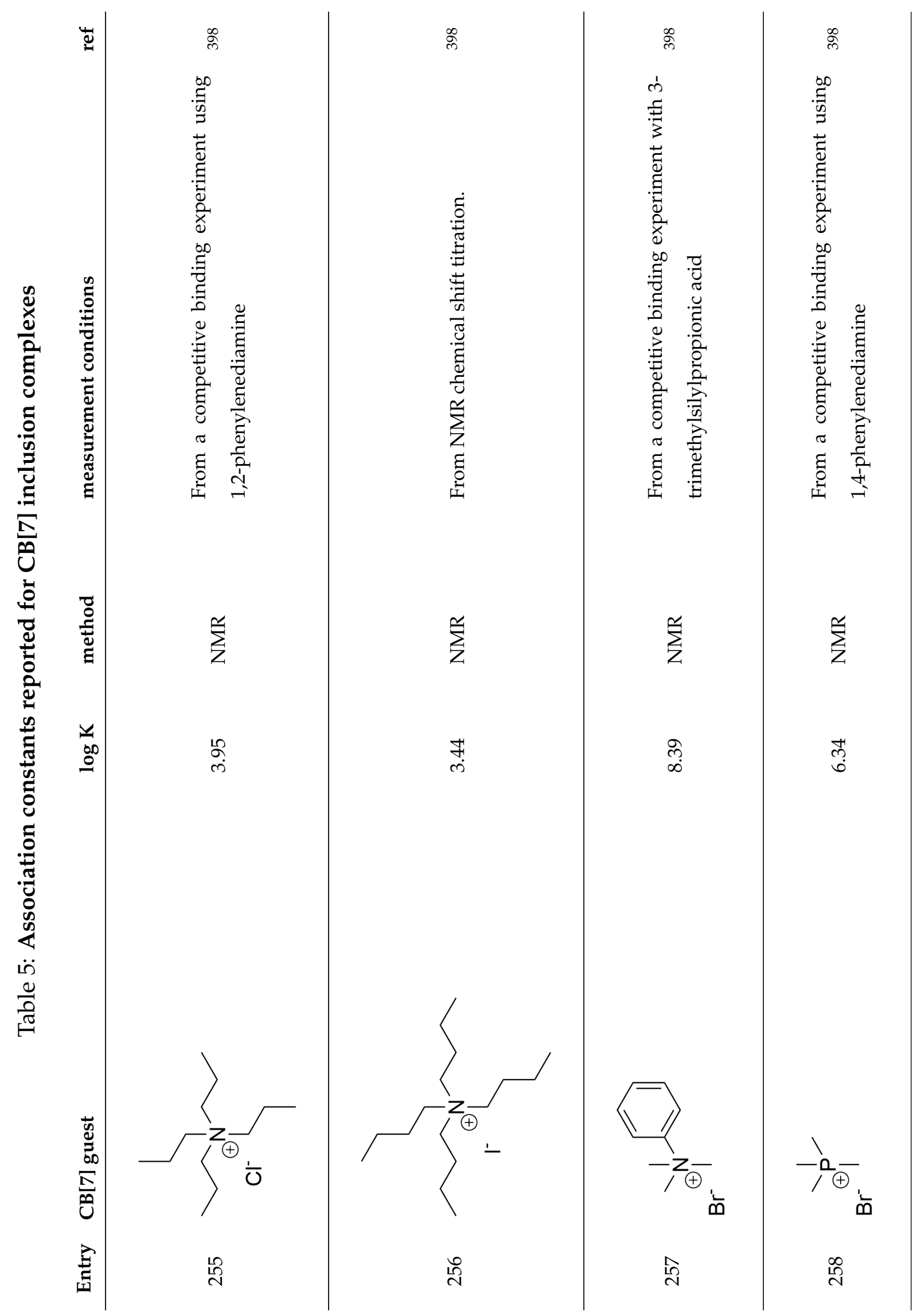

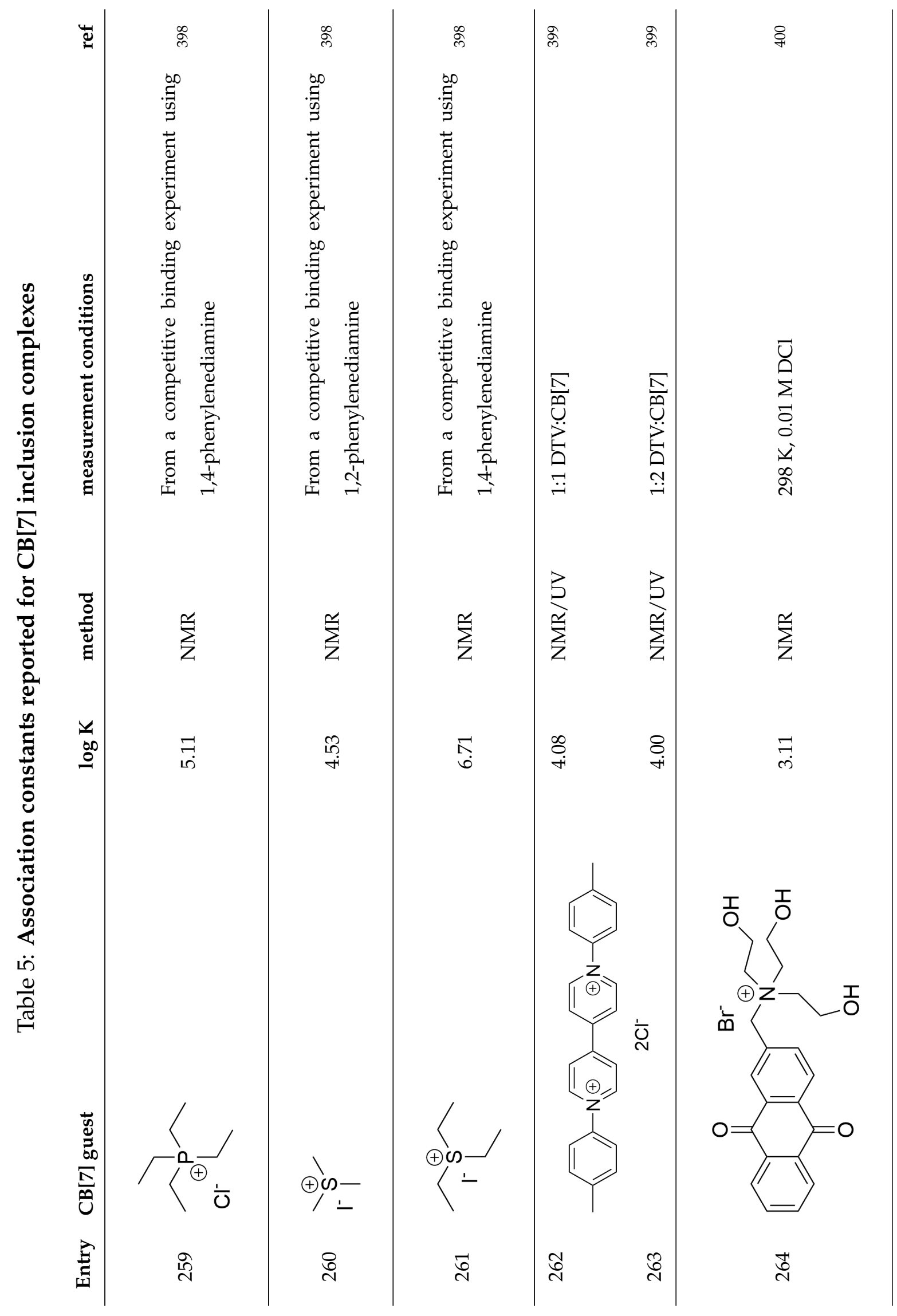

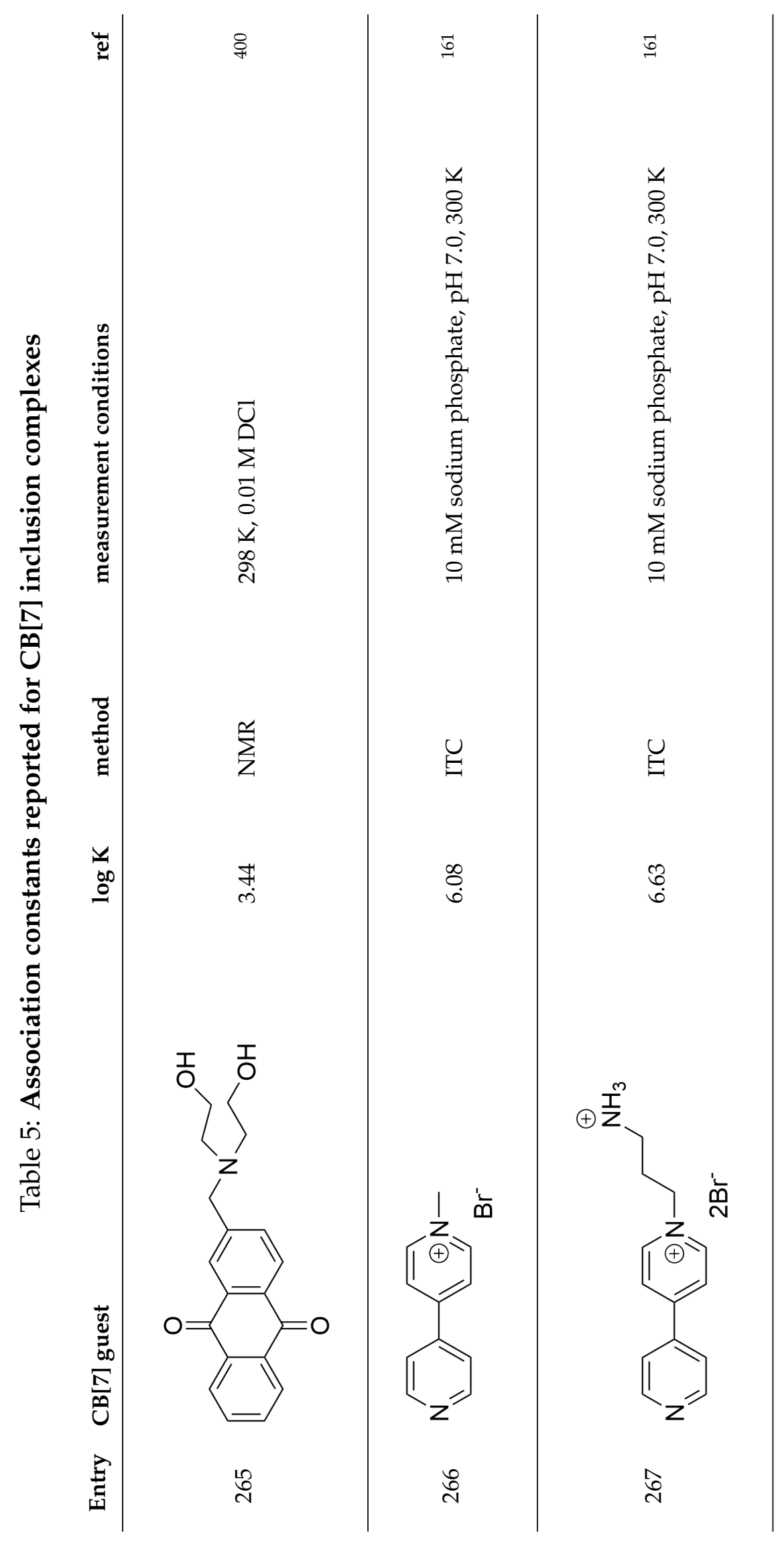

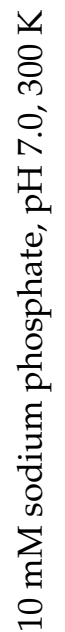

U

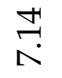

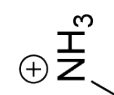

$\oplus \frac{m}{I^{\prime}}$

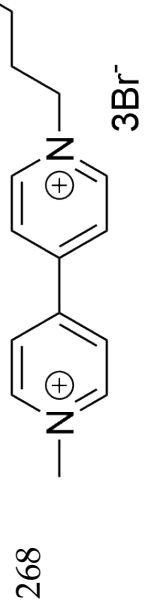



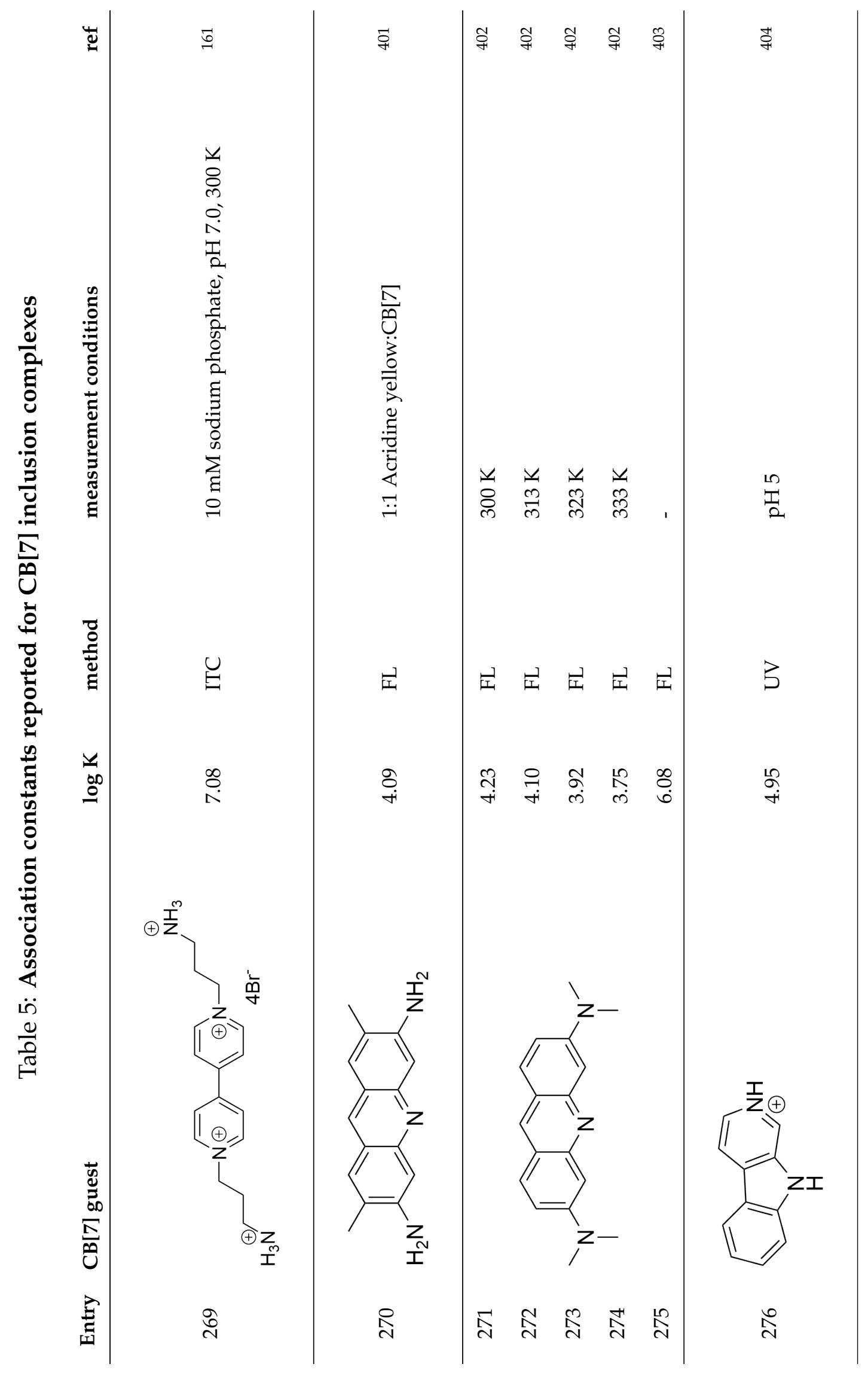

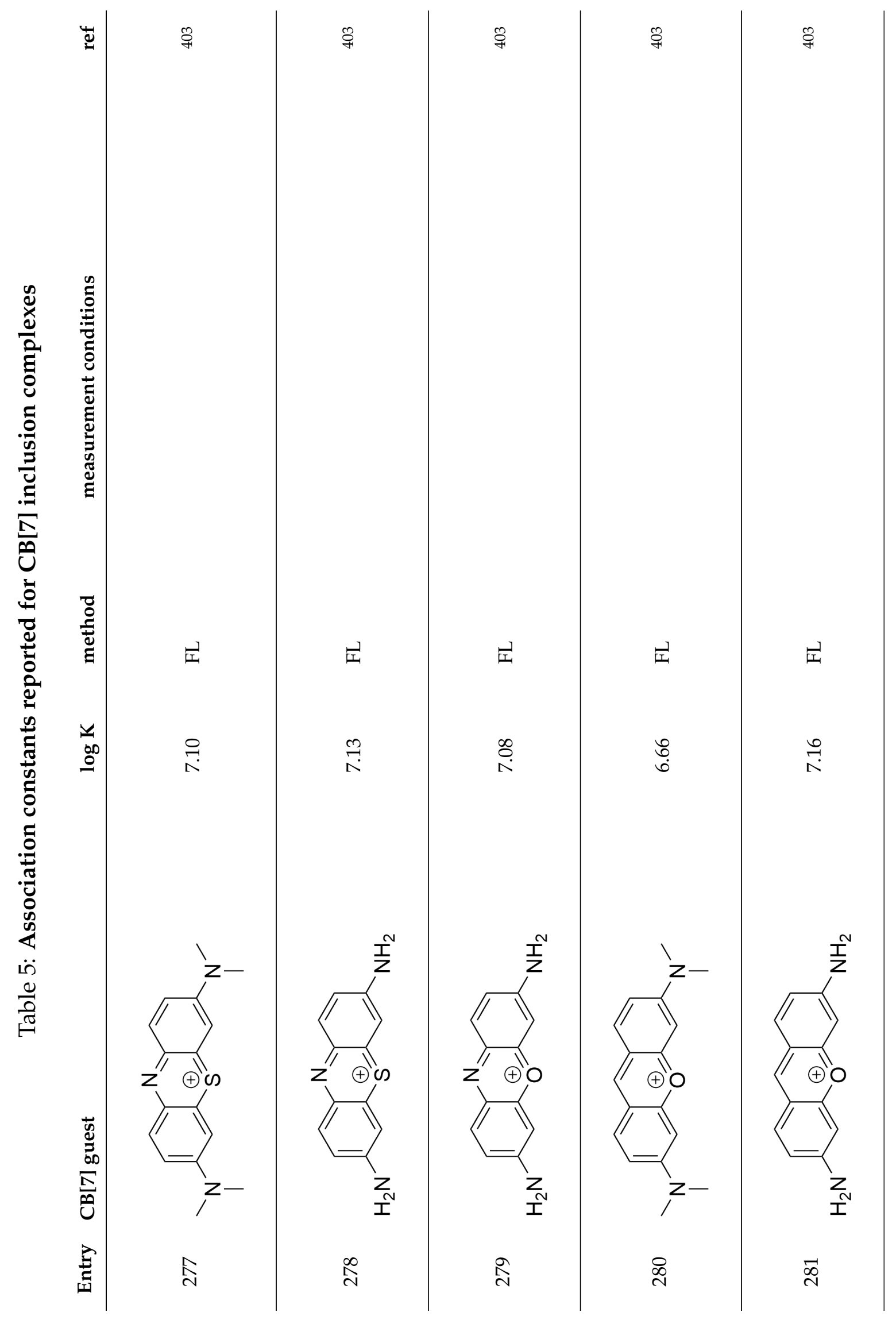

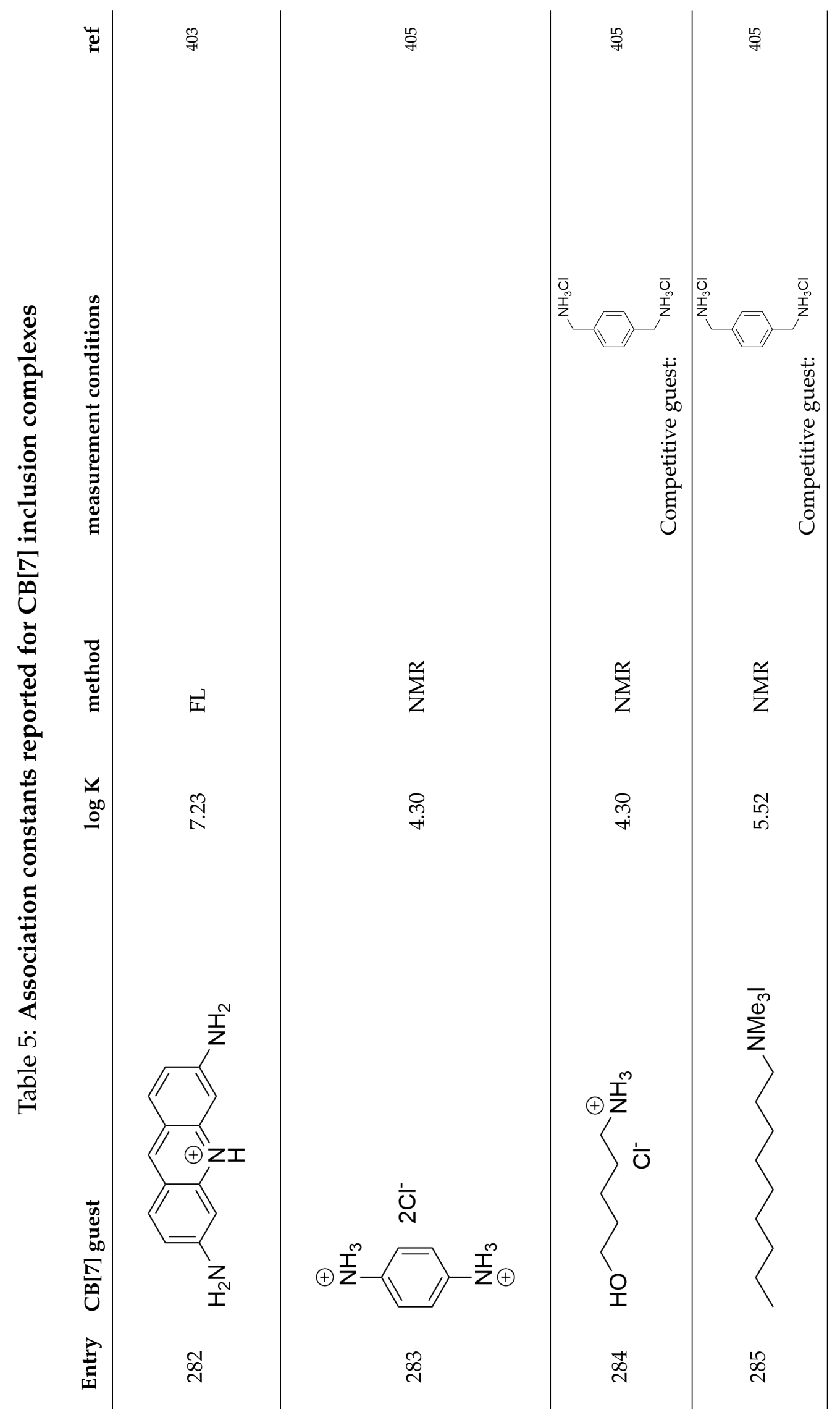

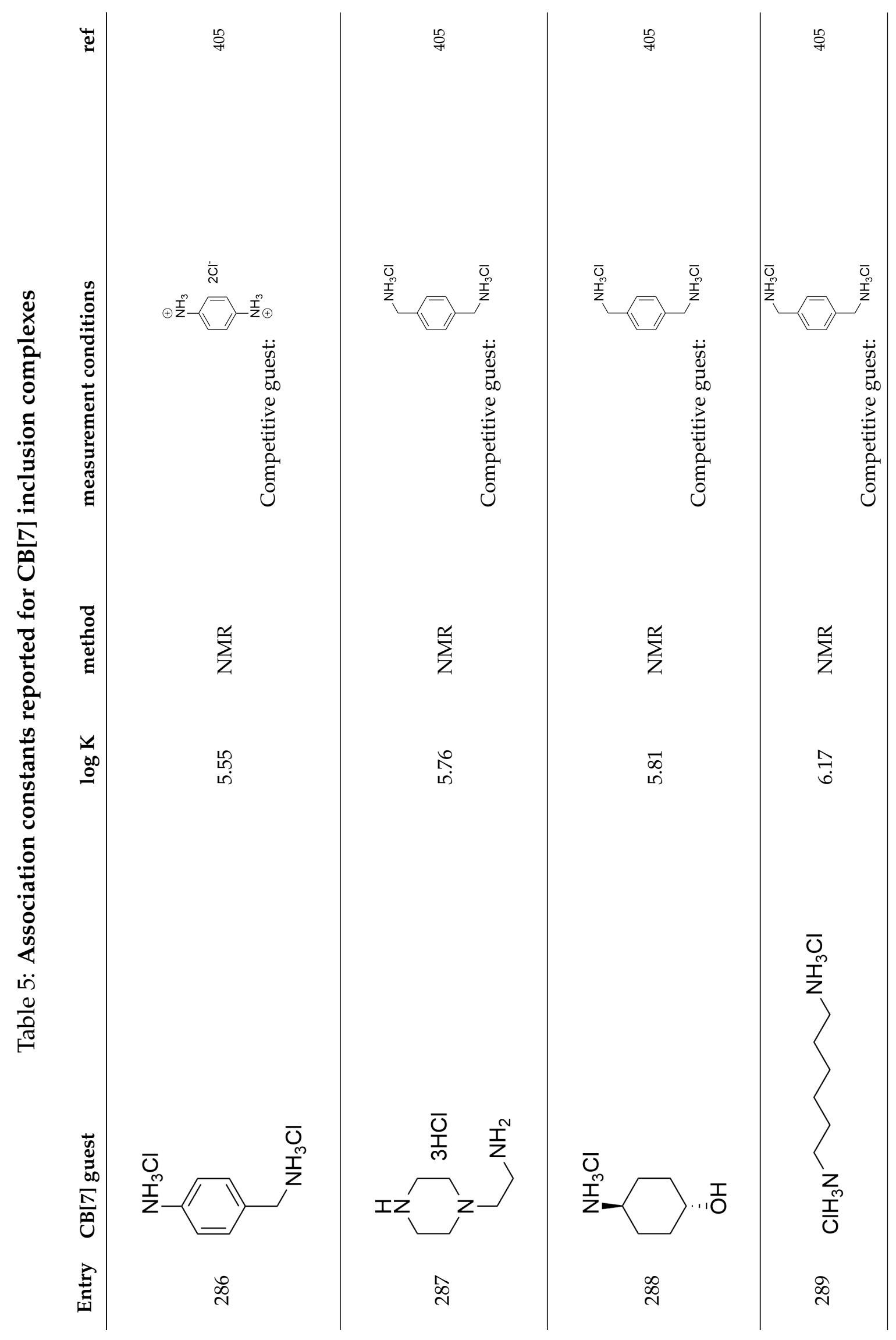


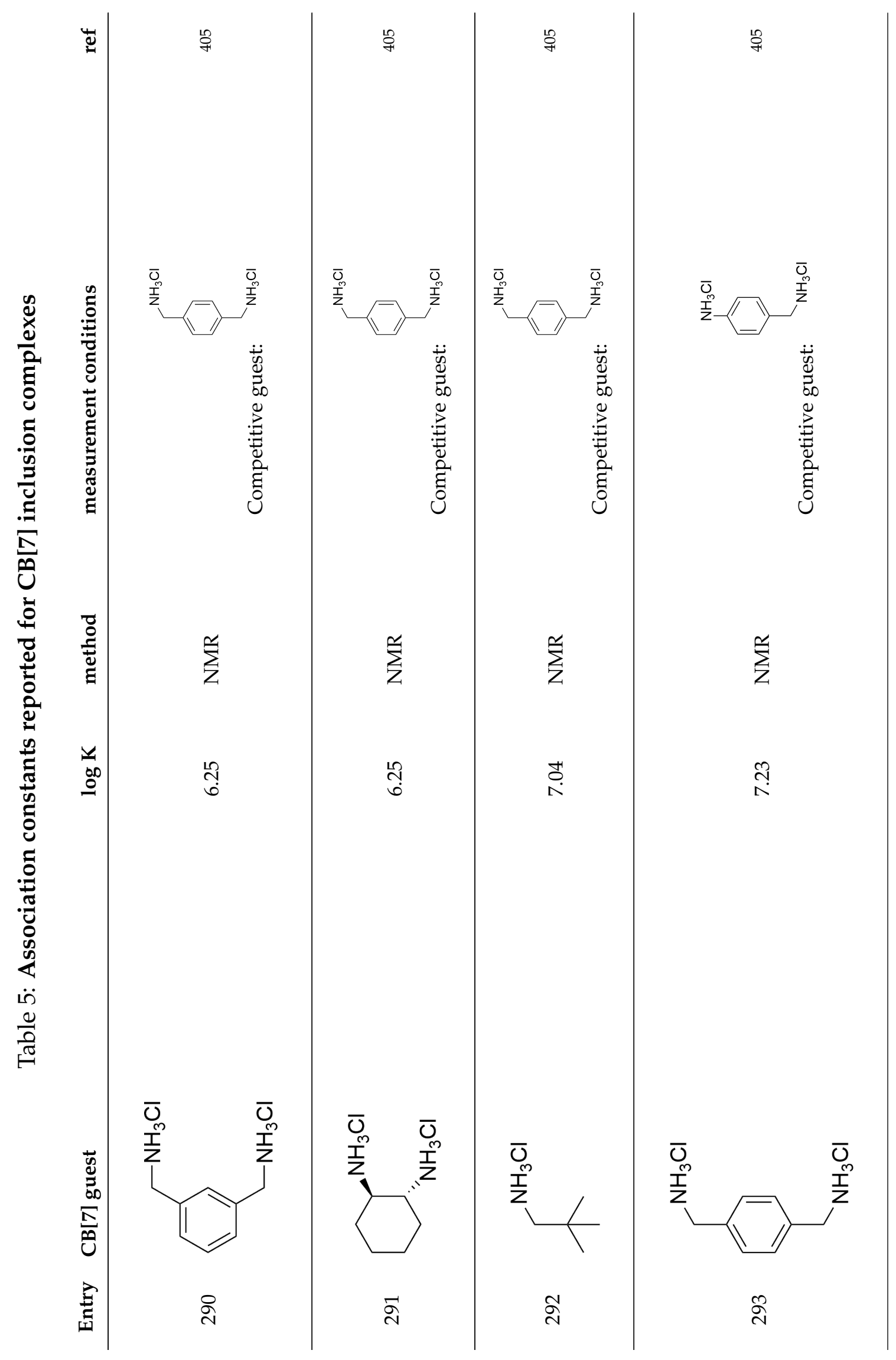




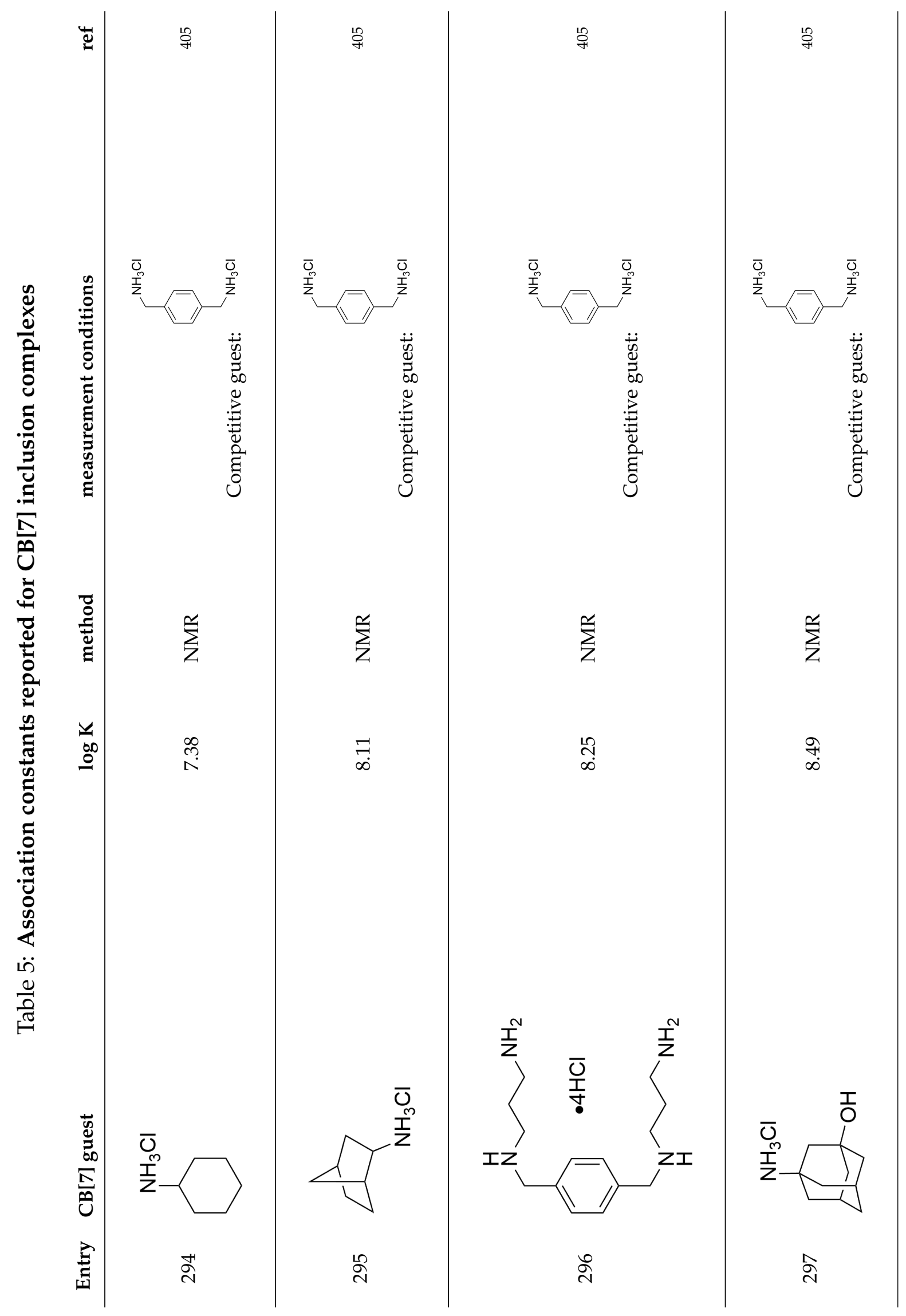



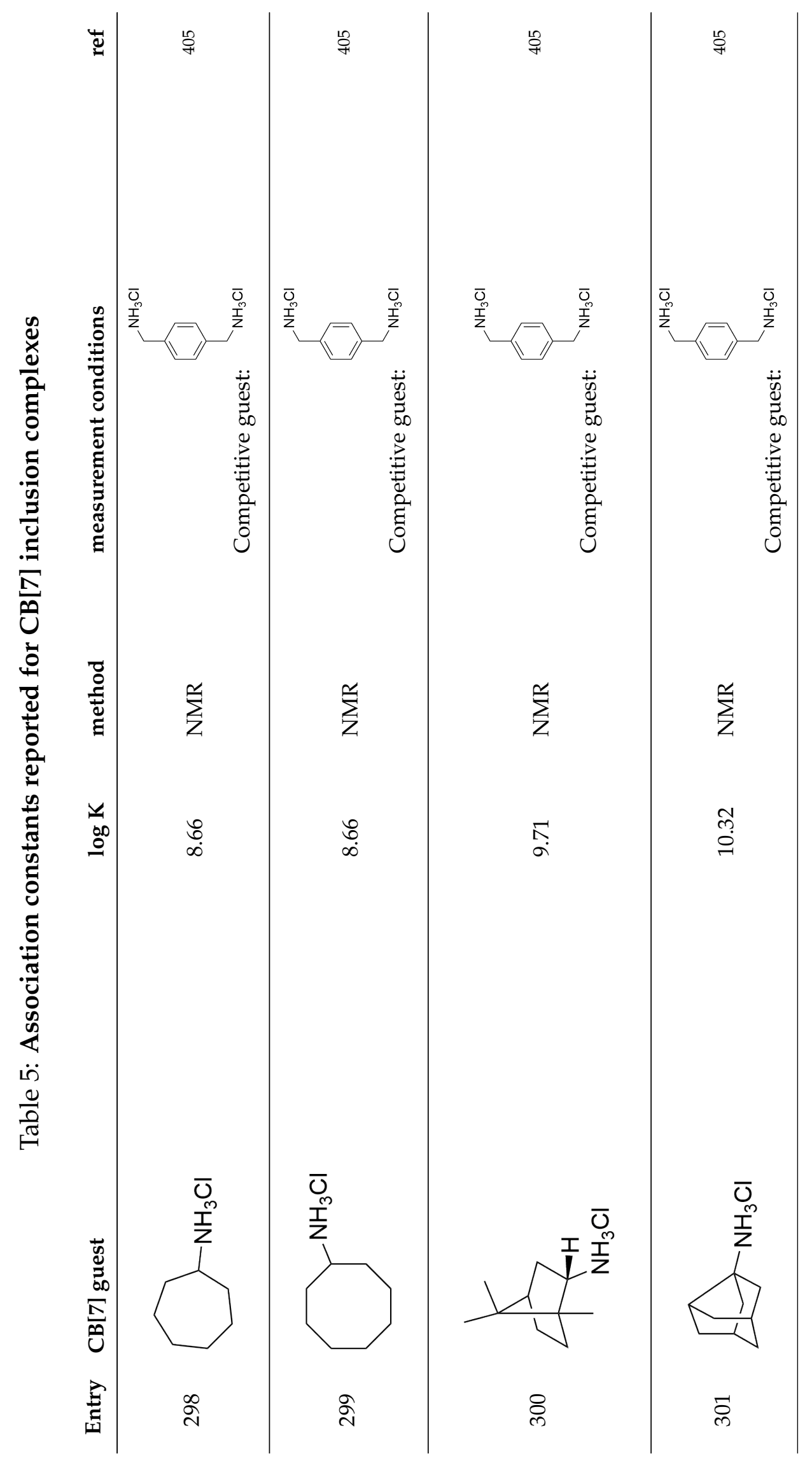

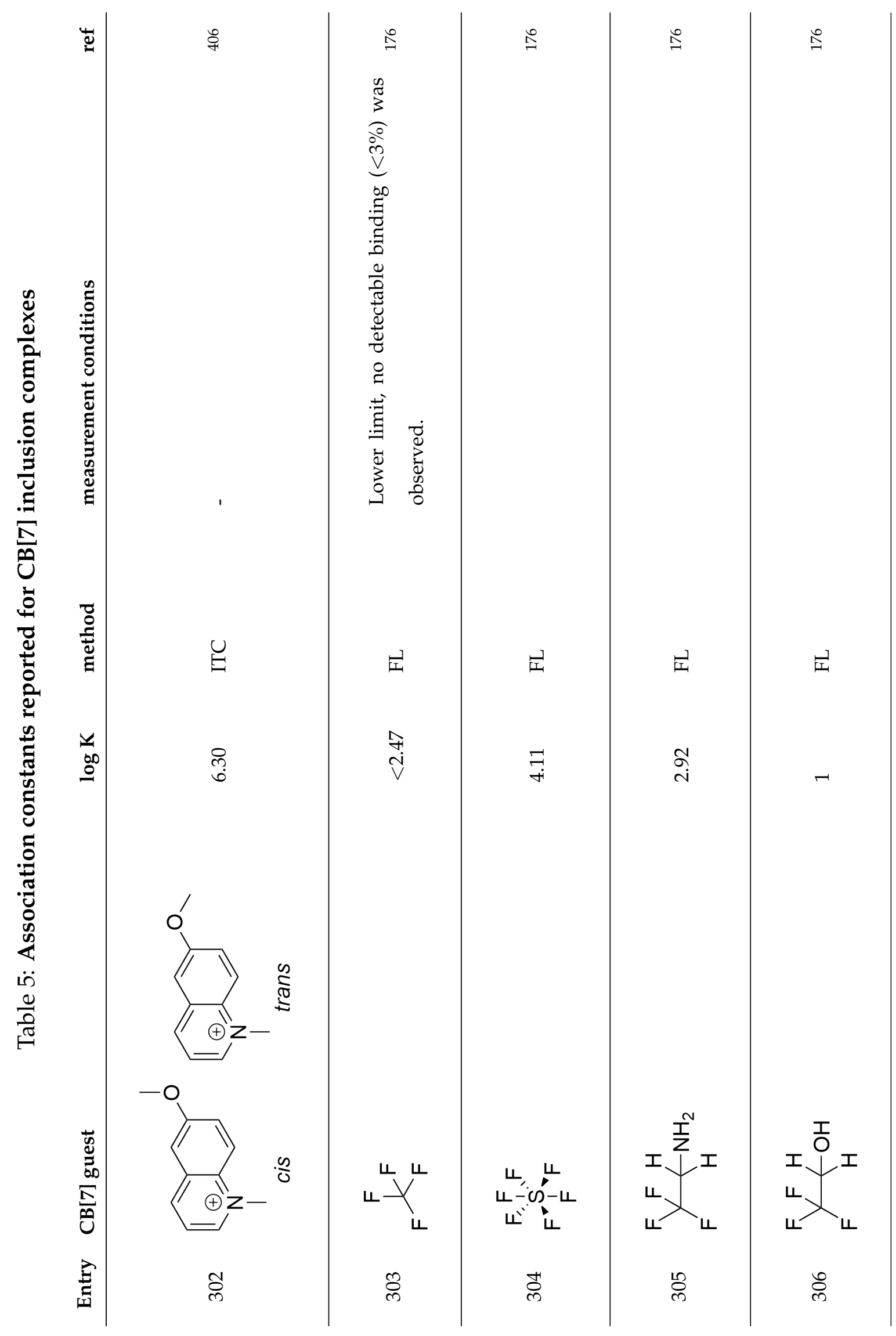

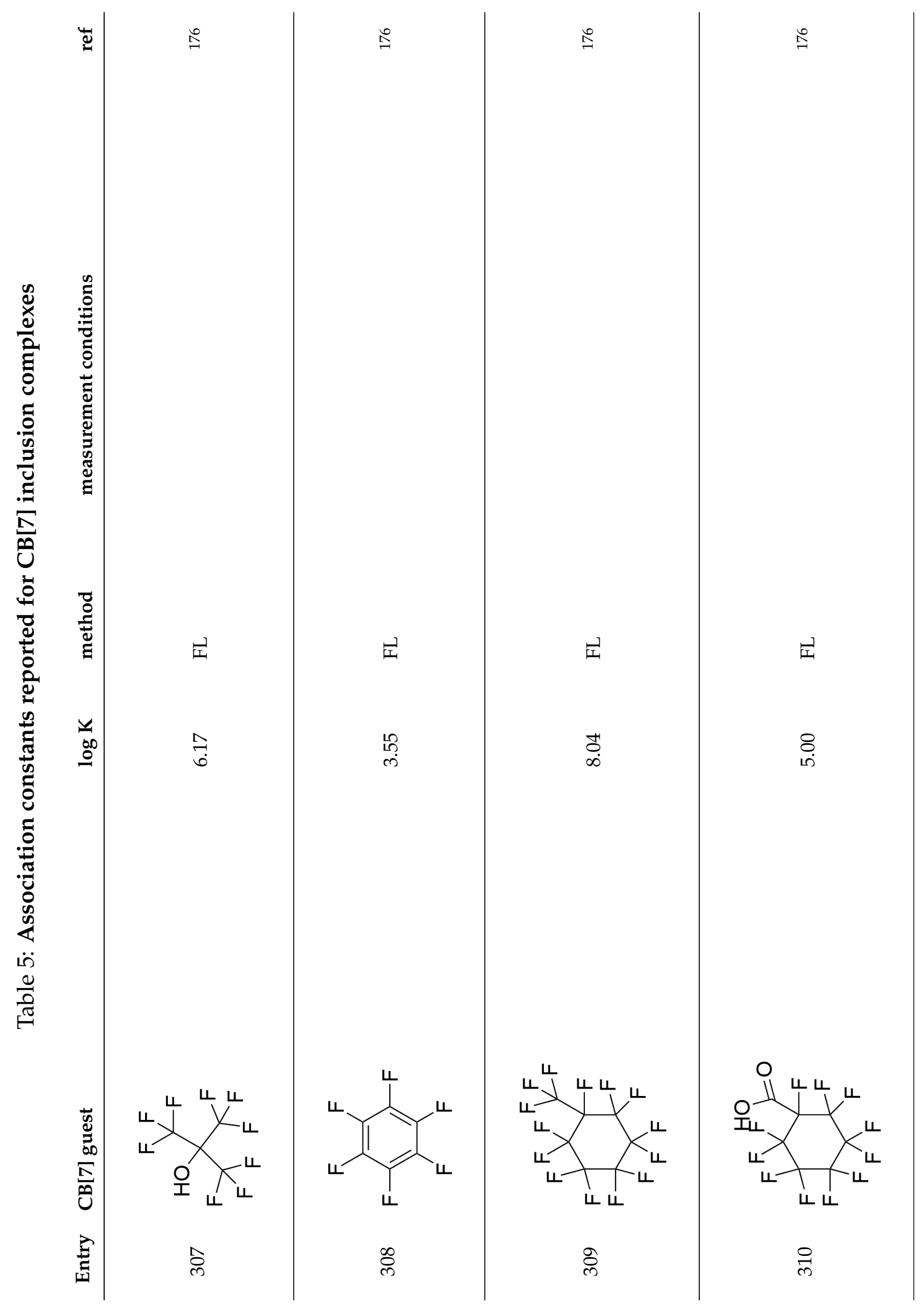

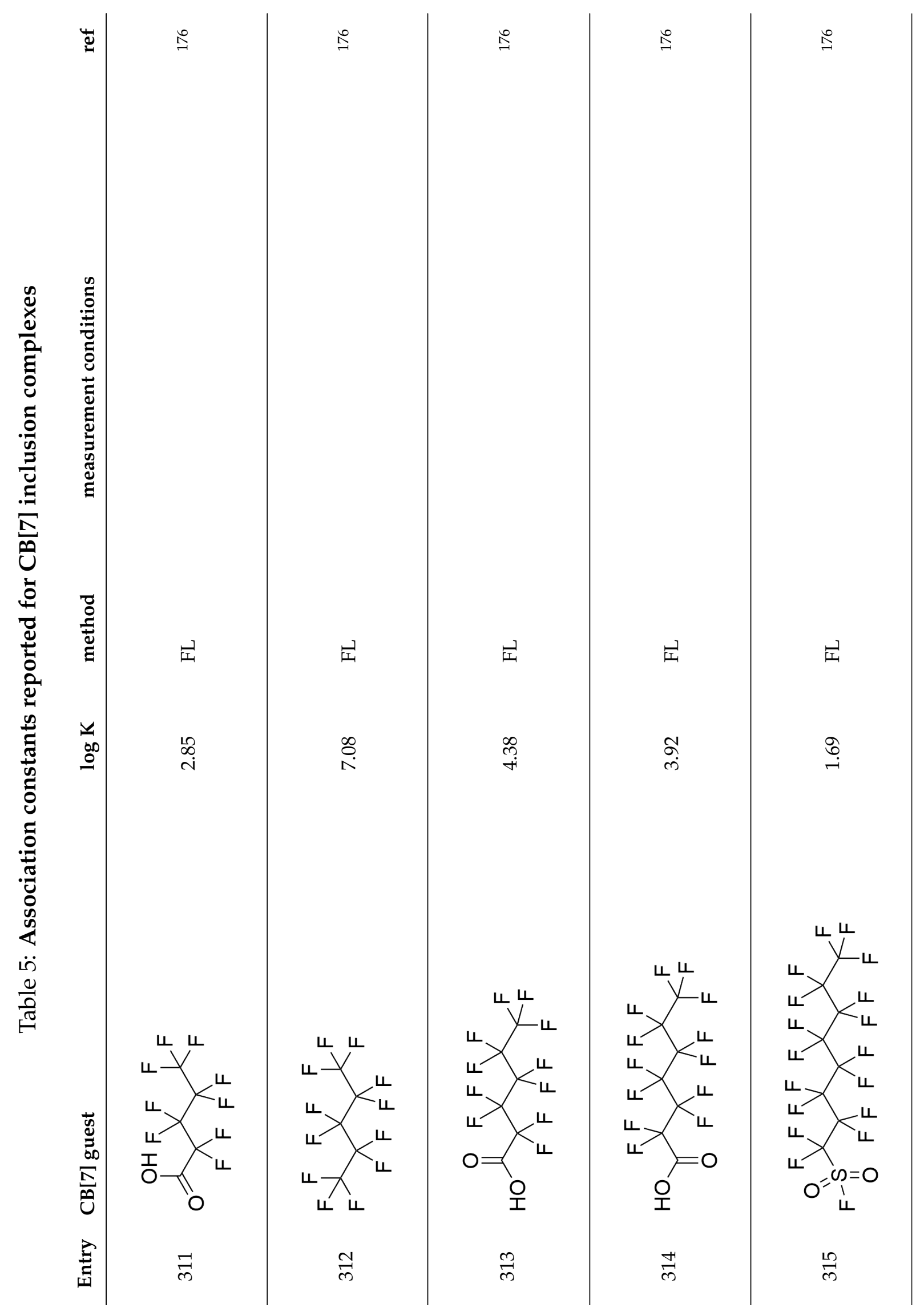


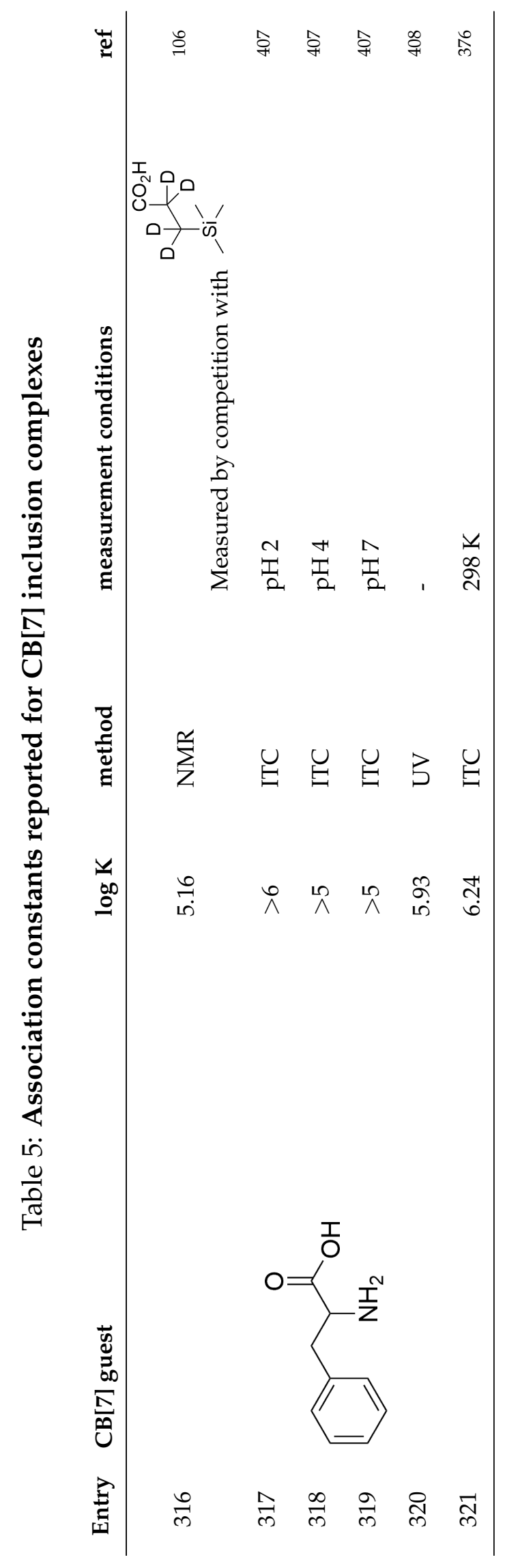




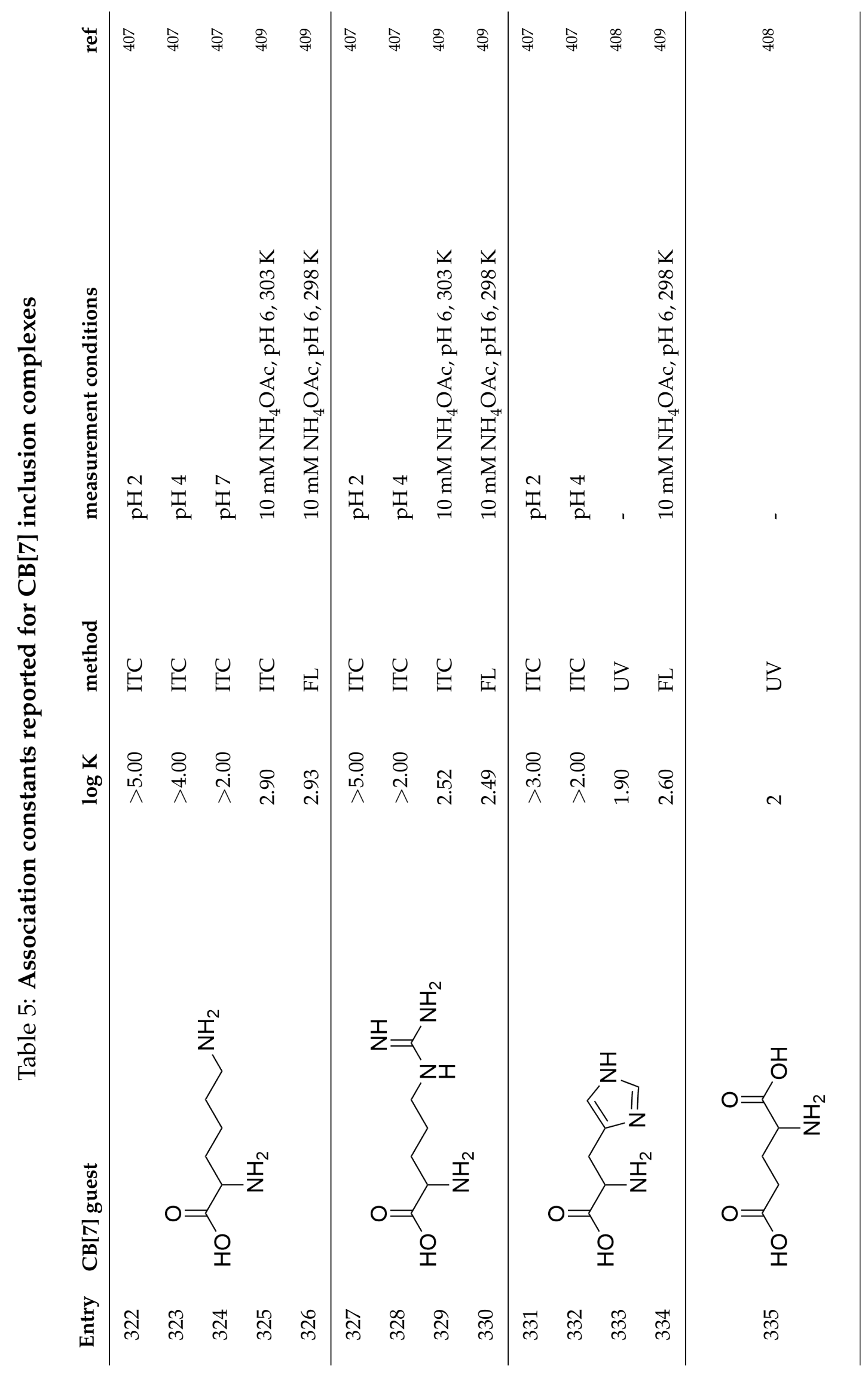



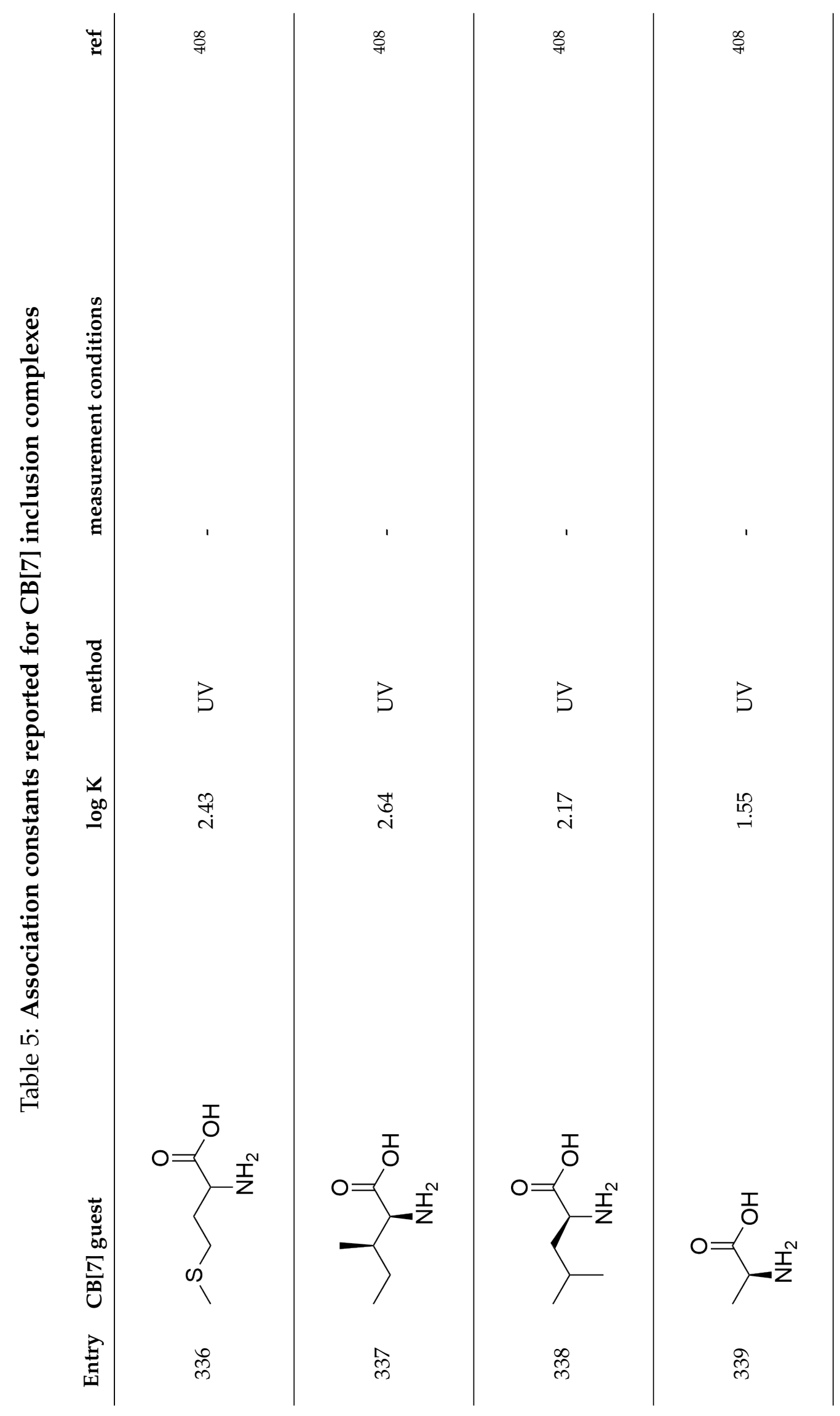


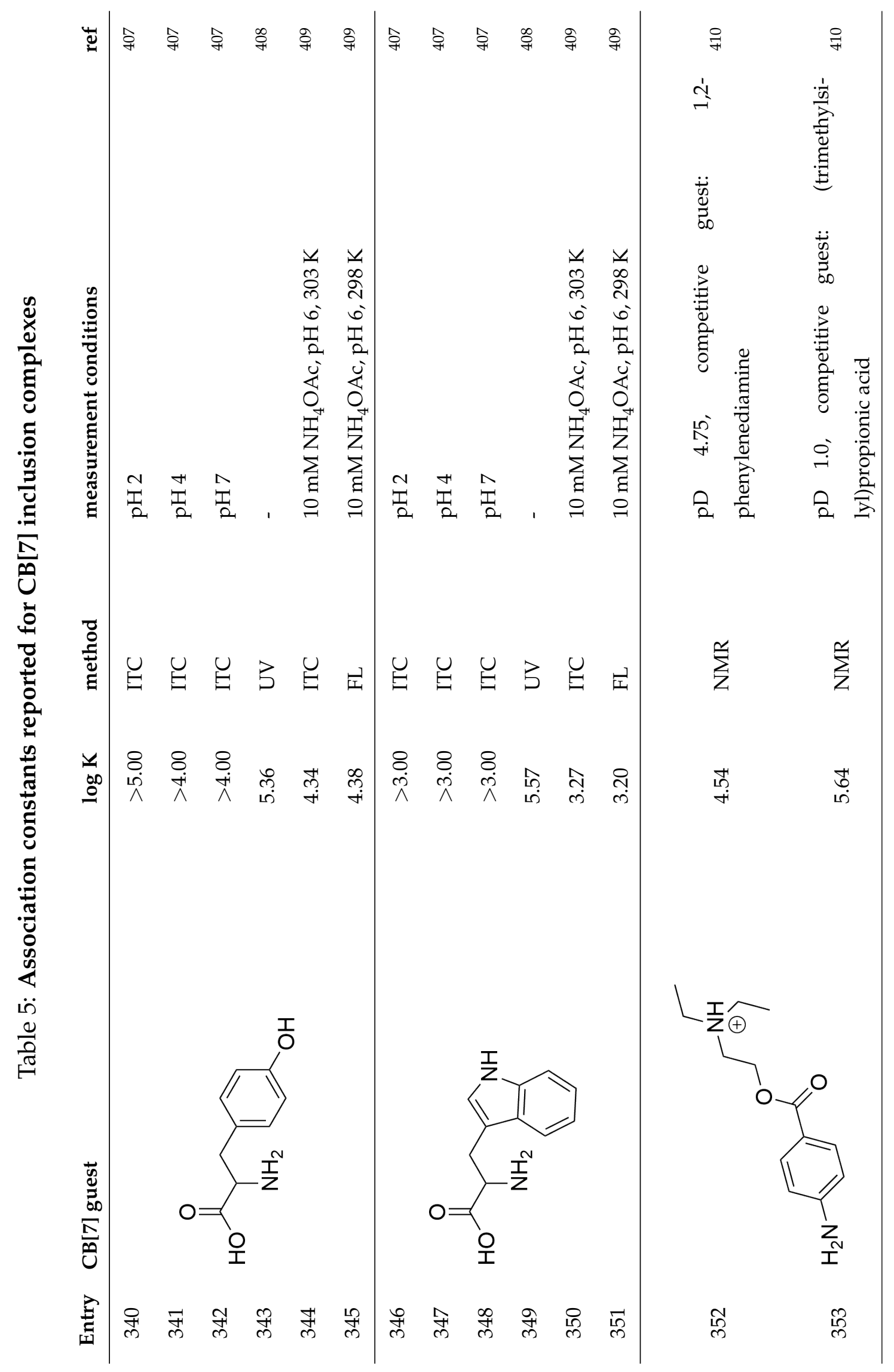




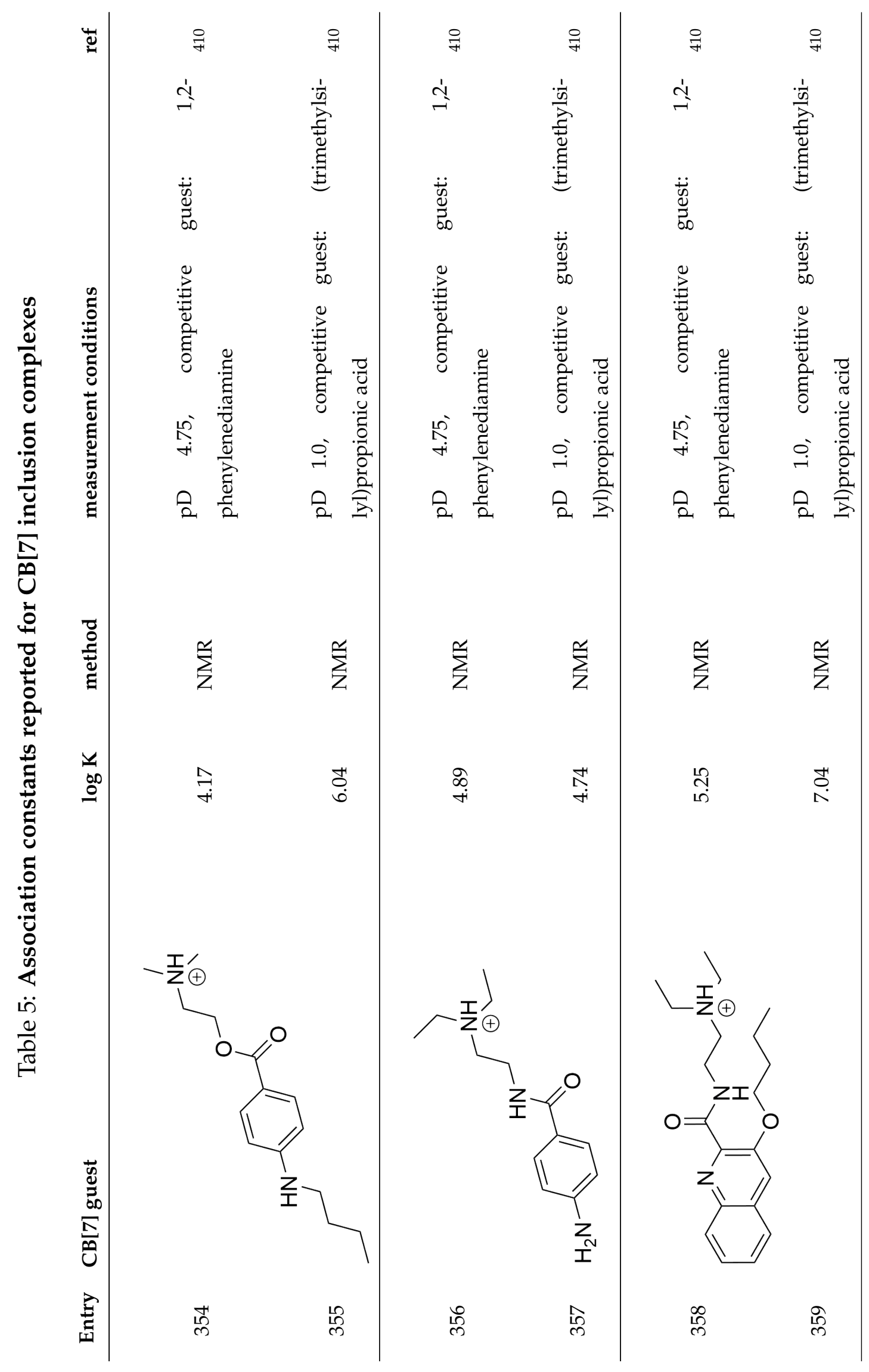




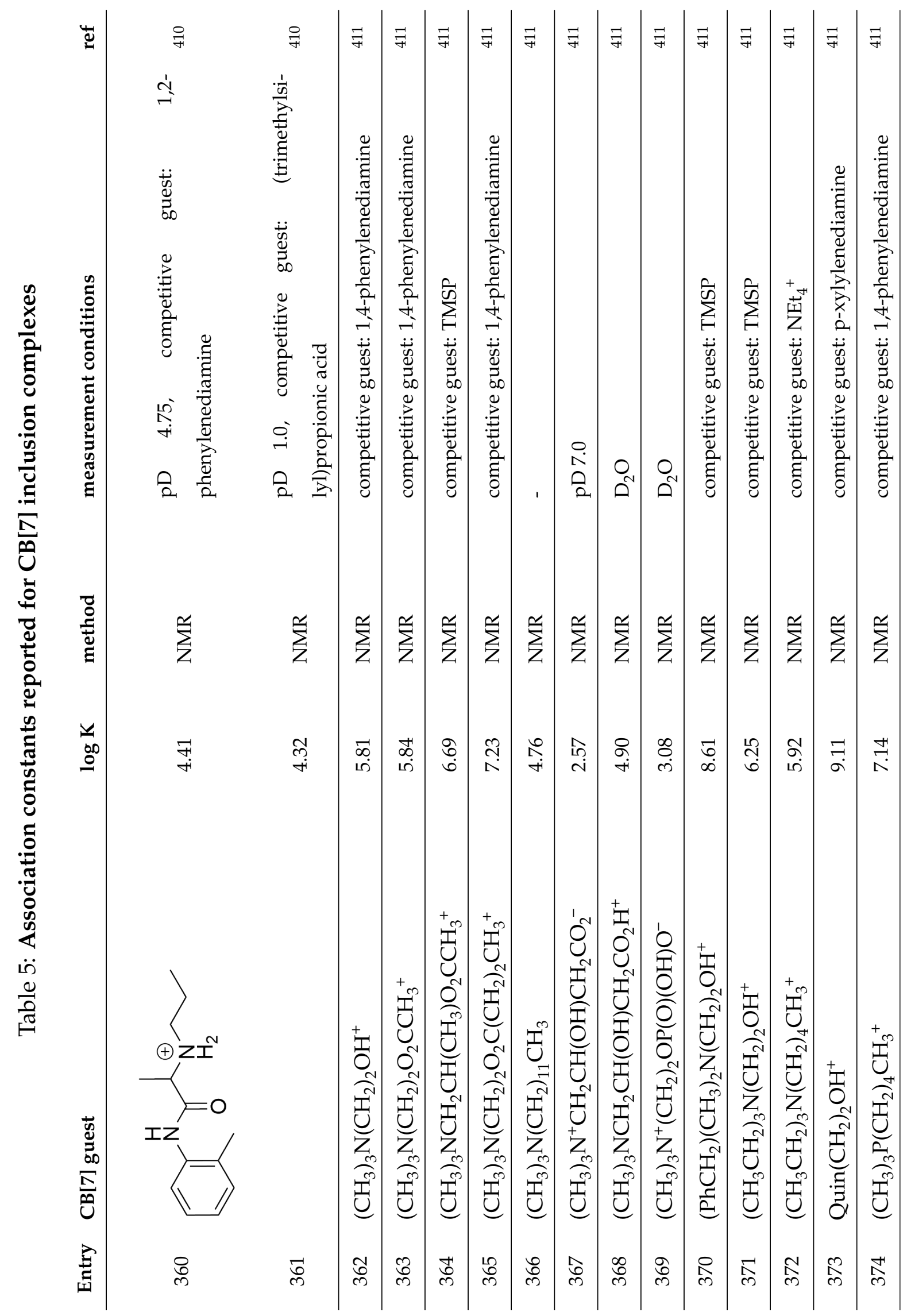



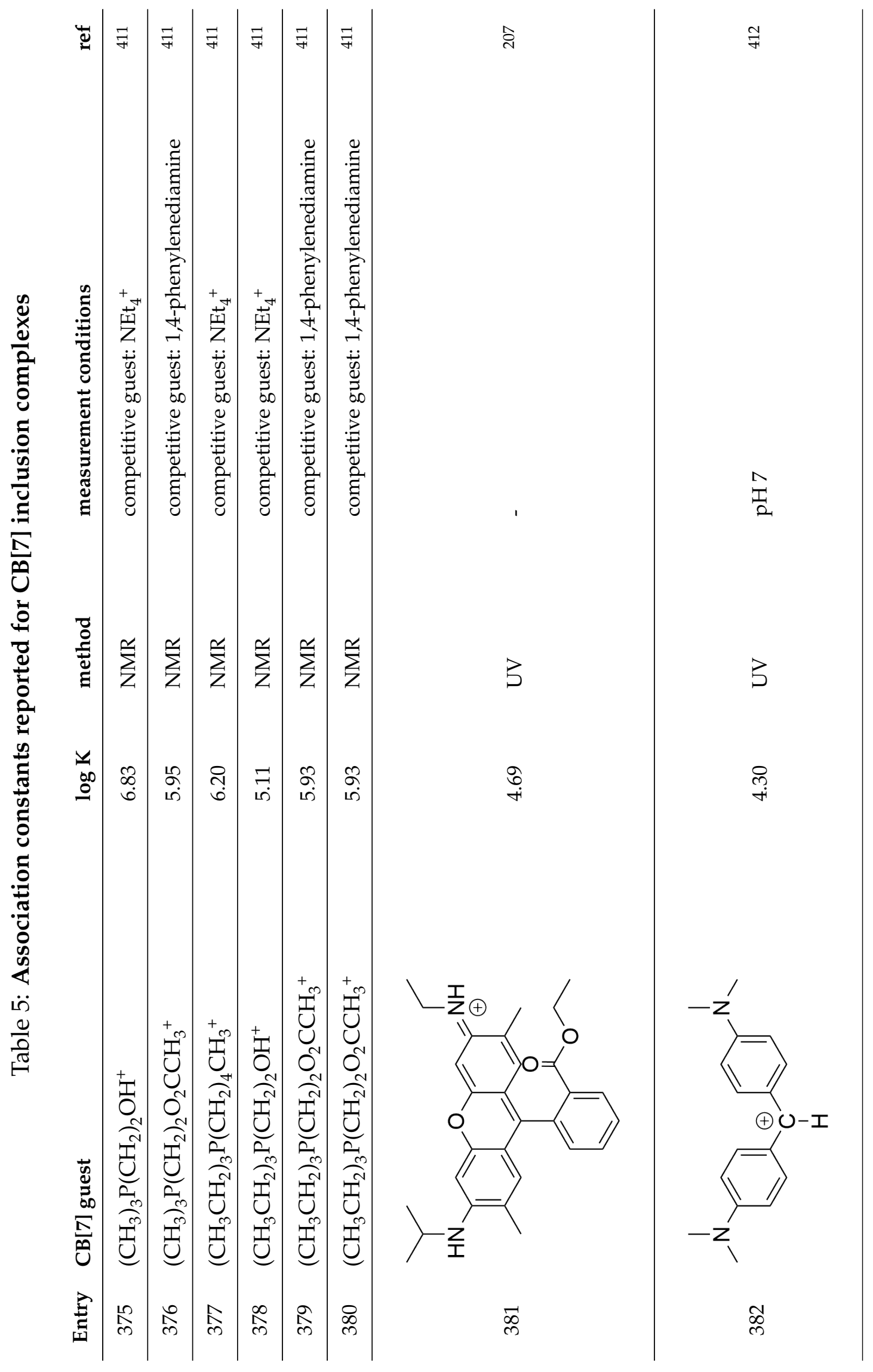

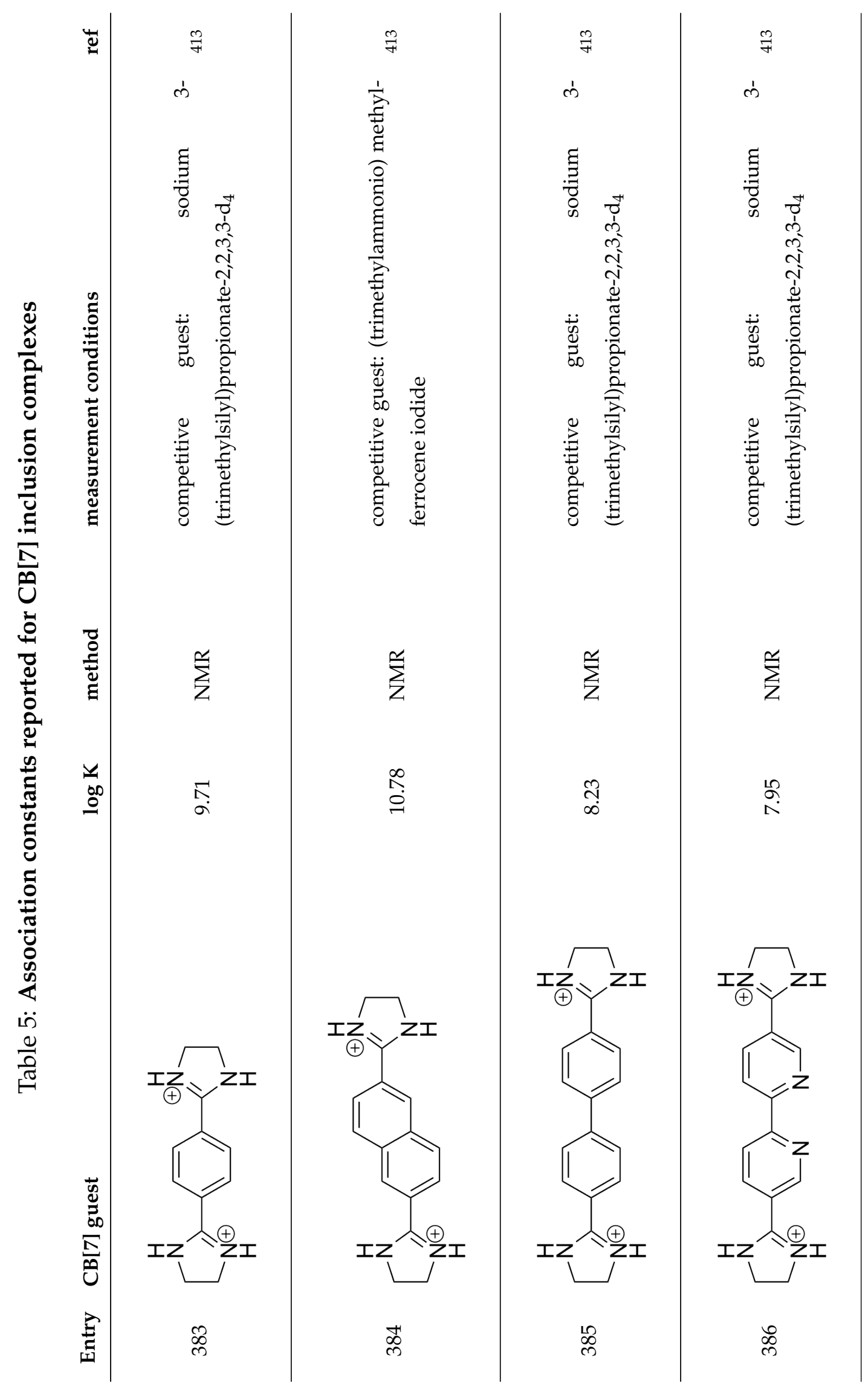


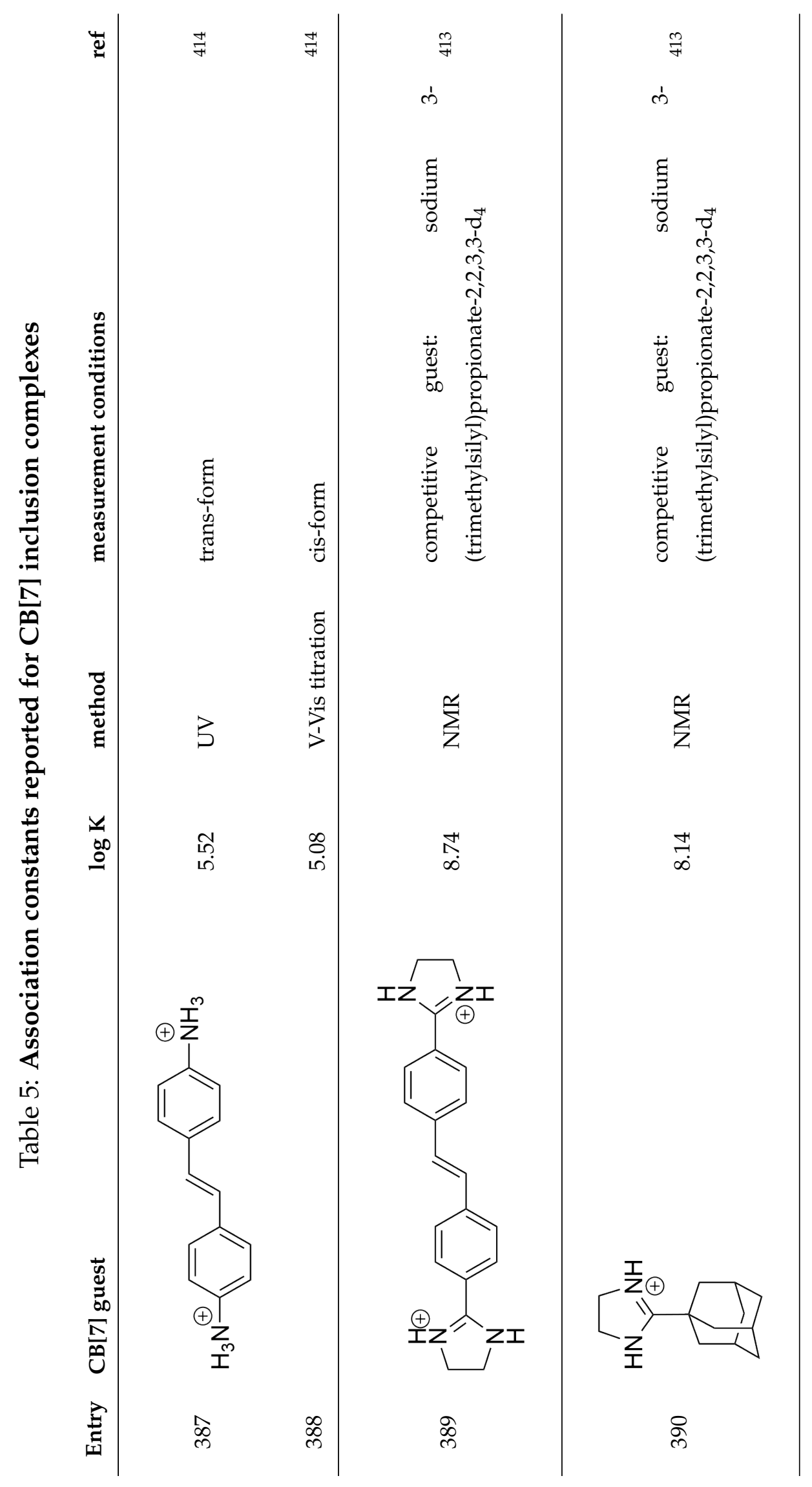




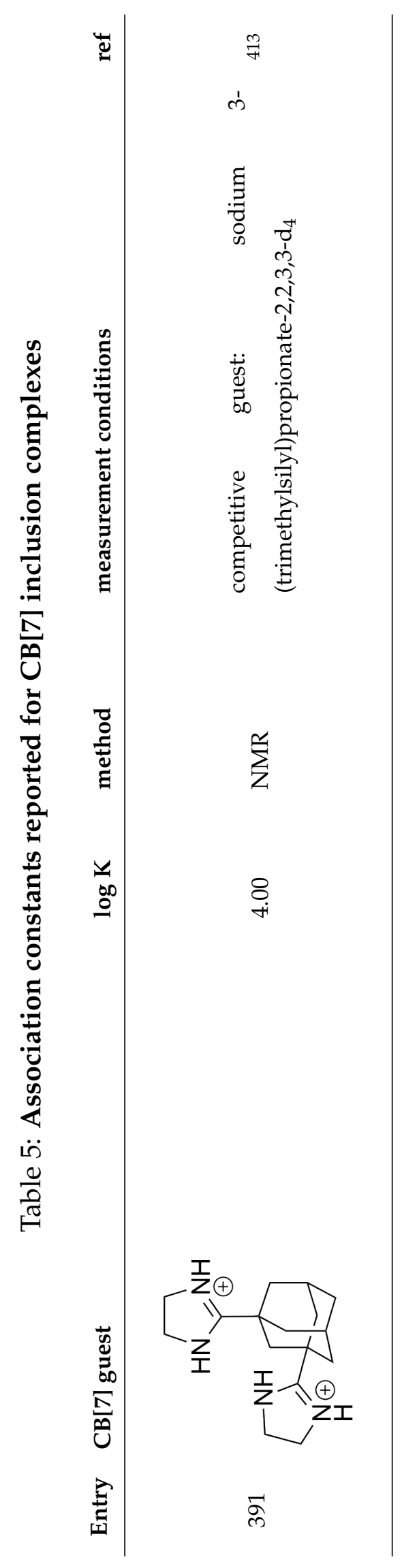




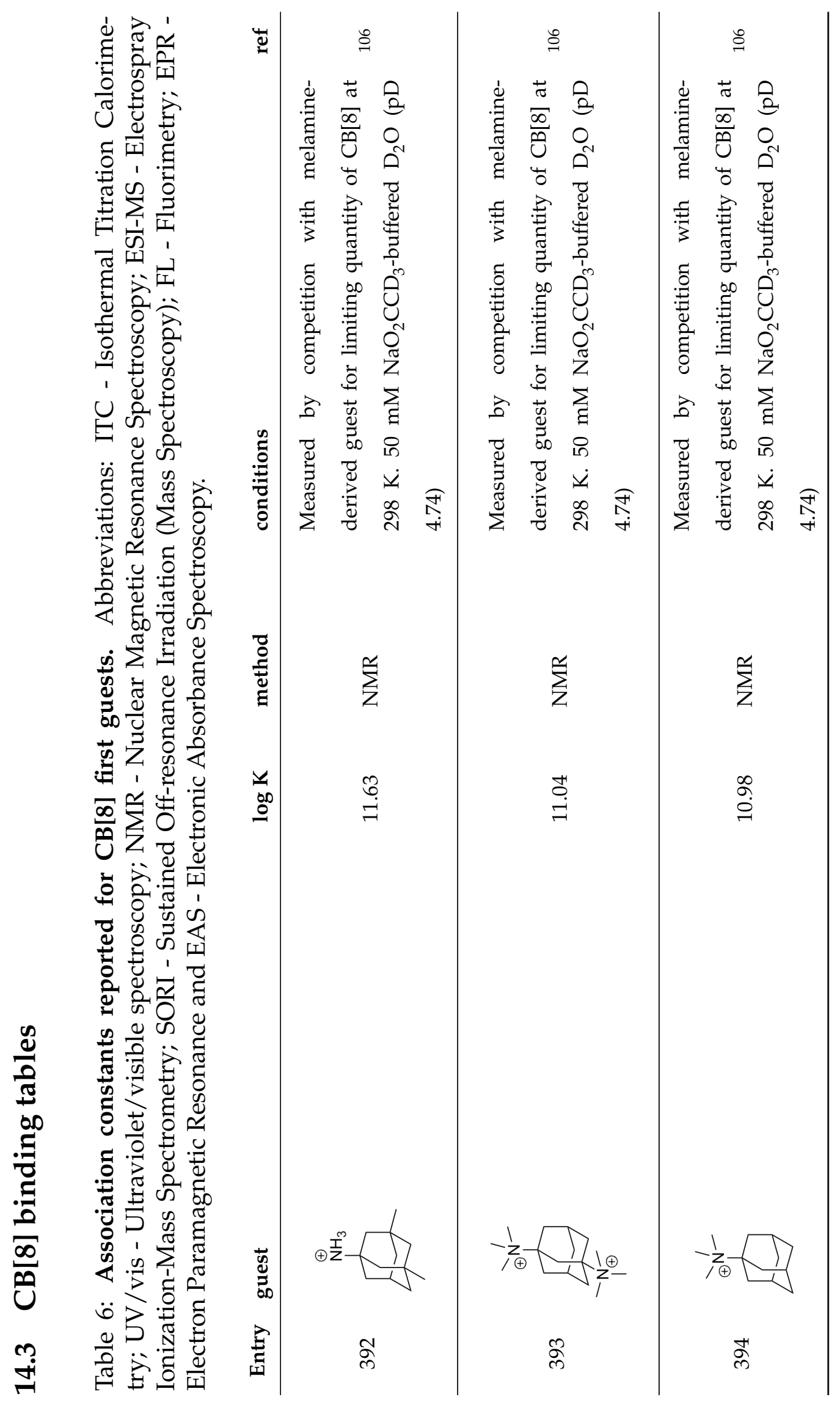




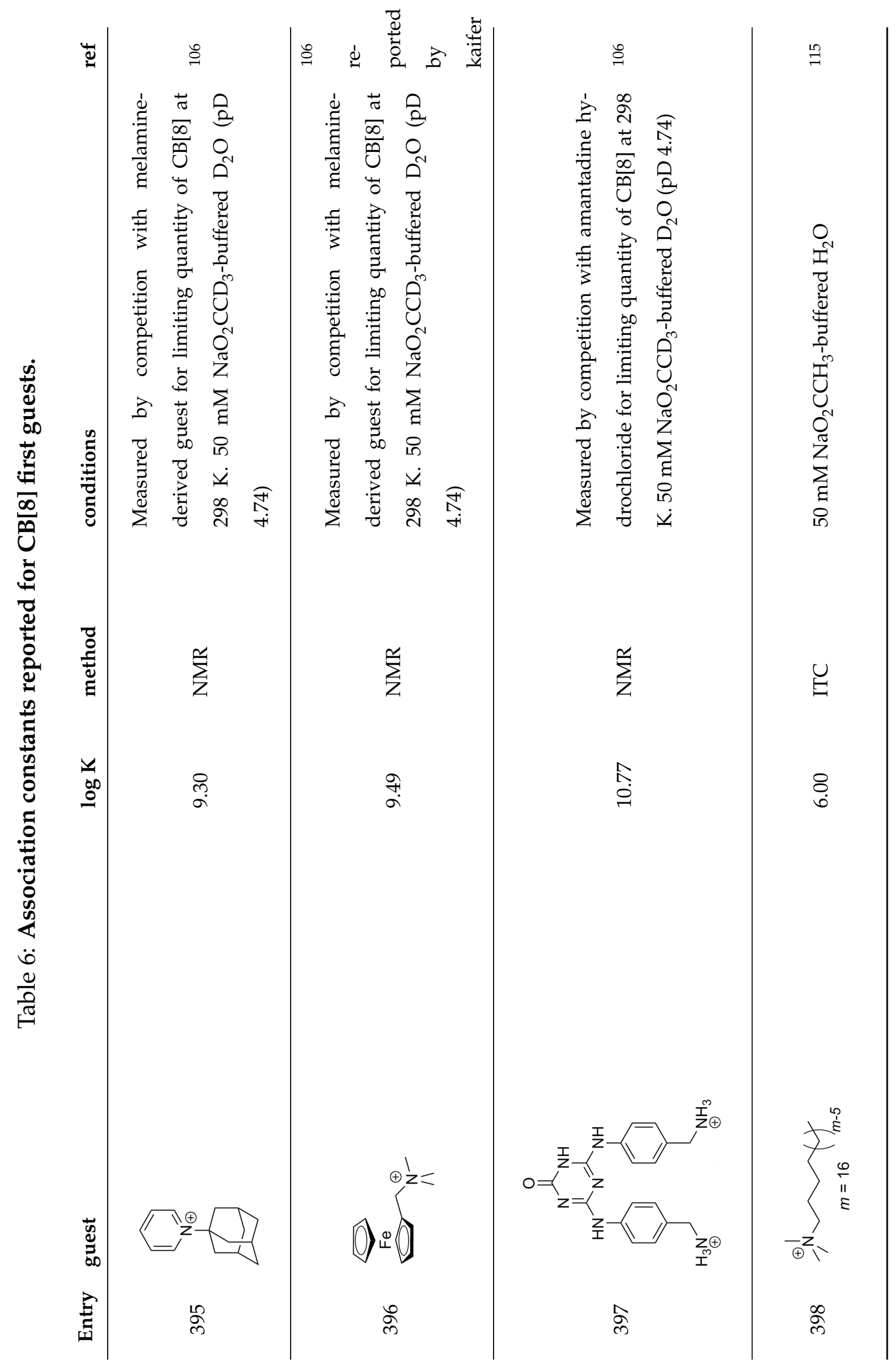




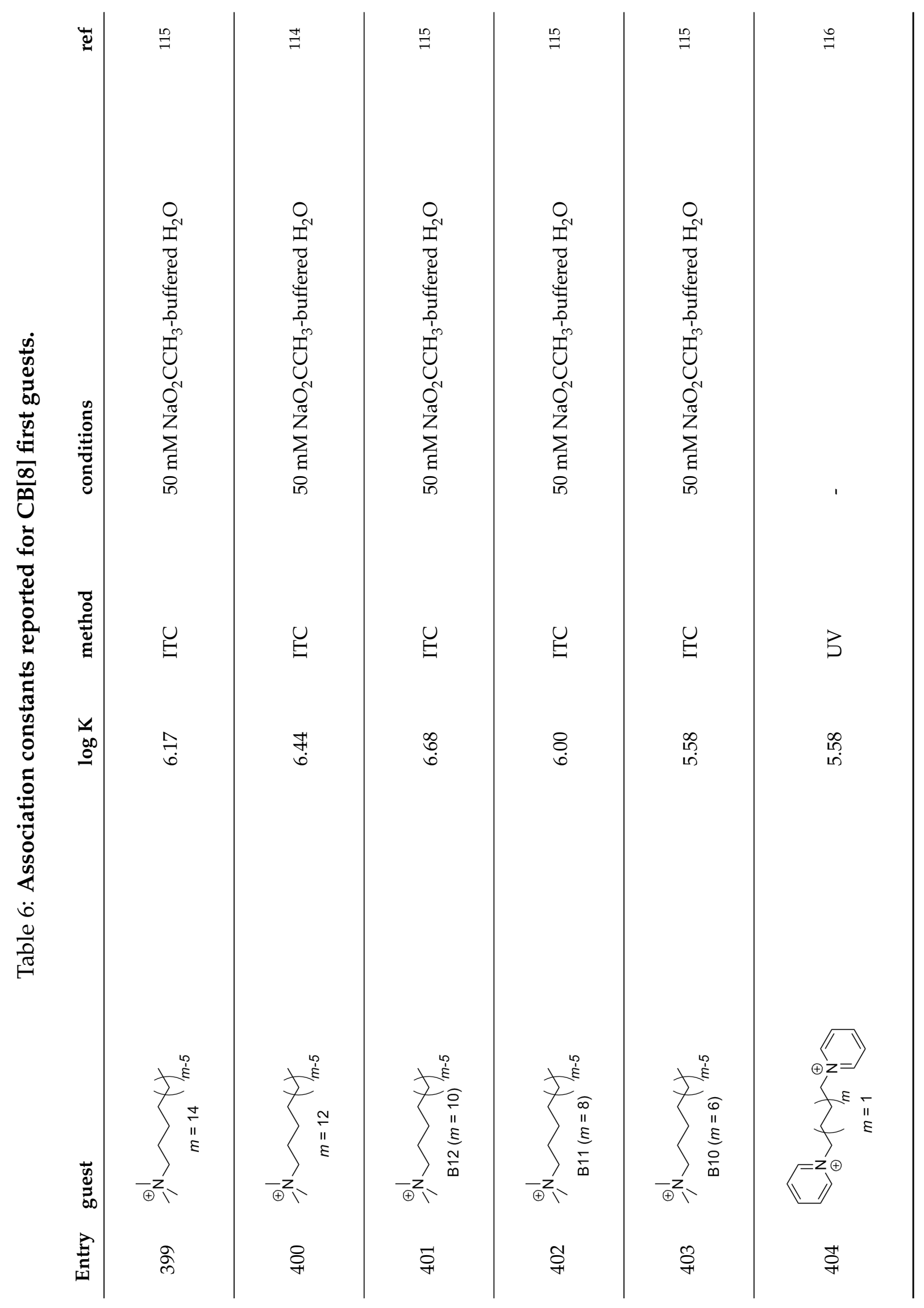




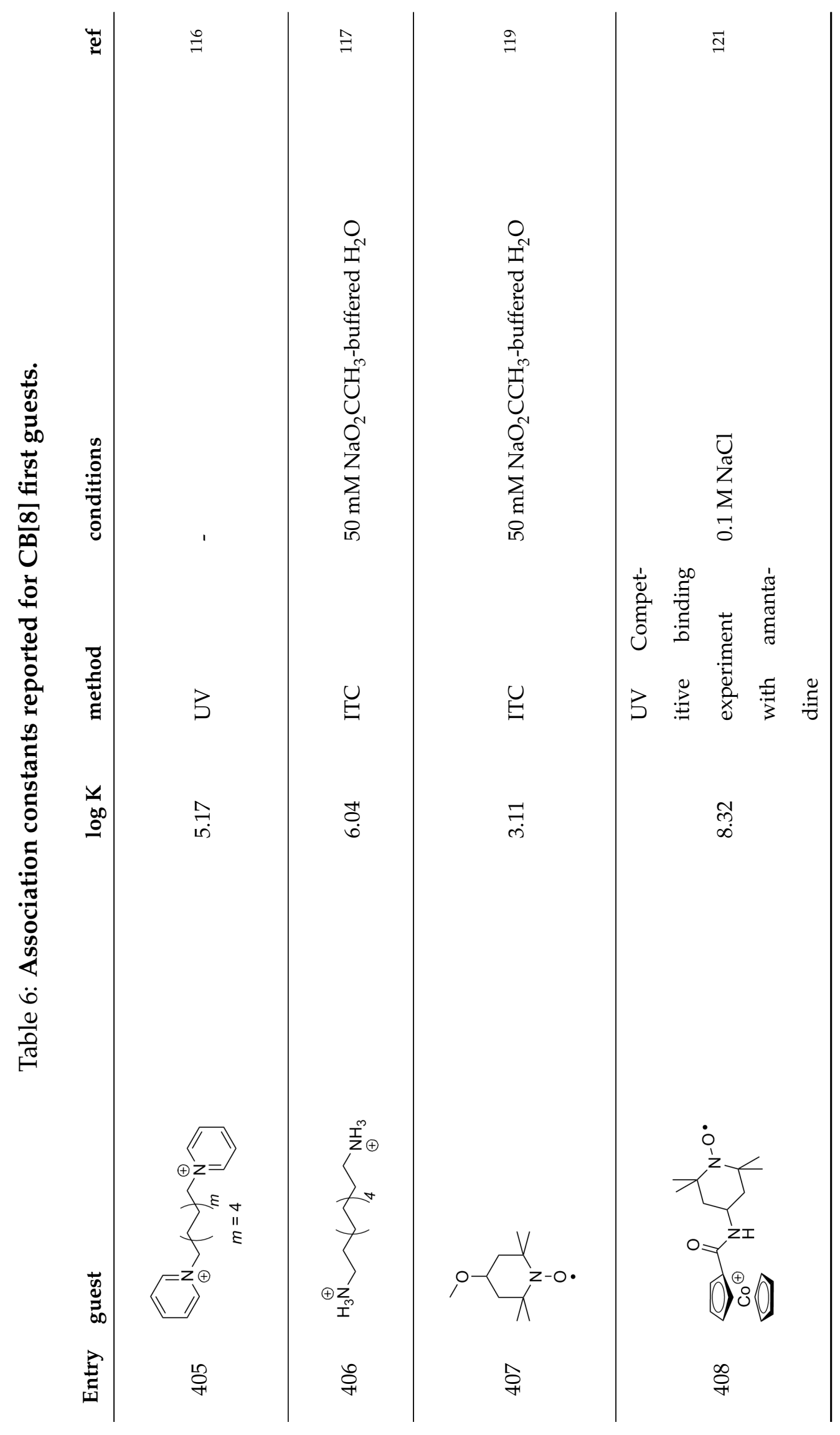




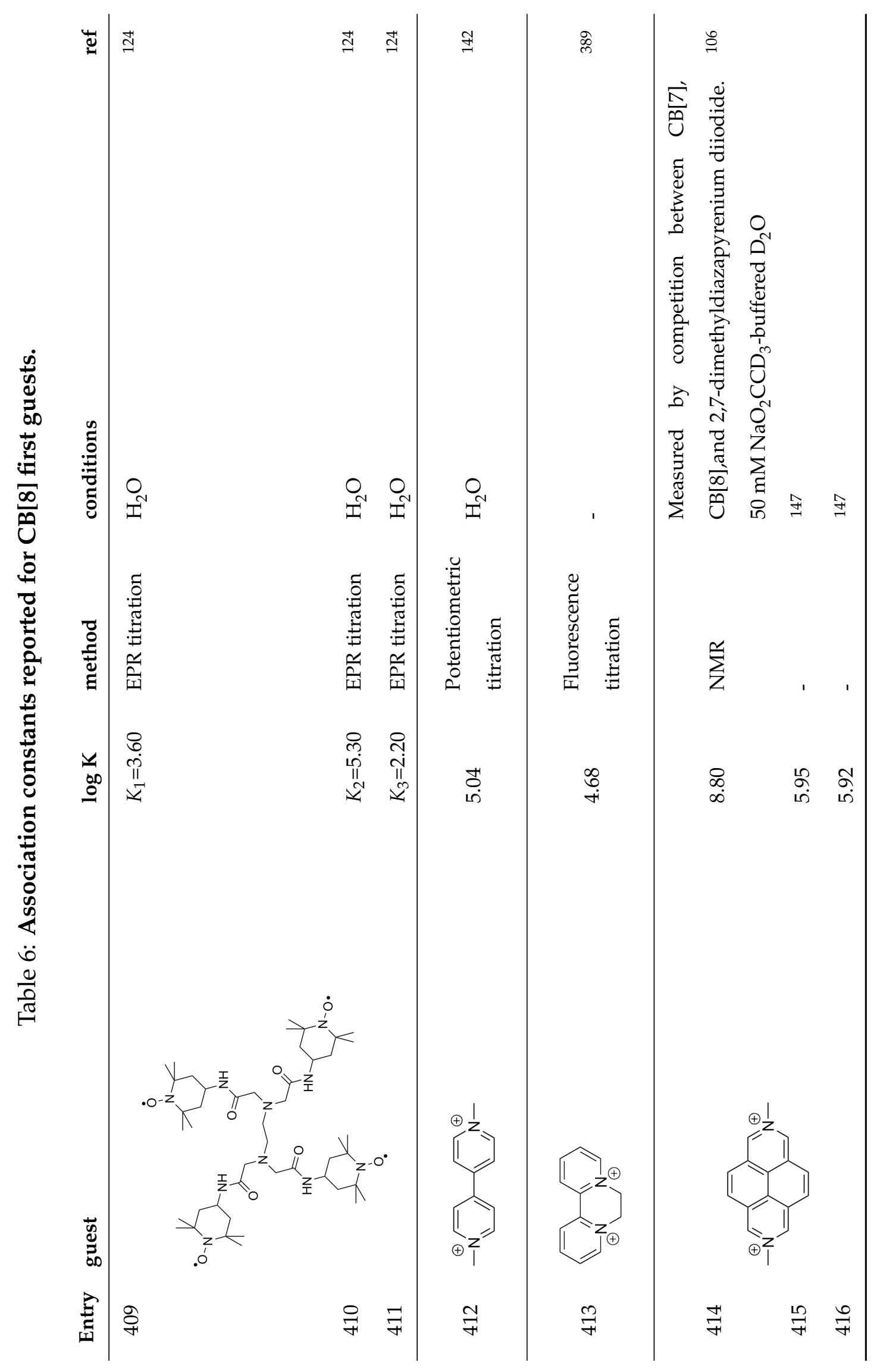




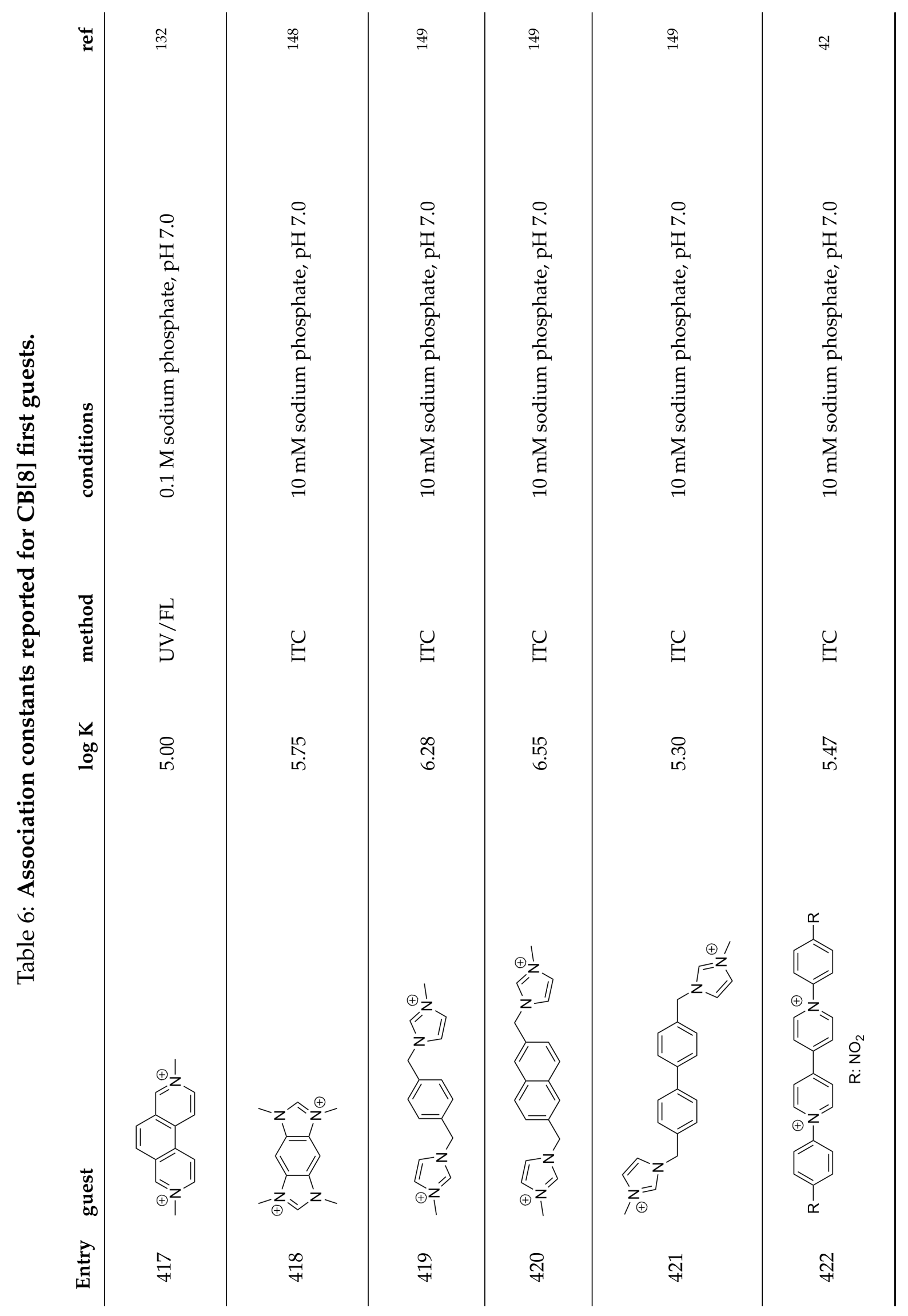



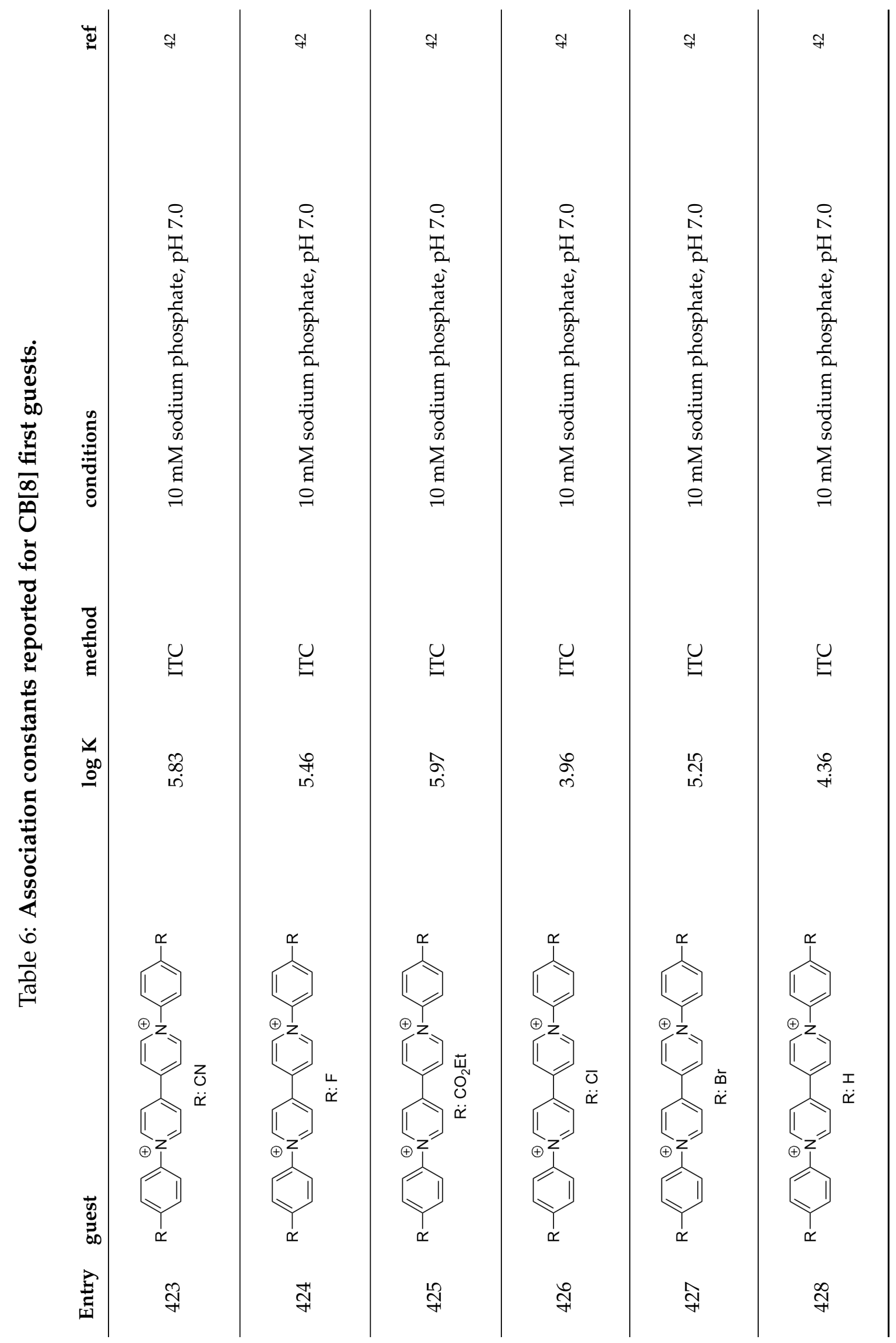

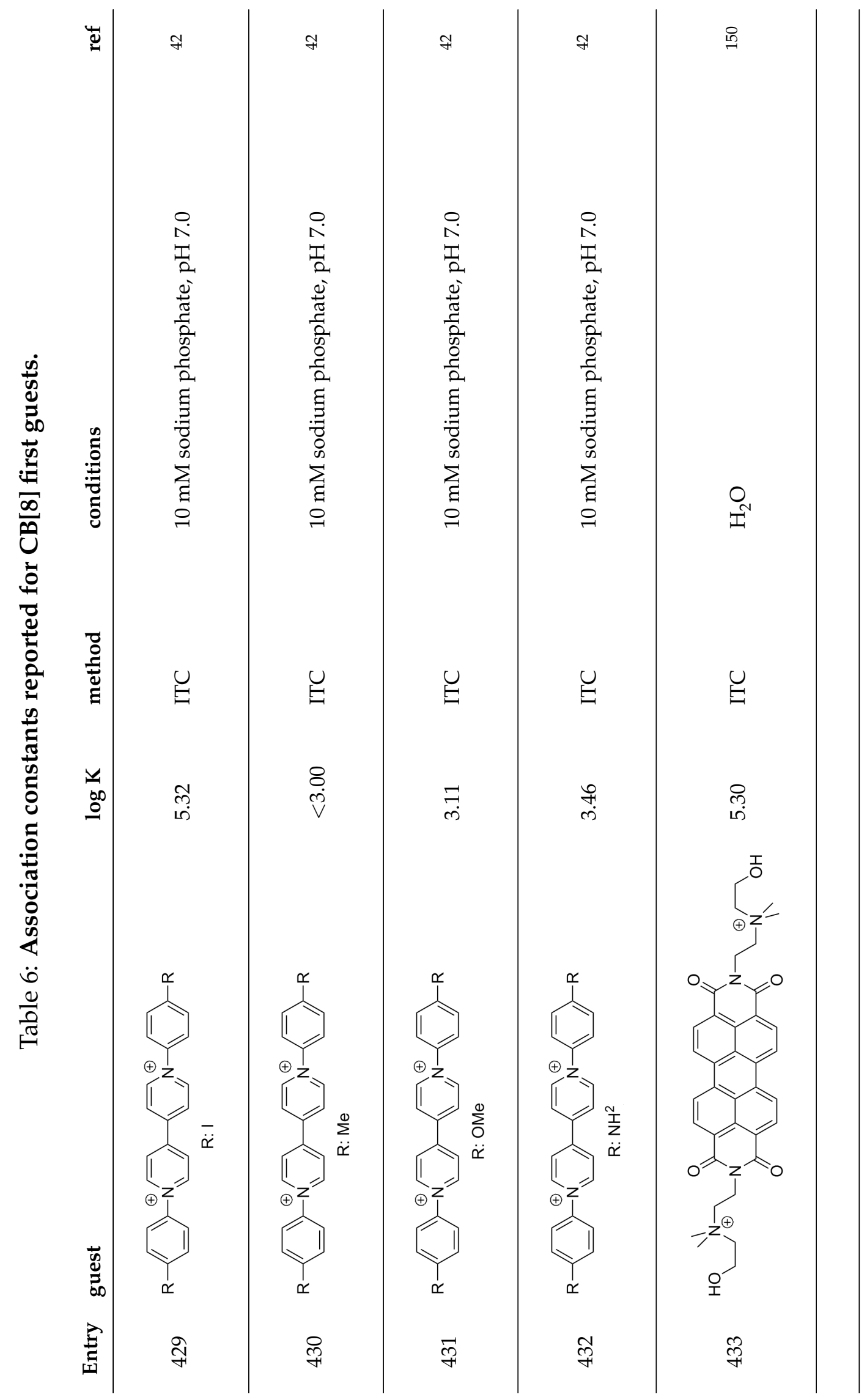


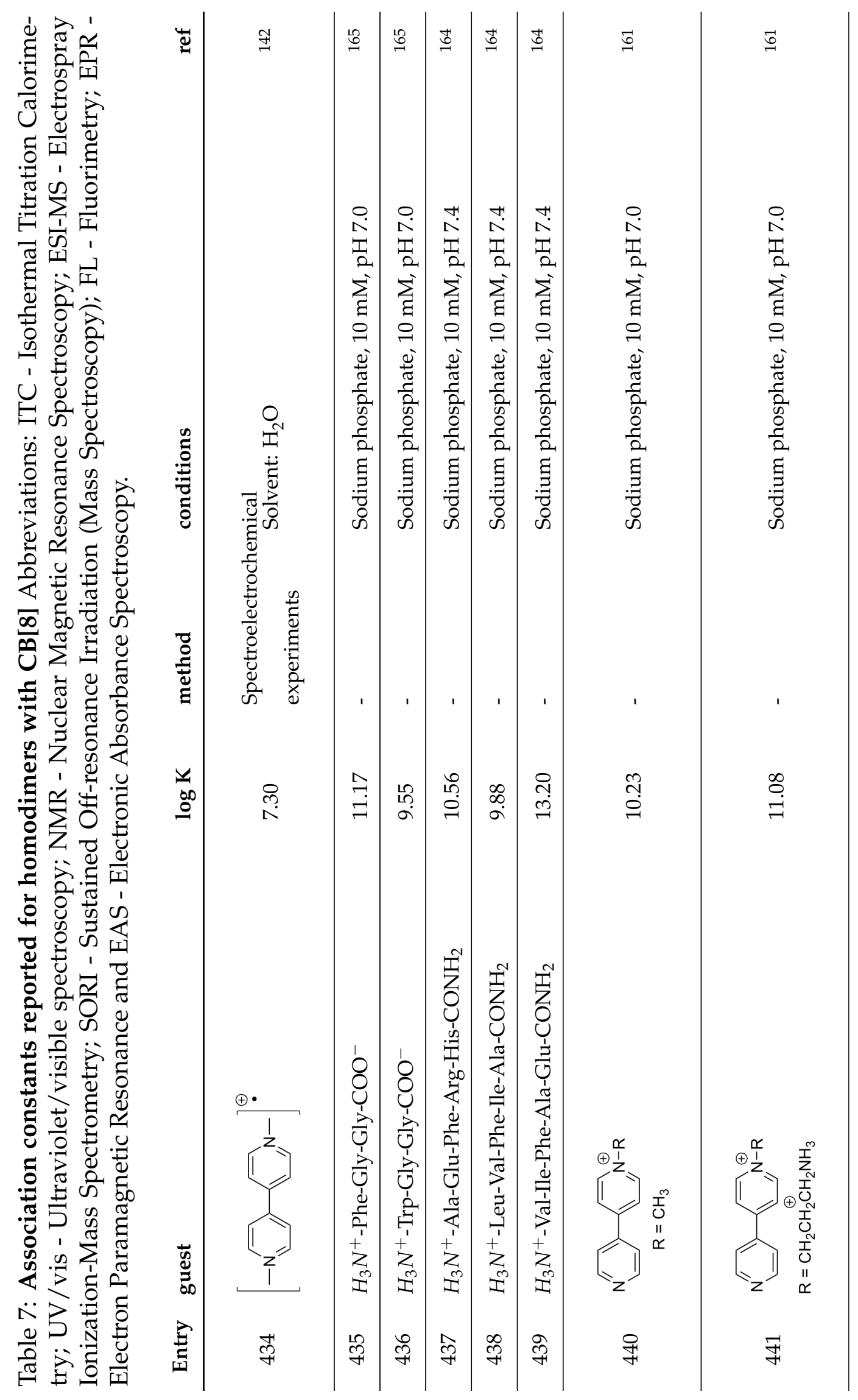




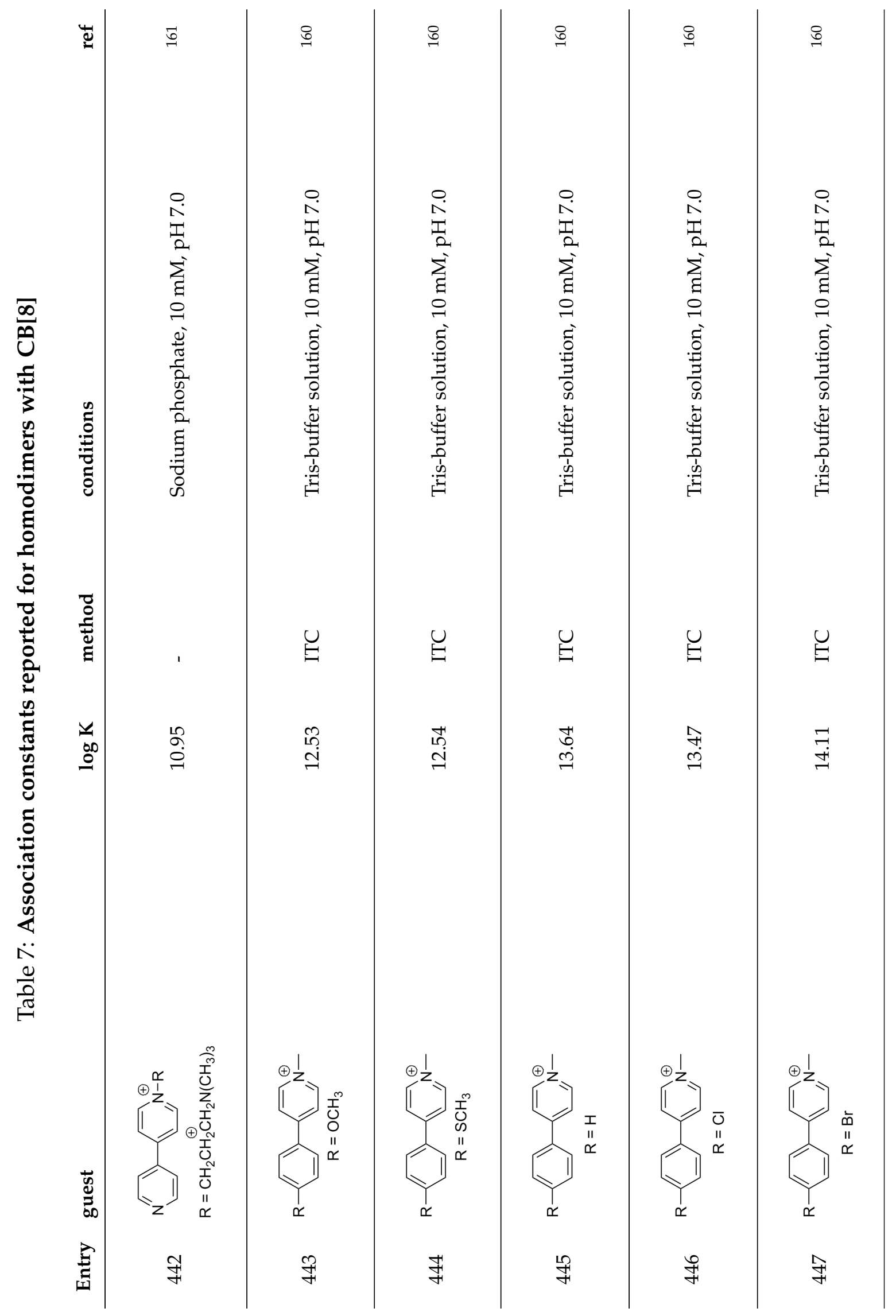




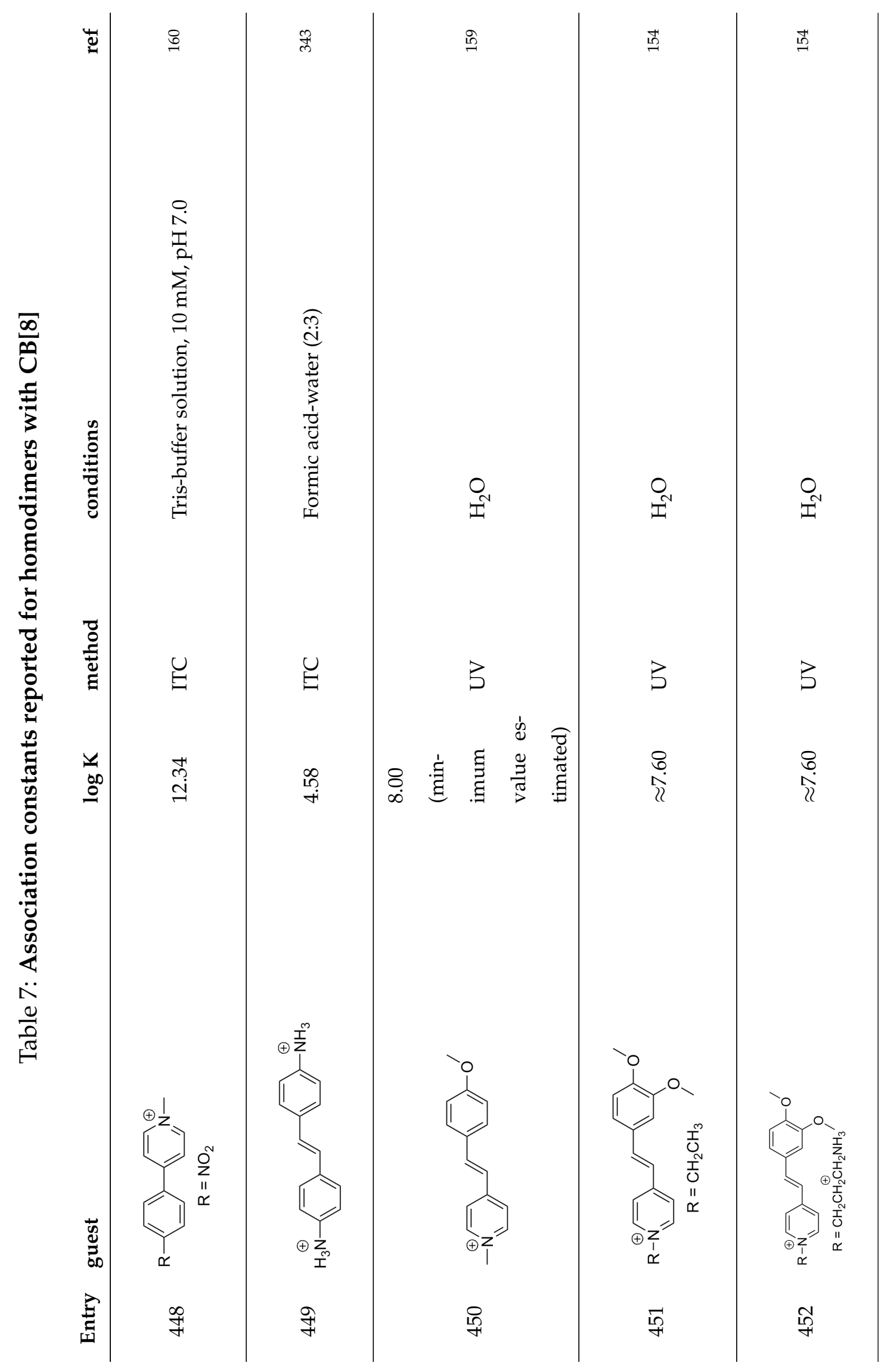




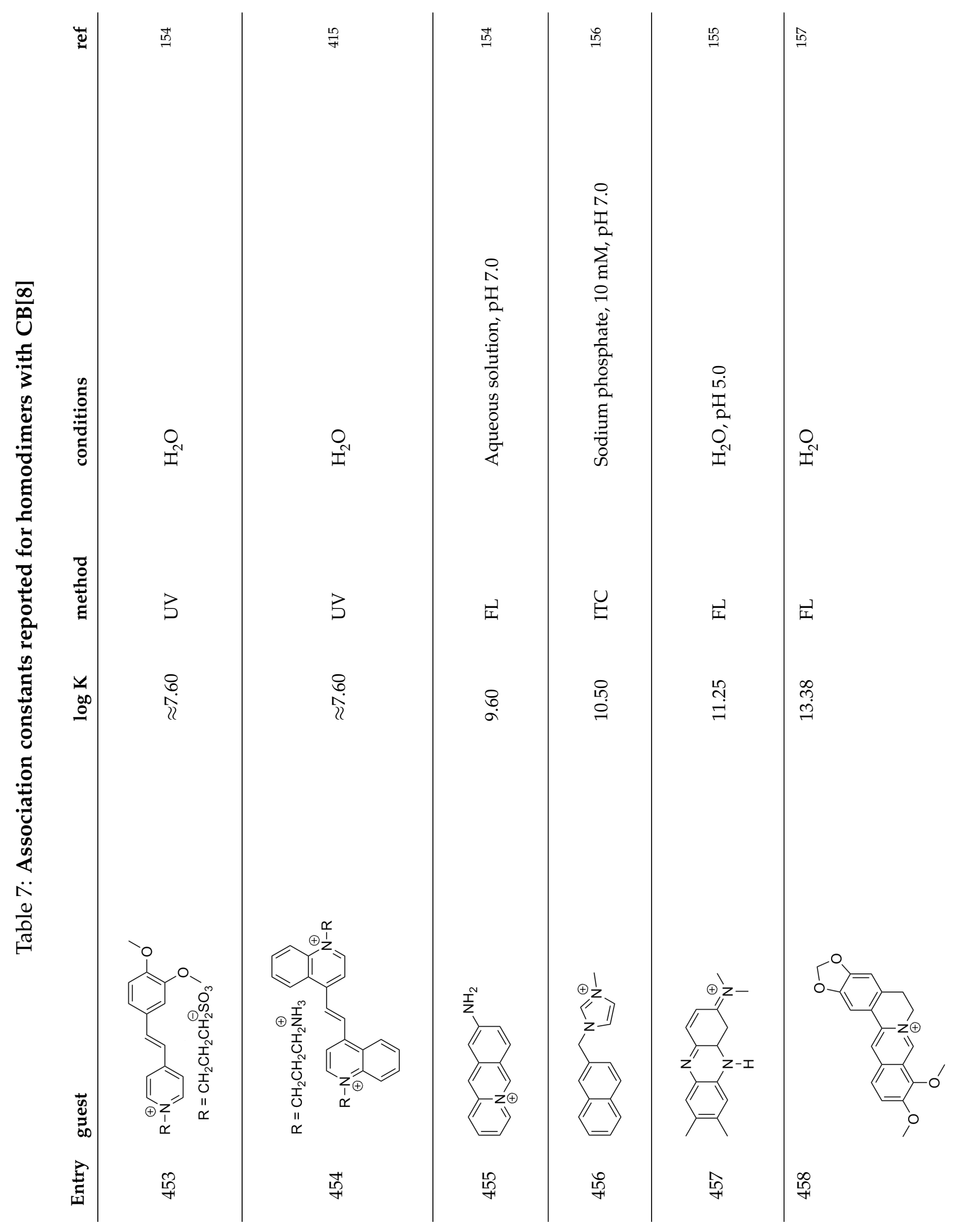




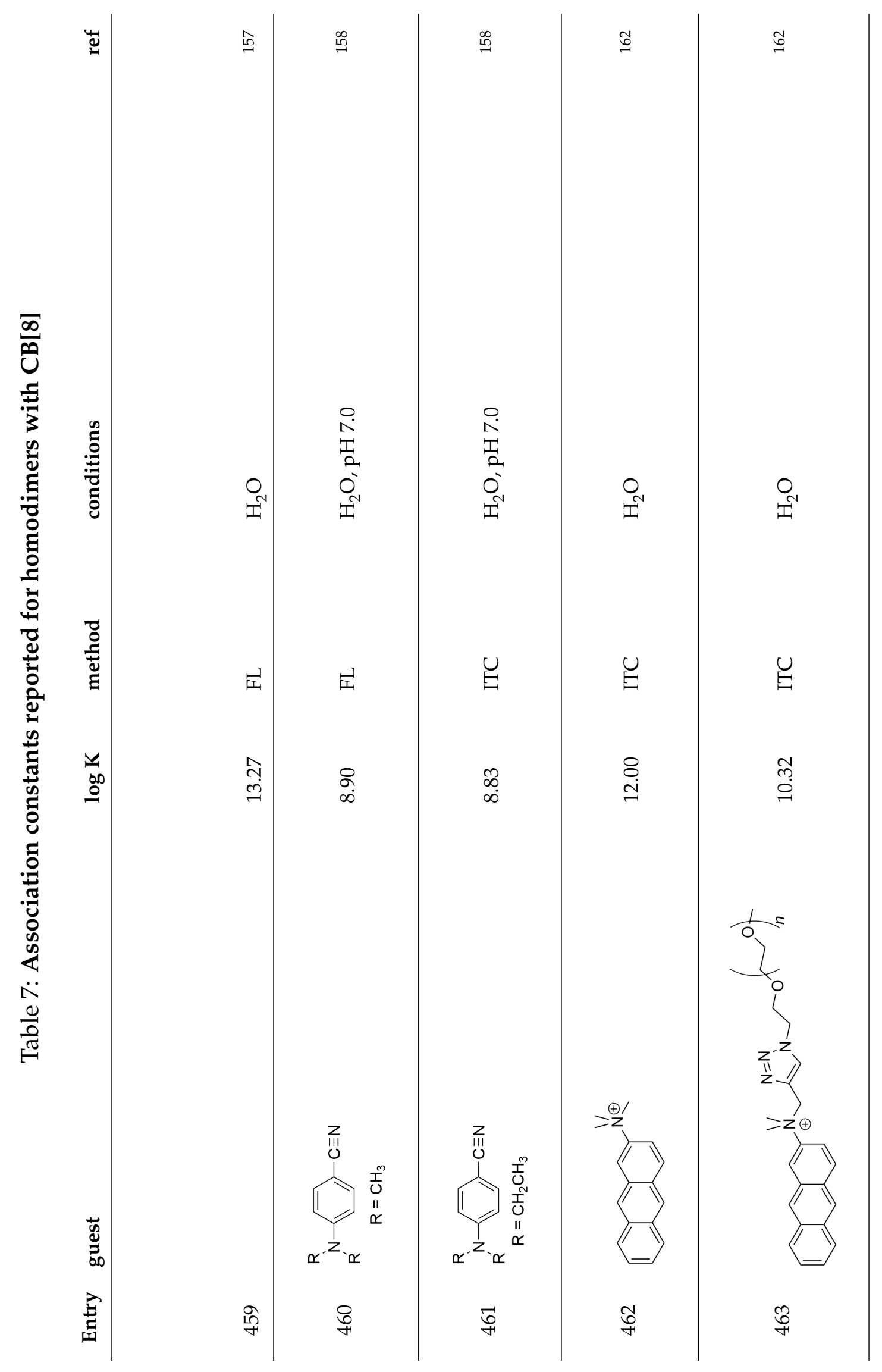




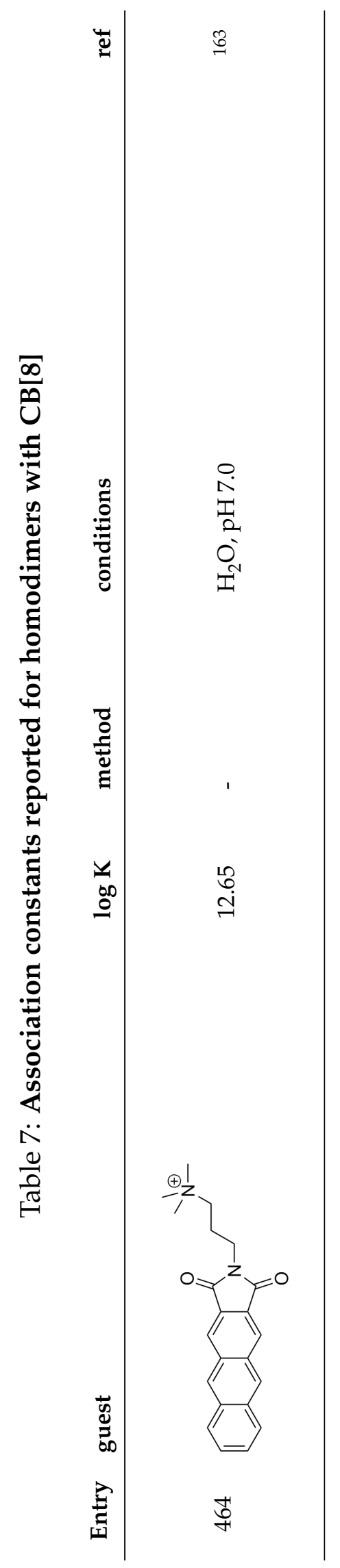




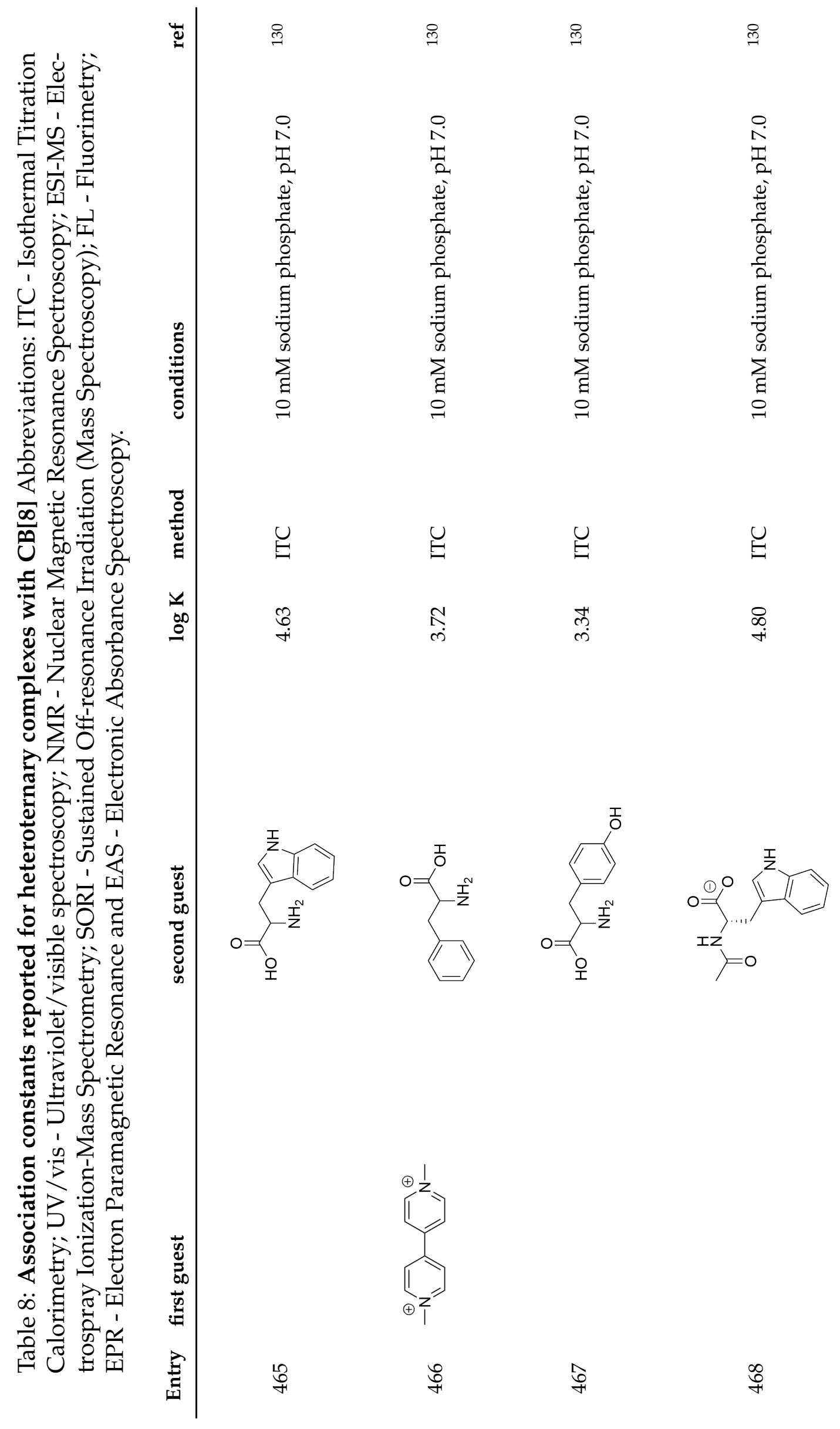




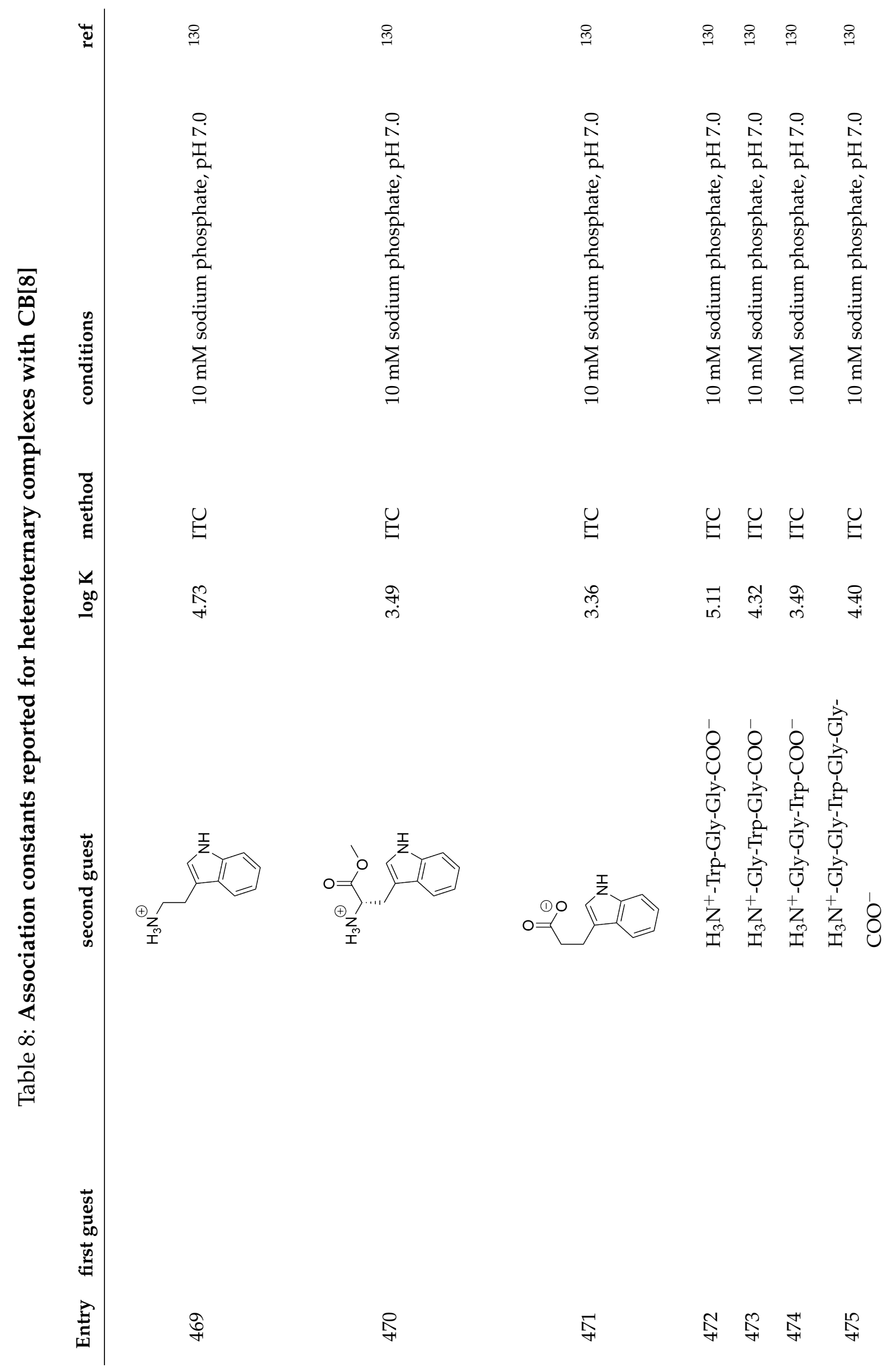




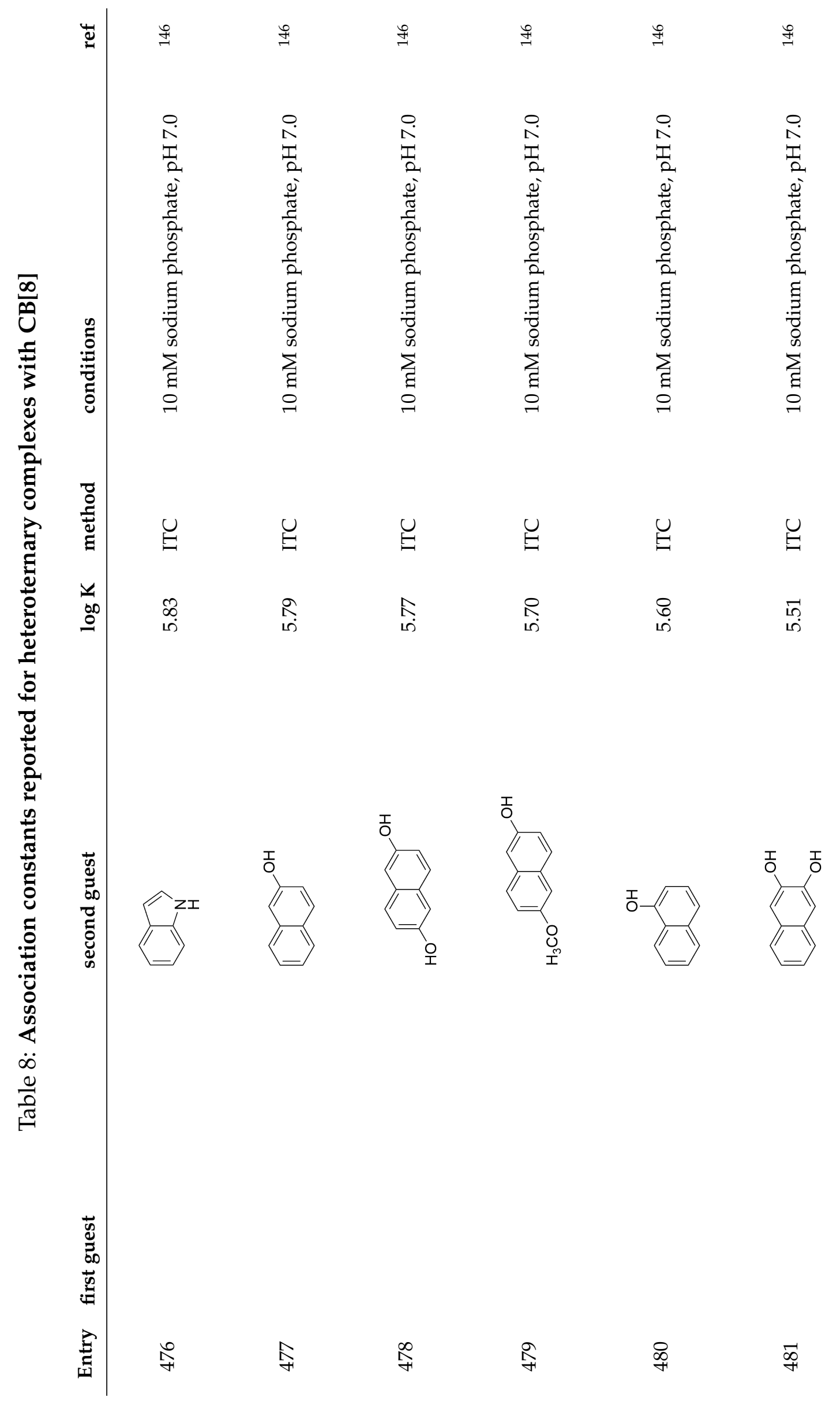




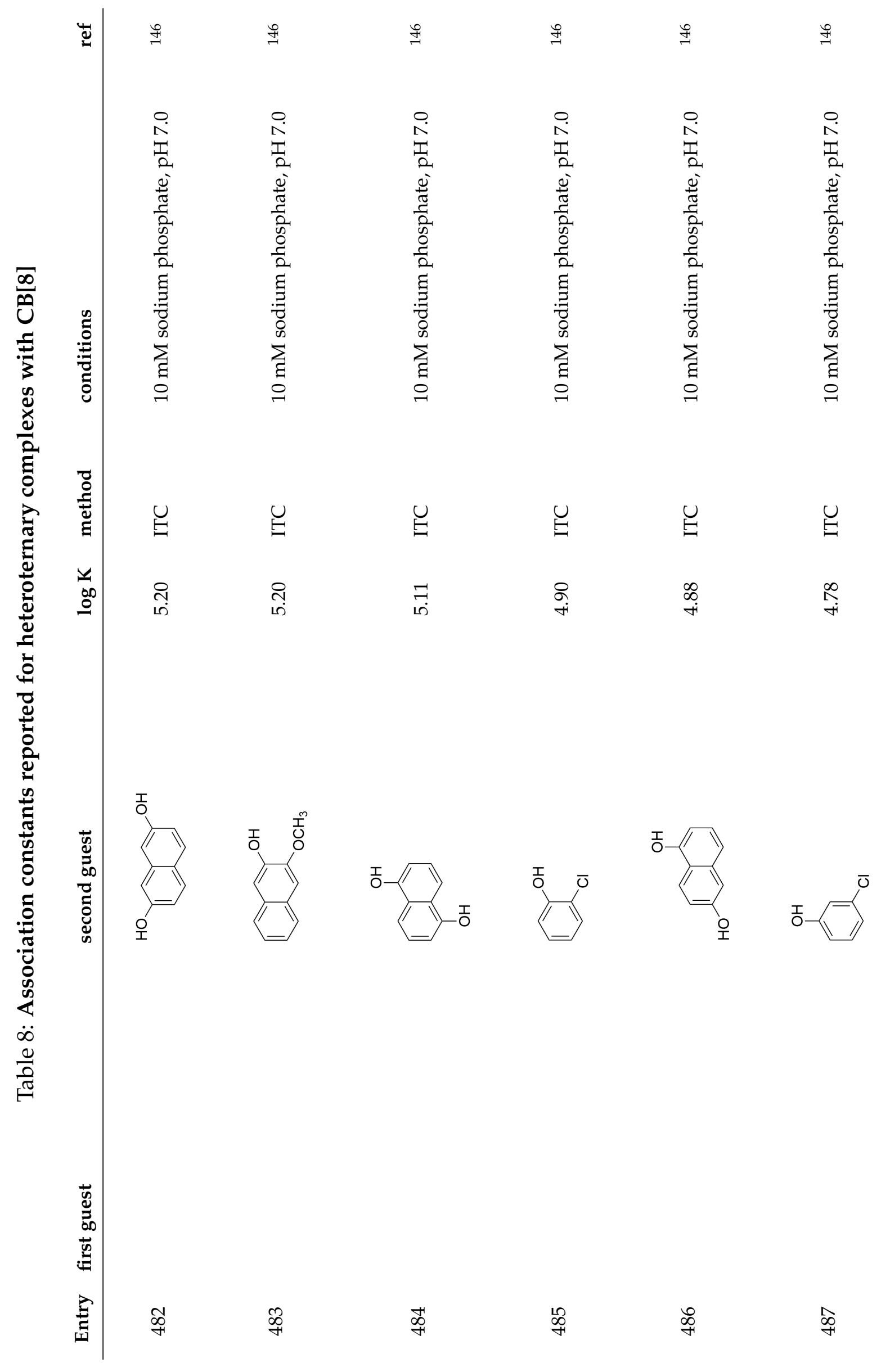




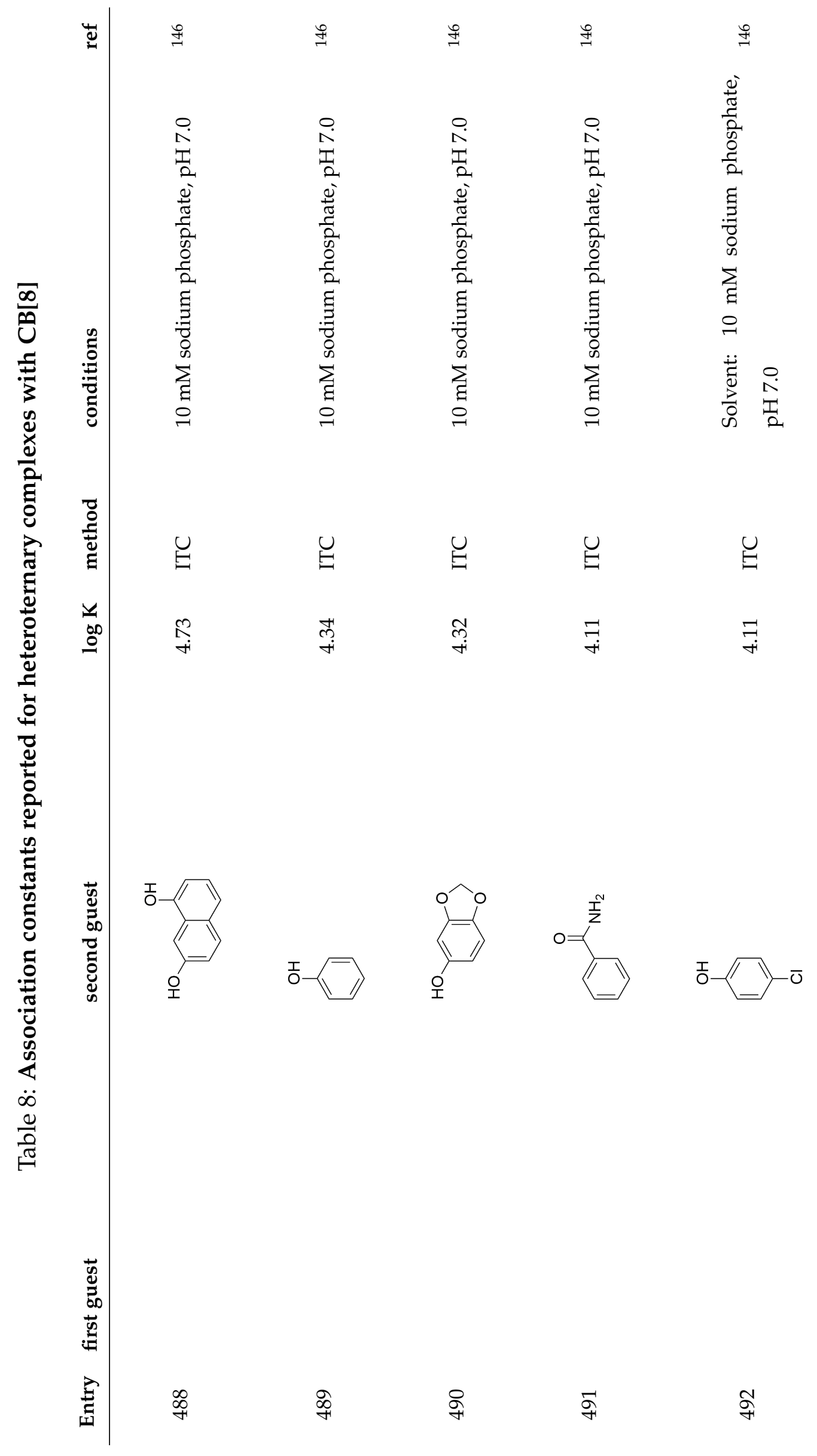




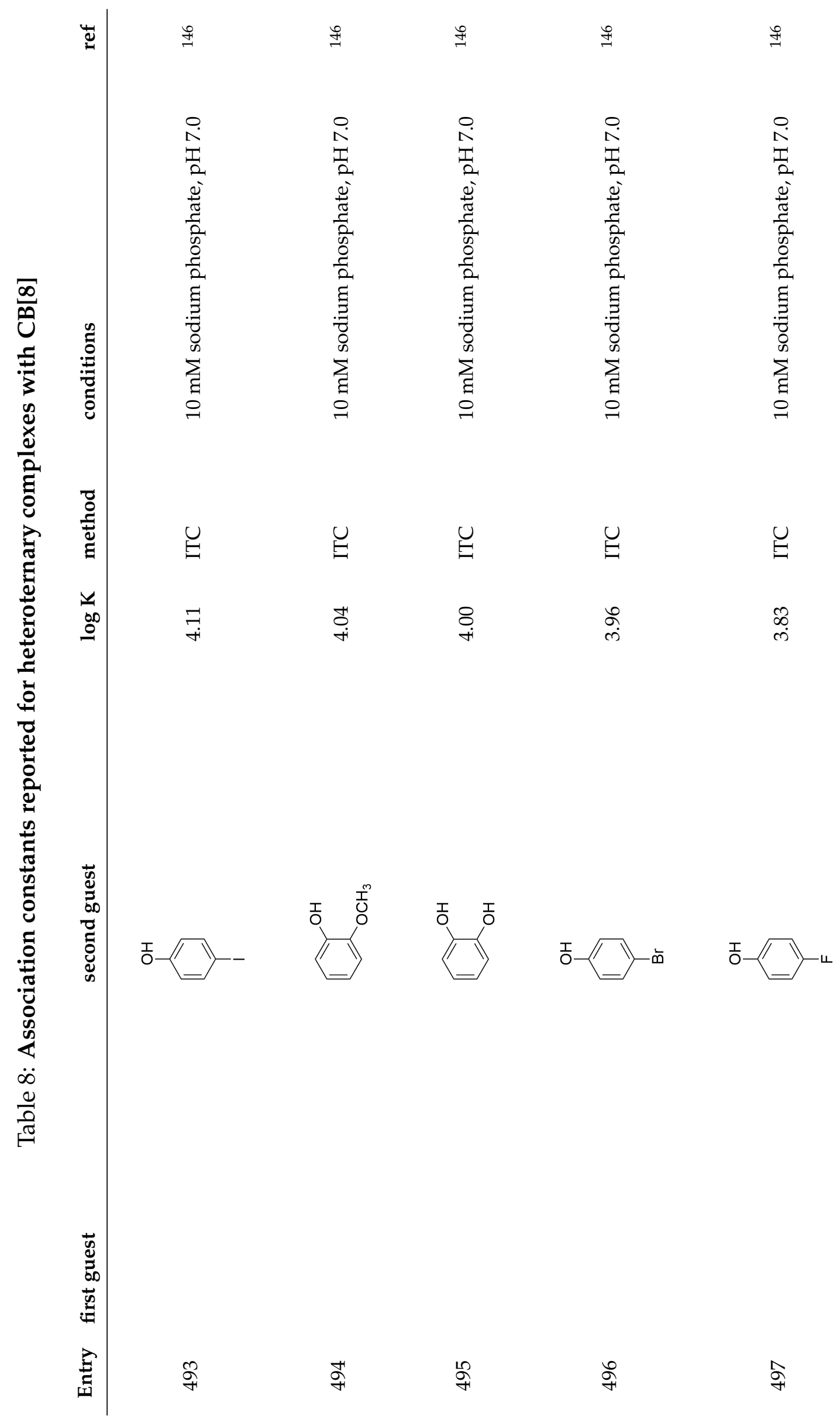




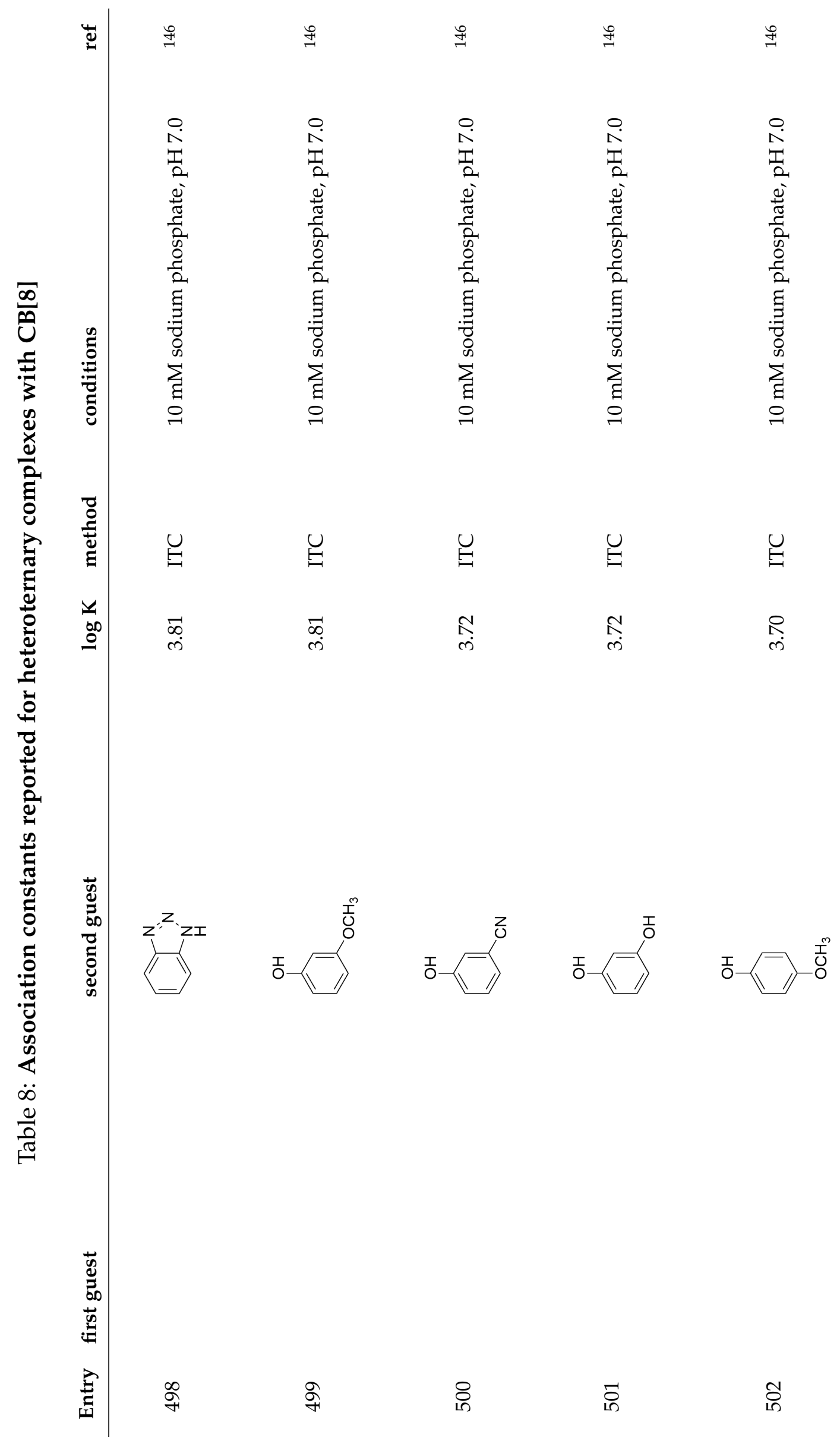




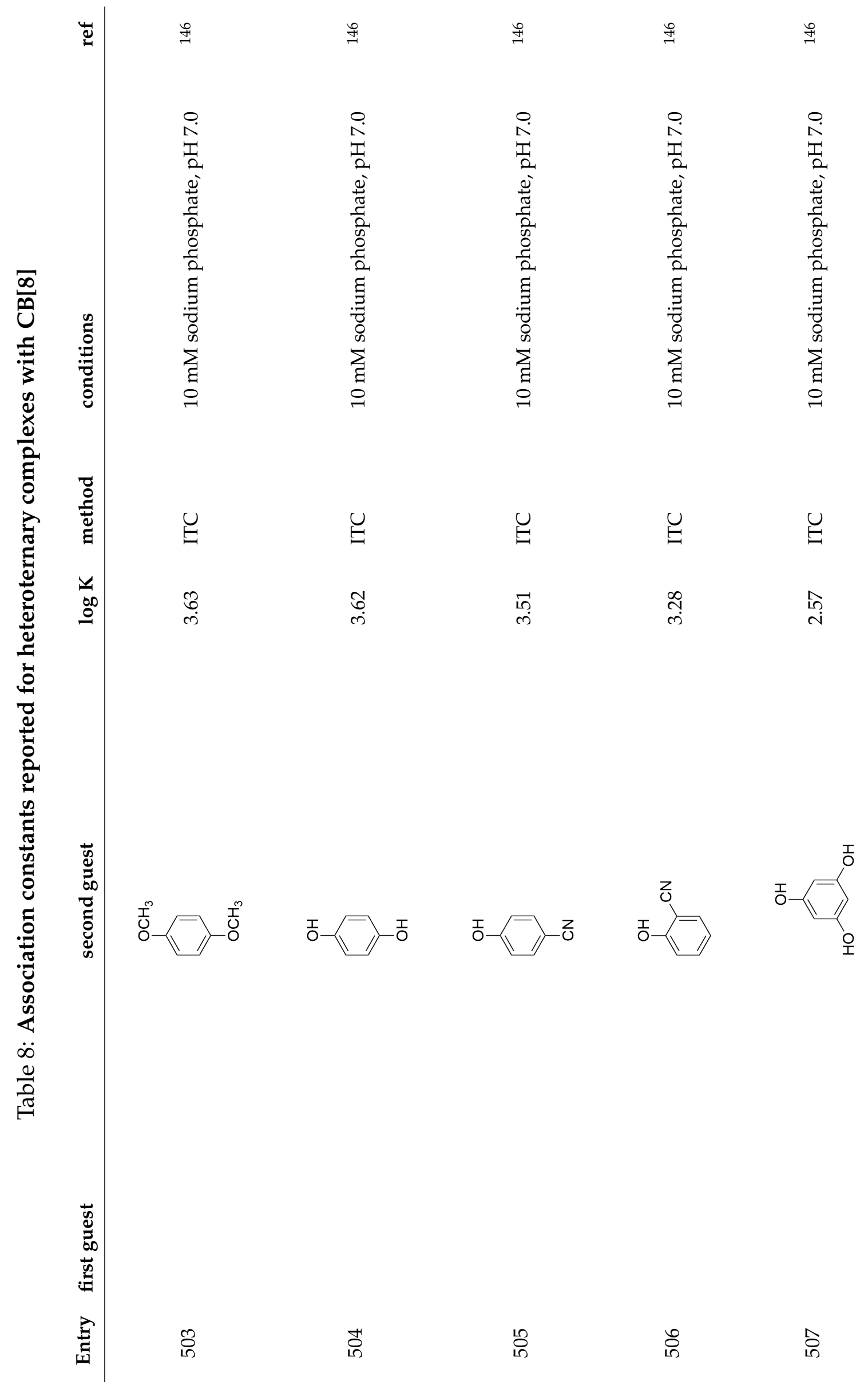




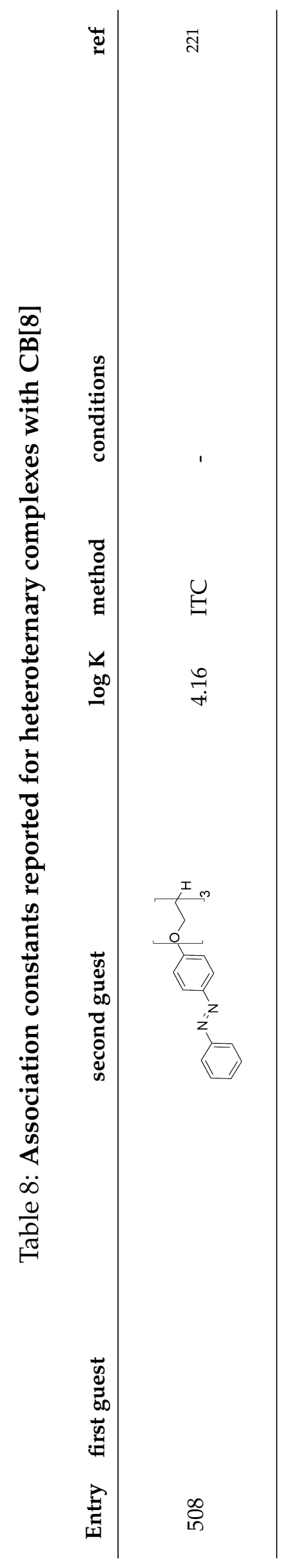




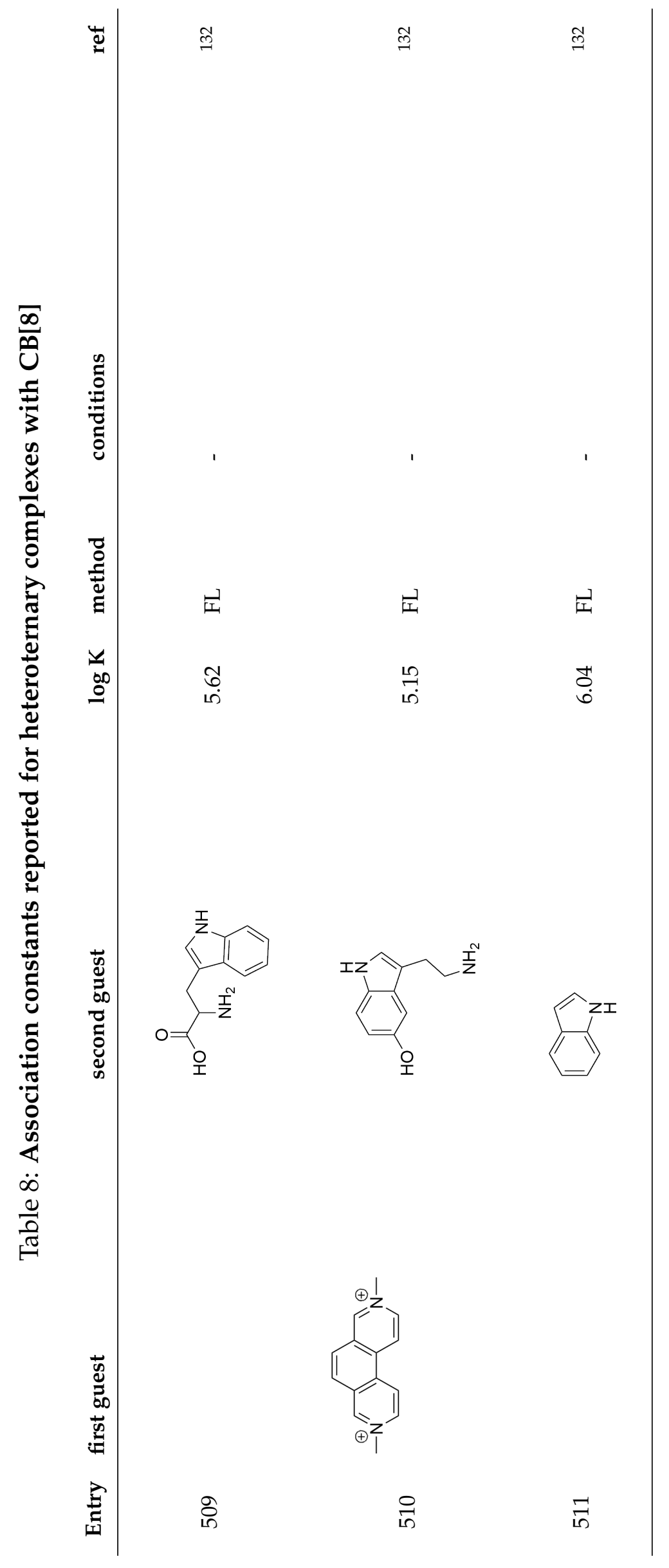




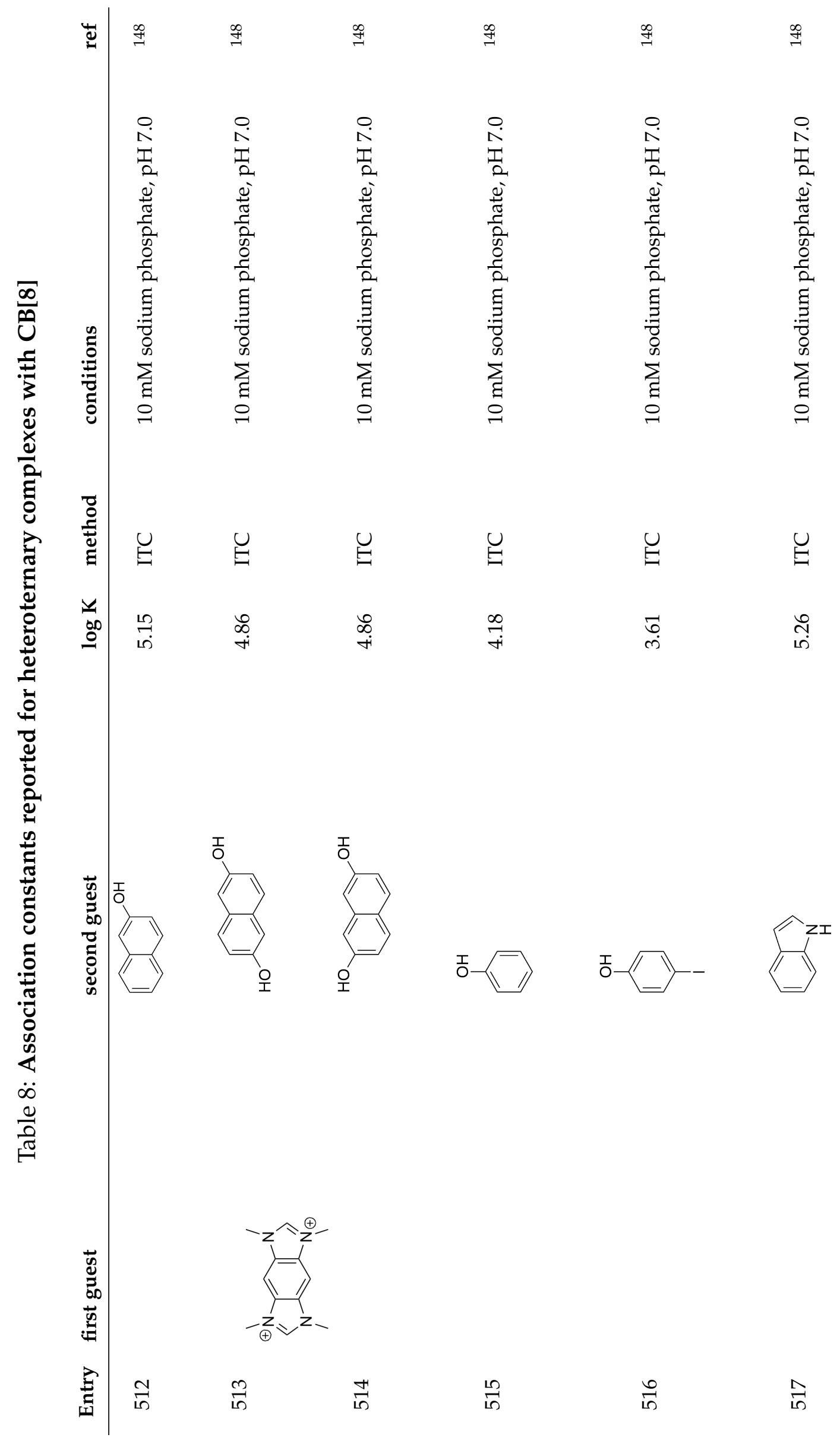




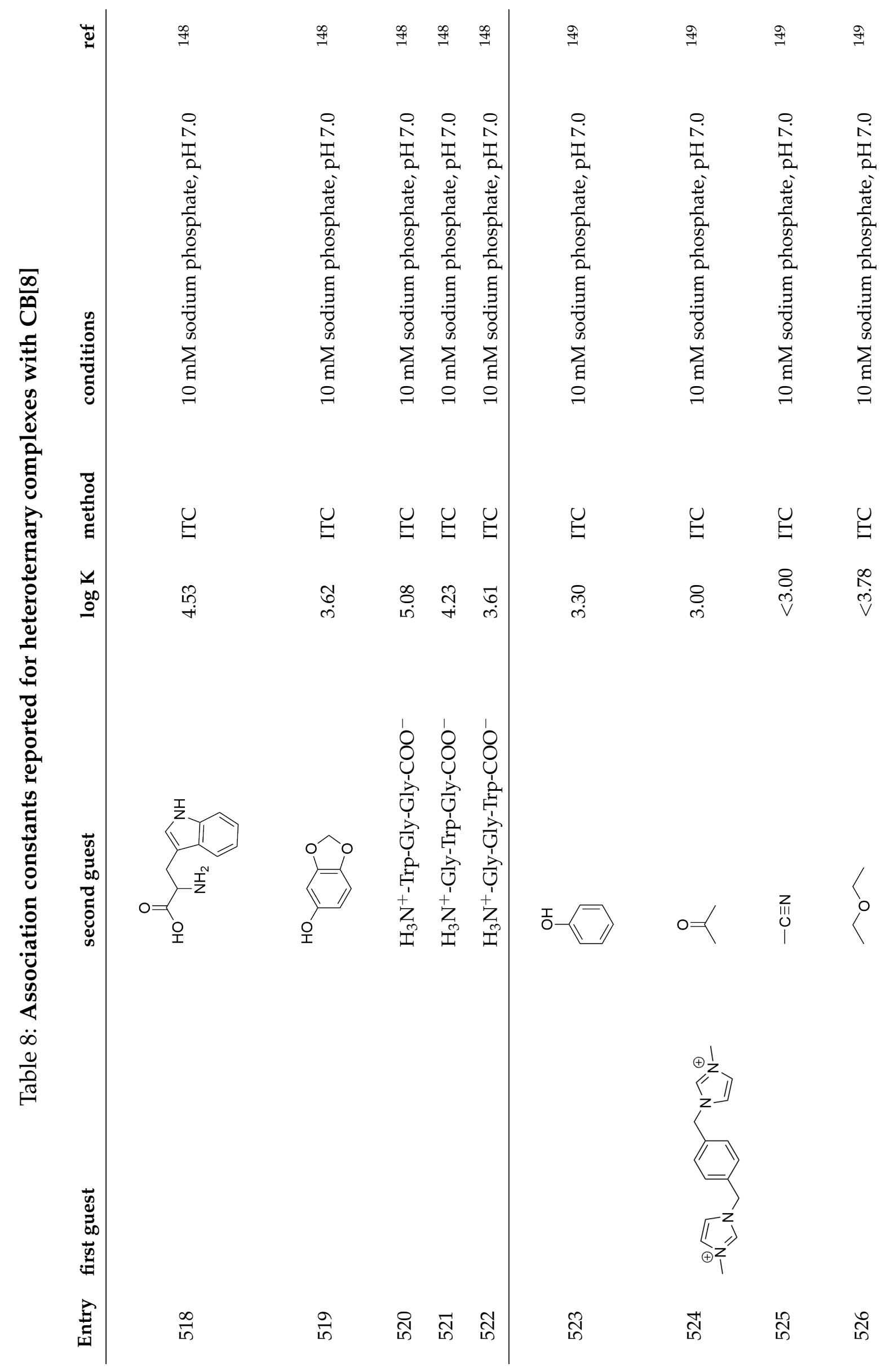




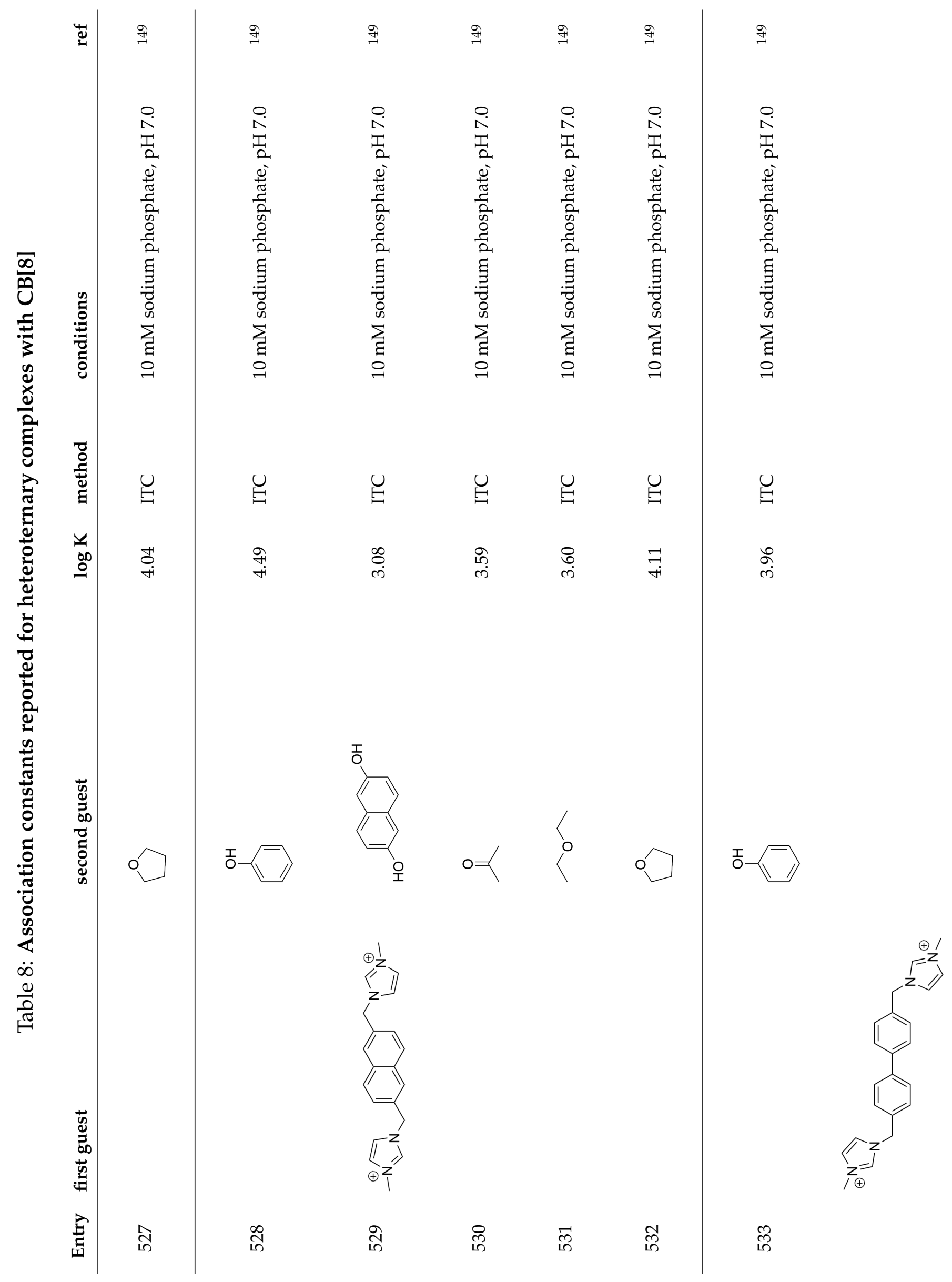




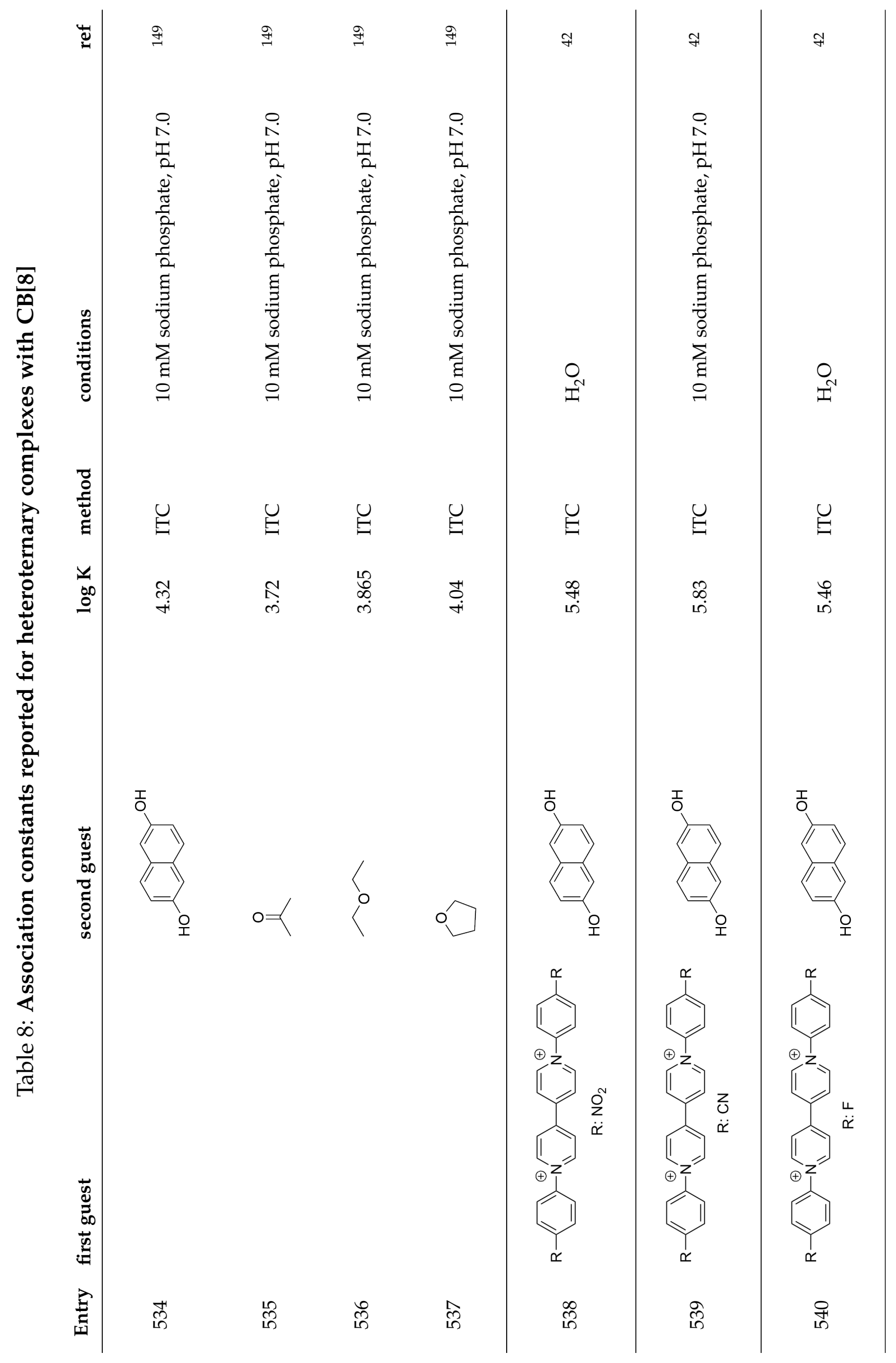




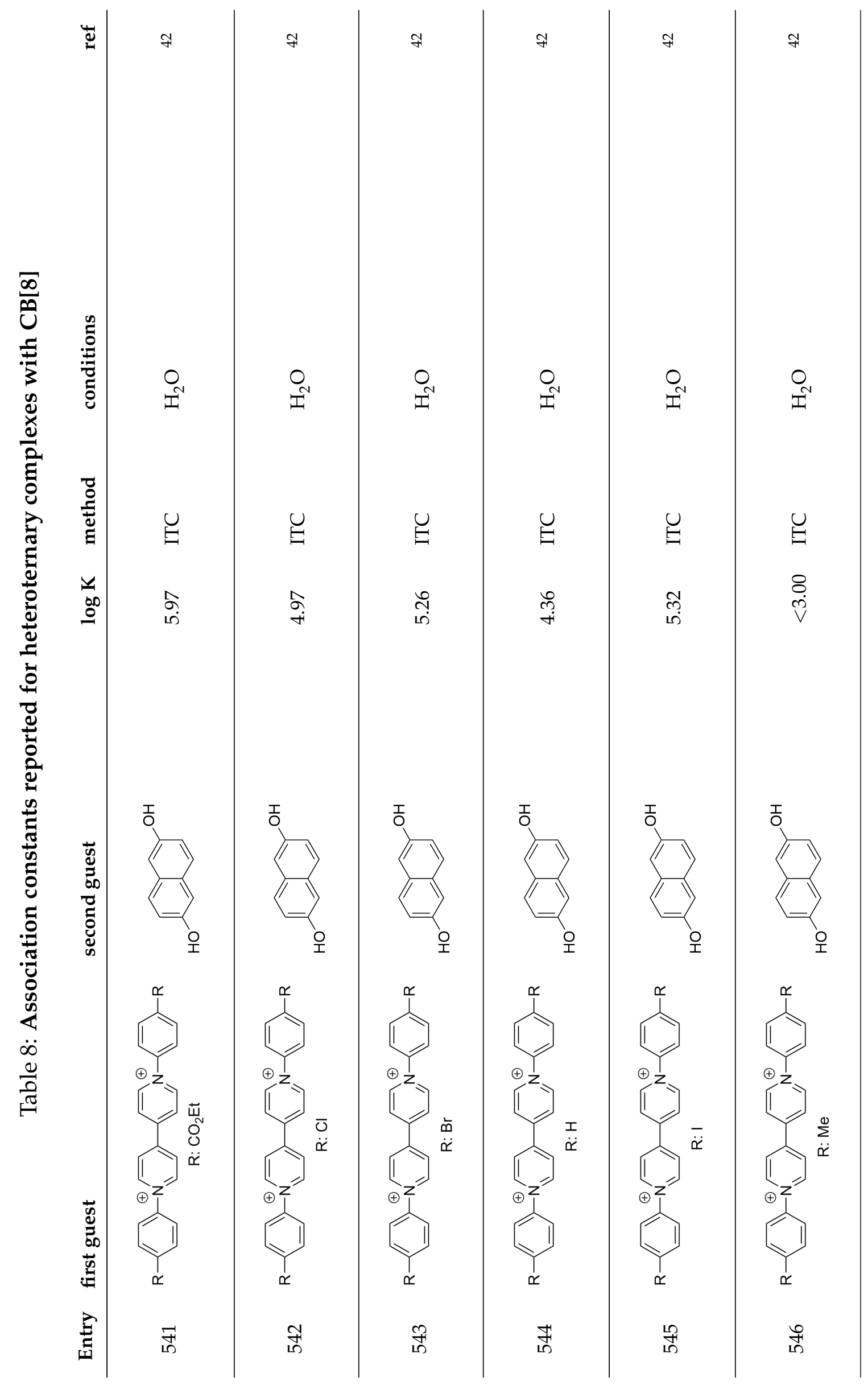




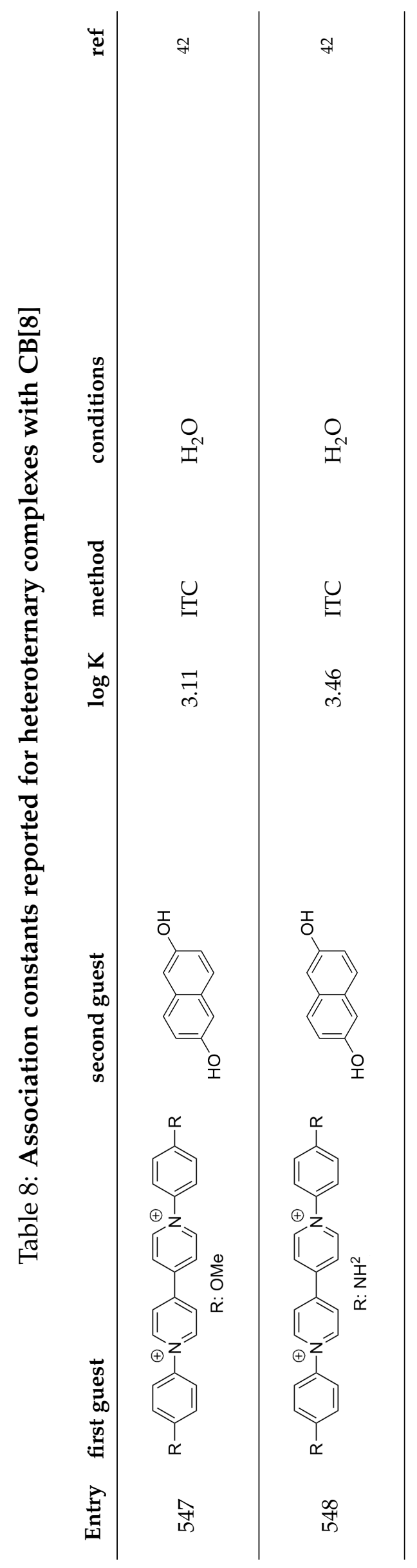




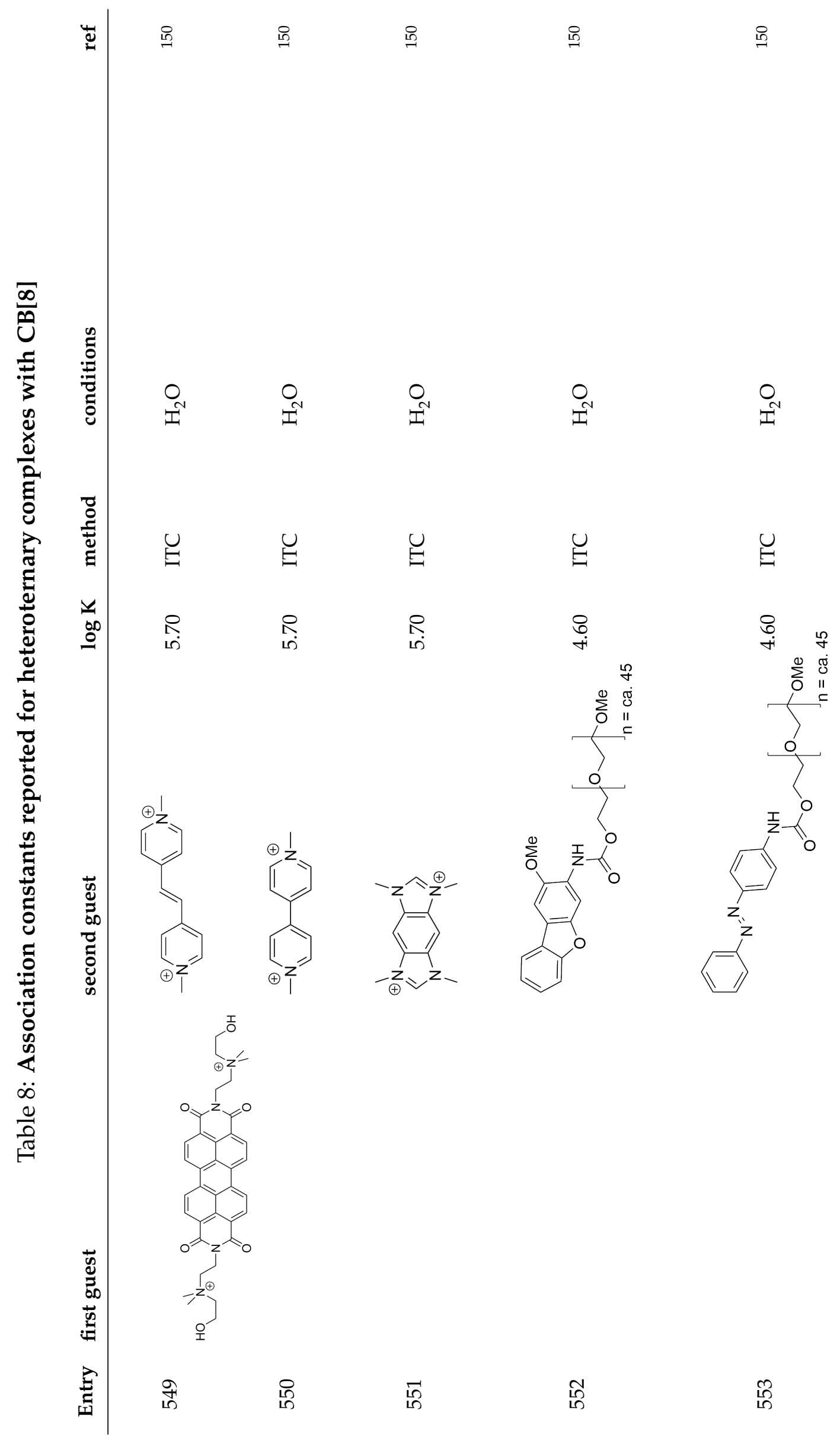




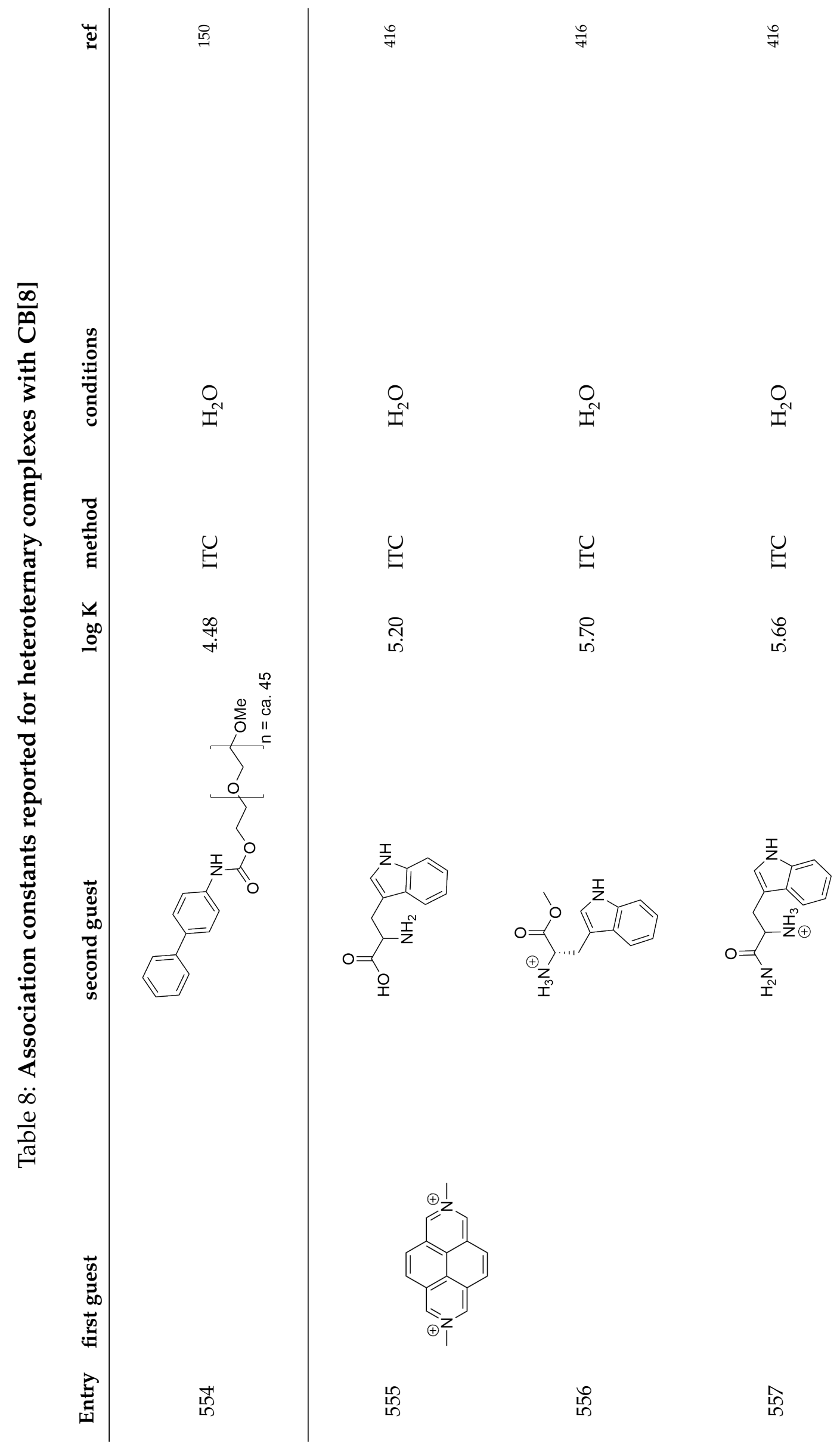




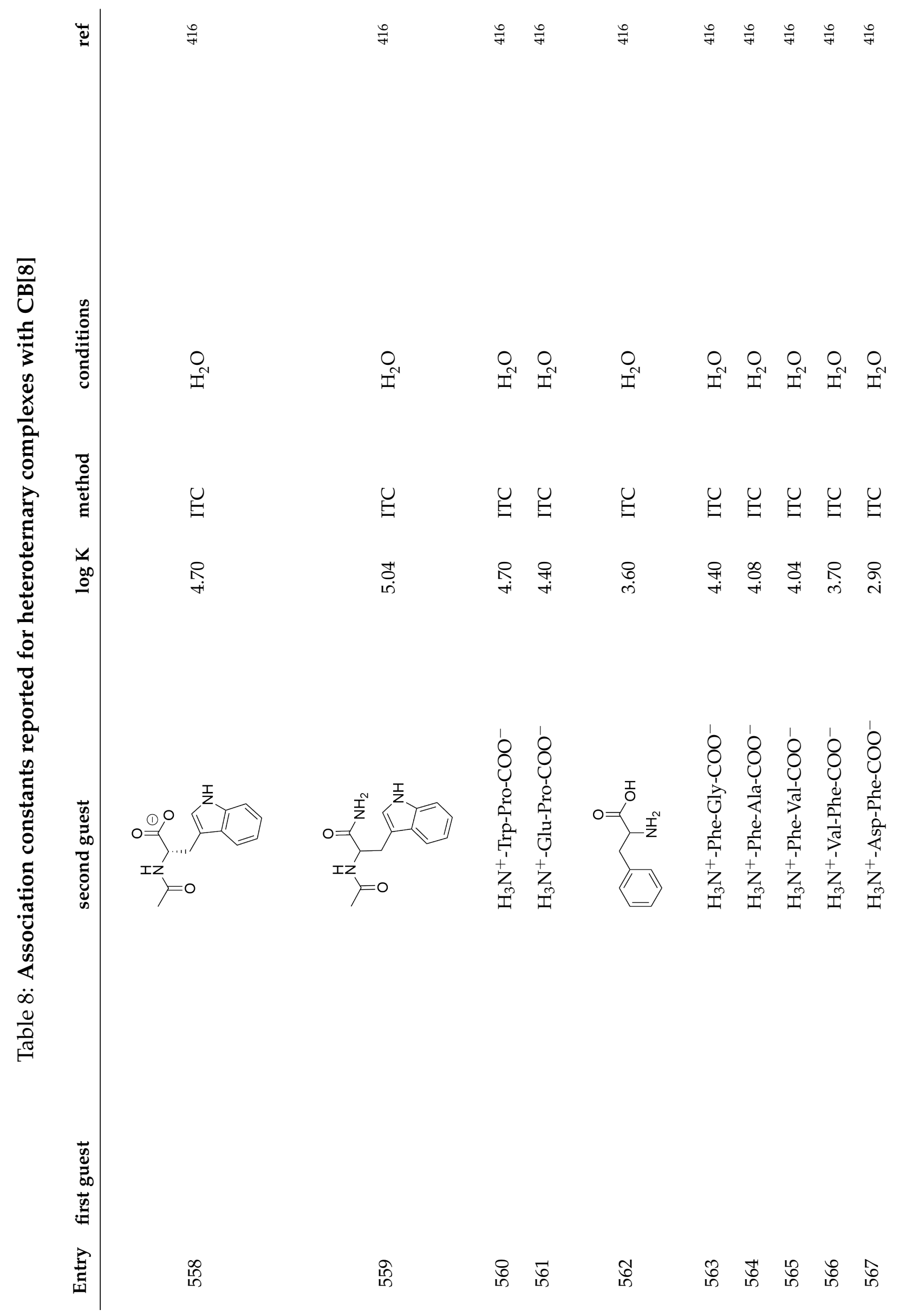




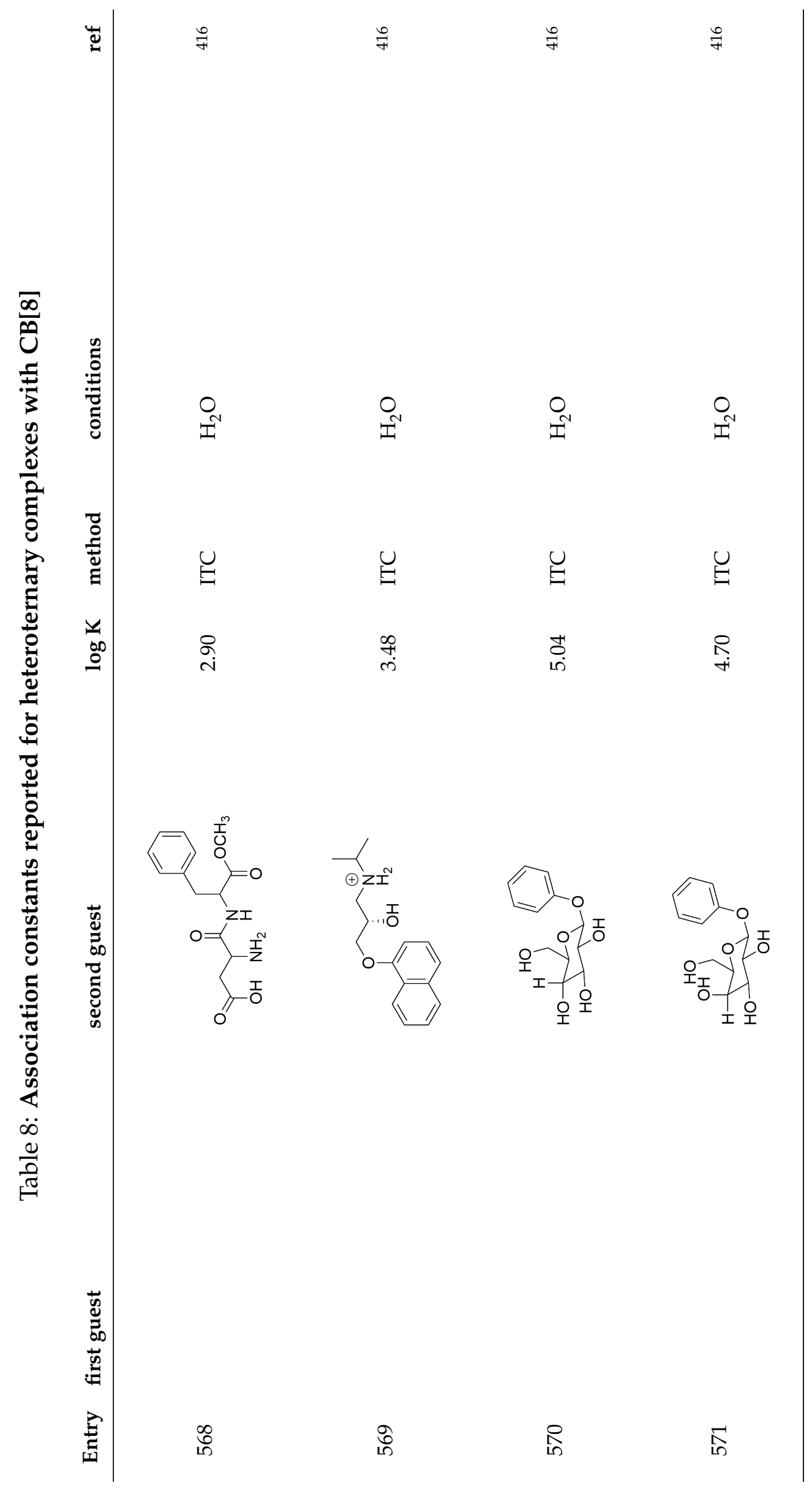




\section{Biographies}

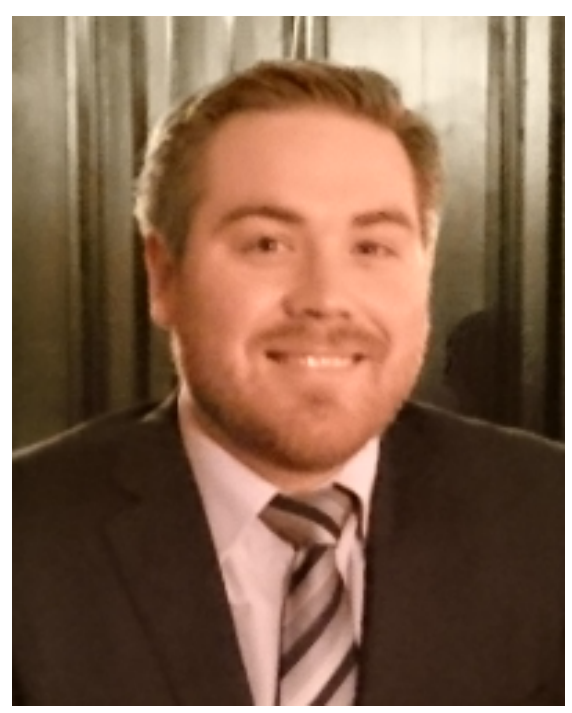

Steven Barrow received his BSc degree in Nanotechnology from the Royal Melbourne Institute of Technology (RMIT) in Australia, and completed his PhD under the supervision of Prof. Paul Mulvaney at the University of Melbourne in 2013. Steven's PhD research involved investigating the optical properties of self-assembled gold nanostructures using Dark-Field Microscopy. In 2014, Steven began a postdoctoral position with Prof. Oren Scherman in the Melville laboratory for polymer synthesis at the University of Cambridge, and was awarded a Marie Curie Fellowship in 2015. Steven's current research concerns utilising nanoparticle-cucurbit $[n]$ uril constructs for the dynamic assembly of nanoparticles, as well as monitoring chemical reactions and gas sensing. 


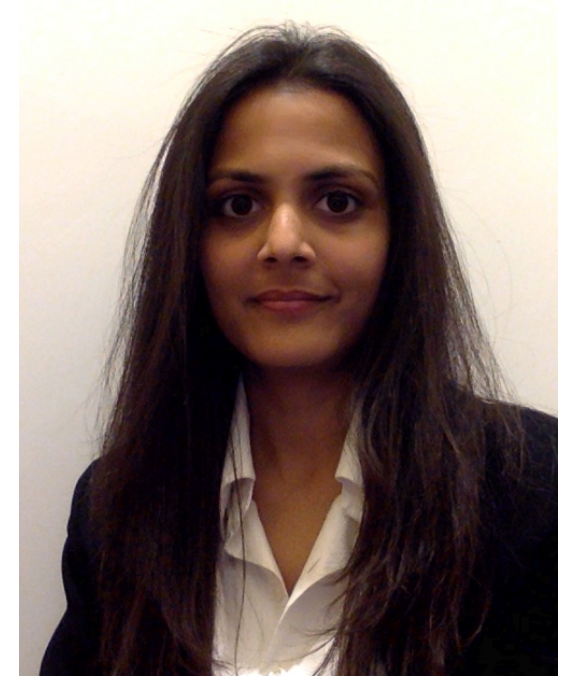

Setu Kasera received her BSc degree in Biological Chemistry from Aston University, UK. She then joined Prof. Oren A. Scherman's group at the University of Cambridge as an MPhil candidate and continued as a PhD student. Her research focuses on plasmonic constructs with cucurbit $[n]$ urils for sensing applications using surface-enhanced Raman spectroscopy.

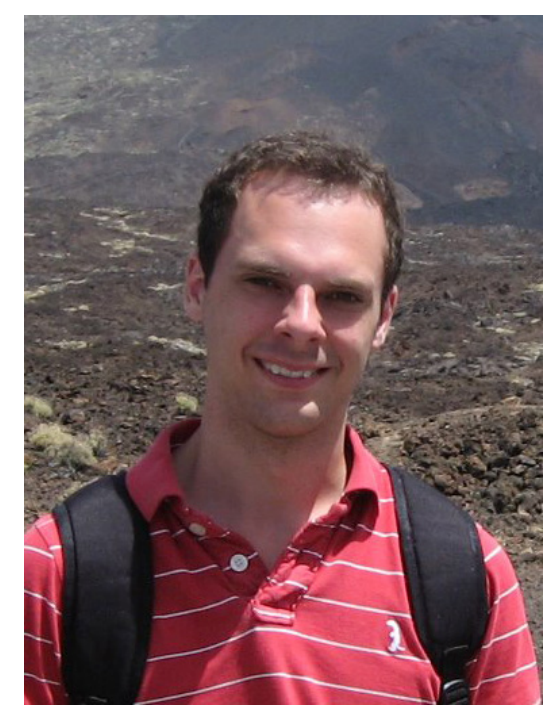

Matthew Rowland graduated from the University of Warwick UK in 2011 with an MChem degree in Chemistry with Industrial Training. He then joined the Cambridge University PhD Training Programme in Chemical Biology and Molecular Medicine and began research in the laboratory of Prof. Oren A. Scherman as a PhD student. Matthew's 
research focuses on the development of biocompatible hydrogels which self-assembly on account of utilisation of the host-guest chemistry of cucurbit[ $n]$ uril. His specific interests lie in healthcare and the delivery of therapeutic agents from soft materials.

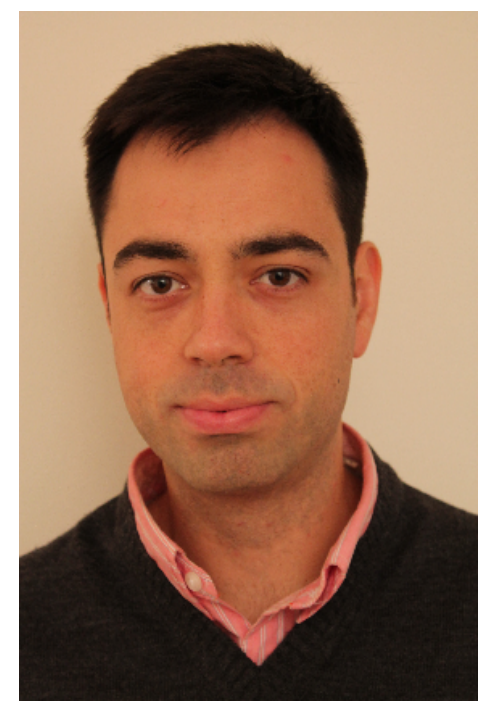

Jesús del Barrio studied chemistry at the University of Zaragoza, Spain, and received his doctoral degree in 2010 with a thesis on photoresponsive polymers for optical applications. He then moved to the United Kingdom to work on supramolecular materials at The University of Cambridge with Professor Oren Scherman. His postdoctoral studies, supported in part by a Marie Curie Intra-European Research Fellowship, were in the area of host-guest interactions and macromolecular self-assembly in aqueous media. In 2014, he took up a position as research scientist at the Schlumberger Gould Research Centre in Cambridge, UK. His work encompasses both basic and applied research in the soft matter area aimed at developing innovative polymer fluids, viscoelastic systems and responsive materials. 


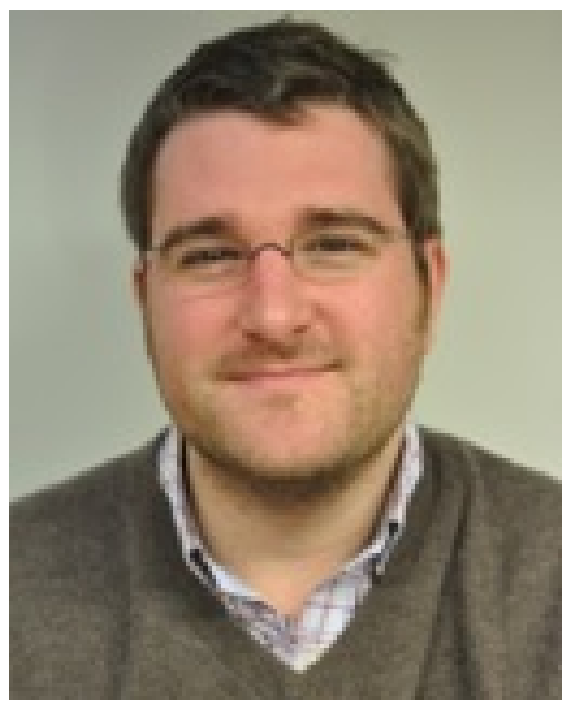

Oren Scherman graduated from Cornell University in Ithaca, New York, with a BA in Chemistry in 1999. He then moved to Pasadena, California, where he completed a PhD in 2004 in the area of olefin metathesis and controlled polymerisation, under the supervision of Professor Robert H. Grubbs at the California Institute of Technology (Caltech). After finishing his $\mathrm{PhD}$, Oren moved to the Netherlands to work on supramolecular polymers with Professors E.W. Meijer and Rint P. Sijbesma at the Eindhoven University of Technology. In 2006, he moved to the University of Cambridge to take up an academic appointment as a University Lecturer and Next Generation Fellow in the Melville Laboratory for Polymer Synthesis in the Department of Chemistry. In 2012, he was promoted to Reader in Supramolecular and Polymer Chemistry and in March 2013, he was appointed as the Director of the Melville Laboratory; Oren was promoted to Professor in 2015. During the 2013-2014 academic year, Oren was on sabbatical at Tsinghua University as the Xuetang Visiting Professor in Chemistry. His research group is interested in dynamic supramolecular self-assembly at interfaces. Oren's current research projects include the application of macrocyclic host-guest chemistry using cucurbit $[n]$ urils in the development of novel supramolecular hydrogels and microcapsules, drug-delivery systems based on dynamic hydrogels, the conservation and restoration of important historical artefacts through the exploitation of supramolecular polymer chemistry and sensing and catalysis using self- 
assembled nanophotonic systems.

\section{Acknowledgement}

The authors thank all members of the Scherman group for help compiling the tables and proof-reading the review. S.J.B thanks the European Commission for a Marie Curie Fellowship (NANOSPHERE, 658360); S.K. thanks The Biochemical Society for the Krebs Memorial Scholarship and the Cambridge Commonwealth Trust for financial support. M.J.R thanks the University of Cambridge PhD Training Programme in Chemical Biology and Molecular Medicine; J.d.B. is grateful for a Marie Curie Intra-European Fellowship (Project 273807); we also acknowledge support from an ERC Starting Investigator Grant (ASPiRe, 240629) and a Next Generation Fellowship provided by the Walters-Kundert Foundation.

\section{References}

(1) Meyer, E. Uber die Condensation des Harnstoffs mit Glyoxal und des Glykolurils mit Formaldehyd. Heidelberg. Ph.D. thesis, Heidelberg University, Heidelberg, 1904.

(2) Behrend, R.; Meyer, E.; Rusche, F. I. Ueber Condensationsproducte aus Glycoluril und Formaldehyd. Ph.D. thesis, Heidelberg University, 1905.

(3) Freeman, W. A.; Mock, W. L.; Shih, N. Y. Cucurbituril. J. Am. Chem. Soc. 1981, 103, 7367-7368.

(4) Kim, J.; Jung, I.-S.; Kim, S.-Y.; Lee, E.; Kang, J.-K.; Sakamoto, S.; Yamaguchi, K.; Kim, K. New Cucurbituril Homologues: Syntheses, Isolation, Characterization, and X-ray Crystal Structures of Cucurbit[n]uril ( $\mathrm{n}=5,7$, and 8). J. Am. Chem. Soc. 2000, $122,540-541$. 
(5) Day, A.; Arnold, A. P.; Blanch, R. J.; Snushall, B. Controlling Factors in the Synthesis of Cucurbituril and Its Homologues. J. Org. Chem. 2001, 66, 8094-8100.

(6) Day, A. I.; Blanch, R. J.; Arnold, A. P.; Lorenzo, S.; Lewis, G. R.; Dance, I. A Cucurbituril-Based Gyroscane: A New Supramolecular Form. Angew. Chem. Int. Ed. 2002, 41, 275-277.

(7) Jon, S. Y.; Selvapalam, N.; Oh, D. H.; Kang, J.-K.; Kim, S.-Y.; Jeon, Y. J.; Lee, J. W.; Kim, K. Facile Synthesis of Cucurbit[n]uril Derivatives via Direct Functionalization: Expanding Utilization of Cucurbit[n]uril. J. Am. Chem. Soc. 2003, 125, 10186-10187.

(8) Zhao, N.; Lloyd, G. O.; Scherman, O. A. Monofunctionalised Cucurbit[6]uril Synthesis using Imidazolium Host-guest Complexation. Chem. Commun. 2012, 48, 30703072.

(9) Cao, L.; Isaacs, L. Daisy Chain Assembly Formed from a Cucurbit[6]uril Derivative. Org. Letters 2012, 14, 3072-3075.

(10) Huang, W.-H.; Liu, S.; Zavalij, P. Y.; Isaacs, L. Nor-Seco-Cucurbit[10]uril Exhibits Homotropic Allosterism. J. Am. Chem. Soc. 2006, 128, 14744-14745.

(11) Isaacs, L.; Park, S.-K.; Liu, S.; Ko, Y. H.; Selvapalam, N.; Kim, Y.; Kim, H.; Zavalij, P. Y.; Kim, G.-H.; Lee, H.-S.; Kim, K. The Inverted Cucurbit[n]uril Family. J. Am. Chem. Soc. 2005, 127, 18000-18001.

(12) Cintas, P. Cucurbituril: Supramolecular Perspectives for an Old Ligand. J. Incl. Phenom. Mol. Recognit. Chem. 1994, 17, 205-220.

(13) Gerasko, O. A.; G. Samsonenko, D. G.; Fedin, V. P. Supramolecular Chemistry of Cucurbiturils. Russ. Chem. Rev. 2002, 71, 741-760.

(14) Masson, E.; Ling, X.; Joseph, R.; Kyeremeh-Mensah, L.; Lu, X. Cucurbituril Chemistry: a Tale of Supramolecular Success. RSC Adv. 2012, 2, 1213-1247. 
(15) Lagona, J.; Mukhopadhyay, P.; Chakrabarti, S.; Isaacs, L. The Cucurbit[n]uril Family. Angew. Chem. Int. Ed. 2005, 44, 4844-4870.

(16) Lü, J.; Lin, J. X.; Cao, M. N.; Cao, R. Cucurbituril: A Promising organic Building Block for the Design of Coordination Compounds and Beyond. Coordin. Chem. Rev. 2013, 257, 1334-1356.

(17) Assaf, K. I.; Nau, W. M. Cucurbiturils: From Synthesis to High-Affinity Binding and Catalysis. Chem. Soc. Rev. 2015, 44, 394-418.

(18) Walker, S.; Oun, R.; McInnes, F. J.; Wheate, N. J. The Potential of Cucurbit[n]urils in Drug Delivery. Isr. J. Chem. 2011, 51, 616-624.

(19) Elbashir, A. A.; Aboul-Enein, H. Y. Critical Reviews in Analytical Chemistry Supramolecular Analytical Application of Cucurbit[n]urils Using Fluorescence Spectroscopy Supramolecular Analytical Application of Cucurbit[n]urils Using Fluorescence Spectroscopy. Crit. Rev. Anal. Chem. 2015, 45, 52-61.

(20) Pemberton, B. C.; Singh, R. K.; Johnson, A. C.; Jockusch, S.; Da Silva, J. P.; Ugrinov, A.; Turro, N. J.; Srivastava, D. K.; Sivaguru, J. Supramolecular Photocatalysis: Insights into Cucurbit[8]uril Catalyzed Photodimerization of 6-methylcoumarin. Chem. Commun. 2011, 47, 6323-6325.

(21) Li, H.; Yang, Y.-W. Gold Nanoparticles Functionalized with Supramolecular Macrocycles. Chin. Chem. Lett. 2013, 24, 545-552.

(22) Montes-García, V.; Pérez-Juste, J.; Pastoriza-Santos, I.; Liz-Marzán, L. M. Metal Nanoparticles and Supramolecular Macrocycles: a Tale of Synergy. Chem. Eur. J. 2014, 20, 10874-10883.

(23) Gürbüz, S.; Idris, M.; Tuncel, D. Cucurbituril-Based Supramolecular Engineered Nanostructured Materials. Org. Biomol. Chem. 2015, 13, 330-347. 
(24) Jeon, Y. M.; Kim, J.; Whang, D.; Kim, K. Molecular Container Assembly Capable of Conrolling Binding and Release of its Guest Molecules: Reversible Encapsulation of Organic Molecules in Sodium Ion Complexed Cucurbituril. J. Am. Chem. Soc. 1996, $118,9790-9791$.

(25) Whang, D.; Heo, J.; Park, J. H.; Kim, K. A Molecular Bowl with Metal Ion as Bottom: Reversible Inclusion of Organic Molecules in Cesium Ion Complexed Cucurbituril. Angew. Chem. Int. Ed. 1998, 37, 78-80.

(26) Gerasko, O. A.; Virovets, A. V.; Samsonenko, D. G.; Tripol"skaya, A. A.; Fedin, V. P.; Fenske, D. Synthesis and Crystal Structures of Supramolecular Sompounds of Sucurbit[n]urils $(n=6,8)$ with Polynuclear Strontium Aqua Complexes. Russ. Chem. Bull. 2003, 52, 585-593.

(27) Danylyuk, O.; Fedin, V. P. Solid-State Supramolecular Assemblies of Tryptophan and Tryptamine with Cucurbit[6]uril. Cryst. Growth Des. 2012, 12, 550-555.

(28) Liu, J. X.; Long, L.-S.; Huang, R.-B.; Zheng, L.-S. Molecular Capsules Based on Cucurbit[5]uril Encapsulating Naked Anion Chlorine. Cryst. Growth Des. 2006, 6, 26112614 .

(29) Buschmann, H. J.; Cleve, E.; Jansen, K.; Schollmeyer, E. Determination of Complex Stabilities with Nearly Insoluble Host Molecules: Cucurbit[5]uril, Decamethylcucurbit[5]uril and Cucurbit[6]uril as Ligands for the Complexation of some Multicharged Cations in Aqueous Solution. Anal. Chim. Acta 2001, 437, 157-163.

(30) Gerasko, O. A.; Mainicheva, E. A.; Naumova, M. I.; Neumaier, M.; Kappes, M. M.; Lebedkin, S.; Fenske, D.; Fedin, V. P. Sandwich-Type Tetranuclear Lanthanide Complexes with Cucurbit[6]uril: From Molecular Compounds to Coordination Polymers. Inorg. Chem. 2008, 47, 8869-8880. 
(31) Thuéry P, Uranyl Ion Complexes with Cucurbit[5]uril: from Molecular Capsules to Uranyl-Organic Frameworks. Cryst. Growth Des. 2009, 9, 1208-1215.

(32) Liu, J. X.; Long, L. S.; Huang, R. B.; Zheng, L. S. Interesting Anion-Inclusion Behavior of Cucurbit[5]uril and Its Lanthanide-Capped Molecular Capsule. Inorg. Chem. 2007, 46, 10168-10173.

(33) Samsonenko, D. G.; Lipkowski, J.; Gerasko, O. A.; Virovets, A. V.; Sokolov, M. N.; Fedin, V. P.; Platas, J. G.; Hernandez-Molina, R.; Mederos, A. Cucurbituril as a New Macrocyclic Ligand for Complexation of Lanthanide Cations in Aqueous Solutions. Eur. J. Inorg. Chem. 2002, 2002, 2380-2388.

(34) Jansen, K.; Buschmann, H. J.; Wego, A.; Döpp, D.; Mayer, C.; Drexler, H. J.; Holdt, H. J.; Schollmeyer, E. Cucurbit[5]uril, Decamethylcucurbit[5]uril and ucurbit[6]uril. Synthesis, Solubility and Amine Complex Formation. J. Inc. Phenom. Macro. 2001, 39, 357-363.

(35) Márquez, C.; Hudgins, R. R.; Nau, W. M. Mechanism of HostGuest Complexation by Cucurbituril. J. Am. Chem. Soc. 2004, 126, 5806-5816.

(36) Ni, X.-L.; Xiao, X.; Cong, H.; Liang, L.-L.; Cheng, K.; Cheng, X.-J.; Ji, N.-N.; Zhu, Q.J.; Xue, S.-F.; Tao, Z. Cucurbit[n]uril-Based Coordination Chemistry: from Simple Coordination Complexes to Novel Poly-dimensional Coordination Polymers. Chemical Society reviews 2013, 42, 9480-508.

(37) Liu, L.; Zhao, N.; Scherman, O. A. Ionic liquids as Novel Guests for Cucurbit[6]uril in Neutral Water. Chem. Commun. 2008, 1070-1072.

(38) Zhao, N.; Liu, L.; Biedermann, F.; Scherman, O. A. Binding Studies on CB[6] with a Series of 1-Alkyl-3-methylimidazolium Ionic Liquids in an Aqueous System. Chem. Eur. J. 2010, 5, 530-537. 
(39) Isaacs, L. Cucurbit[n]urils: from Mechanism to Structure and Function. Chem. Commun. 2009, 619-629.

(40) Mecozzi, S.; Rebek, J. The 55\% Solution: A Formula for Molecular Recognition in the Liquid State. Chem.-Eur. J. 1998, 4, 1016-1022.

(41) Nau, W. M.; Florea, M.; Assaf, K. I. Deep Inside Cucurbiturils: Physical Properties and Volumes of their Inner Cavity Determine the Hydrophobic Driving Force for Host-Guest Complexation. Isr. J. Chem. 2011, 51, 559-577.

(42) Biedermann, F.; Scherman, O. A. Cucurbit[8]uril Mediated Donor-Acceptor Ternary Complexes: A Model System for Studying Charge-Transfer Interactions. J. Phys. Chem. B 2012, 116, 2842-2849.

(43) Biedermann, F.; Uzunova, V. D.; Scherman, O. A.; Nau, W. M.; De Simone, A. Release of High-Energy Water as an Essential Driving Force for the High-Affinity Binding of Cucurbit[n]urils. J. Am. Chem. Soc. 2012, 134, 15318-15323.

(44) Biedermann, F.; Vendruscolo, M.; Scherman, O. A.; De Simone, A.; Nau, W. M. Cucurbit[8]uril and Blue-Box: High-Energy Water Release Overwhelms Electrostatic Interactions. J. Am. Chem. Soc. 2013, 135, 14879-88.

(45) Biedermann, F.; Nau, W. M.; Schneider, H.-J. The Hydrophobic Effect RevisitedStudies with Supramolecular Complexes Imply High-Energy Water as a Noncovalent Driving Force. Angew. Chem. Int. Ed. 2014, 53, 11158-11171.

(46) Buschmann, H. J.; Cleve, E.; Jansen, K.; Wego, A.; Schollmeyer, E. Complex Formation between Cucurbit[n]urils and Alkali, Alkaline Earth and Ammonium Ions in Aqueous Solution. J. Inc. Phenom. Macro. 2001, 40, 117-120.

(47) Shen, Y.; Xue, S.; Zhao, Y.; Zhu, Q.; Tao, Z. NMR Study on Self-Assembled Cage 
Complex of Hexamethylenetetramine and Cucurbit[n]urils. Chin. Sci. Bull. 2003, 48, 2694-2697.

(48) Buschmann, H. J.; Cleve, E.; Jansen, K.; Wego, A.; Schollmeyer, E. The Determination of Complex Stabilities Between Different Cyclodextrins and Dibenzo18-Crown-6, Cucurbit[6]uril, Decamethylcucurbit[5]uril, Cucurbit[5]uril, p-tertbutylcalix[4]arene and p-tert-butylcalix[6]arene in Aqueous Solutions using a Spectrophoto. Mater. Sci. Eng. C 2001, 14, 35-39.

(49) Kellersberger, K. A.; Anderson, J. D.; Ward, S. M.; Krakowiak, K. E.; Dearden, D. V. Encapsulation of N2, O2, Methanol, or Acetonitrile by Decamethylcucurbit[5]uril(NH4+)2 Complexes in the Gas Phase: Influence of the Guest on "Lid" Tightness. J. Am. Chem. Soc. 2001, 123, 11316-11317.

(50) Mock, W. L.; Shih, N. Y. Host-Guest Binding Capacity of Cucurbituril. J. Org. Chem. $1983,48,3618-3619$.

(51) Kolman, V.; Marek, R.; Strelcova, Z.; Kulhanek, P.; Necas, M.; Svec, J.; Sindelar, V. Electron Density Shift in Imidazolium Derivatives upon Complexation with Cucurbit[6]uril. Chem.-Eur. J. 2009, 15, 6926-6931.

(52) Jiao, D.; Scherman, O. A. Isolation of Cucurbit[n]uril Homologues with Imidazolium Salts in a Recyclable Manner. Green Chem. 2012, 14, 2445-2449.

(53) Lee, S. J. C.; Lee, J. W.; Lee, H. H.; Seo, J.; Noh, D. H.; Ko, Y. H.; Kim, K.; Kim, H. I. Host-guest Chemistry from Solution to the Gas Phase: An Essential Role of Direct Interaction with Water for High-affinity Binding of Cucurbit[n]urils. J. Phys. Chem. B 2013, 117, 8855-8864.

(54) Efremova, O. A.; Mironov, Y. V.; Kuratieva, N. V.; Fedorov, V. E. Novel Supramolecular Compounds Based on Cucurbit[6]uril, 1,8-diaminooctane and Octahedral 
Thiohydroxo Anions with Cluster Core [Re6S 8]. Inorg. Chim. Acta 2010, 363, 44114415.

(55) Rekharsky, M. V.; Yamamura, H.; Kawai, M.; Osaka, I. Sequential Formation of a Ternary Complex among Dihexylammonium , Cucurbit [6] uril and Cyclodextrin with Positive Cooperativity: A Combined Calorimetric, NMR and ESI-MS Study. Org. Lett 2006, 8, 815-818.

(56) Kolman, V.; Khan, M. S. A.; Babinský, M.; Marek, R.; Sindelar, V. Supramolecular Shuttle Based on Inclusion Complex Between Cucurbit[6]uril and Bispyridinium Ethylene. Org. Letters 2011, 13, 6148-6151.

(57) Sun, X.; Li, B.; Cao, J.; Chen, J.; Wang, N.; Wan, D.; Zhang, H.; Zhou, X. Pseudopolyrotaxanes of Cucurbit[6]uril: A Three-Dimensional Network Self-assembled by ClO4-(H2O)2 Water Clusters. Chin. J. Org. Chem. 2012, 30, 941-946.

(58) Sun, X.; Li, B.; Xia, C.; Zhou, X.; Zhang, H. "Liquid-like" Type (COO-)2(H2O)10 Anion Water Clusters in Three-Dimensional Supramolecular Structure of Cucurbit[6]uril. Cryst. Eng. Comm. 2012, 14, 8525-8529.

(59) Grigoras, M.; Catargiu, A.-M.; Timpu, D. Modification of Polyanilines Properties Using Macrocyclic Compounds. Rev. Roum. Chim. 2012, 57, 781-790.

(60) Yang, H.; Ma, Q.; Tan, Y. Side-Chain Polypseudorotaxanes by Threading Cucurbit[6]uril onto N'-3-vinylbenzyldiaminobutane Dihydrochloride: Synthesis, Characterization, and Properties. J. Polym. Res. 2013, 20, 2-8.

(61) Wang, H.; Zhu, Y.; Ren, X.; Wang, M.; Tan, Y. Synthesis and Properties of Block Polypseudorotaxanes by Threading Cucurbit[6]uril. Iran. Polym. J. 2012, 21, 783792. 
(62) He, S.; Zhou, C.; Zhang, H.; Zhou, X. Binding Modes of Cucurbit[6]uril and Cucurbit[7]uril with a Series of Bis-pyridinium Compounds. J. Inc. Phenom. Macro. 2013, $76,333-344$.

(63) Tuncel, D.; Cindir, N.; Koldemir, U. . Rotaxane and [5]pseudorotaxane Based on Cucurbit[6]uril and Anchored to a Meso-Tetraphenyl Porphyrin. J. Inc. Phenom. Macro. 2006, 55, 373-380.

(64) Pievo, R.; Casati, C.; Franchi, P.; Mezzina, E.; Bennati, M.; Lucarini, M. End-toEnd Distance Determination in a Cucurbit[6]uril-Based Rotaxane by PELDOR Spectroscopy. Chem. Phys. Chem. 2012, 13, 2659-2661.

(65) Tuncel, D.; Ozsar, O.; Tiftik, H. B.; Salih, B. Molecular Switch Based on a Cucurbit[6] uril Containing Bistable [3]rotaxane. Chem. Commun. 2007, 1369-1371.

(66) Hu, F.; Huang, J.; Cao, M.; Chen, Z.; Yang, Y.-W.; Liu, S. H.; Yin, J. Dithienylethenebased Rotaxanes: Synthesis, Characterization and Properties. Org. Biomol. Chem. 2014, 12, 7712-7720.

(67) Im Jun, S.; Wook Lee, J.; Sakamoto, S.; Yamaguchi, K.; Kim, K. Rotaxane-Based Molecular Switch with Fluorescence Signaling. Tetrahedron Lett. 2000, 41, 471-475.

(68) Praetorius, A.; Bailey, D. M.; Schwarzlose, T.; Nau, W. M. Design of a Fluorescent Dye for Indicator Displacement from Cucurbiturils: A Macrocycle-Responsive Fluorescent Switch Operating through a pKa Shift. Org. Lett. 2008, 10, 4089-4092.

(69) Florea, M.; Nau, W. M. Strong Binding of Hydrocarbons to Cucurbituril Probed by Fluorescent Dye Displacement: A Supramolecular Gas-Sensing Ensemble. Angew. Chemie - Int. Ed.Angew. Chem. Int. Ed. 2011, 50, 9338-9342.

(70) Carvalho, C. P.; Ferreira, R.; Da Silva, J. P.; Pischel, U. An Aminonaphthalimide- 
Putrescine Conjugate as Fluorescent Probe for Cucurbituril Host-Guest Complexes. Supramol. Chem. 2012, 25, 1-10.

(71) Sueldo Occello, V. N.; Veglia, A. V. Cucurbit[6]uril Nanocavity as an Enhanced Spectrofluorimetric Method for the Determination of Pyrene. Anal. Chim. Acta 2011, $689,97-102$.

(72) Tadini, M. C.; Balbino, M. A.; Eleoterio, I. C.; De Oliveira, L. S.; Dias, L. G.; JeanFrançois Demets, G.; De Oliveira, M. F. Developing Electrodes Chemically Modified with Cucurbit[6]uril to Detect 3,4-Methylenedioxymethamphetamine (MDMA) by Voltammetry. Electrochim. Acta 2014, 121, 188-193.

(73) Danylyuk, O.; Fedin, V. P.; Sashuk, V. Kinetic Trapping of the Host-guest Association Intermediate and its Transformation into a Thermodynamic Inclusion Complex. Chem. Commun. 2013, 49, 1859-1861.

(74) Danylyuk, O.; Fedin, V. P.; Sashuk, V. Host-Guest Complexes of Cucurbit[6]uril with Isoprenaline: the Effect of the Metal Ion on the Crystallization Pathway and Supramolecular Architecture. Cryst. Eng. Comm. 2013, 15, 7414-7418.

(75) Freeman, B. Y. W. A. Structures of the P-Xylylenediammonium Chloride and Calcium Hydrogensulfate Adducts of the Cavitand Cucurbituril. Acta Cryst. 1984, b40, 382-387.

(76) Huang, W.-H. H.; Zavalij, P. Y.; Isaacs, L. Cucurbit[6]uril P-xylylenediammonium Diiodide Deca-hydrate Inclusion Complex. Acta Crystallogr. Sect. E Struct. 2008, 64, o1321-o1322.

(77) Guo, G.; Tang, Q.; Huang, Y.; Tao, Z.; Xue, S.; Zhu, Q. Influences on Shift of pKa , Solubility and Antifungal Activity of Fuberidazole by Inclusion Complexation with Cucurbit[n]urils. Chin. J. Org. Chem. 2014, 34, 2317. 
(78) Zheng, H.; Li, Z.; Zhang, J.; Ma, J.; Zhou, Y.; Jia, Q. Preparation of Cucurbit[6]urilModified Polymer Monolithic Column for Microextraction of Nitroaromatics. RSC Adv. 2015, 5, 5850-5857.

(79) Wang, L.; Wang, X.; Qi, M.; Fu, R. Cucurbit[6]uril in Combination with Guanidinium Ionic Liquid as a New Type of Stationary Phase for Capillary Gas Chromatography. J. Chromatogr. A 2014, 1334, 112-117.

(80) El-Sheshtawy, H. S.; Bassil, B. S.; Assaf, K. I.; Kortz, U.; Nau, W. M. Halogen Bonding Inside a Molecular Container. J. Am. Chem. Soc. 2012, 134, 19935-19941.

(81) Reddy, K. R. K. K.; Cavallini, T. S.; Demets, G. J. F.; Silva, L. F. Bromine and Iodine-Cucurbit[6]uril Complexes: Preparation and Applications in Synthetic Organic Chemistry. New J. Chem. 2014, 38, 2262-2264.

(82) Ren, H.; Huang, Z.; Yang, H.; Xu, H.; Zhang, X. Controlling the Reactivity of the Se-Se Bond by the Supramolecular Chemistry of Cucurbituril. Chem. Phys. Chem. $2015,16,523-527$.

(83) Thuéry, P. Supramolecular Assemblies built from Lanthanide Ammoniocarboxylates and Cucurbit[6]uril. Cryst. Eng. Comm. 2012, 14, 8128-8136.

(84) Thuéry, P. Uranyl-Lanthanide Heterometallic Assemblies with 1,2ethanedisulfonate and Cucurbit[6]uril Ligands. Cryst. Eng. Comm. 2012, 14, 3363-3366.

(85) Thuéry, P. Lanthanide Ion Complexes with 2-, 3-, or 4-Sulfobenzoate and Cucurbit[6]uril. Cryst. Growth Des. 2012, 12, 1632-1640.

(86) Blanco, E.; Quintana, C.; Hernández, P. An Electrochemical Study of Cucurbit[6]uril-Cadmium(II) Interactions and the Effect of Electrolyte Cations and Guest Molecules. Anal. Lett. 2014, 48, 783-795. 
(87) Zhang, T.; Chen, W.-J.; Zhang, Y.-Q.; Tao, Z.; Zhu, Q.-J. Coordination and Supramolecular Assemblies Based on Interaction of Three Different Cucurbit[6] urils with Sr(II)Ion. Chin. J. Inorg. Chem. 2014, 30, 473-480.

(88) Ji, N. N. N.; Cheng, X. J. J.; Zhao, Y.; Liang, L. L. L.; Chen, K.; Xiao, X.; Zhang, Y. Q. Q.; Zhu, Q. J. J.; Xue, S. F. F.; Tao, Z. Hexachloroplatinate(IV) Anion Induced Cucurbituril Supramolecular Assembly with Linear Channels. Eur. J. Inorg. Chem. 2014, 1435-1438.

(89) da Silva, F. F.; de Oliveira, C. A. F.; Falcão, E. H. L.; Chojnacki, J.; Neves, J. L.; Alves, S. New Lanthanide-CB[6] Coordination Compounds: Relationships Between the Crystal Structure and Luminescent Properties. Dalton Trans. 2014, 43, 5435-5442.

(90) Ren, M.; Pinkowicz, D.; Yoon, M.; Kim, K.; Zheng, L.-M. M.; Breedlove, B. K.; Yamashita, M. Dy(III) Single-ion Magnet Showing Extreme Sensitivity to (De)hydration. Inorg. Chem. 2013, 52, 8342-8348.

(91) Chen, K.; Kang, Y. S.; Zhao, Y.; Yang, J. M.; Lu, Y.; Sun, W. Y. Cucurbit[6]uril-Based Supramolecular Assemblies: Possible Application in Radioactive Cesium Cation Capture. J. Am. Chem. Soc. 2014, 2-5.

(92) Karcher, S.; Kornmüller, A.; Jekel, M. Effects of Alkali and Alkaline-Earth Cations on the Removal of Reactive Dyes with Cucurbituril. Acta Hydrochim. Hydrobiol. 1999, $27,38-42$.

(93) Khan, M. S. a.; Heger, D.; Necas, M.; Sindelar, V. Remarkable Salt Effect on Stability of Supramolecular Complex Between Modified Cucurbit[6]uril and Methylviologen in Aqueous Media. J. Phys. Chem. B 2009, 113, 11054-11057.

(94) Liu, P.; Shao, X.; Chipot, C.; Cai, W. Complexation Mechanism of Cucurbit[6]uril with Hexamethylene Diammonium Cations in Saline Solution. Phys. Chem. Chem. Phys. 2014, 16, 24169-24172. 
(95) Lee, J. W.; Samal, S.; Selvapalam, N.; Kim, H.-J.; Kim, K. Cucurbituril Homologues and Derivatives: New Opportunities in Supramolecular Chemistry. Acc. Chem. Res. 2003, 36, 621-630.

(96) Chinai, J. M.; Taylor, A. B.; Ryno, L. M.; Hargreaves, N. D.; Morris, C. A.; Hart, P. J.; Urbach, A. R. Molecular Recognition of Insulin by a Synthetic Receptor. J. Am. Chem. Soc. 2011, 133, 8810-8813.

(97) Smith, L. C.; Leach, D. G.; Blaylock, B. E.; Ali, O. A.; Urbach, A. R. SequenceSpecific, Nanomolar Peptide Binding via Cucurbit[8]uril-Induced Folding and Inclusion of Neighboring Side Chains. J. Am. Chem. Soc. 2015, 137, 3663-3669.

(98) Kasera, S.; Herrmann, L. O.; del Barrio, J.; Baumberg, J. J.; Scherman, O. A. Quantitative Multiplexing with Nano-Self-Assemblies in SERS. Sci. Rep. 2014, 4.

(99) Uzunova, V. D.; Cullinane, C.; Brix, K.; Nau, W. M.; Day, A. I. Toxicity of Cucurbit[7]uril and Cucurbit[8]uril: an Exploratory in Vitro and in Vivo Study. Org. Biomol. Chem. 2010, 8, 2037-2042.

(100) Hettiarachchi, G.; Nguyen, D.; Wu, J.; Lucas, D.; Ma, D.; Isaacs, L.; Briken, V. Toxicology and Drug Delivery by Cucurbit[n]uril Type Molecular Containers. PLoS One 2010, 5, e10514.

(101) Oun, R.; Floriano, R. S.; Isaacs, L.; Rowan, E. G.; Wheate, N. J. The Ex Vivo Neurotoxic, Myotoxic and Cardiotoxic Activity of Cucurbituril-Based Macrocyclic Drug Delivery Vehicles. Toxicol. Res. 2014, 3, 447-455.

(102) Chen, H.; Chan, J. Y. W.; Yang, X.; Wyman, I. W.; Bardelang, D.; Macartney, D. H.; Lee, S. M. Y.; Wang, R. Developmental and Organ-Specific Toxicity of Cucurbit[7]uril: in vivo Study on Zebrafish Models. RSC Adv. 2015, 5, 30067-30074. 
(103) Ryan, S. T. J.; Del Barrio, J.; Ghosh, I.; Biedermann, F.; Lazar, A. I.; Lan, Y.; Coulston, R. J.; Nau, W. M.; Scherman, O. A. Efficient Host-Guest Energy Transfer in Polycationic Cyclophane-Perylene Diimide Complexes in Water. J. Am. Chem. Soc. 2014, 136, 9053-9060.

(104) Ahn, Y.; Jang, Y.; Selvapalam, N.; Yun, G.; Kim, K. Supramolecular Velcro for Reversible Underwater Adhesion. Angew. Chem. Int. Ed. 2013, 52, 3140-3144.

(105) Chen, H.; Ma, H.; Tan, Y. Synthesis of Linear Cucurbit[7]uril Pendent Copolymers through Radical Polymerization: Polymers with Ultra-high Binding Affinity. J. Polym. Sci. Pol. Chem. A 2015, 1-5.

(106) Liu, S.; Ruspic, C.; Mukhopadhyay, P.; Chakrabarti, S.; Zavalij, P. Y.; Isaacs, L. The Cucurbit[n]uril Family: Prime Components for Self-Sorting Systems. J. Am. Chem. Soc. 2005, 127, 15959-15967.

(107) Kim, S. Y.; Jung, I. S.; Lee, E.; Kim, J.; Sakamoto, S.; Yamaguchi, K.; Kim, K. Macrocycles within Macrocycles: Cyclen, Cyclam, and their Transition Metal Complexes Encapsulated in Cucurbit[8]uril. Angew. Chem. Int. Ed. 2001, 40, 2119-2121.

(108) Hart, S. L.; Haines, R. I.; Decken, A.; Wagner, B. D. Isolation of the Trans-I and Trans-II Isomers of $\mathrm{Cu}-\mathrm{II}(\mathrm{cyclam})$ via Complexation with the Macrocyclic Host $\mathrm{Cu}$ curbit[8]uril. Inorg. Chim. Acta 2009, 362, 4145-4151.

(109) Mitkina, T. V.; Naumov, D. Y.; Gerasko, O. A.; Dolgushin, F. M.; Vicent, C.; Llusar, R.; Sokolov, M. N.; Fedin, V. P. Inclusion of Nickel(II) and Copper(II) Complexes with Aliphatic Polyamines in Cucurbit[8]uril. Russ. Chem. Bull. 2004, 53, 2519-2524.

(110) Kovalenko, E. A.; Mitkina, T. V.; Gerasko, O. A.; Samsonenko, D. G.; Naumov, D. Y.; Fedin, V. P. Cucurbit[8]uril-Based Inclusion Compounds Containing Iron(II), Cobalt(III), and Nickel(II) Complexes with Cyclam and Cyclen as Guest 
Molecules: Synthesis and Crystal Structures. Russ. J. Coordin. Chem. 2011, 37, 161167.

(111) Mitkina, T. V.; Naumov, D. Y.; Gerasko, O. A.; Fedin, V. P. Crystal Structure and Chemical Oxidation of the Palladium(II) Cyclam Complex within the Cavity of $\mathrm{Cu}$ curbit[8]uril. Inorg. Chim. Acta 2010, 363, 4387-4391.

(112) Wu, X.-j.; Hu, K.; Meng, X. G.; Cheng, G. Z. A cucurbit[8]uril Inclusion Complex with 1,7-Dimethyl-1,4,7,10-Tetraazacyclododecane Tetrachloride. New J. Chem. 2010, 34, 17-20.

(113) Jiang, G.; Li, G. Preparation and Biological Activity of Novel Cucurbit[8]urilFullerene Complex. J. Photoch. Photobio. B 2006, 85, 223-227.

(114) Ko, Y. H.; Kim, H.; Kim, Y.; Kim, K. U-shaped Conformation of Alkyl Chains Bound to a Synthetic Host. Angew. Chem. Int. Ed. 2008, 47, 4106-4109.

(115) Ko, Y. H.; Kim, Y.; Kim, H.; Kim, K. U-Shaped Conformation of Alkyl Chains Bound to a Synthetic Receptor Cucurbit[8]uril. Chem. Asian J. 2011, 6, 652-657.

(116) Xiao, X.; Wang, Q.; Yu, Y. H.; Xiao, Z. Y.; Tao, Z.; Xue, S.-F.; Zhu, Q. J.; Liu, J. X.; Liu, X. H. Contorted Conformations of 1,4-Butylidenedipyridinium and 1,10Decylidenedipyridinium Cationic Guests in a Cucurbit[8]uril Host. Eur. J. Inorg. Chem. 2011, 2366-2371.

(117) Baek, K.; Kim, Y.; Kim, H.; Yoon, M.; Hwang, I.; Ko, Y. H.; Kim, K. Unconventional U-Shaped Conformation of a Bolaamphiphile Embedded in a Synthetic Host. Chem. Commun. 2010, 46, 4091-4093.

(118) Mileo, E.; Mezzina, E.; Grepioni, F.; Pedulli, G. F.; Lucarini, M. Preparation and Characterisation of a New Inclusion Compound of Cucurbit[8]uril with a Nitroxide Radical. Chem. Eur. J. 2009, 15, 7859-7862. 
(119) Bardelang, D.; Banaszak, K.; Karoui, H.; Rockenbauer, A.; Waite, M.; Udachin, K.; Ripmeester, J. A.; Ratcliffe, C. I.; Ouari, O.; Tordo, P. Probing Cucurbituril Assemblies in Water with TEMPO-like Nitroxides: A Trinitroxide Supraradical with SpinSpin Interactions. J. Am. Chem. Soc. 2009, 131, 5402-5404.

(120) Jayaraj, N.; Porel, M.; Ottaviani, M. F.; Maddipatla, M. V. S. N.; Modelli, A.; Da Silva, J. P.; Bhogala, B. R.; Captain, B.; Jockusch, S.; Turro, N. J.; Ramamurthy, V. Self Aggregation of Supramolecules of Nitroxides@Cucurbit[8]uril Revealed by EPR Spectra. Langmuir, 2009, 25, 13820-13832.

(121) Yi, S.; Captain, B.; Ottaviani, M. F.; Kaifer, A. E. Controlling the Extent of Spin Exchange Coupling in 2,2,6,6-Tetramethylpiperidine-1-oxyl (TEMPO) Biradicals via Molecular Recognition with Cucurbit[n]uril Hosts. Langmuir 2011, 27, 5624-5632.

(122) Yi, S.; Captain, B.; Kaifer, A. E. The Importance of Methylation in the Binding of (Ferrocenylmethyl)tempammonium Guests by Cucurbit[n]uril $(n=7,8)$ Hosts. Chem. Commun. 2011, 47, 5500-5502.

(123) Tootoonchi, M. H.; Yi, S.; Kaifer, A. E. Detection of Isomeric Microscopic Host-Guest Complexes. A Time-Evolving Cucurbit[7]uril Complex. J. Am. Chem. Soc. 2013, 135, 10804-10809.

(124) Bardelang, D.; Casano, G.; Poulhes, F.; Karoui, H.; Filippini, J.; Rockenbauer, A.; Rosas, R.; Monnier, V.; Siri, D.; Gaudel-Siri, A.; Ouari, O.; Tordo, P. Spin Exchange Monitoring of the Strong Positive Homotropic Allosteric Binding of a Tetraradical by a Synthetic Receptor in Water. J. Am. Chem. Soc. 2014, 136, 17570-17577.

(125) Porel, M.; Jockusch, S.; Ottaviani, M. F.; Turro, N. J.; Ramamurthy, V. Interaction between Encapsulated Excited Organic Molecules and Free Nitroxides: Communication Across a Molecular Wall. Langmuir 2011, 27, 10548-10555. 
(126) Rinkevicius, Z.; Frecu, B.; Murugan, N. A.; Vahtras, O.; Kongsted, J.; Agren, H. Encapsulation Influence on EPR Parameters of Spin-Labels: 2,2,6,6-Tetramethyl-4methoxypiperidine-1-oxyl in Cucurbit[8]uril. J. Chem. Theory Comput. 2012, 8, 257263.

(127) Peresypkina, E. V.; Fedin, V. P.; Maurel, V.; Grand, A.; Rey, P.; Vostrikova, K. E. Inclusion of a Nitronyl Nitroxyl Radical and Its Hydrochloride in Cucurbit[8]uril. Chem.-Eur. J. 2010, 16, 12481-12487.

(128) Kim, H.-J.; Heo, J.; Jeon, W. S.; Lee, E.; Kim, J.; Sakamoto, S.; Yamaguchi, K.; Kim, K. Selective Inclusion of a Hetero-Guest Pair in a Molecular Host: Formation of Stable Charge-Transfer Complexes in Cucurbit[8]uril. Angewandte Chemie 2001, 113, 15741577.

(129) Kim, H. J.; Kim, K.; Kim, S. Y.; Oh, J.; Zhao, J. Water- and Organic-Soluble Cucurbituril Derivatives, their Preparation Methods, their Separation Methods and uses. 2003.

(130) Bush, M. E.; Bouley, N. D.; Urbach, A. R. Charge-Mediated Recognition of NTerminal Tryptophan in Aqueous Solution by a Synthetic Host. J. Am. Chem. Soc. 2005, 127, 14511-14517.

(131) Wang, R.; Yuan, L.; Macartney, D. H. Cucurbit[7]uril Mediates the Stereoselective Hydrochloride in Aqueous Solution. J. Org. Chem. 2006, 1237-1239.

(132) Ling, Y.; Wang, W.; Kaifer, A. E. A New Cucurbit[8]uril-Based Fluorescent Receptor for Indole Derivatives. Chem. Commun. 2007, 610-612.

(133) Loh, X. J. Supramolecular Host-Guest Polymeric Materials for Biomedical Applications. Mater. Horiz. 2014, 1, 185-195. 
(134) Gürbuz, S.; Idris, M.; Tuncel, D. Cucurbituril-Based Supramolecular Engineered Nanostructured Materials. Org. Biomol. Chem. 2015, 13, 330-347.

(135) Yang, X.; Yu, H.; Wang, L.; Tong, R.; Akram, M.; Chen, Y.; Zhai, X. Self-Healing Polymer Materials Constructed by Macrocycle-Based Host-Guest Interactions. Soft Matter 2015, 11, 1242-1252.

(136) Yang, C.; Mori, T.; Origane, Y.; Ko, Y. H.; Selvapalam, N.; Kim, K.; Inoue, Y. Highly Stereoselective Photocyclodimerization of Alpha-Cyclodextrin-Appended Anthracene Mediated by Gamma-Cyclodextrin and Cucurbit[8]uril: A Dramatic Steric Effect Operating Outside the Binding Site. J. Am. Chem. Soc. 2008, 130, 8574.

(137) Lei, L.; Luo, L.; Wu, X. L.; Liao, G. H.; Wu, L. Z.; Tung, C. H. Cucurbit[8]urilMediated Photodimerization of Alkyl 2-Naphthoate in Aqueous Solution. Tetrahedron Lett. 2008, 49, 1502-1505.

(138) Gromov, S. P.; Vedernikov, A. I.; Kuz'Mina, L. G.; Kondratuk, D. V.; Sazonov, S. K.; Strelenko, Y. A.; Alfimov, M. V.; Howard, J. A. K. Photocontrolled Molecular Assembler Based on Cucurbit[8]uril: [2+2]-Autophotocycloaddition of Styryl Dyes in the Solid State and in Water. Eur. J. Inorg. Chem. 2010, 2587-2599.

(139) Kasera, S.; Biedermann, F.; Baumberg, J. J.; Scherman, O. A.; Mahajan, S. Quantitative SERS Using the Sequestration of Small Molecules Inside Precise Plasmonic Nanoconstructs. Nano Lett. 2012, 12, 5924-5928.

(140) Roldán, M. L.; Sanchez-Cortes, S.; García-Ramos, J. V.; Domingo, C. Cucurbit[8]urilStabilized Charge Transfer Complexes with Diquat Driven by pH: a SERS Study. Phys. Chem. Chem. Phys. 2012, 14, 4935-4941.

(141) Taylor, R. W.; Coulston, R. J.; Biedermann, F.; Mahajan, S.; Baumberg, J. J.; Scherman, O. A. In situ SERS Monitoring of Photochemistry within a Nanojunction Reactor. Nano Lett. 2013, 13, 5985-5990. 
(142) Jeon, W. S.; Kim, H. J.; Lee, C.; Kim, K. Control of the Stoichiometry in Host-Guest Complexation by Redox Chemistry of Guests: Inclusion of Methylviologen in Cucurbit[8]uril. Chem. Commun. 2002, 1828-1829.

(143) Biedermann, F.; Rauwald, U.; Zayed, J. M.; Scherman, O. A. A Supramolecular Route for Reversible Protein-Polymer Conjugation. Chem Sci. 2011, 2, 279-286.

(144) Uhlenheuer, D. A.; Young, J. F.; Nguyen, H. D.; Scheepstraa, M.; Brunsveld, L. Cucurbit[8]uril Induced Heterodimerization of Methylviologen and Naphthalene Functionalized Proteins. Chem. Commun. 2011, 47, 6798-6800.

(145) Gonzalez-Campo, A.; Brasch, M.; Uhlenheuer, D. A.; Gomez-Casado, A.; Yang, L.; Brunsveld, L.; Huskens, J.; Jonkheijm, P. Supramolecularly Oriented Immobilization of Proteins Using Cucurbit[8]uril. Langmuir 2012, 28, 16364-16371.

(146) Rauwald, U.; Biedermann, F.; Deroo, S.; Robinson, C. V.; Scherman, O. A. Correlating Solution Binding and ESI-MS Stabilities by Incorporating Solvation Effects in a Confined Cucurbit[8]uril System. J. Phys. Chem. B 2010, 114, 8606-8615.

(147) Sindelar, V.; Cejas, M. A.; Raymo, F. M.; Chen, W. Z.; Parker, S. E.; Kaifer, A. E. Supramolecular Assemby of 2,7-Dimethyldiazapyrenium and Cucurbit $\{[\} 8]$ uril: A New Fluorescent Host for Detection of Catechol and Dopamine. Chem. Eur. J. 2005, 11, 7054-7059.

(148) Biedermann, F.; Rauwald, U.; Cziferszky, M.; Williams, K. A.; Gann, L. D.; Guo, B. Y.; Urbach, A. R.; Bielawski, C. W.; Scherman, O. A. Benzobis(imidazolium)Cucurbit[8]uril Complexes for Binding and Sensing Aromatic Compounds in Aqueous Solution. Chem. Eur. J. 2010, 16, 13716-13722.

(149) Jiao, D.; Biedermann, F.; Scherman, O. A. Size Selective Supramolecular Cages from Aryl-Bisimidazolium Derivatives and Cucurbit[8]uril. Org. Lett. 2011, 13, 30443047. 
(150) Biedermann, F.; Elmalem, E.; Ghosh, I.; Nau, W. M.; Scherman, O. A. Strongly Fluorescent, Switchable Perylene Bis(diimide) Host-Guest Complexes with Cucurbit[8] uril In Water. Angew. Chem. Int. Ed. 2012, 51, 7739-7743.

(151) Biedermann, F.; Hathazi, D.; Nau, W. M. Associative Chemosensing by Fluorescent Macrocycle-Dye Complexes - a Versatile Enzyme Assay Platform Beyond Indicator Displacement. Chem. Commun. 2015, 51, 4977-4980.

(152) Wang, R.; Bardelang, D.; Waite, M.; Udachin, K. A.; Leek, D. M.; Yu, K.; Ratcliffe, C. I.; Ripmeester, J. A. Inclusion Complexes of Coumarin in Cucurbiturils. Org. Biomol. Chem. 2009, 7, 2435-2439.

(153) Wu, X.; Meng, X.; Cheng, G. A novel 1:2 Cucurbit[8]uril Inclusion Complex with N-phenylpiperazine Hydrochloride. J. Inc. Phenom. Macro. 2009, 64, 325-329.

(154) Wang, R.; Yuan, L.; Ihmels, H.; Macartney, D. H. Cucurbit[8]uril/Cucurbit[7]uril Controlled Off/On Fluorescence of the Acridizinium and 9-Aminoacridizinium Cations in Aqueous Solution. Chem. Eur. J. 2007, 13, 6468-6473.

(155) Shaikh, M.; Choudhury, S. D.; Mohanty, J.; Bhasikuttan, A. C.; Pal, H. Contrasting Guest Binding Interaction of Cucurbit[7-8]urils with Neutral Red Dye: Controlled Exchange of Multiple Guests. Phys. Chem. Chem. Phys. 2010, 12, 7050-7055.

(156) Jiao, D.; Biedermann, F.; Tian, F.; Scherman, O. A. A Systems Approach to Controlling Supramolecular Architecture and Emergent Solution Properties via Host-Guest Complexation in Water. J. Am. Chem. Soc. 2010, 132, 15734-15743.

(157) Miskolczy, Z.; Biczok, L. Sequential Inclusion of Two Berberine Cations in Cucurbit[8]uril Cavity: Kinetic and Thermodynamic Studies. Phys. Chem. Chem. Phys. 2014, 16, 20147-20156. 
(158) Sayed, M.; Biedermann, F.; Uzunova, V. D.; Assaf, K. I.; Bhasikuttan, A. C.; Pal, H.; Nau, W. M.; Mohanty, J. Triple Emission from p-DimethylaminobenzonitrileCucurbit[8]uril Triggers the Elusive Excimer Emission. Chem. Eur. J. 2015, 21, 691696.

(159) Senler, S.; Cui, L.; Broomes, A. M.; Smith, E. L.; Wilson, J. N.; Kaifer, A. E. New Guests for the Cucurbit[8]uril Host. Formation of G2H Ternary Complexes. J. Phys. Org. Chem. 2012, 25, 592-596.

(160) Zhang, Y.; Zhou, T.-Y.; Zhang, K.-D.; Dai, J.-L.; Zhu, Y.-Y.; Zhao, X. Encapsulation Enhanced Dimerization of a Series of 4-Aryl-N-Methylpyridinium Derivatives in Water: New Building Blocks for Self-Assembly in Aqueous Media. Chem. Eur. J. 2014, 9, 1530-1534.

(161) Vincil, G. A.; Urbach, A. R. Effects of the Number and Placement of Positive Charges on ViologenCucurbit[n]uril Interactions. Supramol. Chem. 2008, 20, 681-687.

(162) Biedermann, F.; Ross, I.; Scherman, O. A. Host-guest Accelerated Photodimerisation of Anthracene-Labeled Macromolecules in Water. Polym. Chem. 2014, 5, 53755382.

(163) Carvalho, C. P.; Dominguez, Z.; Da Silva, J. P.; Pischel, U. A Supramolecular Keypad Lock. Chem. Commun. 2015, 51, 2698-2701.

(164) Sonzini, S.; Ryan, S. T. J.; Scherman, O. A. Supramolecular Dimerisation of MiddleChain Phe Pentapeptides via CB[8] Host-Guest Homoternary Complex Formation. Chem. Commun. 2013, 49, 8779-8781.

(165) Heitmann, L. M.; Taylor, A. B.; Hart, P. J.; Urbach, A. R. Sequence-Specific Recognition and Cooperative Dimerization of N-Terminal Aromatic Peptides in Aqueous Solution by a Synthetic Host. J. Am. Chem. Soc. 2006, 128, 12574-12581. 
(166) Ong, W.; Gómez-Kaifer, M.; Kaifer, A. E. Cucurbit[7]uril: A Very Effective Host for Viologens and Their Cation Radicals. Org. Lett. 2002, 4, 1791-1794.

(167) Hwang, I.; Ziganshina, A. Y.; Ko, Y. H.; Yun, G.; Kim, K. A New Three-Way Supramolecular Switch based on Redox-controlled Interconversion of Hetero- and Homo-Guest-Pair Inclusion Inside a Host Molecule. Chem. Commun. 2009, 416-418.

(168) Li, J. R.; Kuppler, R. J.; Zhou, H. C. Selective Gas Adsorption and Separation in Metal-Organic Frameworks. Chem. Soc. Rev. 2009, 38, 1477-1504.

(169) Tian, J.; Ma, S.; Thallapally, P. K.; Fowler, D.; McGrail, B. P.; Atwood, J. L. Cucurbit[7]uril: an Amorphous Molecular Material for Highly Selective Carbon Dioxide Uptake. Chem. Commun. 2011, 47, 7626-7628.

(170) Kim, H.; Kim, Y.; Yoon, M.; Lim, S.; Park, S. M.; Seo, G.; Kim, K. Highly Selective Carbon Dioxide Sorption in an Organic Molecular Porous Material. J. Am. Chem. Soc. 2010, 132, 12200-12202.

(171) Miyahara, Y.; Abe, K.; Inazu, T. "Molecular" Molecular Sieves: Lid-Free Decamethylcucurbit[5]uril Absorbs and Desorbs Gases Selectively. Angew. Chem. Int. Ed. 2002, 41, 3020-3023.

(172) Tian, J.; Liu, J.; Liu, J.; Thallapally, P. K. Identification of Solid-State forms of Cucurbit[6]uril for Carbon Dioxide Capture. Cryst. Eng. Comm. 2013, 15, 1528-1531.

(173) Haouaj, M. E.; Ho Ko, Y.; Luhmer, M.; Kim, K.; Bartik, K. NMR Investigation of the Complexation of Neutral Guests by Cucurbituril. J. Chem. Soc. Perkin Trans. 2, 2001, 2104-2107.

(174) El Haouaj, M.; Luhmer, M.; Ko, Y. H.; Kim, K.; Bartik, K. NMR Study of the Reversible Complexation of Xenon by Cucurbituril. J. Chem. Soc. Perkin Trans. 2 2001, 804-807. 
(175) Fusaro, L.; Locci, E.; Lai, A.; Luhmer, M. NMR Study of the Reversible Trapping of SF6 by Cucurbit[6]uril in Aqueous Solution. J. Phys. Chem. B 2008, 112, 15014-15020.

(176) Assaf, K. I.; Nau, W. M. Cucurbiturils as Fluorophilic Receptors. Supramol. Chem. 2014, 26, 657-669.

(177) Kim, B. S.; Ko, Y. H.; Kim, Y.; Lee, H. J.; Selvapalam, N.; Lee, H. C.; Kim, K. Water Soluble Cucurbit[6]uril Derivative as a Potential Xe Carrier for 129Xe NMR-Based Biosensors. Chem. Commun. 2008, 2756-2758.

(178) An, Q.; Li, G.; Tao, C.; Li, Y.; Wu, Y.; Zhang, W. A General and Efficient Method to form Self-Assembled Cucurbit[n]uril Monolayers on Gold Surfaces. Chem. Commun. 2008, 1989-1991.

(179) Premkumar, T.; Geckeler, K. E. Nanosized CuO Particles via a Supramolecular Strategy. Small 2006, 2, 616-620.

(180) Premkumar, T.; Lee, Y.; Geckeler, K. E. Macrocycles as a Tool: a Facile and One-Pot Synthesis of Silver Nanoparticles using Cucurbituril Designed for Cancer Therapeutics. Chem. - A Eur. J. 2010, 16, 11563-11566.

(181) Premkumar, T.; Geckeler, K. E. Cucurbit[7]uril as a Tool in the Green Synthesis of Gold Nanoparticles. Chem. Asian J. 2010, 5, 2468-2476.

(182) Lee, T. C.; Scherman, O. A. Formation of Dynamic Aggregates in Water by Cucurbit[5] uril Capped with Gold Nanoparticles. Chem. Commun. 2010, 46, 2438-2440.

(183) Taylor, R. W.; Lee, T. C.; Scherman, O. A.; Esteban, R.; Aizpurua, J.; Huang, F. M.; Baumberg, J. J.; Mahajan, S. Precise Subnanometer Plasmonic Junctions for SERS within Gold Nanoparticle Assemblies Using Cucurbit[n]uril "Glue". ACS Nano 2011, 5, 3878-3887. 
(184) Lee, T. C.; Scherman, O. A. A Facile Synthesis of Dynamic Supramolecular Aggregates of Cucurbit[n]uril ( $n=5-8)$ Capped with Gold Nanoparticles in Aqueous Media. Chem. Eur. J. 2012, 18, 1628-1633.

(185) Lanterna, A.; Pino, E. Enhanced Catalytic Electrochemical Reduction of Dissolved Oxygen with Ultraclean Cucurbituril[7]-Capped Gold Nanoparticles. Nanoscale 2014, 2, 9550-9553.

(186) Lu, X.; Masson, E. Formation and Stabilization of Silver Nanoparticles with Cucurbit[n]urils ( $n=5-8$ ) and Cucurbituril-Based Pseudorotaxanes in Aqueous Medium. Langmuir 2011, 3051-3058.

(187) Romero, I.; Aizpurua, J.; Bryant, G. W.; Abajo, F. J. G. D. Plasmons in Nearly Touching Metallic Nanoparticles: Singular Response in the Limit of Touching Dimers. Opt. Express 2006, 14, 9988-9999.

(188) Prodan, E.; Radloff, C.; Halas, N. J.; Nordlander, P. A Hybridization Model for the Plasmon Response of Complex Nanostructures. Science 2003, 302, 419-22.

(189) Esteban, R.; Borisov, A. G.; Nordlander, P.; Aizpurua, J. Bridging Quantum and Classical Plasmonics with a Quantum-Corrected Model. Nat. Commun. 2012, 3, 825.

(190) Scholl, J. A.; García-Etxarri, A.; Koh, A. L.; Dionne, J. A. Observation of Quantum Tunneling between Two Plasmonic Nanoparticles. Nano Letters 2013, 13, 564-569.

(191) Savage, K. J.; Hawkeye, M. M.; Esteban, R.; Borisov, A. G.; Aizpurua, J.; Baumberg, J. J. Revealing the Quantum Regime in Tunnelling Plasmonics. Nature 2012, $491,574-577$.

(192) Zhu, W.; Crozier, K. B. Quantum Mechanical Limit to Plasmonic Enhancement as Observed by Surface-Enhanced Raman Scattering. Nat. Commun. 2014, 5, 5228. 
(193) Esteban, R.; Zugarramurdi, A.; Zhang, P.; Nordlander, P.; Garcia-Vidal, F. J.; Borisov, A. G.; Aizpurua, J. A Classical Treatment of Optical Tunneling in Plasmonic Gaps: Extending the Quantum Corrected Model to Practical Situations. Faraday Discuss. 2015, 151-183.

(194) Esteban, R.; Taylor, R. W.; Baumberg, J. J.; Aizpurua, J. How Chain Plasmons Govern the Optical Response in Strongly Interacting Self-Assembled Metallic Clusters of Nanoparticles. Langmuir 2012, 28, 8881-8890.

(195) Herrmann, L. O.; Valev, V. K.; Aizpurua, J.; Baumberg, J. J. Self-sifting of Chain Plasmons: the Complex Optics of Au Nanoparticle Clusters. Opt. Express 2013, 21, 32377-32385.

(196) de Nijs, B.; Bowman, R. W.; Herrmann, L. O.; Benz, F.; Barrow, S. J.; Mertens, J.; Sigle, D. O.; Chikkaraddy, R.; Eiden, A.; Ferrari, A.; Scherman, O. A.; Baumberg, J. J. Unfolding the Contents of Sub-nm Plasmonic Gaps using Normalising Plasmon Resonance Spectroscopy. Faraday Discuss. 2015, 185-193.

(197) Herrmann, L. O.; Valev, V. K.; Tserkezis, C.; Barnard, J. S.; Kasera, S.; Scherman, O. A.; Aizpurua, J.; Baumberg, J. J. Threading Plasmonic Nanoparticle Strings with Light. Nat. Commun. 2014, 5, 4568.

(198) Hüsken, N.; Taylor, R. W.; Zigah, D.; Taveau, J. C.; Lambert, O.; Scherman, O. A.; Baumberg, J. J.; Kuhn, A. Electrokinetic Assembly of One-Dimensional Nanoparticle Chains with Cucurbit[7]uril Controlled Subnanometer Junctions. Nano Lett. $2013,13,6016-6022$.

(199) Jones, S. T.; Taylor, R. W.; Esteban, R.; Abo-Hamed, E. K.; Bomans, P. H. H.; Sommerdijk, N. a. J. M.; Aizpurua, J.; Baumberg, J. J.; Scherman, O. A. Gold Nanorods with Sub-nanometer Separation using Cucurbit[n]uril for SERS Applications. Small 2014, 10, 4298-4303. 
(200) Tao, C.-A.; An, Q.; Zhu, W.; Yang, H.; Li, W.; Lin, C.; Xu, D.; Li, G. Cucurbit[n]urils as a SERS Hot-Spot Nanocontainer through Bridging Gold Nanoparticles. Chem. Commun. 2011, 47, 9867-9869.

(201) Le Ru, E.; Etchegoin, P. Principles of Surface-Enhanced Raman Spectroscopy: and Related Plasmonic Effects, 1st ed.; Elsevier Science: Great Britain, 2008.

(202) Bell, S. E. J.; Sirimuthu, N. M. S. Quantitative Surface-Enhanced Raman Spectroscopy. Chem. Soc. Rev. 2008, 37, 1012-1024.

(203) Alvarez-Puebla, R. A.; Liz-Marzán, L. M. Traps and Cages for Universal SERS Detection. Chem. Soc. Rev. 2012, 41, 43-51.

(204) Guerrini, L.; Graham, D. Molecularly-Mediated Assemblies of Plasmonic Nanoparticles for Surface-Enhanced Raman Spectroscopy Applications. Chem. Soc. Rev. 2012, $41,7085-7107$.

(205) Corma, A.; Garcia, H.; Montes-Navajas, P. A Pseudopolyrotaxane Consisting in PPV Threaded in Multiple Cucurbiturils. Tetrahedron Lett. 2007, 48, 4613-4617.

(206) Mahajan, S.; Lee, T.-C.; Biedermann, F.; Hugall, J. T.; Baumberg, J. J.; Scherman, O. A. Raman and SERS Spectroscopy of Cucurbit[n]urils. Phys. Chem. Chem. Phys. 2010, 12, 10429-10433.

(207) Mohanty, J.; Nau, W. M. Ultrastable Rhodamine with Cucurbituril. Angew. Chem. Int. Ed. 2005, 44, 3750-3754.

(208) El-Barghouthi, M. I.; Assaf, K. I.; Rawashdeh, A. M. M. Molecular Dynamics of Methyl Viologen-Cucurbit[n]uril. J. Chem. Theory Comput. 2010, 984-992.

(209) Chen, Y.; Klimczak, A.; Galoppini, E.; Lockard, J. V. Structural Interrogation of a Cucurbit[7]uril-Ferrocene HostGuest Complex in the Solid State: a Raman Spectroscopy Study. RSC Adv. 2013, 3, 1354-1358. 
(210) Young, J. F.; Nguyen, H. D.; Yang, L.; Huskens, J.; Jonkheijm, P.; Brunsveld, L. Strong and Reversible Monovalent Supramolecular Protein Immobilization. Chem. Bio. Chem. 2010, 11, 180-183.

(211) Wasserberg, D.; Uhlenheuer, D. a.; Neirynck, P.; Cabanas-Danés, J.; Schenkel, J. H.; Ravoo, B. J.; An, Q.; Huskens, J.; Milroy, L. G.; Brunsveld, L.; Jonkheijm, P. Immobilization of Ferrocene-Modified SNAP-Fusion Proteins. Int. J. Mol. Sci. 2013, 14, 4066-4080.

(212) Neirynck, P.; Brinkmann, J.; An, Q.; van der Schaft, D. W. J.; Milroy, L.G.; Jonkheijm, P.; Brunsveld, L. Supramolecular Control of Cell Adhesion via Ferrocene-Cucurbit[7]uril Host-Guest Binding on Bold Surfaces. Chem. Commun. 2013, 49, 3679-3681.

(213) Freitag, M.; Galoppini, E. Cucurbituril Complexes of Viologens Bound to TiO2 Films. Langmuir 2010, 26, 8262-8269.

(214) Kim, K. K.; Jeon, W. S.; Kang, J.-K.; Lee, J. W.; Jon, S. Y.; Kim, T. A Pseudorotaxane on Gold: Formation of Self-Assembled Monolayers, Reversible Dethreading and Rethreading of the Ring, and Ion-Gating Behavior. Angew. Chem. Int. Ed. 2003, 42, 2395-2398.

(215) Ma, X.; Xue, Y.; Dai, L.; Urbas, A.; Li, Q. Hydrophilic Cucurbit[7]urilPseudorotaxane-Anchored-Monolayer-Protected Gold Nanorods. Eur. J. Inorg. Chem. 2013, 2013, 2682-2686.

(216) Halterman, R. L.; Moore, J. L.; Yip, W. T. Cucurbit[7]uril Disrupts Aggregate Formation Between Rhodamine B Dyes Covalently Attached to Glass Substrates. J. Fluoresc. 2011, 21, 1467-1478.

(217) Tang, Y.; Yang, S.; Zhao, Y.; You, M.; Zhang, F.; He, P. The Host - Guest Interaction 
Between Cucurbit[7]uril and Ferrocenemonocarboxylic Acid for Electrochemically Catalytic Determination of Glucose. Electroanalysis 2015,

(218) Yang, H.; An, Q.; Zhu, W.; Li, W.; Jiang, Y.; Cui, J.; Zhang, X.; Li, G. A New Strategy for Effective Construction of Protein Stacks by using Cucurbit[8]uril as a Glue Molecule. Chem. Commun. 2012, 48, 10633.

(219) Yeh, Y.-C.; Rana, S.; Mout, R.; Yan, B.; Alfonso, F. S.; Rotello, V. M. Supramolecular Tailoring of Protein-nanoparticle Interactions using Cucurbituril Mediators. Chem. Commun. 2014, 50, 5565-5568.

(220) Tian, F.; Cheng, N.; Nouvel, N.; Geng, J.; Scherman, O. A. Site-Selective Immobilization of Colloids on Au Substrates via a NoncovalentSupramolecular "Handcuff". Langmuir 2010, 26, 5323-5328.

(221) Tian, F.; Jiao, D.; Biedermann, F.; Scherman, O. a. Orthogonal Switching of a Single Supramolecular Complex. Nat. Commun. 2012, 3, 1207.

(222) Hu, C.; Lan, Y.; Tian, F.; West, K. R.; Scherman, O. A. Facile Method for Preparing Surface-Mounted Cucurbit[8]uril-Based Rotaxanes. Langmuir 2014, 30, 1092610932.

(223) Jones, S. T.; Zayed, J. M.; Scherman, O. A. Supramolecular Alignment of Gold Nanorods via Cucurbit[8]uril Ternary Complex Formation. Nanoscale 2013, 5, 52995302.

(224) Fraire, J. C.; Sueldo Occello, V.; Allende, L. G.; Veglia, A. V.; Coronado, E. A. Towards the Design of Highly Stable Small Colloidal SERS Substrates with Supramolecular Host-Guest Interactions for Ultrasensitive Detection. J. Phys. Chem. C 2015, 8876-8888. 
(225) Wei, L.; Wang, X.; Li, C.; Li, X.; Yin, Y.; Li, G. Colorimetric Assay for Protein Detection Based on Nano-Pumpkin Induced Aggregation of Peptide-Decorated Gold Nanoparticles. Biosens. Bioelectron. 2015, 71, 348-352.

(226) Lan, Y.; Wu, Y.; Karas, A.; Scherman, O. A. Photoresponsive Hybrid RaspberryLike Colloids Based on Cucurbit[8]uril Host-Guest Interactions. Angew. Chem. Int. Ed. 2014, 53, 2166-2169.

(227) Alberti, S.; Soler-Illia, G. J. A. A.; Azzaroni, O. Gated Supramolecular Chemistry in Hybrid Mesoporous Silica Nanoarchitectures: Controlled Delivery and Molecular Transport inRresponse to Chemical, Physical and Biological Stimuli. Chem. Commun. 2015, 6050-6075.

(228) Yang, Y. W.; Sun, Y. L.; Song, N. Switchable Host-Guest Systems on Surfaces. Acc. Chem. Res. 2014, 47, 1950-1960.

(229) Ambrogio, M. W.; Thomas, C. R.; Zhao, Y.-L. L.; Zink, J. I.; Stoddart, J. F. Mechanized silica Nanoparticles: A New Frontier in Theranostic Nanomedicine. Acc. Chem. Res. 2011, 44, 903-913.

(230) Klichko, Y.; Khashab, N. M.; Yang, Y. W.; Angelos, S.; Stoddart, J. F.; Zink, J. I. Improving Pore Exposure in Mesoporous Silica Films for Mechanized Control of the Pores. Microporous Mesoporous Mater. 2010, 132, 435-441.

(231) Angelos, S.; Yang, Y.-W. W.; Patel, K.; Stoddart, J. F.; Zink, J. I. pH-Responsive Supramolecular Nanovalves Based on Cucurbit[6]uril Pseudorotaxanes. Angew. Chem. Int. Ed. 2008, 47, 2222-2226.

(232) Angelos, S.; Khashab, N. M.; Yang, Y.-W. W.; Trabolsi, A.; Khatib, H. A.; Stoddart, J. F.; Zink, J. I. pH Clock-Operated Mechanized Nanoparticles. J. Am. Chem. Soc. 2009, 131, 12912-12914. 
(233) Angelos, S.; Yang, Y. W.; Khashab, N. M.; Stoddart, J. F.; Zink, J. I. Dual-Controlled Nanoparticles Exhibiting AND Logic. J. Am. Chem. Soc. 2009, 131, 11344-11346.

(234) Thomas, C. R.; Ferris, D. P.; Lee, J. H. H.; Choi, E.; Cho, M. H.; Kim, E. S.; Stoddart, J. F.; Shin, J. S. S.; Cheon, J.; Zink, J. I. Noninvasive Remote-Controlled Release of Drug Molecules in Vitro using Magnetic Actuation of Mechanized Nanoparticles. J. Am. Chem. Soc. 2010, 132, 10623-10625.

(235) Liu, J.; Du, X.pH- and Competitor-Driven Nanovalves of Cucurbit[7]uril Pseudorotaxanes Based on Mesoporous Silica Supports for Controlled Release. J. Mater. Chem. 2010, 20, 3642-3649.

(236) Liu, J.; Du, X.; Zhang, X. Enzyme-inspired Controlled Release of Cucurbit[7]uril Nanovalves by using Magnetic Mesoporous Silica. Chem. - A Eur. J. 2011, 17, 810815.

(237) Ambrogio, M. W.; Pecorelli, T. A.; Patel, K.; Khashab, N. M.; Trabolsi, A.; Khatib, H. A.; Botros, Y. Y.; Zink, J. I.; Stoddart, J. F. Snap-Top Nanocarriers. Org. Lett. 2010, 12, 3304-3307.

(238) Sun, Y.-1. L.; Yang, B. J. J.; Zhang, S. X. A. A.; Yang, Y. W. W. Cucurbit[7]uril Pseudorotaxane-Based Photoresponsive Supramolecular Nanovalve. Chem. Eur. J. 2012, 18, 9212-9216.

(239) Hwang, I.; Baek, K.; Jung, M.; Kim, Y.; Park, K. M.; Lee, D.-W.; Selvapalam, N.; Kim, K. Noncovalent Immobilization of Proteins on a Solid Surface by Cucurbit[7]uril-Ferrocenemethylammonium Pair, a Potential Replacement of Biotin-Avidin Pair. J. Am. Chem. Soc. 2007, 129, 4170-4171.

(240) Cui, S.-C.; Tachikawa, T.; Fujitsuka, M.; Majima, T. Photoinduced Electron Transfer in a Quantum DotCucurbituril Supramolecular Complex. J. Phys. Chem. C 2011, 115, 1824-1830. 
(241) Kimoon, K.; Young-Ho, K.; Kyeng-Min, P.; Narayanan, S.; Erumaipatty, R. N. Stationary Phase and Column Using Cucurbituril Bonded Silica Gel, and Separation Method of Taxol Using the Column. patent 20,080,290,028, 2008.

(242) Liu, S. M. M.; Xu, L.; Wu, C. T. T.; Feng, Y. Q. Q. Preparation and Characterization of Perhydroxyl-Cucurbit[6]uril Bonded Silica Stationary Phase for HydrophilicInteraction Chromatography. Talanta 2004, 64, 929-934.

(243) Li, L. S.; Wang, S. W.; Chen, X. Q.; Liu, C.; Xu, L.-L. Studies on Chromatographic Properties of Perhydroxycucurbit[6]uril as a New Type of Gas Chromatographic Column Packing Material. Chin. J. Chem. 2008, 26, 307-314.

(244) Nagarajan, E. R.; Oh, D. H.; Selvapalam, N.; Ko, Y. H.; Park, K. M.; Kim, K. Cucurbituril Anchored Silica Gel. Tetrahedron Lett. 2006, 47, 2073-2075.

(245) Miyake, J.; Chujo, Y. Molecular Recognizable Cucurbituril/Silica Hybrids. Chem. Lett. 2008, 37, 312-313.

(246) Zhang, P.; Qin, S.; Qi, M.; Fu, R. Cucurbit[n]urils as a New Class of Stationary Phases for Gas Chromatographic Separations. J. Chromatogr. A 2014, 1334, 139-148.

(247) Ma, L.; Liu, S.-M.; Yao, L.; Xu, L. Preparation and Chromatographic Performance Evaluation of Cucurbit[7]uril Immobilized Silica. J. Chromatogr. A 2015, 1376, 64-73.

(248) Zhu, X.; Fan, X.; Ju, G.; Cheng, M.; An, Q.; Nie, J.; Shi, F. A Facile Method to Immobilize Cucurbituril on Surfaces through Photocrosslinking with Azido Groups. Chem. Commun. 2013, 49, 8093-8095.

(249) del Pozo, M.; Hernandez, P.; Hernandez, L.; Quintana, C. The Use of Cucurbit[8]uril Host-Guest Interactions in the Development of an Electrochemical Sensor: Characterization and Application to Tryptophan Determination. J. Mater. Chem. 2011, 21, 13657-13663. 
(250) Kim, J.; Kim, Y.; Baek, K.; Ko, Y. H.; Kim, D.; Kim, K. Direct Force Measurement Between Cucurbit[6]uril and Spermine using Atomic Force Microscopy. Tetrahedron 2008, 64, 8389-8393.

(251) Gomez-Casado, A.; Jonkheijm, P.; Huskens, J. Recognition Properties of Cucurbit[7]uril Self-Assembled Monolayers Studied with Force Spectroscopy. Langmuir 2011, 27, 11508-11513.

(252) Blanco, E.; Quintana, C.; Hernández, L.; Hernández, P. Atomic Force Microscopy Study of new Sensing Platforms: Cucurbit[n]uril $(\mathrm{n}=6,7)$ on Gold. Electroanalysis 2013, 25, 263-268.

(253) Liu, Y.; Yang, H.; Wang, Z.; Zhang, X. Cucurbit[8]uril-Based Supramolecular Polymers. Chem. ASIAN J. 2013, 8, 1626-1632.

(254) Dong, S.; Zheng, B.; Wang, F.; Huang, F. Supramolecular Polymers Constructed from Macrocycle-Based Host-Guest Molecular Recognition Motifs. Acc. Chem. Res. 2014, 47, 1982-1994.

(255) Hu, J.; Liu, S. Engineering Responsive Polymer Building Blocks with Host-Guest Molecular Recognition for Functional Applications. Acc. Chem. Res. 2014, 47, 20842095.

(256) Li, H.; Wu, L. Metallo/clusto Hybridized Supramolecular Polymers. Soft Matter 2014, 10, 9038-9053.

(257) Yan, X.; Wang, F.; Zheng, B.; Huang, F. Stimuli-Responsive Supramolecular Polymeric Materials. Chem. Soc. Rev. 2012, 41, 6042-6065.

(258) Guo, D. S.; Liu, Y. Calixarene-Based Supramolecular Polymerization in Solution. Chem. Soc. Rev. 2012, 41, 5907-5921. 
(259) Sijbesma, R. P.; Beijer, F. H.; Brunsveld, L.; Folmer, B. J. B.; Hirschberg, J.; Lange, R. F. M.; Lowe, J. K. L.; Meijer, E. W. Reversible Polymers Formed from SelfComplementary Monomers using Quadruple Hydrogen Bonding. Science 1997, 278, 1601-1604.

(260) Lehn, J. M. Supramolecular Polymer Chemistry- Scope and Perspectives. Polym. Int. 2002, 51, 825-839.

(261) Yang, S. K.; Ambade, A. V.; Weck, M. Main-chain Supramolecular Block Copolymers. Chem. Soc. Rev. 2011, 40, 129-137.

(262) Brunsveld, L.; Folmer, B. J. B.; Meijer, E. W.; Sijbesma, R. P. Supramolecular Polymers. Chem. Rev. 2001, 101, 4071-4097.

(263) Wittmer, J. P.; van der Schoot, P.; Milchev, A.; Barrat, J. L. Dynamical Monte Carlo Study of Equilibrium Polymers. II. The Role of Rings. J. Chem. Phys. 2000, 113, 69927005 .

(264) Vermonden, T.; van der Gucht, J.; de Waard, P.; Marcelis, A. T. M.; Besseling, N. A. M.; Sudholter, E. J. R.; Fleer, G. J.; Stuart, M. A. C. Water-Soluble Reversible Coordination Polymers: Chains and Rings. Macromolecules 2003, 36, 7035-7044.

(265) Kricheldorf, H. R. The Role of Ring-Ring Equilibria in Thermodynamically Controlled Polycondensations. Macromol. Symp. 2003, 199, 15-22.

(266) Fox, J. D.; Rowan, S. J. Supramolecular Polymerizations and Main-Chain Supramolecular Polymers. Macromolecules 2009, 42, 6823-6835.

(267) Liu, S. M.; Zavalij, P. Y.; Isaacs, L. Cucurbit[10]uril. J. Am. Chem. Soc. 2005, 127, 16798-16799.

(268) Nally, R.; Isaacs, L. Toward Supramolecular Polymers Incorporating Double Cavity Cucurbituril Hosts. Tetrahedron 2009, 65, 7249-7258. 
(269) Lee, J. W.; Kim, K.; Choi, S.; Ko, Y. H.; Sakamoto, S.; Yamaguchi, K.; Kim, K. Unprecedented Host-Induced Intramolecular Charge-transfer Complex Formation. Chem. Commun. 2002, 2692-2693.

(270) Ko, Y. H.; Kim, K.; Kim, E.; Kim, K. Exclusive Formation of 1:1 and 2:2 Complexes Between Cucurbit[8]uril and Electron Donor-Acceptor Molecules Induced by HostStabilized Charge-Transfer Interactions. Supramol. Chem. 2007, 19, 287-293.

(271) Kim, K.; Kim, D.; Lee, J. W.; Ko, Y. H.; Kim, K. Growth of Poly(Pseudorotaxane) on Gold Using Host-Stabilized Charge-Transfer Interaction. Chem. Commun. 2004, 848-849.

(272) Ko, Y. H.; Kim, K.; Kang, J. K.; Chun, H.; Lee, J. W.; Sakamoto, S.; Yamaguchi, K.; Fettinger, J. C.; Kim, K. Designed Self-Assembly of Molecular Necklaces using HostStabilized Charge-Transfer Interactions. J. Am. Chem. Soc. 2004, 126, 1932-1933.

(273) Liu, Y.; Yu, Y.; Gao, J.; Wang, Z.; Zhang, X. Water-Soluble Supramolecular Polymerization Driven by Multiple Host-Stabilized Charge-Transfer Interactions. Angew. Chem. Int. Ed. 2010, 49, 6576-6579.

(274) Wang, L. X.; Bloomfilde, V. A. Small-Angle X-Ray Scattering of Semidilute Rodlike DNA Solutions- Polyelectrolyte Behavior. Macromolecules 1991, 24, 5791-5795.

(275) Muroga, Y.; Sakuragi, I.; Noda, I.; Nagawasa, M. Effect of Beta-Substitution on Chain Flexibility. Macromolecules 1984, 17, 1844-1847.

(276) del Barrio, J.; Horton, P. N.; Lairez, D.; Lloyd, G. O.; Toprakcioglu, C.; Scherman, O. A. Photocontrol over Cucurbit[8]uril Complexes: Stoichiometry and Supramolecular Polymers. J. Am. Chem. Soc. 2013, 135, 11760-11763.

(277) Liu, Y.; Liu, K.; Wang, Z.; Zhang, X. Host-Enhanced pi-pi Interaction for WaterSoluble Supramolecular Polymerization. Chem. Eur. J. 2011, 17, 9930-9935. 
(278) Liu, Y.; Fang, R.; Tan, X.; Wang, Z.; Zhang, X. Supramolecular Polymerization at Low Monomer Concentrations: Enhancing Intermolecular Interactions and Suppressing Cyclization by Rational Molecular Design. Chem. Eur. J. 2012, 18, 1565015654 .

(279) Tan, X.; Yang, L.; Liu, Y.; Huang, Z.; Yang, H.; Wang, Z.; Zhang, X. Water-Soluble Supramolecular Polymers Fabricated through Specific Interactions Between Cucurbit[8]uril and a Tripeptide of Phe-Gly-Gly. Polym. Chem. 2013, 4, 5378-5381.

(280) Yang, H.; Liu, Y.; Liu, K.; Yang, L.; Wang, Z.; Zhang, X. Rational Adjustment of Multicolor Emissions by Cucurbiturils-Based Host-Guest Chemistry and Photochemistry. Langmuir 2013, 29, 12909-12914.

(281) Ramaekers, M.; Wijnands, S. P. W.; van Dongen, J. L. J.; Brunsveld, L.; Dankers, P. Y. W. Cucurbit[8]uril Templated Supramolecular Ring Structure Formation and Protein Assembly Modulation. Chem. Commun. 2015, 51, 3147-3150.

(282) Nguyen, H. D.; Dang, D. T.; van Dongen, J. L. J.; Brunsveld, L. Protein Dimerization Induced by Supramolecular Interactions with Cucurbit[8]uril. Angew. Chem. Int. Ed. 2010, 49, 895-898.

(283) Fang, R.; Liu, Y.; Wang, Z.; Zhang, X. Water-Soluble Supramolecular Hyperbranched Polymers Based on host-Enhanced pi-pi Interaction. Polym. Chem. 2013, 4, 900-903.

(284) Yang, H.; Ma, Z.; Wang, Z.; Zhang, X. Fabricating Covalently Attached Hyperbranched Polymers by Combining Photochemistry with Supramolecular Polymerization. Polym. Chem. 2014, 5, 1471-1476.

(285) Liu, Y.; Huang, Z.; Liu, K.; Kelgtermans, H.; Dehaen, W.; Wang, Z.; Zhang, X. Porphyrin-Containing Hyperbranched Supramolecular Polymers: Enhancing O- 
1(2)-Generation Efficiency by Supramolecular Polymerization. Polym. Chem. 2014, $5,53-56$.

(286) Zhang, L.; Zhou, T.-Y.; Tian, J.; Wang, H.; Zhang, D.-W.; Zhao, X.; Liu, Y.; Li, Z.-T. A Two-dimensional Single-Layer Supramolecular Organic Framework that is Driven by Viologen Radical Cation Dimerization and Further Promoted by Cucurbit[8]uril. Polym. Chem. 2014, 5, 4715-4721.

(287) Zhang, K. D.; Tian, J.; Hanifi, D.; Zhang, Y.; Sue, A. C. H.; Zhou, T. Y.; Zhang, L.; Zhao, X.; Liu, Y.; Li, Z. T. Toward a Single-Layer Two-Dimensional Honeycomb Supramolecular Organic Framework in Water. J. Am. Chem. Soc. 2013, 135, 1791317918.

(288) Moon, K.; Grindstaff, J.; Sobransingh, D.; Kaifer, A. E. Cucurbit[8]uril-mediated Redox-Controlled Self-Assembly of Viologen-containing Dendrimers. Angew. Chem. Int. Ed. 2004, 43, 5496-5499.

(289) Ziganshina, A. Y.; Ko, Y. H.; Jeon, W. S.; Kim, K. Stable [pi]-Dimer of a Tetrathiafulvalene Cation Radical Encapsulated in the Cavity of Cucurbit[8]uril. Chem. Commun. 2004, 806-807.

(290) Spanggaard, H.; Prehn, J.; Nielsen, M. B.; Levillain, E.; Allain, M.; Becher, J. Multiple-bridged Bis-tetrathiafulvalenes: New Synthetic Protocols and Spectroelectrochemical Investigations. J. Am. Chem. Soc. 2000, 122, 9486-9494.

(291) Coskun, A.; Spruell, J. M.; Barin, G.; Fahrenbach, A. C.; Forgan, R. S.; Colvin, M. T.; Carmieli, R.; Benitez, D.; Tkatchouk, E.; Friedman, D. C.; Sarjeant, A. A.; Wasielewski, M. R.; Goddard III, W. A.; Stoddart, J. F. Mechanically Stabilized Tetrathiafulvalene Radical Dimers. J. Am. Chem. Soc. 2011, 133, 4538-4547.

(292) Frasconi, M.; Kikuchi, T.; Cao, D.; Wu, Y.; Liu, W. G.; Dyar, S. M.; Barin, G.; Sarjeant, A. A.; Stern, C. L.; Carmieli, R.; Wang, C.; Wasielewski, M. R.; Goddard 
III, W. A.; Stoddart, J. F. Mechanical Bonds and Topological Effects in Radical Dimer Stabilization. J. Am. Chem. Soc. 2014, 136, 11011-11026.

(293) Tian, J.; Ding, Y.-D.; Zhou, T. Y.; Zhang, K. D.; Zhao, X.; Wang, H.; Zhang, D. W.; Liu, Y.; Li, Z. T. Self-Assembly of Three-Dimensional Supramolecular Polymers through Cooperative Tetrathiafulvalene Radical Cation Dimerization. Chem. Eur. J. 2014, 20, 575-584.

(294) Chen, L.; Zhang, S. C.; Wang, H.; Zhou, Y. M.; Li, Z. T.; Zhang, D. W. ThreeDimensional Supramolecular Polymers Driven by Rigid Tetrahedral Building Blocks Through Tetrathiafulvalene Radical Cation Dimerization. Tetrahedron 2014, $70,4778-4783$.

(295) Li, S. L.; Xiao, T.; Lin, C.; Wang, L. Advanced Supramolecular Polymers Constructed by Orthogonal Self-assembly. Chem. Soc. Rev. 2012, 41, 5950-5968.

(296) Wei, P.; Yan, X.; Huang, F. Supramolecular Polymers Constructed by Orthogonal Self-Assembly Based on Host-Guest and Metal-Ligand Interactions. Chem. Soc. Rev. $2015,44,815-832$.

(297) Kim, K. Mechanically Interlocked Molecules Incorporating Cucurbituril and their Supramolecular Assemblies. Chem. Soc. Rev. 2002, 31, 96-107.

(298) Robins, M. J.; Wnuk, S. F.; Hernández-Thirring, A. E.; Samano, M. C. Nucleic Acid Related Compounds. 91. Biomimetic Reactions Are in Harmony with Loss of 2'-Substituents as Free Radicals (Not Anions) during Mechanism-Based Inactivation of Ribonucleotide Reductases. Differential Interactions of Azide, Halogen, and Alkylthio. J. Am. Chem. Soc. 1996, 118, 11341-11348.

(299) Whang, D.; Kim, K. Polycatenated Two-Dimensional Polyrotaxane Net. J. Am. Chem. Soc. 1997, 119, 451-452. 
(300) Park, K. M.; Lee, E.; Roh, S. G.; Kim, J.; Kim, K. A Double-Chained Polyrotaxane: Cucurbituril ‘Beads’ Threaded onto a Double-chained One-Dimensional Coordination Polymer. Bull. Korean Chem. Soc. 2004, 25, 1711-1713.

(301) Liu, Y.; Huang, Z.; Tan, X.; Wang, Z.; Zhang, X. Cucurbit[8]uril-Based Supramolecular Polymers: Promoting Supramolecular Polymerization by Metal-Coordination. Chem. Commun. 2013, 49, 5766-5768.

(302) Joseph, R.; Nkrumah, A.; Clark, R. J.; Masson, E. Stabilization of Cucurbituril/Guest Assemblies via Long-Range Coulombic and CH-O Interactions. J. Am. Chem. Soc. 2014, 136, 6602-6607.

(303) Patrick, C. R.; Prosser, G. S. A Molecular Complex of Benzene and Hexafluorobenzene. Nature 1960, 187, 1021.

(304) Coates, G. W.; Dunn, A. R.; Henling, L. M.; Dougherty, D. A.; Grubbs, R. H. PhenylPerfluorophenyl Stacking Interactions: A New Strategy for Supermolecule Construction. Angew. Chem. Int. Ed. 1997, 36, 248-251.

(305) Yang, H.; Bai, Y.; Yu, B.; Wang, Z.; Zhang, X. Supramolecular Polymers Bearing Disulfide Bonds. Polym. Chem. 2014, 5, 6439-6443.

(306) Song, Q.; Li, F.; Tan, X.; Yang, L.; Wang, Z.; Zhang, X. Supramolecular Polymerization of Supramonomers: a way for Fabricating Supramolecular Polymers. Polym. Chem. 2014, 5, 5895-5899.

(307) Tan, X.; Yang, L.; Huang, Z.; Yu, Y.; Wang, Z.; Zhang, X. Amphiphilic DiselenideContaining Supramolecular Polymers. Polym. Chem. 2015, 6, 681-685.

(308) Hou, C.; Li, J.; Zhao, L.; Zhang, W.; Luo, Q.; Dong, Z.; Xu, J.; Liu, J. Construction of Protein Nanowires through Cucurbit[8]uril-based Highly Specific Host-Guest 
Interactions: An Approach to the Assembly of Functional Proteins. Angew. Chem. Int. Ed. 2013, 52, 5590-5593.

(309) Huang, Z.; Yang, L.; Liu, Y.; Wang, Z.; Scherman, O. A.; Zhang, X. Supramolecular Polymerization Promoted and Controlled through Self-Sorting. Angew. Chem. Int. Ed. 2014, 53, 5351-5355.

(310) Rauwald, U.; Scherman, O. A. Supramolecular Block Copolymers with Cucurbit[8]uril in Water. Angew. Chem. Int. Ed. 2008, 47, 3950-3953.

(311) Chen, C. J.; Li, D. D.; Wang, H. B.; Zhao, J.; Ji, J. Fabrication of Dual-Responsive Micelles Based on the Supramolecular Interaction of Cucurbit[8]uril. Polym. Chem. 2013, 4, 242-245.

(312) Ji, Z.; Liu, J.; Chen, G.; Jiang, M. A Polymeric Chain Extension Driven by HSCT Interaction. Polym. Chem. 2014, 5, 2709-2714.

(313) Geng, J.; Jiao, D.; Rauwald, U.; Scherman, O. A. An Aqueous Supramolecular SideChain Polymer Designed for Molecular Loading. Aust. J. Chem. 2010, 63, 627-630.

(314) Geng, J.; Biedermann, F.; Zayed, J. M.; Tian, F.; Scherman, O. A. Supramolecular Glycopolymers in Water: A Reversible Route Toward Multivalent CarbohydrateLectin Conjugates Using Cucurbit[8]uril. Macromolecules 2011, 44, 4276-4281.

(315) Loh, X. J.; del Barrio, J.; Toh, P. P. C.; Lee, T.-C.; Jiao, D.; Rauwald, U.; Appel, E. A.; Scherman, O. A. Triply Triggered Doxorubicin Release From Supramolecular Nanocontainers. Biomacromolecules 2012, 13, 84-91.

(316) Zhao, J.; Chen, C.; Li, D.; Liu, X.; Wang, H.; Jin, Q.; Ji, J. Biocompatible and Biodegradable Supramolecular Assemblies formed with Cucurbit[8]uril as a Smart Platform for Reduction-Triggered Release of Doxorubicin. Polym. Chem. 2014, 5, 1843-1847. 
(317) Jiao, D.; Geng, J.; Loh, X. J.; Das, D.; Lee, T. C.; Scherman, O. A. Supramolecular Peptide Amphiphile Vesicles through HostGuest Complexation. Angew. Chem. Int. Ed. 2012, 51, 9633-9637.

(318) Loh, X. J.; del Barrio, J.; Lee, T. C.; Scherman, O. A. Supramolecular Polymeric Peptide Amphiphile Vesicles for the Encapsulation of Basic Fibroblast Growth Factor. Chem. Commun. 2014, 50, 3033-3035.

(319) Mondal, J. H.; Ahmed, S.; Das, D. Physicochemical Analysis of Mixed Micelles of a Viologen Surfactant: Extended to Water-in-Oil (w/o) Microemulsion and Cucurbit[8] uril-Assisted Vesicle Formation. Langmuir 2014, 30, 8290-8299.

(320) Mondal, J. H.; Ghosh, T.; Ahmed, S.; Das, D. Dual Self-Sorting by Cucurbit[8]uril To Transform a Mixed Micelle to Vesicle. Langmuir 2014, 30, 11528-11534.

(321) Appel, E. A.; Biedermann, F.; Rauwald, U.; Jones, S. T.; Zayed, J. M.; Scherman, O. A. Supramolecular Cross-Linked Networks via Host-Guest Complexation with $\mathrm{Cu}$ curbit[8]uril. J. Am. Chem. Soc. 2010, 132, 14251-14260.

(322) Appel, E. A.; Loh, X. J.; Jones, S. T.; Biedermann, F.; Dreiss, C. A.; Scherman, O. A. Ultrahigh-Water-Content Supramolecular Hydrogels Exhibiting Multistimuli Responsiveness. J. Am. Chem. Soc. 2012, 134, 11767-73.

(323) Appel, E. A.; Loh, X. J.; Jones, S. T.; Dreiss, C. A.; Scherman, O. A. Sustained Release of Proteins from High Water Content Supramolecular Polymer Hydrogels. Biomaterials 2012, 33, 4646-4652.

(324) Walsh, Z.; Janeček, E.-R.; Hodgkinson, J. T.; Sedlmair, J.; Koutsioubas, A.; Spring, D. R.; Welch, M.; Hirschmugl, C. J.; Toprakcioglu, C.; Nitschke, J. R.; Jones, M.; Scherman, O. A. Multifunctional Supramolecular Polymer Networks as Next-generation Consolidants for Archaeological wood Conservation. Proc. Natl. Acad. Sci USA 2014, 111, 17743-17748. 
(325) Rowland, M. J.; Appel, E. A.; Coulston, R. J.; Scherman, O. A. Dynamically Crosslinked Materials via Recognition of Amino Acids by Cucurbit[8]uril. J. Mater. Chem. B 2013, 1, 2904-2910.

(326) Appel, E. A.; Dyson, J.; del Barrio, J.; Walsh, Z.; Scherman, O. A. Formation of Single-Chain Polymer Nanoparticles in Water through Host-Guest Interactions. Angew. Chem. Int. Ed. 2012, 51, 4185-4189.

(327) Lin, Y.; Li, L.; Li, G. A New Supramolecular Gel via Host-Guest Complexation with Cucurbit[8]uril and N-(4-diethylaminobenzyl)chitosan. Carbohyd. Polym. 2013, 92, 429-434.

(328) Appel, E. A.; Forster, R. A.; Koutsioubas, A.; Toprakcioglu, C.; Scherman, O. A. Activation Energies Control the Macroscopic Properties of Physically Cross-Linked Materials. Angew. Chem. Int. Ed. 2014, 53, 10038-10043.

(329) Appel, E. A.; Forster, R. A.; Rowland, M. J.; Scherman, O. A. The Control of Cargo Release from Physically Crosslinked Hydrogels by Crosslink Dynamics. Biomaterials 2014, 35, 9897-9903.

(330) McKee, J. R.; Appel, E. A.; Seitsonen, J.; Kontturi, E.; Scherman, O. A.; Ikkala, O. Healable, Stable and Stiff Hydrogels: Combining Conflicting Properties Using Dynamic and Selective Three-Component Recognition with Reinforcing Cellulose Nanorods. Adv. Funct. Mater. 2014, 24, 2706-2713.

(331) Janeček, E.-R.; McKee, J. R.; Tan, C. S. Y.; Nykänen, A.; Kettunen, M.; Laine, J.; Ikkala, O.; Scherman, O. A. Hybrid Supramolecular and Colloidal Hydrogels that Bridge Multiple Length Scales. Angew. Chem. Int. Ed. 2015, 54, 5383-5388.

(332) Li, C.; Rowland, M. J.; Shao, Y.; Cao, T.; Chen, C.; Jia, H.; Zhou, X.; Yang, Z.; Scherman, O. A.; Liu, D. Responsive Double Network Hydrogels of Interpenetrating DNA and CB[8] Host-Guest Supramolecular Systems. Adv. Mater. 2015, 3298-3304. 
(333) Park, K. M.; Yang, J. A.; Jung, H.; Yeom, J.; Park, J. S.; Park, K. H.; Hoffman, A. S.; Hahn, S. K.; Kim, K. In Situ Supramolecular Assembly and Modular Modification of Hyaluronic Acid Hydrogels for 3D Cellular Engineering. ACS Nano 2012, 6, 29602968.

(334) Jung, H.; Park, J. S.; Yeom, J.; Selvapalam, N.; Park, K. M.; Oh, K.; Yang, J. A.; Park, K. H.; Hahn, S. K.; Kim, K. 3D Tissue Engineered Supramolecular Hydrogels for Controlled Chondrogenesis of Human Mesenchymal Stem Cells. Biomacromolecules 2014, 15, 707-714.

(335) Coulston, R. J.; Jones, S. T.; Lee, T.-C.; Appel, E. A.; Scherman, O. A. Supramolecular Gold Nanoparticle-Polymer Composites formed in Water. Chem. Commun. 2011, 47, 164-166.

(336) Zhang, J.; Coulston, R. J.; Jones, S. T.; Geng, J.; Scherman, O. A.; Abell, C. One-Step Fabrication of Supramolecular Microcapsules from Microfluidic Droplets. Science 2012, 335, 690-694.

(337) Stephenson, G.; Parker, R. M.; Lan, Y.; Yu, Z.; Scherman, O. A.; Abell, C. Supramolecular Colloidosomes: Fabrication, Characterisation and Triggered Release of Cargo. Chem. Commun. 2014, 50, 7048-7051.

(338) Zheng, Y.; Yu, Z.; Parker, R. M.; Wu, Y.; Abell, C.; Scherman, O. A. Interfacial Assembly of Dendritic Microcapsules with Host-guest Chemistry. Nat. Commun. 2014, 5.

(339) Mock, W. L.; Irra, T. a.; Wepsiec, J. P.; Adhya, M. Catalysis by Cucurbituril. The Significance of Bound-Substrate Destabilization for Induced Triazole Formation. J. Org. Chem. 1989, 54, 5302-5308.

(340) Klöck, C.; Dsouza, R. N.; Nau, W. M. Cucurbituril-Mediated Supramolecular. Org. Letters 2009, 11, 2595-2598. 
(341) Hang, C.; Fang-fang, Z.; Jian-xin, Z.; Xi, Z.; Zhu, T.; Sai-feng, X.; Qian-jiang, Z. Rapid Transformation of Benzylic Alcohols to Aldehyde in the Presence of Cucurbit[8]uril. Catal. Commun. 2009, 11, 167-170.

(342) Lu, X.; Masson, E. Silver-Promoted Desilylation Catalyzed by Ortho- and Allosteric Cucurbiturils. Org. Letters 2010, 12, 2310-2313.

(343) Jon, S. Y.; Ko, Y. H.; Park, S. H.; Kim, H. J.; Kim, K. A Facile, Stereoselective [2 + 2] Photoreaction Mediated by Cucurbit[8]uril. Chem. Commun. 2001, 1938-1939.

(344) Berbeci, L. S.; Wang, W.; Kaifer, A. E. Drastically Decreased Reactivity of Thiols and Disulfides Complexed by Cucurbit[6]uril. Org. Letters 2008, 10, 3721-3724.

(345) Tuncel, D.; Steinke, J. H. G. Catalytically Self-Threading Polyrotaxanes. Chem. Commun. 1999, 1509-1510.

(346) Tuncel, D.; Steinke, J. H. G. Mainchain Pseudopolyrotaxanes via Post-Threading with Cucurbituril. Chem. Commun. 2001, 253-254.

(347) Tuncel, D.; Steinke, J. H. G. The Synthesis of [2], [3] and [4] Rotaxanes and Semirotaxanes. Chem. Commun. 2002, 496-497.

(348) Tuncel, D.; Katterle, M. PH-Triggered Dethreading-Rethreading and Switching of Cucurbit[6]uril on Bistable [3]pseudorotaxanes and [3]rotaxanes. Chem. Eur. J. 2008, $14,4110-4116$.

(349) Tuncel, D.; Ünal, O.; Artar, M. Supramolecular Assemblies Constructed by Cucurbituril-Catalyzed Click Reaction. Isr. J. Chem. 2011, 51, 525-532.

(350) Basilio, N.; García-Río, L.; Moreira, J. A.; Pessêgo, M. Supramolecular Catalysis by Cucurbit[7]uril and Cyclodextrins: Similarity and Differences. J. Org. Chem 2010, 75, 848-855. 
(351) Wang, Y. H.; Cong, H.; Zhao, F. F.; Xue, S. F.; Tao, Z.; Zhu, Q. J.; Wei, G. Selective Catalysis for the Oxidation of Alcohols to Aldehydes in the Presence of Cucurbit[8]uril. Catal. Commun. 2011, 12, 1127-1130.

(352) Pattabiraman, M.; Natarajan, A.; Kaliappan, R.; Mague, J. T.; Ramamurthy, V. Template Directed Photodimerization of Trans-1\{,\}2-bis(n-pyridyl)ethylenes and Stilbazoles in Water. Chem. Commun. 2005, 4542-4544.

(353) Maddipatla, M. V. S. N.; Kaanumalle, L. S.; Natarajan, A.; Pattabiraman, M.; Ramamurthy, V. Preorientation of Olefins toward a Single Photodimer: CucurbiturilMediated Photodimerization of Protonated Azastilbenes in Water. Langmuir 2007, $23,7545-7554$.

(354) Pemberton, B. C.; Barooah, N.; Srivatsava, D. K.; Sivaguru, J. Supramolecular Photocatalysis by Confinement-Photodimerization of Coumarins within Cucurbit[8]urils. Chem. Commun. 2010, 46, 225-227.

(355) Pattabiraman, M.; Kaanumalle, L. S.; Natarajan, A.; Ramamurthy, V. Regioselective Photodimerization of Cinnamic Acids in Water: Templation with Cucurbiturils. Langmuir 2006, 22, 7605-7609.

(356) Chen, B.; Cheng, S. F.; Liao, G. H.; Li, X.-W.; Zhang, L. P.; Tung, C. H.; Wu, L. Z. Efficient and Selective Photodimerization of 2-Naphthalenecarbonitrile Mediated by Cucurbit[8]uril in an Aqueous Solution. Photochem. Photobiol. Sci. 2011, 10, 14411444 .

(357) Smitka, J.; Lemos, A.; Porel, M.; Jockusch, S.; Belderrain, T. R.; Tesaová, E.; Da Silva, J. P. Phototransformation of Benzimidazole and Thiabendazole inside Cucurbit[8]uril. Photochem. Photobiol. Sci. 2014, 13, 310-5.

(358) De Lima, S. M.; Gómez, J. A.; Barros, V. P.; Vertuan, G. D. S.; Assis, M. D. D.; Graeff, C. F. D. O.; Demets, G. J. F. A New Oxovanadium(IV)-Cucurbit[6]uril Com- 
plex: Properties and Potential for Confined Heterogeneous Catalytic Oxidation Reactions. Polyhedron 2010, 29, 3008-3013.

(359) Koner, A. L.; Márquez, C.; Dickman, M. H.; Nau, W. M. Transition-Metal-Promoted Chemoselective Photoreactions at the Cucurbituril Rim. Angew. Chem. Int. Ed. 2011, $50,545-548$.

(360) Masson, E.; Shaker, Y. M.; Masson, J. P.; Kordesch, M. E.; Yuwono, C. "Supramolecular Circuitry": Three Chemiluminescent, Cucurbit[7]uril-Controlled On/Off Switches. Org. Letters 2011, 13, 3872-3875.

(361) Miskolczy, Z.; Megyesi, M.; Tárkányi, G.; Mizsei, R.; Biczók, L. Inclusion Complex Formation of Sanguinarine Alkaloid with Cucurbit[7]uril: Inhibition of Nucleophilic Attack and Photooxidation. Organic \& biomolecular chemistry 2011, 9, 10611070.

(362) Cong, H.; Li, C.-R.; Xue, S.-F.; Tao, Z.; Zhu, Q.-J.; Wei, G. Cucurbituril-Resisted Acylation of the Anti-Tuberculosis Drug Isoniazid via a Supramolecular Strategy. Org. Biomol. Chem. 2011, 9, 1041-1046.

(363) Ghosh, S.; Isaacs, L. Biological Catalysis Regulated by Cucurbit[7]uril Molecular Containers. J. Am. Chem. Soc. 2010, 132, 4445-4454.

(364) Buschmann, H. J.; Mutihac, L.; Mutihac, R. C.; Schollmeyer, E. Complexation Behavior of Cucurbit[6]uril with Short Polypeptides. Thermochim. Acta 2005, 430, 7982.

(365) Reczek, J. J.; Kennedy, A. A.; Halbert, B. T.; Urbach, A. R. Multivalent Recognition of Peptides by Modular Self-Assembled Receptors. J. Am. Chem. Soc. 2009, 131, 24082415. 
(366) Logsdon, L. A.; Schardon, C. L.; Ramalingam, V.; Kwee, S. K.; Urbach, A. R. Nanomolar Binding of Peptides Containing Noncanonical Amino Acids by a Synthetic Receptor. J. Am. Chem. Soc. 2011, 133, 17087-17092.

(367) Logsdon, L. A.; Urbach, A. R. Sequence-Specific Inhibition of a Nonspecific Protease. J. Am. Chem. Soc. 2013, 135, 11414-11416.

(368) Ghale, G.; Ramalingam, V.; Urbach, A. R.; Nau, W. M. Determining Protease Substrate Selectivity and Inhibition by Label-Free Supramolecular Tandem Enzyme Assays. J. Am. Chem. Soc. 2011, 133, 7528-7535.

(369) Dang, D. T.; Bosmans, R. P. G.; Moitzi, C.; Voets, I. K.; Brunsveld, L. Solution Structure of a Cucurbit[8]uril Induced Compact Supramolecular Protein Dimer. Org. Biomol. Chem. 2014, 12, 9341-9344.

(370) Dang, D. T.; Schill, J.; Brunsveld, L. Cucurbit[8]uril-Mediated Protein Homotetramerization. Chem. Sci. 2012, 3, 2679-2684.

(371) Dang, D. T.; Nguyen, H. D.; Merkx, M.; Brunsveld, L. Supramolecular Control of Enzyme Activity through Cucurbit[8]uril-Mediated Dimerization. Angewandte Chemie 2013, 125, 2987-2991.

(372) Lee, H. H.; Choi, T. S.; Lee, S. J. C.; Lee, J. W.; Park, J.; Ko, Y. H.; Kim, W. J.; Kim, K.; Kim, H. I. Supramolecular Inhibition of Amyloid Fibrillation by Cucurbit[7]uril. Angewandte Chemie 2014, 126, 7591-7595.

(373) Heo, S. W.; Choi, T. S.; Park, K. M.; Ko, Y. H.; Kim, S. B.; Kim, K.; Kim, H. I. HostGuest Chemistry in the Gas Phase: Selected Fragmentations of CB[6]-Peptide Complexes at Lysine Residues and Its Utility to Probe the Structures of Small Proteins. Anal. Chem. 2011, 83, 7916-7923. 
(374) Lee, J. W.; Heo, S. W.; Lee, S. J. C.; Ko, J. Y.; Kim, H. I. H.; Kim, H. I. H. Probing Conformational Changes of Ubiquitin by Host-Guest Chemistry using Electrospray Ionization Mass Spectrometry. J. Am. Soc. Mass Spectrom. 2013, 24, 21-29.

(375) Parente Carvalho, C.; Norouzy, A.; Ribeiro, V.; Nau, W. M.; Pischel, U. Cucurbiturils as Supramolecular Inhibitors of DNA Restriction by Type II Endonucleases. Org. Biomol. Chem. 2015, 2866-2869.

(376) Rekharsky, M. V.; Mori, T.; Yang, C.; Ko, Y. H.; Selvapalam, N.; Kim, H.; Sobransingh, D.; Kaifer, A. E.; Liu, S.; Isaacs, L.; Chen, W.; Moghaddam, S.; Gilson, M. K.; Kim, K.; Inoue, Y. A Synthetic Host-Guest System Achieves Avidin-Biotin Affinity by Overcoming EnthalpyEntropy Compensation. Proc. Natl. Acad. Sci USA 2007, $104,20737-20742$.

(377) Mock, W. L.; Shih, N. Y. Structure and Selectivity in Host-Guest Complexes of Cucurbituril. J. Org. Chem 1986, 51, 4440-4446.

(378) Zhang, H.; Ferrell, T. A.; Asplund, M. C.; Dearden, D. V. Molecular Beads on a Charged Molecular String: Alpha,Omega-Alkyldiammonium Complexes of Cucurbit[6]uril in the Gas Phase. Int. J. Mass Spectrom. 2007, 265, 187-196.

(379) Rekharsky, M. V.; Yamamura, H.; Mori, T.; Sato, A.; Shiro, M.; Lindeman, S. V.; Rathore, R.; Shiba, K.; Ko, Y. H.; Selvapalam, N.; Kim, K.; Inoue, Y. Supramolecular Complexation of N-alkyl- and $\mathrm{N}, \mathrm{N}^{\prime}$-Dialkylpiperazines with Cucurbit[6]uril in Aqueous Solution and in the Solid State. Chemistry 2009, 15, 1957-1965.

(380) Occello, V. N. S.; Rossi, R. H. D.; Veglia, A. V.; Sueldo Occello, V. N.; de Rossi, R. H.; Veglia, A. V. Complexation (Cucurbit[6]uril-Pyrene): Thermodynamic and Spectroscopic Properties. J. Lumin. 2015, 158, 435-440.

(381) Choi, T. S.; Ko, J. Y.; Heo, S. W.; Ko, Y. H.; Kim, K.; Kim, H. I. Unusual Complex 
Formation and Chemical Reaction of Haloacetate Anion on the Exterior Surface of Sucurbit[6]uril in the Gas Phase. J. Am. Soc. Mass Spectrom. 2012, 23, 1786-1793.

(382) Ong, W.; Kaifer, A. E. Unusual Electrochemical Properties of the Inclusion Complexes of Ferrocenium and Cobaltocenium with Cucurbit[7]uril. Organometallics 2003, 22, 4181-4183.

(383) Jeon, W. S.; Moon, K.; Park, S. H.; Chun, H.; Ko, Y. H.; Lee, J. Y.; Lee, E. S.; Samal, S.; Selvapalam, N.; Rekharsky, M. V.; Sindelar, V.; Sobransingh, D.; Inoue, Y.; Kaifer, A. E.; Kim, K. Complexation of Ferrocene Derivatives by the Cucurbit[7]uril Host: A Comparative Study of the Cucurbituril and Cyclodextrin Host Families. J. Am. Chem. Soc. 2005, 127, 12984-12989.

(384) Moghaddam, S.; Yang, C.; Rekharsky, M.; Ko, Y. H.; Kim, K.; Inoue, Y.; Gilson, M. K. New Ultrahigh Affinity Host-Guest Complexes of Cucurbit[7]uril with Bicyclo[2.2.2]octane and Adamantane Guests: Thermodynamic Analysis and Evaluation of M2 Affinity Calculations. J. Am. Chem. Soc. 2011, 133, 3570-3581.

(385) Cao, L.; Šekutor, M.; Zavalij, P. Y.; Mlinarić-Majerski, K.; Glaser, R.; Isaacs, L. Cucurbit[7]urilGuest Pair with an Attomolar Dissociation Constant. Angew. Chem. Int. Ed. 2014, 53, 988-993.

(386) Wang, R.; Yuan, L.; Macartney, D. H. Stabilization of the (E)-1-Ferrocenyl2-(1-methyl-4-pyridinium)ethylene Cation by Inclusion in Cucurbit[7]uril. Organometallics 2006, 25, 1820-1823.

(387) Ma, D.; Zavalij, P. Y.; Isaacs, L. Acyclic Cucurbit[n]uril Congeners Are High Affinity Hosts. J. Org. Chem 2010, 75, 4786-4795.

(388) Ong, W.; Kaifer, A. E. Salt Effects on the Apparent Stability of the Cucurbit[7]urilMethyl Viologen Inclusion Complex. J. Org. Chem 2004, 69, 1383-1385. 
(389) Ling, Y.; Mague, J. T.; Kaifer, A. E. Inclusion Complexation of Diquat and Paraquat by the Hosts Cucurbit[7]uril and Cucurbit[8]uril. Chem.-Eur. J. 2007, 13, 7908-7914.

(390) Sindelar, V.; Cejas, M. A.; Raymo, F. M.; Kaifer, A. E. Tight Inclusion Complexation of 2,7-Dimethyldiazapyrenium in Cucurbit[7]uril. New J. Chem. 2005, 29, 280-282.

(391) Wintgens, V.; Biczók, L.; Miskolczy, Z. Thermodynamics of Inclusion Complex Formation Between 1-alkyl-3-methylimidazolium Ionic Liquids and Cucurbit[7]uril. Supramol. Chem. 2010, 22, 612-618.

(392) Miskolczy, Z.; Biczók, L.; Megyesi, M.; Jablonkai, I. Inclusion Complex Formation of Ionic Liquids and Other Cationic Organic Compounds with Cucurbit[7]uril Studied by 4',6-Diamidino-2-phenylindole Fluorescent Probe. J. Phys. Chem. B 2009, 113, $1645-1651$.

(393) Megyesi, M.; Biczók, L.; Jablonkai, I. Highly Sensitive Fluorescence Response to Inclusion Complex Formation of Berberine Alkaloid with Cucurbit[7]uril. J. Phys. Chem. C 2008, 112, 3410-3416.

(394) Shaikh, M.; Mohanty, J.; Bhasikuttan, A. C.; Uzunova, V. D.; Nau, W. M.; Pal, H. SaltInduced Guest Relocation from a Macrocyclic Cavity into a Biomolecular Pocket: Interplay between Cucurbit[7]uril and Albumin. Chem. Commun. 2008, 3681-3683.

(395) Liu, Y.; Li, C.-J.; Guo, D.-S.; Pan, Z.-H.; Li, Z. A Comparative Study of Complexation of $\beta$-Cyclodextrin, Calix[4]arenesulfonate and Cucurbit[7]uril with Dye Guests: Fluorescence Behavior and Binding Ability. Supramol. Chem. 2007, 19, 517523.

(396) Choudhury, S. D.; Mohanty, J.; Pal, H.; Bhasikuttan, A. C. Cooperative Metal Ion Binding to a Cucurbit[7]urilThioflavin T Complex: Demonstration of a StimulusResponsive Fluorescent Supramolecular Capsule. J. Am. Chem. Soc. 2010, 132, 13951401. 
(397) Mohanty, J.; Bhasikuttan, A. C.; Choudhury, S. D.; Pal, H. Noncovalent Interaction of 5,10,15,20-Tetrakis(4-N-methylpyridyl)porphyrin with Cucurbit[7]uril: A Supramolecular Architecture. J. Phys. Chem. B 2008, 112, 10782-10785.

(398) St-Jacques, A. D.; Wyman, I. W.; Macartney, D. H. Encapsulation of Charge-Diffuse Peralkylated Onium Cations in the Cavity of Cucurbit[7]uril. Chem. Commun. 2008, 4936-4938.

(399) Freitag, M.; Gundlach, L.; Piotrowiak, P.; Galoppini, E. Fluorescence Enhancement of Di-p-tolyl Viologen by Complexation in Cucurbit[7]uril. J. Am. Chem. Soc. 2012, $134,3358-3366$.

(400) Sindelar, V.; Parker, S. E.; Kaifer, A. E. Inclusion of Anthraquinone Derivatives by the Cucurbit[7]uril Host. New J. Chem. 2007, 31, 725-728.

(401) Chakraborty, B.; Basu, S. Deciphering the Host-Guest chemistry of Acridine Yellow and Cucurbit[7]uril: An Integrated Spectroscopic and Calorimetric Study. Chem. Phys. Lett. 2011, 507, 74-79.

(402) Zhou, Y.; Yu, H.; Zhang, L.; Sun, J.; Wu, L.; Lu, Q.; Wang, L. Host Properties of Cucurbit [7] uril: Fluorescence Enhancement of Acridine Orange. J. Inc. Phenom. Macro. 2008, 61, 259-264.

(403) Montes-Navajas, P.; Corma, A.; Garcia, H. Complexation and Fluorescence of Tricyclic Basic Dyes Encapsulated in Cucurbiturils. Chem. Phys. Chem. 2008, 9, 713-720.

(404) Wang, R.; Wyman, I. W.; Wang, S.; Macartney, D. H. Encapsulation of a Betacarboline in Cucurbit[7]uril. J. Inc. Phenom. Macro. 2009, 64, 233-237.

(405) Cao, L.; Isaacs, L. Absolute and Relative Binding Affinity of Cucurbit[7]uril Towards a Series of Cationic Guests. Supramol. Chem. 2013, 26, 251-258. 
(406) Miskolczy, Z.; Harangozo, J. G.; Biczok, L.; Wintgens, V.; Lorthioir, C.; Amiel, C. Effect of Torsional Isomerization and Inclusion Complex Formation with Cucurbit[7]uril on the Fluorescence of 6-methoxy-1-methylquinolinium. Photochem. Photobiol. Sci. 2014, 13, 499-508.

(407) Lee, J. W.; Lee, H. H. L.; Ko, Y. H.; Kim, K.; Kim, H. I. Deciphering the Specific High-Affinity Binding of Cucurbit[7]uril to Amino Acids in Water. J. Phys. Chem. B $2015,119,4628-4636$.

(408) Hang, C.; Ling, T. L.; Yi-Hua, Y.; Fan, Y.; Ying, D.; Sai-Feng, X.; Zhu, T. Molecular Recognition of Aminoacid by Cucurbiturils. 2006.

(409) Bailey, D. M.; Hennig, A.; Uzunova, V. D.; Nau, W. M. Supramolecular Tandem Enzyme Assays for Multiparameter Sensor Arrays and Enantiomeric Excess Determination of Amino Acids. Chem.-Eur. J. 2008, 14, 6069-6077.

(410) Wyman, I. W.; Macartney, D. H. Host-guest Complexations of Local Anaesthetics by Cucurbit[7]uril in Aqueous Solution. Org. Biomol. Chem. 2010, 8, 247-252.

(411) Wyman, I. W.; Macartney, D. H. Cucurbit[7]uril Host-Guest Complexes of Cholines and Phosphonium Cholines in Aqueous Solution. Org. Biomol. Chem. 2010, 8, 253260.

(412) Wang, R.; Macartney, D. H. Cucurbit[7]uril Stabilization of a Diarylmethane Carbocation in Aqueous Solution. Tetrahedron Lett. 2008, 49, 311-314.

(413) Hettiarachchi, D. S. N.; Macartney, D. H. Cucurbit[7]uril Host-Guest Complexes with Cationic Bis(4,5-dihydro-1H-imidazol-2-yl) Guests in Aqueous Solution. Can. J. Chem. 2006, 84, 905-914.

(414) Choi, S.; Park, S. H.; Ziganshina, A. Y.; Ko, Y. H.; Lee, J. W.; Kim, K. A Stable Cis- 
Stilbene Derivative Encapsulated in Cucurbit[7]uril. Chem. Commun. 2003, 21762177.

(415) Kuz'mina, L. G.; Vedernikov, A. I.; Lobova, N. A.; Howard, J. A. K.; Strelenko, Y. A.; Fedin, V. P.; Alfimov, M. V.; Gromov, S. P. Photoinduced and Dark Complexation of Unsaturated Viologen Analogues Containing Two Ammonium Tails with Cucurbit[8]uril. New J. Chem. 2006, 30, 458-466.

(416) Biedermann, F.; Nau, W. M. Noncovalent Chirality Sensing Ensembles for the Detection and Reaction Monitoring of Amino Acids, Peptides, Proteins, and Aromatic Drugs. Angew. Chem. Int. Ed. 2014, 53, 5694-5699. 


\section{Graphical TOC Entry}

\begin{tabular}{|c|c|}
\hline 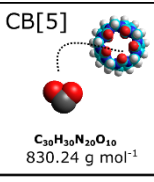 & 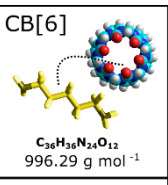 \\
\hline 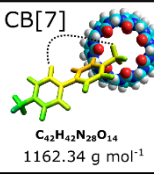 & 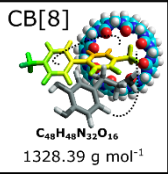 \\
\hline
\end{tabular}

